\title{
The Roots of Verbal Meaning
}




\section{OXFORD STUDIES IN THEORETICAL LINGUISTICS}

\section{General editors}

David Adger and Hagit Borer, Queen Mary University of London

\section{Advisory editors}

Stephen Anderson, Yale University; Daniel Büring, University of Vienna; Nomi Erteschik-Shir, Ben-Gurion University; Donka Farkas, University of California, Santa Cruz; Angelika Kratzer, University of Massachusetts, Amherst; Andrew Nevins, University College London; Christopher Potts, Stanford University; Barry Schein, University of Southern California; Peter Svenonius, University of Tromsø; Moira Yip, University College London

RECENT TITLES IN THE SERIES

60 Sentence and Discourse

edited by Jacqueline Guéron

61 Optimality-Theoretic Syntax, Semantics, and Pragmatics

From Uni- to Bidirectional Optimization

edited by Géraldine Legendre, Michael T. Putnam, Henriëtte de Swart, and Erin Zaroukian

62 The Morphosyntax of Transitions

A Case Study in Latin and Other Languages

by Víctor Acedo-Matellán

63 Modality Across Syntactic Categories

edited by Ana Arregui, María Luisa Rivero, and Andrés Salanova

64 The Verbal Domain

edited by Roberta D’Alessandro, Irene Franco, and Ángel J. Gallego

65 Concealed Questions

by Ilaria Frana

66 Parts of a Whole

Distributivity as a Bridge between Aspect and Measurement

by Lucas Champollion

67 Semantics and Morphosyntactic Variation

Qualities and the Grammar of Property Concepts

by Itamar Francez and Andrew Koontz-Garboden

68 The Structure of Words at the Interfaces

edited by Heather Newell, Máire Noonan, Glyne Piggott, and Lisa deMena Travis

69 Pragmatic Aspects of Scalar Modifiers

The Semantics-Pragmatics Interface

by Osamu Sawada

70 Encoding Events

Functional Structure and Variation

by Xuhui $\mathrm{Hu}$

71 Gender and Noun Classification

edited by Éric Mathieu, Myriam Dali, and Gita Zareikar

72 The Grammar of Expressivity

by Daniel Gutzmann

73 The Grammar of Copulas Across Language

edited by María J. Arche, Antonio Fábregas, and Rafael Marín

74 The Roots of Verbal Meaning

by John Beavers and Andrew Koontz-Garboden

For a complete list of titles published and in preparation for the series, see pp. 256-8. 


\title{
The Roots of Verbal Meaning
}

\author{
JOHN BEAVERS AND \\ ANDREW KOONTZ-GARBODEN
}




\section{OXFORD \\ UNIVERSITY PRESS}

\section{Great Clarendon Street, Oxford, OX2 6DP, United Kingdom}

Oxford University Press is a department of the University of Oxford. It furthers the University's objective of excellence in research, scholarship, and education by publishing worldwide. Oxford is a registered trade mark of

Oxford University Press in the UK and in certain other countries

(C) John Beavers and Andrew Koontz-Garboden 2020

The moral rights of the authors have been asserted

First Edition published in 2020

Impression: 1

Some rights reserved. No part of this publication may be reproduced, stored in a retrieval system, or transmitted, in any form or by any means, for commercial purposes, without the prior permission in writing of Oxford University Press, or as expressly

permitted by law, by licence or under terms agreed with the appropriate reprographics rights organization.

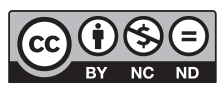

This is an open access publication, available online and distributed under the terms of a

Creative Commons Attribution - Non Commercial - No Derivatives 4.0

International licence (CC BY-NC-ND 4.0), a copy of which is available at http://creativecommons.org/licenses/by-nc-nd/4.0/.

Enquiries concerning reproduction outside the scope of this licence should be sent to the Rights Department, Oxford University Press, at the address above

Published in the United States of America by Oxford University Press 198 Madison Avenue, New York, NY 10016, United States of America

$$
\text { British Library Cataloguing in Publication Data }
$$

Data available

Library of Congress Control Number: 2019953284

ISBN 978-0-19-885578-1

Printed and bound in Great Britain by Clays Ltd, Elcograf S.p.A.

Links to third party websites are provided by Oxford in good faith and for information only. Oxford disclaims any responsibility for the materials contained in any third party website referenced in this work. 


\section{Contents}

General preface $\quad$ ix

Acknowledgments $\quad$ xi

List of abbreviations $\quad \mathrm{xv}$

Glossing conventions xvii

1. Introduction 1

1.1 Lexical semantics and theories of verb meaning 1

$\begin{array}{ll}1.2 \text { Thematic roles as a theory of verb meaning } & 6\end{array}$

$\begin{array}{ll}1.3 \text { Event structures as a theory of verb meaning } & 8\end{array}$

1.3.1 Roots, templates, and decompositional structure $\quad 8$

$\begin{array}{ll}\text { 1.3.2 A semantics for event structures } & 13\end{array}$

1.3.3 Summary: The core properties of event structures 22

$\begin{array}{ll}1.4 \text { Hypotheses about root meaning } & 23\end{array}$

1.4.1 The Bifuraction Thesis of Roots $\quad 26$

1.4.2 Manner/Result Complementarity $\quad 27$

1.4.3 The true distinction between roots and templates 28

$\begin{array}{ll}\text { 1.4.4 Comparison to prior works } & 31\end{array}$

$\begin{array}{ll}1.5 \text { Outline of the study } & 33\end{array}$

1.6 Background assumptions: Defining causation and change $\quad 35$

$\begin{array}{ll}\text { 1.6.1 Change of state as scalar change } & 35\end{array}$

1.6.2 Causation and its role in verb meaning $\quad 48$

2. Entailments of change in the roots of change-of-state verbs 57

$\begin{array}{ll}2.1 \text { Two types of change-of-state verbs } & 57\end{array}$

2.2 Lexical semantic consequences of Bifurcation 60

2.2.1 Three semantic predictions $\quad 62$

2.2.2 Entailments of change in derived statives $\quad 67$

$\begin{array}{ll}2.2 .3 \text { Sources of variation } & 71\end{array}$

2.2.4 Summary: Predictions of Bifurcation and semantic explananda $\quad 73$

$\begin{array}{ll}2.3 \text { Morphological consequences of Bifurcation } & 74\end{array}$

$\begin{array}{ll}\text { 2.3.1 Four morphological predictions } & 74\end{array}$

2.3.2 Adjectives derived from the different root classes across languages $\quad 76$

2.3.3 Summary: Predictions of Bifurcation and

$\begin{array}{ll}\text { morphological explananda } & 80\end{array}$

2.4 Analytical option 1: Abandon Bifurcation $\quad 80$

2.4.1 A purely non-lexicalist analysis $\quad 81$

$\begin{array}{ll}2.4 .2 \text { A purely lexicalist analysis } & 90\end{array}$

2.4.3 A mixed analysis $\quad 94$

$\begin{array}{ll}2.4 .4 \text { Summary } & 95\end{array}$ 
2.5 Analytical option 2: Preserving Bifurcation 96

2.5.1 Reanalyzing result roots as manner entailing 96

2.5.2 Allosemy 98

2.5.3 Root selection for syntactic construction $\quad 102$

$\begin{array}{ll}\text { 2.5.4 Summary } & 103\end{array}$

2.6 Roots vs. templates in change-of-state verbs $\quad 104$

$\begin{array}{ll}2.7 \text { Concluding remarks } & 104\end{array}$

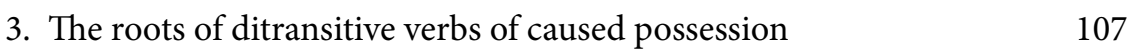

$\begin{array}{ll}3.1 \text { Roots and templates in ditransitive verbs } & 107\end{array}$

3.2 Ditransitive verbs, possession, and co-location 111

3.3 The meanings of the templates: A case for root sensitivity 114

3.3.1 The semantics of indirect object templates $\quad 115$

3.3.2 The semantics of to templates $\quad 117$

$\begin{array}{ll}\text { 3.3.3 Summary } & 120\end{array}$

3.4 Change of state and aspectual properties: Root telicity 122

3.5 Defining and composing template and root meanings 124

$\begin{array}{ll}3.6 \text { Root classes } & 130\end{array}$

$\begin{array}{ll}3.6 .1 \text { True possession roots } & 130\end{array}$

$\begin{array}{ll}3.6 .2 \text { Release roots } & 135\end{array}$

3.6.3 Arrival roots 138

3.6.4 Electronic release and arrival roots $\quad 139$

$\begin{array}{ll}3.6 .5 \text { Future having roots } & 140\end{array}$

$\begin{array}{ll}\text { 3.6.6 Summary } & 141\end{array}$

3.7 Further evidence for change of state in roots: Root durativity 142

3.8 The Dative Alternation: Root-determined argument realization $\quad 148$

3.9 Alternative analyses: Allosemy and allomorphy 150

3.10 Root vs. template in ditransitive verbs $\quad 155$

$\begin{array}{ll}3.11 \text { Concluding remarks } & 157\end{array}$

4. Manner/Result Complementarity and causation in verbal roots 159

4.1 Manner/Result Complementarity 159

4.2 Result entailments in verbs $\quad 165$

4.2.1 Result diagnostic \# 1: Denial of result 166

4.2.2 Result diagnostic \# 2: Object deletion 168

4.2.3 Result diagnostic \# 3: Restricted resultatives 169

4.3 Manner entailments in verbs $\quad 172$

4.3.1 Manner diagnostic \# 1: Selectional restrictions 173

4.3.2 Manner diagnostic \# 2: Denial of action $\quad 175$

4.3.3 Manner diagnostic \# 3: Complexity of action 177

$\begin{array}{lr}4.4 \text { Classes of manner+result verbs } & 178\end{array}$

$\begin{array}{ll}\text { 4.4.1 Manner of killing verbs } & 179\end{array}$

4.4.2 Manner of cooking verbs $\quad 184$

$\begin{array}{ll}\text { 4.4.3 Ditransitive ballistic motion verbs } & 187\end{array}$

4.4.4 Summary of manner+result verbs $\quad 189$ 
4.5 Manner, result, and the architecture of event structure 191

4.5.1 Complementarity in event structures 192

4.5.2 The decomposition of transitive manner+result predicates 195

4.5.3 Roots of transitive manner+result verbs as complements of $v \quad 200$

4.5.4 Roots of ditransitive manner+result verbs as adjuncts of $v \quad 203$

4.5.5 A typology of the roots of manner+result verbs 205

$\begin{array}{ll}\text { 4.6 The roots of manner+result verbs and Bifurcation } 206 & 205\end{array}$

4.7 Roots vs. templates in manner+result verbs 209

$\begin{array}{ll}4.8 \text { Concluding remarks } & 210\end{array}$

$\begin{array}{ll}\text { 5. Conclusion } & 213\end{array}$

5.1 Summary on conditions on root meaning 213

5.2 The origins of complex root meanings 214

$\begin{array}{ll}\text { 5.2.1 Networks of lexical entailments } & 216\end{array}$

5.2.2 Lexicalized conventional associations 222

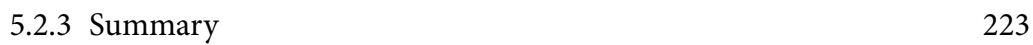

5.3 Are there any constraints on root meaning? 224

$\begin{array}{ll}5.4 \text { Possible and impossible verbs } & 226\end{array}$

$\begin{array}{ll}\text { 5.4.1 A typology of roots } & 227\end{array}$

5.4.2 A theory of possible verb classes 231

5.5 Concluding remarks and outlook 233

$\begin{array}{ll}\text { References } & 235\end{array}$

$\begin{array}{ll}\text { Index } & 251\end{array}$ 



\section{General preface}

The theoretical focus of this series is on the interfaces between subcomponents of the human grammatical system and the closely related area of the interfaces between the different subdisciplines of linguistics. The notion of 'interface' has become central in grammatical theory (for instance, in Chomsky's Minimalist Program) and in linguistic practice: work on the interfaces between syntax and semantics, syntax and morphology, phonology and phonetics, etc. has led to a deeper understanding of particular linguistic phenomena and of the architecture of the linguistic component of the mind/brain.

The series covers interfaces between core components of grammar, including syntax/morphology, syntax/semantics, syntax/phonology, syntax/pragmatics, morphology/phonology, phonology/phonetics, phonetics/speech processing, semantics/pragmatics, and intonation/discourse structure, as well as issues in the way that the systems of grammar involving these interface areas are acquired and deployed in use (including language acquisition, language dysfunction, and language processing). It demonstrates, we hope, that proper understandings of particular linguistic phenomena, languages, language groups, or inter-language variations all require reference to interfaces.

The series is open to work by linguists of all theoretical persuasions and schools of thought. A main requirement is that authors should write so as to be understood by colleagues in related subfields of linguistics and by scholars in cognate disciplines.

What exactly roots are has been the subject of intense investigation in generative linguistics for a number of years. In the current volume, Beavers and KoontzGarboden develop a new approach to the meaning of verbal roots. They focus on the interplay between general event structure (which they term templatic meaning), and the idiosyncratic meanings attributed to roots themselves. They argue against theories which take idiosyncratic meaning to be divorced from templatic meaning, arguing, on the basis of a detailed investigation of verb meanings, for a more nuanced relationship between the two and for a richer understanding of the lexical meaning of verbal roots. Their exploration leads them to a new predictive theory of possible verb classes. 



\section{Acknowledgments}

This book is about word meanings, especially the meanings of verbs. It is widely acknowledged that verb meanings have underlying structure, usually consisting

of some fairly general, regular meaning components like cause and change that occur across lots of verbs-the verb's event template-plus some verb-specific idiosyncrasy-the verb's idiosyncratic root. While a considerable amount of work has been devoted to fleshing out the meanings of event templates, less work has focused on the meanings of the idiosyncratic roots that make every verb different, and what kinds of patterns and regularities may be found there. Our goal is to address this question head on.

Although the results here are fairly new, they are rooted in several strands of research which date back nearly two decades. The semantics and morphology of change-of-state verbs and stative forms has been a long-term focus of Andrew's, while the semantics and argument structure of change-of-state verbs and ditransitives has been a long-term focus of John's. These interests were first merged in our initial work on Manner/Result Complementary, i.e. the question of whether a root ever describes both a result and the manner by which the result comes about at the same time. During that collaboration we realized we had each also been independently working within our own case studies on the question of whether roots ever give rise to templatic meanings, i.e. the question of whether root and template meanings are bifurcated. This discovery lead us to ultimately bring all of these strands of work together into the project that gave rise to this book.

Given how long these projects have developed, in both cases going back to our graduate school days, it is almost impossible to ensure that we acknowledge everyone who has given us feedback along the way. We can say without a doubt that we owe an especial debt of gratitude to Beth Levin. The origins of Chapter 2 ultimately lie in a research assistantship Andrew held in his formative graduate school years under Beth, funded by a National Science Foundation grant (BCS0004437) awarded to her, which led to Koontz-Garboden and Levin (2005) and ultimately to work in that chapter and related work. More broadly, Beth has offered extensive feedback and guidance over the years on the various projects tied together in this book (and others), and has more generally served as an inspiration through her own groundbreaking research program, much of it in conjunction with Malka Rappaport Hovav. Their work has helped define the parameters of the field of lexical semantics that we have been working within for most of our careers and has opened up some of the specific questions we address here. We are also enormously grateful to Malka as well for her support and extensive commentary 
and back and forth on the work presented here and related projects over the years, which has been inspiring and helped us sharpen our ideas.

We are also of course grateful to the many people who have given us feedback on this manuscript during its development. This includes the detailed and enormously helpful feedback of three anonymous reviewers of parts of this manuscript, plus the various reviewers of related papers this book draws from and builds on. This material is based upon work supported by the National Science Foundation under grant no. BCS-1451765. We have benefited greatly from extensive discussion with our various project collaborators, including in particular Michael Everdell, Kyle Jerro, Henri Kauhanen, Elise LeBovidge, Stephen Nichols, and Daniel Valle. Special thanks are also due to Ashwini Deo and Itamar Francez for their long term feedback on a variety of issues related to the work here.

We would also like to offer our thanks to various others we've been fortunate to discuss these ideas with, including Luke Adamson, Artemis Alexiadou, Daniel Altshuler, Elena Anagnostopoulou, Nick Asher, Josep Ausensi, Asaf Bachrach, Larry Barsalou, David Basillico, David Beaver, Delia Bentley, Ricardo BermúdezOtero, Nora Boneh, Jonathan Bobaljik, Jurgen Bohnemeyer, Olly Bond, Hagit Borer, Alex Brown, Benjamin Bruening, Ray Buchanan, Lucas Champollion, Beaudry Chase, Liz Coppock, Sergio De Moura Menuzzi, David Embick, Hana Filip, Jens Fleischhauer, Bob Frank, Josep Fontana, Thomas Gamerschlag, Nikki Grant, Heidi Harley, Elena Herberger, Matthew E. Husband, Larry Horn, Ray Jackendoff, E Jamieson, Hans Kamp, Itamar Kastner, Éva Kardos, Graham Katz, Justin Kelly, Chris Kennedy, Paul Kiparsky, Hans van de Koot, Lissa Krawczyk, Jean-Pierre Koenig, Paul Kroeger, Jacques Lafleur, Lisa Levinson, Alec Marantz, Fabienne Martin, Jaume Mateu, Louise McNally, Richard Meier, Victor Mena, Laura Michalis, Ad Neeleman, Andrew Nevins, Johanna Nichols, Tatiana Nikitina, Vitor Nóbrega, Rick Nouwen, Rainer Osswald, Peter Petré, Colin Phillips, Paul Portner, Tillmann Pross, Geoff Pullum, James Pustejovsky, György Rákosi, Gillian Ramchand, Kyle Rawlins, Hannah Rohde, Ivan Sag, Helen Salgi, Florian Schaefer, Jesse Snedecker, Giorgos Spathas, Scott Spicer, Eduardo Soares, Mark Steedman, Peter Svenonius, Alex Teodorescu, Shiao Wei Tham, Lindia Tjuatja, Judith Tonhauser, Christina Tortora, Elly van Gelderen, Robert Van Valin, Sarah Van Wagenen, Klaus von Heusinger, Bonnie Webber, Steve Wechsler, Annie Zaenen, Julia Zinova, Cala Zubair, and Joost Zwarts, among many others.

We are also grateful for the audiences at the 2004, 2006, and 2017 Linguistic Society of America Annual Meetings, the 2006 meeting of the Berkeley Linguistic Society, SemFest 2009, the 2009 Stuttgart Roots Workshop, the 2009, 2010, and 2018 meetings of Linguistic Association of Great Britain, the 2010 meeting of the Georgetown Linguistics Society, Verb Meaning, Event Semantics and Argument Structure 2010, Structuring the Argument 2011, The 5th International Conference on the Linguistics of Contemporary English, Concept Types and Frame 2012, 
the Roots IV Workshop held at New York University in 2015, The University of Texas at Arlington Student Conference in Linguistics and TESOL 2015, the 2016 West Coast Conference on Formal Linguistics, The Society for the Study of the Indigenous Languages of the Americas 2017, Beyond Time 2017, Endpoints 2018, Sinn und Bedeutung 2018, and University of Debrecen, University of Düsseldorf, University of California at Berkeley, University of Göttingen, Universitat Pompeu Fabra, University of Newcastle, and The University of Edinburgh. We likewise thank the participants in Andrew's 2007-14 Grammatical semantics classes and his 2015 Linguistic Society of America Summer Institute course on Approaches to Morphosemantics, and John's Roots of Verb Meaning course at the 2014 Workshop on Formal Linguistics X at Universidade Federal do Rio Grande do Sul in Porto Alegre, Brazil, 2015 Lexical Semantics Seminar at The University of Texas at Austin, and 2015 Linguistic Society of America Summer Institute course on Lexical Semantics at University of Chicago, plus the participants in the University of Texas syntax/semantics research group.

We also owe a special thanks to Julia Steer for her help in making this book a reality, as well as David Adger and Hagit Borer, and also Vicki Sunter for helpful feedback on the manuscript. Thanks also to Michael Yoshitaka Erlewine for some crucial help with $\mathrm{HT}_{\mathrm{E} X}$.

Chapter 4 is a revised and expanded version of Beavers and Koontz-Garboden (2012), originally published in Linguistic Inquiry. We are grateful to The University of Texas Graduate School for support on John's initial work on ditransitives (Beavers 2011a) that later fed this project, The University of Texas College of Liberal Arts for support during the preparation of the initial draft of the full manuscript, and the University of Manchester School of Arts, Languages, and Cultures, for funding several crucial work meet ups. Open access publication of the book has been funded by contributions from National Science Foundation Grant BCS-1451765, the School of Arts, Languages and Cultures at the University of Manchester, the Department of Linguistics and English Language at the University of Manchester, and the University of Manchester Libraries Open Access Fund.

Special thanks are due to Dylan Bumford, Paul DeMello, Kaan Erdener, Shalini Gupta, Damien Harris, Joe McElrath, Chris Micklethwait, Mindy Nobles, Nick Rundquist, Danny Tobey, Judith Tobey, Ted Tran, Juan Valdez, and various others for their judgments and (sometimes lively) discussion of various aspects of the data here and in related projects. Special thanks are also due to Ernest Skočdopole in particular for help with zombies, to Nikki Norman Briscoe, Jennifer Davis, and Jason Cammack for the memory jogs, to everyone at The Railway, Dunham Massey Brewing Co, and Cherrywood Coffeehouse, and to that random guy in Stuttgart who took us to all those dance clubs in the middle of the night back in 2009. 
Finally, our greatest debt of gratitude is of course to Janice, Morrissey, Ezra, Mindy, Melissa, Heath, Blaine, and Lola ${ }^{\dagger}$ for all of their dedication and support, and especially for their tolerance for multiple, extended international work trips, frequent lapses in attention while we were attending to writing, and the occasional noisy late night work sessions that we hope didn't keep everyone up too much. We could not have completed this project without them. 


\section{List of abbreviations}

$\begin{array}{ll}\text { A } & \text { Argument } \\ \text { Adj } & \text { Adjective } \\ \text { AdjP } & \text { Adjective Phrase } \\ \text { Asp } & \text { Aspect } \\ \text { AspP } & \text { Aspect Phrase } \\ \text { COS } & \text { Change of State } \\ \text { DP } & \text { Determiner phrase } \\ \text { FPR } & \text { Figure/Path Relation } \\ i t r & \text { Intransitive } \\ \text { LF } & \text { Logical Form } \\ \text { P } & \text { Preposition } \\ \text { PC } & \text { Property Concept } \\ \text { PF } & \text { Phonetic Form } \\ \text { PP } & \text { Prepositional Phrase } \\ \text { T } & \text { Tense } \\ \text { TP } & \text { Tense Phrase } \\ t r & \text { Transitive } \\ v & \text { Light Verb } \\ v \mathrm{P} & \text { Light Verb Phrase } \\ \text { V } & \text { Verb } \\ \text { VP } & \text { Verb Phrase } \\ \text { XP } & \text { X Phrase } \\ & \\ \end{array}$





\section{Glossing conventions}

$\begin{array}{ll}3 & =3 \text { rd person } \\ \text { ACC } & =\text { accusative } \\ \text { COMP } & =\text { complementizer } \\ \text { DECL } & =\text { declarative } \\ \text { NEG } & =\text { negative } \\ \text { NFUT } & =\text { non-future tense } \\ \text { NOM } & =\text { nominative } \\ \text { OBL } & =\text { oblique } \\ \text { PASS } & =\text { passive } \\ \text { PST } & =\text { past } \\ \text { PFV } & =\text { perfective } \\ \text { REDUPL } & =\text { reduplication } \\ \text { SG } & =\text { singular }\end{array}$





\section{1 \\ Introduction}

\subsection{Lexical semantics and theories of verb meaning}

Lexical semantics is, in a nutshell, the study of the meaning of words (and morphemes), especially in terms of how they relate to other components of language, such as the syntax or morphology. Perhaps the most central theoretical questions in lexical semantics have to do with what constitutes a word's meaning. Is it something wholly atomic, or is a word's meaning decomposed into more basic pieces? Assuming that word meanings are decomposable, what are those components of meaning, and how do they compose together to form larger meanings? Are some of these components universal, and if so, which? For any given theory of word meaning, what kinds of predictions does that theory make about how a given word fits into the grammar of a language, what kinds of meanings words can have, and whether there is any relevant notion of possible and impossible words?

One of the domains where work on these questions has been most richly developed is in the study of verb meaning, a fitting case study since verbs prototypically define the core semantic nucleus of a given clause, whereas other major constituents in the clause, especially those serving core grammatical functions like subject and object, are usually seen as selected dependents of the verb. Perhaps the simplest theory of a verb's meaning is that it describes events and takes arguments naming participants in that kind of event. The combination of that verb with its arguments produces a proposition that is true if an event of that kind with those participants occurred (at the relevant reference time). Thus a verb like kill, to take the famous example of McCawley (1968), describes a killing event and takes first an object that names the entity that dies and then a subject that names the entity that causes the dying, and the combination of those three constituents is true if such an event occurred. To make the nature of the compositional properties more explicit one might furthermore formalize this intuition by defining the meaning of kill in terms of some logical function-defining representation such as in (1) that takes two arguments $x$ and $y$-in that order-and relates them by a relation kill' that is true iff $y$ kills $x$ :

(1) $\llbracket k i l l \rrbracket=\lambda x \lambda y\left[k i l l^{\prime}(y, x)\right]$

(where $\operatorname{kill}^{\prime}(y, x)$ is true iff $y$ kills $x$ ) "The denotation of kill is a function taking individual $x$ and outputting a function taking individual $y$ and outputting True iff $y$ killed $x$." 
Such a theory has predictive value. First, assuming binary functional application the ordering of arguments in (1) correctly ensures that the linguistic expression whose meaning saturates $x$ forms a constituent with the verb first, followed by the linguistic expression whose meaning saturates $y$, e.g. basically the former is the verb's direct object and the latter its subject:

\section{(2) [ Dexter [ killed Brian ] ].}

The meaning in (1) also predicts that (2) will never mean "Brian killed Dexter" since the thing killed must combine with the verb before the thing doing the killing. Finally, this analysis can define a limited theory of arity, where verbs fall into semantically motivated classes in terms of how many arguments they take, e.g. intransitive verbs take one argument, transitive verbs take two, and ditransitive verbs take three.

However, facts such as these are not exhaustive of the linguistically significant aspects of verb meaning. There are many additional facts which require a deeper explanation. First, overwhelmingly across languages, with verbs meaning kill the subject is the killer and the object the entity killed. It is rare that the object is the killer and the subject is the entity killed (Dixon 1994: 8, 111-27) (a type of generalization that emerges from principles such as the Universal Alignment Hypothesis of Perlmutter and Postal 1984: 97 or the Uniform Theta-Role Assignment Hypothesis of Baker 1988: 46). To put it another way, a denotation like (3)which is identical to (1) except that the two arguments are taken in the opposite order-is rarely if ever attested, at least as a basic verb meaning (i.e. modulo passivization or other argument restructuring operations that operate on more basic lexical entries):

(3) $\llbracket k i l l \rrbracket=\lambda y \lambda x\left[k_{i l l}(y, x)\right]$

(where $\operatorname{kill}^{\prime}(y, x)$ is true iff $y$ kills $x$ )

"The denotation of kill is a function taking individual $y$ and outputting a function taking individual $x$ and outputting True iff $y$ killed $x$."

Exceptions to this might include syntactically ergative languages where objects of transitives display syntactic properties of subjects of intransitives (Dixon 1994: 12-7) and Western Austronesian languages with an agent voice vs. object voice contrast (e.g. see Arka 2003: 4-30, 148-62 on Balinese). Otherwise, the main variation seen in such verbs across languages is instead in how and if notions like subject and object are defined and distinguished from one another (e.g. in terms of the properties identified in Keenan 1976). For example, in nominative/accusative languages single argument agreement with transitive verbs is typically triggered by the nominative subject while in ergative/absolutive languages it is typically triggered by the absolutive object, though as per Bobaljik (2008: 302-7) it may be that the determinant of agreement is unmarked surface case rather than some deeper notion of grammatical function. But there are rarely differences in which 
semantic participants for a verb with a meaning like kill bear which grammatical functions, however defined and identified in the language (see Dixon 1994 for a broader discussion of kinds of triggers for different apparent indicators of grammatical function, and especially pp. 131-41, 152-80 on distinguishing a universal notion of subject from a language-particular notion of pivots such as intransitive subject/transitive object pivots).

In other cases there is variation in how arguments are realized, but usually with consistency, as noted in the foundational study of Fillmore (1970). For example, certain arguments have "dibs" for certain grammatical functions when they cooccur with certain other arguments. With break a DP representing the individual causing the breaking can be the subject, but in the absence of such a $\mathrm{DP}$ an instrument with which the breaking is done can be the subject, and absent such a DP the thing broken can be the subject:

(4) a. Mary broke the window (with the hammer).

b. The hammer broke the window.

c. The window broke.

However, not every verb with the same arity is uniform in this behavior. Hit has a meaning similar to break-physical impingement of some entity by some other entity-but disallows uses where the thing hit is the subject:

(5) a. Mary hit the window (with the hammer).

b. The hammer hit the window.

c. ${ }^{*}$ The window hit (with the hammer).

If these were isolated facts about just these two words it might be uninteresting. But most significantly, as Fillmore also noted, for every two verbs that pattern this way there are many more that do the same, i.e. verbs fall into grammatically significant classes. A key difference between break and hit is that the former describes a change of state and the latter some type of surface contact. This is evidenced by the fact that it is a non-cancelable inference of break that something has changed about its object but not of hit, but for hit something must have come into contact with its object but not for break (utilizing a test of contradiction as per Zwicky and Sadock 1975: 7-8): ${ }^{1}$

(6) a. John broke the vase, by singing in a shrill voice/\#but it is undamaged.

b. John hit the vase, but it is undamaged/\#by singing in a shrill voice.

\footnotetext{
${ }^{1}$ Since we are interested in the semantic contribution of the verb to the clause, when testing for the semantics of the verb-i.e. its lexical entailments in the sense of Dowty (1989: 75) - here and throughout we avoid negation and modal operators that would obscure the relevant meaning, and also favor definite, specific DP arguments referring to individuals to avoid mitigating effects of quantification, unless there is a specific reason to introduce one of these complicating factors.
} 
Other verbs describing a change-of-state pattern like break while other surface contact verbs pattern like hit:

(7) a. Kim bent/shattered/cracked the laptop (with the hammer).

b. The hammer bent/shattered/cracked the laptop.

c. The laptop bent/shattered/cracked.

(8) a. Kim slapped/struck/bumped the table (with the hammer).

b. The hammer slapped/struck/bumped the table.

c. *The table slapped/struck/bumped.

This suggests that grammatically significant word classes can be defined by shared semantic properties.

Yet within each broad class of verbs, each verb's meaning differs from all others in the class even if its grammatical behavior in terms of argument realization (or other grammatical facts) does not. For example, as Fillmore noted, different change-of-state verbs describe different specific outcomes for their verbs, giving rise to an interesting effect with nonce objects such as the twarge: different choices of the verb create different impressions about what properties a twarge must have, as in (9):

(9) a. Kim cracked the twarge.

(a twarge has some material integrity)

b. Kim shattered the twarge.

(a twarge is decomposable into bits)

c. Kim bent the twarge.

(a twarge is rigid with some malleability)

Similarly, among change-of-state verbs there is variation in how loose individual verbs are in what can be the causer subject. Break allows humans, instruments, and natural forces, while clear allows only humans and natural forces, and murder only humans: ${ }^{2}$

(10) a. Leah/The earthquake/The hammer broke the lamp.

b. Leah/The heavy winds/\#The broom cleared the patio of leaves.

c. Leah/\#The earthquake/\#The hammer murdered the victim.

Thus there are semantic generalizations in how arguments are mapped to syntax, awash in a sea of individual idiosyncrasy. An adequate theory of verb meaning

${ }^{2}$ The broom cleared the patio of leaves is possible for some speakers if the broom is interpreted as a true instrument, i.e. if there is an implicit agent operating it. In this case it is akin to a middle construction (e.g. These cars sell easily), in which the verb's default causer/agent argument is still entailed to exist but is left unexpressed and is crucially no longer associated with the subject grammatical function. Thus there are no relevant selectional restrictions on the instrument, which is instead realized as subject (see also so-called adjunct middles in Dutch Ackema and Schoorlemmer 1994: 82-8, 2004: 146-7). 
(and word meaning in general) must therefore be able to identify (a) the broader aspects of verb meaning that grammar is sensitive to and (b) the principles that relate them to the grammar while (c) admitting some degree of individual semantic variation on a verb-by-verb basis. Key here is that the actual truth conditional content and aspects of the meaning beyond just the arity and order of arguments are relevant. In other words, it is not sufficient to define the semantic relation of $x$ and $y$ in (1) as some function that returns true iff $y$ kills $x$, leaving the notion of "kill" undecomposed. A better theory of word meaning must consider the more basic components that make up situations of killing so as to explain such data.

Much theorizing in lexical semantics over the last several decades has thus sought to elucidate the subcomponents of verbal meaning relevant for defining a verb's grammatical behavior and inference patterns in predictive ways. The central focus of the vast majority of this work has been on the broader, more regular aspects of verbal meaning that define verb classes. Somewhat less attention has been paid to the more idiosyncratic aspect of verb meaning, the ones that distinguish verbs within a class and derive inferences such as those in (9) and acceptability patterns such as those in (10). Of course, it is understandable that the field has developed in this way: while the idiosyncratic semantics that derive facts such as those in (9) and (10) are not without lexicographic interest, much that we could learn about such meanings will definitionally not generalize much. If the bulk of the verb's grammatical properties are rooted in the verb class-level semantics then this is the area that presumably has more to say about how lexicons and thus grammars of languages are organized more generally.

The central goal of this book is to look more closely at the supposed lexical idiosyncrasy within verb classes - the unique aspect of meaning that distinguishes verbs within a class-and to ascertain whether there are broad, significant generalizations to be found within these verb-specific components that have semantic and grammatical consequences. Our central thesis is that the verb-specific portions of a verb's meaning themselves also fall into systematic and regular classes and that, crucially, these classes are defined in terms of the same sorts of semantic properties that define broader verb classes, e.g. classes that center around such notions as whether the idiosyncratic, verb-specific meanings involve a change of state or surface contact, albeit defined entirely independent of and thus technically orthogonal to the verb class. We will furthermore suggest that these verb-specific aspects of a verb's meaning are grammatically relevant in ways similar to the verb's broader semantic class. Thus our goal is to demonstrate that there are essentially two well-springs of regularity within the lexicon, one arising from broad semantic classes of verbs and one arising from the verb-specific aspects of verbal meaning, a crisscrossing of which provides a more nuanced theory of what a verb can mean than has been apparent on prior approaches.

However, in order to outline our major research questions, it will help to first outline a framework of analysis that separates the more regular verbal meanings 
from the more verb-specific ones. The approach we adopt here is the so-called "event structural" approach to verb meaning that takes verb meanings to be defined in terms of structured classifications of the events they describe. This approach has a long and fruitful history in theories of lexical semantics, and is perhaps the dominant approach to the study of verbal meaning today (see e.g. Lakoff 1965; McCawley 1968, 1971; Ross 1972; Dowty 1979; Pinker 1989; Jackendoff 1990b; Hale and Keyser 1993, 1997, 2002; Goldberg 1995; Levin and Rappaport Hovav 1995; Pesetsky 1995; Baker 1997; Marantz 1997; Wunderlich 1997; Van Valin and LaPolla 1997; Rappaport Hovav and Levin 1998; Davis and Koenig 2000; Davis 2001; Folli and Ramchand 2002; Harley 2003; Folli and Harley 2004; Borer 2005, 2013; Grimshaw 2005; Ramchand 2008; Rappaport Hovav and Levin 2010; Mateu and Acedo-Matellán 2012; Alexiadou et al. 2015; inter alia, much of this work also building on work that has proposed classifications of events and/or verbs based on types of events described independent of particular decompositional analyses as per Vendler 1957; Verkuyl 1972; Bach 1986; inter alia). The bulk of this chapter is thus devoted to outlining and justifying such a theory of verb meaning and how it captures the kinds of facts discussed above, plus more. First, though, we briefly discuss an alternative to event structural approaches that takes classifications of event participants rather than events to be the key feature of classifying verb meaning, since insights from this approach have directly fed work in event structural approaches.

\subsection{Thematic roles as a theory of verb meaning}

One early proposal for a principled theory of verb meaning (Gruber 1965; Fillmore 1968, 1970; Jackendoff 1972) is that the grammatically significant meaning of a verb consists of a list of "thematic roles" that define what a given participant in the event described by the verb is doing. Such roles are furthermore drawn from a crosslinguistically universal set that cuts across verbs, such as agent (the instigator of an action), patient/theme (an entity that changes, moves, or comes into/goes out of existence; we use "patient" and "theme" interchangeably), location (an entity at which something is located), and instrument (an intermediate entity an agent uses to affect a patient). Verbs in the same semantically-defined grammatical classes assign the same thematic roles to their arguments, which in turn serve as the basis for supposedly universal "linking rules" that constrain the possible grammatical configurations a verb can occur in. For example, if kill takes an agent and patient then if (11) define universal principles relating these thematic roles to grammatical functions, it determines that (1) is a possible denotation for kill but (3) is not:

(11) a. If the verb has an agent argument, it is the subject.

b. If the verb has a patient argument, it is the object. 
Such rules can be viewed as online rules of linking meanings to the syntax (e.g. as per Fillmore 1968: 34-78), or as principles guiding how people lexicalize meanings into verbs (e.g. as per Dowty 1991: 576). Either way, the facts about possible change-of-state verbs in terms of how they takes their arguments will follow. However, rules such as (11) are perhaps too rigid since, as we saw in (4), agents are not the only thematic roles that can be assigned to the subject of a sentence. Instruments and patients can also be subjects, albeit only in certain contexts (Fillmore 1970: 122-5). These rules do not really take those additional possibilities directly into account.

A key further insight of thematic role approaches (dating to at least Fillmore 1968: 33) is that thematic roles themselves may be related to one another hierarchically as part of a universal theory of word meaning, and linking rules are sensitive to relationships between thematic roles. Thus the data in (4) can be explained by assuming a universal ranking of thematic roles that puts agents first, instruments next, and patients last, with a more general linking rule than the one in (11a) that assigns the bearer of the most prominent thematic role in a verb's meaning the subject grammatical function:

a. agent $>$ instrument $>$ patient

b. The argument with the highest thematic role is the subject.

On such an analysis hit and other surface contact verbs would have a different set of thematic roles, in particular agent and location, and if locations are not part of the hierarchy for subject selectional preferences then a location-subject version of the verb should be ruled out. The key emergent observation is that grammatically relevant lexical meaning is not just universal but structured-there are linguistically significant relationships between lexical semantic primitives that correspondences relating lexical semantics to syntax are sensitive to. In the case of thematic role theories the key universal components are participant types, which can be used to define equivalence classes of verbs, and the key lexical semantic structures are thematic role hierarchies.

Subsequent work in thematic roles has led to richer theories rooted in these basic primitives. For example, it is sometimes assumed that in addition to thematic roles and rules linking them to syntax there is a need for some intermediate level of grammatical information sometimes called a "predicate argument structure" that defines phrase structure-independent aspects of a verb's argument realization properties (see e.g. Rappaport and Levin 1988). Similarly, a simple theory that defines thematic roles as atomic labels shared across verbs does not necessarily explain how they relate to inferences such as those in (6), nor does it explain the idiosyncratic semantic properties of individual verbs such as those in (9). However, work in the tradition of Dowty $(1989,1991)$ (see e.g. Primus 1999; Ackerman and Moore 1999a,b, 2001; Beavers 2006, 2010) has resolved this 
problem by defining thematic roles not as atomic labels but as sets of lexical entailments (Dowty 1989: 75). The relevant entailments may be quite verb specific (Dowty's 1989: 76 “individual thematic roles”). Linking principles can be defined on the intersections of idiosyncratic thematic roles (Dowty's 1989: 77 "thematic role types"), thus applying to verbs sharing semantic components in common, recreating the types of general thematic role labels discussed above.

Nonetheless, despite many of these innovations thematic role approaches to word meaning are inherently limited (see Rappaport and Levin 1988; Dowty 1989; Wechsler 1995: 7-15; Levin and Rappaport Hovav 2005: 38-49, 154-85, Beavers 2006: 238-242). First, it has been difficult to determine the right number and "grain size" of thematic roles, so that identifying a universal, linguistically significant set of them has proven elusive. Furthermore, on thematic role approaches it is sometimes difficult to derive which combinations of thematic roles define possible verbs and which do not. For example, while verbs taking just an agent and a patient are common, verbs taking (say) just a location and an instrument are not. Partly the problem has to do with the fact that thematic roles are generally defined independently of one another and thus there is in principle no reason why any combination of thematic roles cannot form the basis of a particular verb class. Third, thematic role hierarchies are often independently unmotivated theoretical constructs (though see Givón 1984: 199-201 and Levin and Rappaport Hovav 2005: 174-5), and many hierarchies proposed in the literature are mutually incompatible. For example, in a survey of various proposals for supposedly universal thematic role hierarchies Levin and Rappaport Hovav (2005: 162-3) show that other than a tendency to assume that agent is very highly ranked, there is almost no consensus on how other roles are ranked. For these reasons an alternative view of verb meaning is now more common, rooted not in classifications of the participants of the events the verbs describe but instead in terms of the types of events themselves.

\subsection{Event structures as a theory of verb meaning}

\subsubsection{Roots, templates, and decompositional structure}

An observation dating back to at least Lakoff (1965) is that verbal meanings are often roughly (if not precisely) paraphrasable by analytic constructions that make plain certain basic subcomponents of how the events they describe unfold. For example, the transitive change-of-state verb flatten in (13a) is subject to the near paraphrase in (13b), which highlights the structure of the event it describes as an (action on the part of an) agent that caused a change:

(13) a. Mary flattened the rug.

b. Mary caused the rug to become flat. 
Intransitive flatten in (14a) instead has the near paraphrase (14b), which effectively constitutes a portion of (13b):

(14) a. The rug flattened.

b. The rug became flat.

Other change-of-state verbs are subject to nearly identical paraphrases regarding the unfolding of the event, differing only in what the final state is:

(15) a. Mary dried the rug.

b. Mary caused the rug to become dry.

(16) a. The rug dried.

b. The rug became dry.

Conversely, paraphrases of intransitive jog and run emphasize an action and not a change of state, differing only in which action each verb describes:
a. Mary jogged/ran.
b. Mary did jogging/running actions.

Crucially, the paraphrases do not just highlight the kinds of events each verb describes but decompose them into more basic subevents. These include frequent and recurring event types such as "action," "cause," and "become" that differentiate whole classes of verbs, with more specific notions like "flat," "dry," "jogging," and "running" differentiating verbs within these classes.

Building on such observations, a considerable body of work has proposed that the grammatically significant aspects of a verb's meaning consists of some type of event structure. An event structure is a level of representation associated somehow with a surface verb relative to which generalizations relating a verb to its grammatical properties can be defined. Event structures consist of two basic components. The first is an event template built of some universal set of grammatical primitives defining basic event types such as action, causation, and change, taking arguments filling in standard participants in those events to define the event's broad temporal and causal contours. Crucially, some of these primitives may take event templates as arguments, building larger event templates out of smaller ones. The second component of an event structure is an idiosyncratic, verb-specific root (or constant) that describes real world actions (e.g. jogging) and states (e.g. hungry, dead) that distinguish verbs with the same template from one another either by serving as arguments to eventive primitives or modifying them in some way. Thus a (sense of a) verb is the association of an idiosyncratic surface morphological stem with a denotation (partly) derived from an event template and an idiosyncratic root that comprise a complete event structure. 
For example, using the notation adopted by Rappaport Hovav and Levin (1998: 108) (rooted in Dowty 1979: 71-129), event structures are semantic representations associated with a particular verb built of basic non-logical constant primitives such as ACT, BECOME, and CAUSE defining action, becoming, and causing, respectively. We can define jog and the two uses of flatten as in (18). Here jog defines a type of ACT event qua an action by some entity $x$, where the root specifies that it is a jogging sort of action. Intransitive flatten is a BECOME event qua change of $x$ into a flatness state defined by its root, while the transitive variant is the same event structure embedded as an argument of CAUSE that also takes an additional causer argument $y:^{3}$
a. jog, $\left[y \mathrm{ACT}_{<\text {jogging }>}\right]$
b. flatten $_{\text {itr }},[x$ BECOME $<$ flat $>$ ]
c. flatten $_{t r},[y$ CAUSE $[x$ BECOME $<$ flat $>]]$

The difference between jog and run or the two variants of flatten and the two variants of $d r y$ would have to do solely with the root component of the event structure; the event templates are identical:
a. run, $\left[y \mathrm{ACT}_{<\text {running }>}\right]$
b. $d r y_{i t r},[x$ BECOME $<$ dry $>]$
c. $d r y_{t r},[y$ CAUSE $[x$ BECOME $<$ dry $>$ ] ]

The division between templates and roots captures the distinction between verb classes (defined by templates) and individual verbs within a class (defined by roots) in the same way thematic role types vs. individual thematic roles did for thematic role theories. Once the meanings of the relevant components of the event structure and their means of composition are defined (see below) then the correlation of the verb classes to semantic entailments such as change of state as in (6) follows from the meanings of the event templates, while idiosyncratic verb-specific properties such as those in (9) and (10) follow from the meaning of the root.

Furthermore, a key idea in event structural approaches is that (the structures of) templates are linguistically significant, figuring into the various syntactic, morphological, and modificational behaviors that define verb classes. For example, variable argument realization facts such as those in (4) can be defined in terms of principles of argument realization that reference the relative position of different arguments in the event structure. Roughly, how embedded an argument is in the event structure correlates with how embedded it is in the phrase structure projected by the verb (see e.g. Wunderlich 1997: 43-53 or Van Valin and LaPolla

\footnotetext{
${ }^{3}$ This discussion simplifies the complexity of the causative/inchoative alternation, particularly crosslinguistically. Although this may be the right kind of analysis for English, it seems likely it is not for other languages. See $\$ 2.2 .1$ for discussion and references.
} 
1997: 139-47; see also Levin and Rappaport Hovav 1995: 135-54, who define realization rules relating arguments in specific places in event structures with specific grammatical realizations). If we treat event structures as translations of verbs into some logical representation language within a typed lambda-calculus (see e.g. Dowty 1979: 200-5) we might define linking rules by which the order in which arguments are lambda-abstracted over for any lexeme require the most embedded argument to be the first argument to be saturated and the highest the last:
a. $\llbracket$ flatten $_{t r} \rrbracket=\lambda x \lambda y[y$ CAUSE $[x$ BECOME $<$ flat $>$ ] ]
b. $\llbracket$ flatten $_{\text {itr }} \rrbracket=\lambda x[x$ BECOME $<$ flat $>]$

Given this, that caused change-of-state verbs have agent subjects and patient objects follow from the basic architecture-the agent is less embedded in the event structure than the patient and thus surfaces higher in the syntax, ruling out a version of such a verb like that in (3). Furthermore, the variable argument realization facts in (4) follow from the relationship of (20a) to (20b) (leaving aside instrument subjects): since the former embeds the latter the former will have its $x$ argument lower than its $y$ argument and thus not accessible to being the highest argument syntactically. But absent the causation structure in (20b) the $x$ argument is the highest. ${ }^{4}$ Furthermore, the lack of inchoative hit might follow from its having an event structure different from that of flatten in (20a) that lacks the requisite structure to produce the kind of embedded forms in (20b). This approach can also capture grammatical facts that are more difficult to capture on thematic role approaches. For example, in many languages basic eventive notions such as causation in a verb's meaning give rise to systematic morphological properties of the verb, e.g. causative morphemes in languages that have those. On the event structural approach this correlation could be derived by correspondence rules between the templatic operators within a verb's event structure and its morphological form.

As presented so far an event structure is a level of representation independent of the syntax-for example stored in the lexical entry of the verb-that determines the verb's grammatical properties through linking rules of some type. This is not the only way to conceive of an event structure. For some proponents of this type of theory the correlation of templates to the syntax-especially the correlation of embeddedness of an argument in the event structure to its embeddedness in the syntax-is so tight that an event structure is instead taken to be the syntax

\footnotetext{
4 That said, these results do depend on the various event templatic operators taking their arguments in exactly this order. Nothing in principle rules out CAUSE taking the causer as its second argument and the BECOME event structure as its first, which might change the embedding in ways that would reverse the syntactic argument asymmetries (see Dowty 1979: 95-6, Koenig and Davis 2006, and Beavers 2010: 827-8 for further discussion). However, given the common assumptions about how such templatic operators work these results follow.
} 
(Lakoff 1965; McCawley 1968, 1971; Ross 1972; Hale and Keyser 1993, 1997, 1998, 2002; Pesetsky 1995; von Stechow 1996; Baker 1997; Marantz 1997; Travis 2000; Harley 2003; Folli and Ramchand 2002; Folli and Harley 2004; Alexiadou et al. 2006; Ramchand 2008; Alexiadou et al. 2015; inter alia). ${ }^{5}$ On such approaches, exemplified most prominently in work in Distributed Morphology (DM) (Halle and Marantz 1993), a surface verb is analyzed underlyingly as a phrase structure in which the event template is defined by projections of functional heads such as light verbs (e.g. v), possibly realized overtly as derivational morphemes (e.g. -en/ed for $v_{\text {become }}$, capturing the correspondence between regular morphology and event structures noted above). The event structural root is an idiosyncratic morphological root that modifies or serves as an argument to a $v$, with the root often treated as acategorial (with $v$ providing the category), though nothing we say here hinges on this issue at all:

(21) a. Mary jogged.

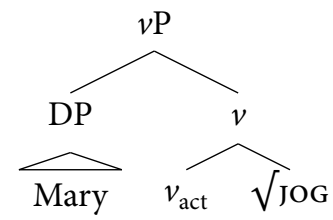

b. The rug flattened.

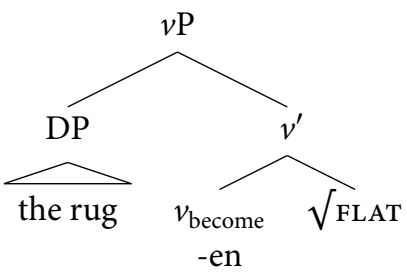

c. Mary flattened the rug.

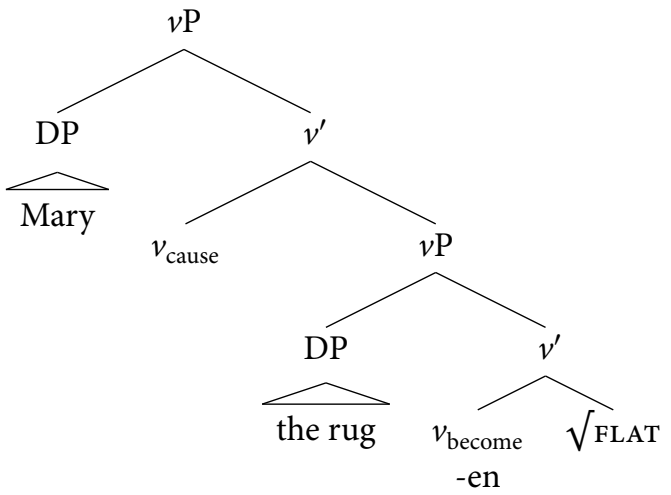

\footnotetext{
${ }^{5}$ A closely related alternative, as seen in some Construction Grammar approaches (e.g. Goldberg 1995 or Iwata 2005a,b, 2008), has a distinction between constructions and lexemes where each contributes event structural information, though the division of labor does not necessarily map onto the root vs. template distinction as it does with the approaches cited above (see fn. 12).
} 
Some processes of head movement ensure that the various morphological pieces are concatenated to form a single surface verb. On this view no linking rules or other rules relating event structures to the grammatical properties of the verb are required. That the agent is the highest argument follows because $v_{\text {cause }}$ and $v_{\text {act }}$ are the light verbs that take agents and since they are always at the top of the event structure the agent will be the highest argument (though similar stipulations about the order of arguments $v_{\text {cause }}$ and $v_{\text {act }}$ take as discussed for CAUSE in fn. 4 are required for this analysis to work). If this is a universal fact about such heads then this can account for the absence of patient-subject/agent-object verbs as per (3). In the absence of $v_{\text {cause }}$ in (30b) the highest argument is the patient, capturing the variable argument realization facts. However, while event structural approaches can capture the same facts as thematic role approaches, it is in their semantics that they provide additional predictions that are more difficult to capture with thematic roles. We turn to this next.

\subsubsection{A semantics for event structures}

A major question that arises for event structures is how exactly the event structure constrains the meaning of the (surface) verb-what are the individual truth conditional contributions of the various component pieces and how are they composed? This is an important theoretical question: without a theory of the meanings of an event structure's component pieces, no predictions are necessarily made about what kinds of meanings verbs can have. If there are no constraints, any event structure could have any meaning. Indeed, nearly all of the above predictions were about the verb's grammatical aspects, and they follow mainly from the structure of the event template-the choice of and hierarchical arrangement of its functional heads-not its meaning. While those facts may correlate with some facts about the verb's meaning, we have not yet derived a theory of what a verb can mean such that any correlations-or much semantics at all-follows from event structures.

There are a number of ways of going about developing a theory of event structural meaning, and it is immaterial what method one chooses since many of the questions about how event structures contribute to meaning can be stated analogously in different implementations. However, for concreteness we present one approach here, rooted in the fully syntactic event structures of (21), and show that it makes additional predictions about verb meaning that suggest the overall correctness of the approach (though see $\$ 1.6$ for some refinements). We represent the meanings of functional heads and roots as translations into a formal logical language that can in turn be interpreted model theoretically (though we do not go through the details of this step). We focus on an analysis of the meanings of causative and inchoative change-of-state verbs that illustrate the advantages of having an explicit semantics and will also set the stage for the analyses to be presented in subsequent chapters. 
We assume a domain of discourse $U$ that consists of two major sorts: the set $U_{I}$ of individuals and the set $U_{V}$ of eventualities. $U_{V}$ is further subdivided into events $U_{E}$ and states $U_{S}$, also two different sorts. For individuals we use variables $x, y, z$, for eventualities (events or states) $e, v, h$, for states proper $s$, for higher order types $P, Q$, and $R$, and mnemonic boldface symbols for logical constants (mainly for translations of definite DPs), all possibly with primes, superscripts, and subscripts. ${ }^{6}$ To derive logical meaning translations for surface verbs we assign the denotations such as the following to the various functional heads and roots, which will sometimes take event arguments and sometimes state arguments. In this way, roots have types, much as in the work of Levinson (2007), Henderson (2019), and Coon (2019), which have consequences for the kinds of grammatical contexts they can appear in. ${ }^{7}$ For the moment we treat the truth conditions for holding a state, change-of-state, and causation fairly informally; we return in more detail to these in $\$ 1.6{ }^{8}$
a. $\llbracket \sqrt{ }$ FLAT $\rrbracket=\lambda x \lambda s\left[\right.$ flat $\left.^{\prime}(x, s)\right]$
"A state of flatness $s$ holds of $y$."
b. $\llbracket v_{\text {become }} \rrbracket=\lambda P \lambda x \lambda e \exists s\left[\right.$ become $\left.^{\prime}(s, e) \wedge P(x, s)\right]$
"Event $e$ gives rise to a state $s$ of type $P$ for individudal $x$."
c. $\llbracket v_{\text {cause }} \rrbracket=\lambda Q \lambda y \lambda v \exists e\left[\right.$ effector $\left.^{\prime}(y, v) \wedge \operatorname{cause}^{\prime}(v, e) \wedge Q(e)\right]$
"Event $v$ with $y$ as its effector causes an event $e$ of type $Q$."

We must also make a few additional assumptions. We assume a transformational syntax that ultimately produces a Logical Form (LF) and a Phonetic Form $(\mathrm{PF})$, and we assume heads and individual denoting DPs that undergo headmovement and A-movement for syntactic reasons respectively reconstruct at LF (or if movement leaves behind copies, they are interpreted at their first Merge position). We largely ignore $\mathrm{A}^{\prime}$-movement save that we assume quantificational DPs undergo Quantifier Raising, leaving behind traces or copies of type $e$ that

${ }^{6}$ As it emerges below we will sometimes make reference to pure states, but rarely to pure events, and thus most event variables will be of the eventuality sort. For this reason we will often use the term "event" loosely to refer to eventualities unless there is reason to be especially clear about the event vs. eventuality distinction.

${ }^{7}$ That said, these types play no role in distinguishing between the classes of root we investigate in the chapters below. All the roots of transitive change-of-state verbs in Chapters 2 and 4, for example, are of the same formal type, as are the roots of ditransitives discussed in Chapters 3 and 4 . So it must be something else that distinguishes between them. That something else amounts to systematic differences in each case in the idiosyncratic truth conditional contribution of the root.

8 We assume that causing eventualities and eventualities of change can be states as well as events as per Beavers and Zubair (2013) in the former case and Gawron (2009) and Koontz-Garboden (2010) in the latter case; see $\$ 2.2 .2$ for more. Following Van Valin and Wilkins (1996) we use the generic term "effector" as the thematic role for causer arguments. 
represent open variables to be bound later by the raised DP, though we generally only use non-quantificational DPs. We assume that any unsaturated event variables are existentially quantified over at TP (Higginbotham 1985: 560-1), though we generally ignore functional projections above $v \mathrm{P}$ as they are not of interest here, and mostly present logical representations of sentences that lack tense information, grammatical aspectual information, or discourse-related information.

The inchoative (21b) thus has a compositional meaning as follows:
a. $\llbracket\left[-e n_{v_{\text {become }}} \sqrt{\text { FLAT }}\right] \rrbracket$
$=\llbracket-e n_{v_{\text {become }}} \rrbracket(\llbracket \sqrt{\text { FLAT }} \rrbracket)$
$=\lambda x \lambda e \exists s\left[\operatorname{become}^{\prime}(s, e) \wedge \operatorname{flat}^{\prime}(x, s)\right]$
b. $\mathbb{[}[$ the rug $]\left[-e n_{v_{\text {become }}} \sqrt{\text { FLAT }]}\right] \rrbracket$
$=\llbracket\left[-e n_{v_{\text {become }}} \sqrt{\text { FLAT }] \rrbracket(\llbracket[\text { the rug }] \rrbracket)}\right.$
$=\lambda e \exists s\left[\operatorname{become}^{\prime}(s, e) \wedge\right.$ flat $\left.^{\prime}(\mathbf{r}, s)\right]$
(functional application)
c. $\exists e \exists s\left[\operatorname{become}^{\prime}(s, e) \wedge\right.$ flat $\left.^{\prime}(\mathbf{r}, s)\right]$
(existential closure)
"There is an event $e$ of change giving rise to a state $s$ of flatness holding of the rug."

Here $v_{\text {become }}$ introduces entailments of change as in (6), while the root $\sqrt{\text { FLAT }}$ derives individual inferences and constraints as in (9) and (10). Causative (21c) embeds (23b) and adds a causing event and effector:
a. $\llbracket\left[v_{\text {cause }}\left[[\right.\right.$ the rug $\left.\left.]\left[-e n_{v_{\text {become }}} \sqrt{\text { FLAT }}\right]\right]\right] \rrbracket$
$=\llbracket v_{\text {cause }} \rrbracket\left(\llbracket\left[[\right.\right.$ the rug $\left.\left.]\left[-e n_{v_{\text {become }}} \sqrt{\text { FLAT }}\right]\right] \rrbracket\right)$
(functional application)
$=\lambda y \lambda v \exists e\left[\operatorname{effector}^{\prime}(y, v) \wedge \operatorname{cause}^{\prime}(v, e) \wedge \exists s\left[\operatorname{become}^{\prime}(s, e) \wedge\right.\right.$ flat $\left.\left.^{\prime}(\mathbf{r}, s)\right]\right]$
b. $\mathbb{[}[$ Mary $]\left[v_{\text {cause }}\left[[\right.\right.$ the rug $\left.\left.\left.]\left[-e n_{v_{\text {become }}} \sqrt{\text { FLAT }}\right]\right]\right]\right] \rrbracket$
(functional application)
$=\llbracket\left[v_{\text {cause }}\left[[\right.\right.$ the rug $\left.\left.]\left[-e n_{v_{\text {become }}} \sqrt{\text { FLAT }}\right]\right]\right] \rrbracket(\llbracket[$ Mary $] \rrbracket)$
$=\lambda v \exists e\left[\operatorname{effector}^{\prime}(\mathbf{m}, v) \wedge \operatorname{cause}^{\prime}(v, e) \wedge \exists s\left[\operatorname{become}^{\prime}(s, e) \wedge\right.\right.$ flat $\left.\left.^{\prime}(\mathbf{r}, s)\right]\right]$
c. $\exists v \exists e\left[\operatorname{effector}^{\prime}(\mathbf{m}, v) \wedge \operatorname{cause}^{\prime}(v, e) \wedge \exists s\left[\operatorname{become}^{\prime}(s, e) \wedge\right.\right.$ flat $\left.\left.^{\prime}(\mathbf{r}, s)\right]\right]$
(existential closure)
"There is an event $v$ of which Mary is the effector and $v$ causes an event $e$ which is an event of change that gives rise to a state $s$ of flatness that holds of the rug."

This analysis makes two key predictions. First, the decompositional analysis predicts that the causative variant should entail the corresponding inchoative variant (barring any quantification or modality that we ignore here; see \$1.6.2.1). 
In particular, the final meaning in (24c) entails the final meaning in (23c) by simple virtue of the fact that the head that projects the phrase corresponding to the latter takes the phrase defining the former as an argument and conjoins in the extra information that there is a cause for the event.

Second, this analysis also provides a way of capturing the phenomenon of sublexical modification, i.e. the fact that certain classes of modifiers can target subparts of the event structure. For example, again, when applied to verbs with complex event structures, allows multiple interpretations. Thus Mary flattened the rug again has at least three readings, namely the restitutive reading that Mary is restoring the rug to a state of flatness that it had before and at least two repetitive readings that Mary is causing the rug to undergo a flattening that it had undergone before (that may have had no cause or may have been caused by something or someone other than Mary) or that Mary is repeating her own previous flattening of this rug:

(25) Mary flattened the rug again
a. .... and it had been flat before.
b. ... and it had flattened before.
c. ... and Mary had flattened it before.

(restitutive)

(repetitive)

(repetitive)

There is a hierarchical relationship between these readings: the reading in (25c) entails that in (25b), which entails that in (25a), i.e. for Mary to have flattened the rug before entails it had flattened before, which entails that it was flat before. The ambiguity and the entailment relationship between the readings is neatly predicted by (24) if (25) reflects an attachment ambiguity over how much of the event structure again takes scope over: the root, a projection of $v_{\text {become }}$ (which contains the root), or a projection of $v_{\text {cause }}$ (which contains $v_{\text {become }} \mathrm{P}$ ). Since this modifier will be of particular interest in this study we work through the details of the analysis. If again is defined as in (26) (adopting a simplified version of von Stechow's 1996: 95 analysis; see also Beck and Johnson 2004: 107, (21); von Stechow 1995, 2003; Beck 2006; Marantz 2007, 2009) then the three readings follow from attachment at the three levels in (27) (here we omit the compositional details): ${ }^{9}$

$$
\llbracket \text { again } \rrbracket=\lambda P \lambda z \lambda e^{\prime \prime \prime}\left[P\left(z, e^{\prime \prime \prime}\right) \wedge \partial \exists e^{\prime \prime}\left[e^{\prime \prime} \ll e^{\prime \prime \prime} \wedge P\left(z, e^{\prime \prime}\right)\right]\right]
$$

${ }^{9}$ For eventualities $e^{\prime \prime \prime}$ and $e^{\prime \prime}, e^{\prime \prime} \ll e^{\prime \prime \prime}$ is true iff $e^{\prime \prime}$ occurred before $e^{\prime \prime \prime}$. We assume with Beck (2006: 278-80) and Deo et al. (2011: 111-2), inter alia, that the content again introduces is presuppositional, marking it with the $\partial$ operator of Beaver (1992: 4-5) that introduces presuppositions, though the details of an analysis of presupposition are not our concern here. 
(27) a.

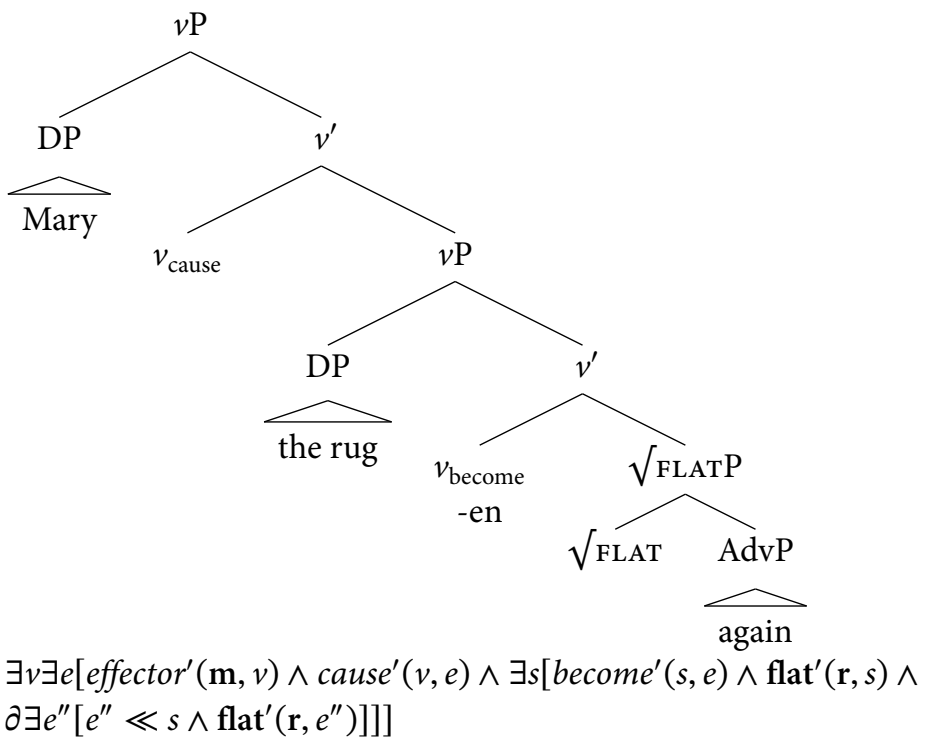

"There is an event $v$ of which Mary is the effector and $v$ causes an event $e$ which is an event of change that gives rise to a state $s$ of flatness that holds of the rug and there is presupposed to be an earlier state $e^{\prime \prime}$ of flatness that held of the rug."

b.

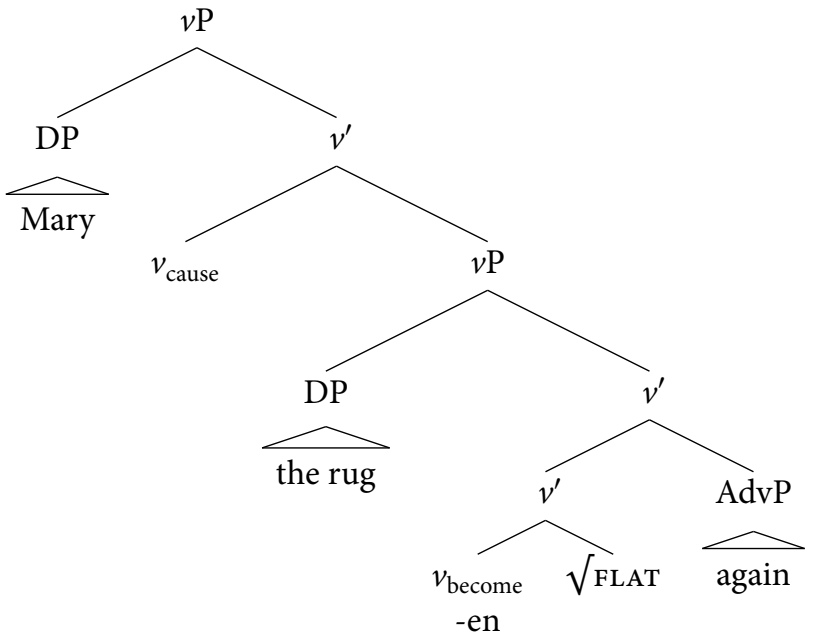

$\exists v \exists e\left[\operatorname{effector}^{\prime}(\mathbf{m}, v) \wedge \operatorname{cause}^{\prime}(v, e) \wedge \exists s\left[\right.\right.$ become $^{\prime}(s, e) \wedge$ flat $\left.^{\prime}(\mathbf{r}, s)\right] \wedge$ $\partial \exists e^{\prime \prime}\left[e^{\prime \prime} \ll e \wedge \exists s\left[\operatorname{become}^{\prime}\left(s, e^{\prime \prime}\right) \wedge\right.\right.$ flat $\left.\left.\left.^{\prime}(\mathbf{r}, s)\right]\right]\right]$

"There is an event $v$ of which Mary is the effector and $v$ causes an event $e$ which is an event of change that gives rise to a state $s$ of flatness that holds of the rug and there is presupposed to be an earlier event $e^{\prime \prime}$ which is an event of change that gave rise to a state $s$ of flatness that held of the rug." 
c.

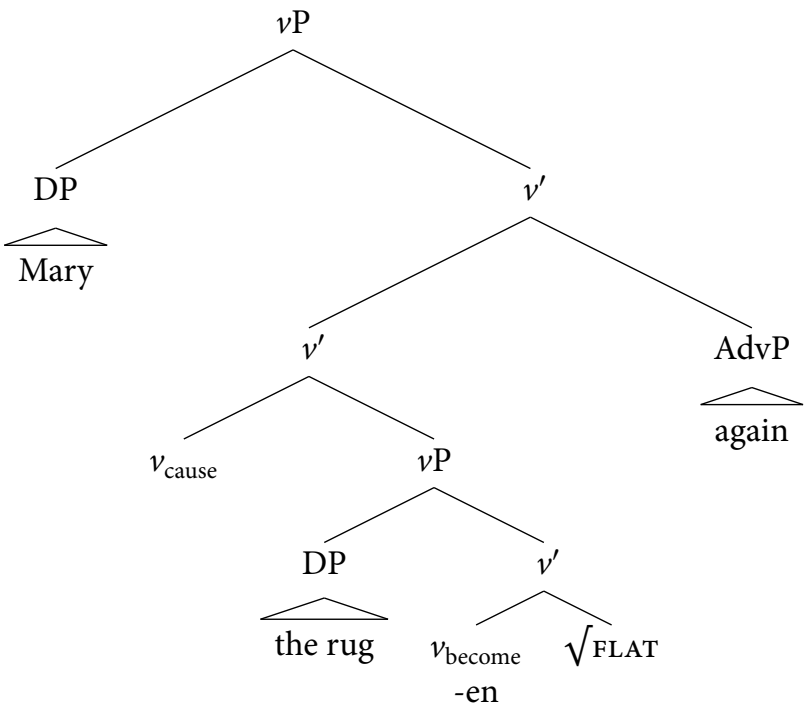

$\exists e^{\prime \prime \prime}\left[\exists e\left[\right.\right.$ effector $^{\prime}\left(\mathbf{m}, e^{\prime \prime \prime}\right) \wedge$ cause $^{\prime}\left(e^{\prime \prime \prime}, e\right) \wedge \exists s\left[\right.$ become $^{\prime}(s, e) \wedge$ flat $\left.\left.^{\prime}(\mathbf{r}, s)\right]\right] \wedge$ $\partial \exists e^{\prime \prime}\left[e^{\prime \prime} \ll e^{\prime \prime \prime} \wedge \exists e\left[\right.\right.$ effector $^{\prime}\left(\mathbf{m}, e^{\prime \prime}\right) \wedge$ cause $^{\prime}\left(e^{\prime \prime}, e\right) \wedge \exists s\left[\right.$ become $^{\prime}(s, e) \wedge$ flat $\left.\left.\left.\left.^{\prime}(\mathbf{r}, s)\right]\right]\right]\right]$

"There is an event $e^{\prime \prime \prime}$ of which Mary is the effector and $e^{\prime \prime \prime}$ causes an event $e$ which is an event of change that gives rise to a state $s$ of flatness that holds of the rug and there is presupposed to be an earlier event $e^{\prime \prime}$ of which Mary is the effector and $e^{\prime \prime}$ caused an event $e$ which is an event of change that gave rise to a state $s$ of flatness that held of the rug."

This theory also predicts that the root at the bottom will define an unanalyzable scopal unit. Thus Mary flattened the rug again on the lowest scope of again means that the rug again meets the entire set of truth conditions that go with flatness (e.g. that all of it be flat, for example). There is no further accessible decomposition of the meaning of the root. We return to this point and its significance again in $\$ 1.4 .3^{10}$

There are other possible sublexical modifiers that show similar effects. For example, for an hour temporal modifiers allow readings where only the resulting state of a change-of-state verb holds for the stated amount of time as in (28) (though the acceptability can vary based on semantic factors having to do with

10 There are alternative analyses for again that are not scopal in nature, but instead rely in various ways on assuming that again attaches high (e.g. over the entire event structure of the surface verb, assuming there even is an event structure) and relying on a variety of purely truth conditionally defined means of somehow isolating the result state (see Jäger and Blutner 2003; Deo et al. 2013; Pedersen 2014; Beck and Gergel 2015; inter alia). While these analyses have some merits, we ultimately do believe that a scopal analysis of again better conforms to data we discuss below (see $\$ 2.4 .1$ ), plus also generally fits well with what we see as the broader justifications for event structures discussed in this chapter (see $\$ 1.4 .3$ and also $\$ 2.4 .2$, fn. 27). 
the nature of the state, such as how reversible it is; cf. the oddity of \#John killed Bill for three weeks, from Dowty 1979: 255):

(28) Sandy opened the windows for a few hours.

Here it is only the open state of the window that lasted for a few hours, not any of the opening events. Event structural approaches provide a natural account of this as scope over the root to the exclusion of any higher templatic structure, just as with restitutive again (see $\$ 1.6 .1 .1$ and $\$ 1.6 .2 .2$ for possible higher attachments for for an hour modifiers and for more on the semantic analysis of such modifiers):

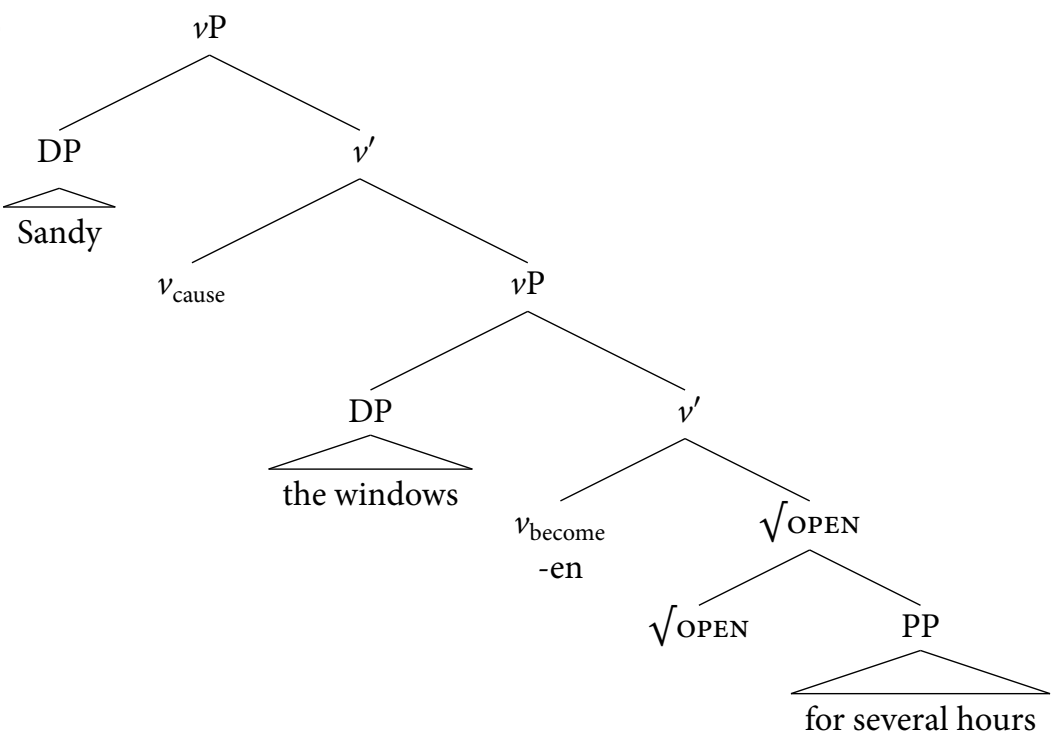

In sum, this approach makes predictions about the accessibility to semantic operations of different subparts of the verb's event structure, itself motivated by paradigmatic facts such as the entailment relationships between certain forms of the verbs such as the one between the causative and the inchoative. It is hard to see how a thematic role approach could capture such generalizations, suggesting the overall correctness of the event structural approach. The degree to which thematic roles are still a part of a linguistically significant semantic ontology, on the approach outlined here they can be derived from event structures as the sets of lexical entailments that must hold of the various arguments within an event structure. In this case, however, they are derivative notions rather than the sole basis for a theory of verb meaning.

An analogous treatment could be given for event structures implemented as lexical semantic objects as in $\$ 1.3 .1$ (per Dowty 1979). For example, if one were to assume lexicalized event structures then this level of representation would itself 
have to be subject to some set of interpretation rules. Provided the various pieces were given the same sorts of meanings we gave to the functional heads and roots here the same interpretations and interpretive facts would arise, though the syntax could be much less ramified. Such an approach can even capture sublexical modification without technically relying on phrase structural attachment ambiguities. As Dowty (1979: 264-9) demonstrates, an analysis of sublexical scope that relies on lexicalized event structures is possible, where flatten again would have different readings according to meaning postulates that derive that different parts of the lexicalized event structure of flatten are predicated of by some logical operator that introduces the semantics of repetition (see \$2.4.2).

The distinction between lexicalist and syntactified event structures is not a binary contrast, however. There can be approaches that represent admixtures of the two. For example, following in the mold of Kratzer (1996), who proposed to separate the external argument of the verb as being introduced by a separate head (which she labeled Voice but which is equivalent to the $v_{\text {cause }}$ discussed above, though see Pylkkänen 2008: 86-100 on distinguishing the two), it could be that for a change-of-state verb the argument of $v_{\text {cause }}$ is a VP representing change-ofstate into some lexically specified state:

(30) a. The rug flattened.

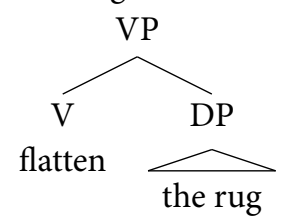

b. Mary flattened the rug.

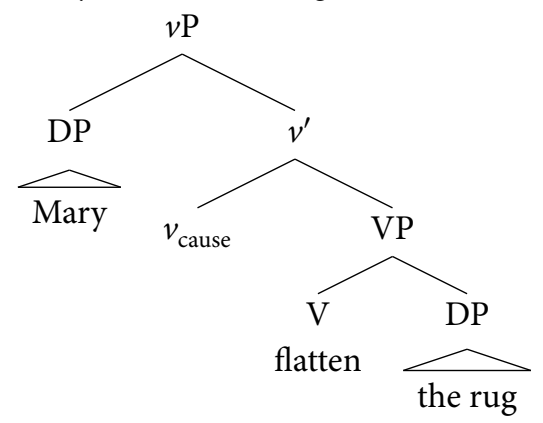

However, treating flatten as not decomposed will not capture sublexical modification facts such as the again facts. Thus we need to give the semantics of V itself as a translation into an inchoative event structure akin to (20b), and utilize some Dowty-style machinery to get restitutive readings. In this case the idiosyncratic root in the lexical semantic sense-the locus of idiosyncratic, non-templatic semantic information-does not line up with the idiosyncratic morphological 
root, here the V flatten. Rather, the lexical semantic root is a part of the morphological root's semantic representation, "flat" in (20b), despite the assumption of an at least partly syntactic event template.

Similarly, Fodor (1970) presented an extended argument that a verb like kill cannot be derived from cause to die since, among other things, kill represents direct causation rather than the indirect causation of cause (see \$1.6.2.1). However, the claim that kill entails direct causation is not a claim about root meaning in the sense we mean here. Rather, it is just a claim that the event structure for kill is not literally cause to die (see Harley 2012). But it could still have an event structure-needed to capture the various facts above-albeit one whose causal templatic element (e.g. $v_{\text {cause }}$ or CAUSE) has a meaning that is not the meaning of the English verb cause (see Dowty 1979: 96-8 for a more explicit discussion of the relation between templatic causal operators and cause). On this analysis again the surface morphological root kill is not the lexical semantic root, but represents an underlying causative event structure of one of the sorts above that contains a more specific lexical semantic root. Fodor's observations thus pertain to aspects of the event template, and not the lexical semantic root. In all that follows our focus is on the lexical semantic root regardless of whether it is a morphological root or not. When we use the term "root" it is the lexical semantic root we refer to, unless otherwise specified.

There are of course still other implementational possibilities. For example, in the style of event structures adopted by Pinker (1989) and Jackendoff (1990b) no consistently explicit, recurring ontological distinction is made between root and template in the way it has been made for the approaches outlined above vis-a-vis clearly delineated theoretical components corresponding to each. Rather, verbs are lexically associated with event structures (or semantic structures that have event structures as a component) and verbs that fall in the same class have shared aspects of their event structures but also points of variation on a verb by verb basis. However, the representations are still structured in a decompositional way and a root vs. template distinction arises as an emergent generalization over what is shared across verbs' event structures vs. what is unique to those of individual verbs. Grammatical correspondence principles such as argument realizational principles can be stated on the recurring event structural elements.

Thus provided that (a) decompositional structure is represented somehow, (b) there is a distinction between shared and idiosyncratic aspects of a given verb's event structure, and (c) grammatical and semantic principles of argument realization are derived from the templatic elements, then lexicalist, partly lexicalist, or fully syntactic formalizations of the idea of event structures of all stripes could in principle capture many if not all of the same things. For purposes of this study we will assume fully syntactic event structural representations with acategorial morphological roots such as those in (21) corresponding to lexical semantic roots, though the choice of implementation is largely immaterial (as we discuss further in 
$\$ 2.4$ in particular), provided the appropriate semantic distinction between event templates and roots is maintained and their relationship to the syntax is made explicit.

\subsubsection{Summary: The core properties of event structures}

The key point for any event structural approach is the assumption that a verb's meaning is partly represented by a decomposed event structure built from an event template that defines the event's broad temporal and causal contours and which may be shared across verbs, and an idiosyncratic, verb-specific root that fills in the real world details of some aspects of the event structure such as particular states and actions. This dichotomy is a key feature of all event structural approaches regardless of implementation. An assumption that also cuts across implementations is that the template and not the root is what largely determines a verb's grammatical properties such as its argument realizational or regular morphological properties. In more syntactified approaches it follows from the conflation of event structure and phrase structure wherein the templatic functional heads are the things that take arguments and introduce regular morphology, among other things. In lexicalized approaches the connection to syntax and morphology is through linking and correspondence rules, but again such rules are defined over properties of event templates, deriving essentially the same predictions (see e.g. Levin and Rappaport Hovav 1995: 135-66). Since the semantic structure that grammatical properties such as argument realization are defined in terms of is an independently motivated notion of subevental structure, these approaches provide a more motivated theory of a verb's grammatical behavior than one based on thematic roles and thematic role hierarchies (although there are still issues with decompositional approaches as per fn. 4; see Beavers 2010: 825-31).

Conversely, on most approaches the lexical semantic root is grammatically less significant, with theories differing in how much grammatical significance a root has. In some approaches (e.g. Borer 2005, 2013) the root has little role in the syntax at all. In other approaches the root's syntactic role might be to place constraints on what templates it can occur with. For example, Rappaport Hovav and Levin (1998: 109) associate roots with particular templates through a series of canonical realization rules where roots of a certain type are prototypically associated with certain templates (with the possibility of further template augmentation as per Rappaport Hovav and Levin 1998: 108-9). On more syntactified approaches, such as Marantz (1997: 217, (15)) or Alexiadou et al. (2006: 202, (51)) (see also Megerdoomian 2002: 184-204) roots may fall into intuitively-based semantic classes (akin to the verb classes defined in Levin and Rappaport Hovav 1995: 79-133, e.g. externally caused change of state vs. internally caused change of state) which are again associated with particular templates. Alternatively, Ramchand (2008: 12-15, 57-62) 
posits that roots come with formal features that indicate which templatic operators qua syntactic heads they must $(\mathrm{Re})$ Merge with in ways that ensure certain roots must occur within templates with certain heads (though additional augmentation may again be possible).

Crucial, though, is that the degree to which a root has an effect on the grammatical properties of the surface verb on such approaches it is indirect, in that the root just determines which template it occurs in. But it is still the template that determines the grammatical properties of the surface verb in terms of which arguments it may occur with and what the regular derivational morphology is as above (though of course roots conspire with templates in terms of determining irregular idiosyncratic and derivational morphology). An even stronger proposal comes from Kelly (2013), who associates roots with syntactico-semantic features borrowed concepts proposed in Hopper and Thompson (1980) and Dowty (1991) that place more direct conditions on the verb's surface syntax such as how many participants there must be in ways that constrain the templates. Thus there is variation across theories in how roots are related to the verb's grammatical properties. But a common assumption is that it is ultimately the template that bears the bulk of the direct work in determining the grammatical properties of the surface verb. Perhaps the only truly universally accepted direct grammatical role of the root is that it somehow correlates with the surface verb's idiosyncratic morphological root, either because it is the morphological root on fully syntactified approaches to event structures or because it is the one thing that distinguishes the otherwise identical event structures of distinct verbs on more lexicalist approaches.

\subsection{Hypotheses about root meaning}

Returning to the central theme for this book, we are now in a position to consider hypotheses about what constraints may exist on what the parts of an event structure mean that have predictive consequences. In particular, we are interested in the question of what defines the semantic contribution of the event template vs. the root for any specific event structure, e.g. what meanings $v_{\text {become }}$ and $v_{\text {cause }}$ vs.

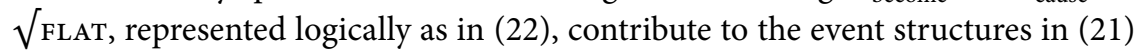
and how they are put together compositionally to form the meaning of a given sense of a surface verb. By focusing on the meanings of templatic heads and roots in a given event structure we are thus not necessarily making any claims about what any of them mean in any abstract sense divorced from context. Templatic heads and roots could, for example, be polysemous (or alternatively a single surface morpheme could underlyingly represent distinct heads or roots), as can be seen transparently in the context of Ulwa verb class morphology discussed by KoontzGarboden (2009b: 500-6). Furthermore, roots in some theories are assumed to not be associated with normal truth conditional content at all, but instead represent 
abstract concepts or bundles of concepts that only take on anything like a truth conditional meaning within a particular event structure. Thus we focus on the contributions of templatic heads and roots in particular event structures, by focusing on single senses of the surface words they correspond to.

For templatic heads the universal consensus is as above, namely that their meanings are basic, universal non-logical constants such as cause $e^{\prime}$ and become' defining broad temporal and causal eventive properties. But with roots the overwhelming view is that they introduce idiosyncratic content that somehow fills in specific event details. However, there have been few explicit proposals about what sorts of lexical entailments these can give rise to among the set of lexical entailments associated with an entire event structure. The degree to which this is true it is a significant lacuna in the study of event structural meaning. As Dowty (1979: 125-6) makes very clear, if a goal of an event structural approach is to make testable predictions about possible and impossible verb meanings, no matter how confident we are about templatic meanings if there are no constraints on the root meanings such that roots can mean anything then so can the verbs they give rise to. Fleshing out a theory of root meaning is thus important in fully realizing the predictive power of event structural approaches. (See Williams 2015: 216-8 for a recent reiteration of exactly this point and the need for work on this topic.)

One difficulty in studying root meaning is deducing what the event template and the root for a given verb or set of verbs (or other such word categories) are and then finding out what the contribution of each element is. Fortunately, the logic of event structural approaches gives us ways to probe root meanings. One way to hone in on root meaning is through the use of sublexical modifiers such as again in $\$ 1.3 .2$, which can be used in some cases to isolate a root in a given event structure. Furthermore, paradigmatic relationships between surface forms can give us a clue as to root meaning. By default, if we can identify a specific verb class with a welldelineated set of grammatical and semantic properties then we can take what is common semantically across all of the verbs in this class to reflect the contribution of the event template. Any other meaning in any specific verb must therefore be contributed by the root. Of course, when looking across morphological roots in a template there is the possibility mentioned above of templatic polysemy. To control for this the case studies we address in this book-(caused) change-of-state verbs and their associated adjectival forms plus ditransitive verbs of caused possession in the dative alternation - are such that it is commonly assumed that a single template or set of templates unites each one, so we generally set this possibility aside unless otherwise noted.

Additionally, by default, if we can identify a single morphological root and look at its uses across a range of distinct grammatical contexts assumed to correspond to distinct templates, any meaning common across all such usages must come from the root, factoring out known templatic meaning and provided other roots in those contexts do not give rise to this meaning. Of course, when looking across different 
templates at the same morphological roots there is the possibility mentioned above of lexical semantic root polysemy. However, we generally avoid positing such polysemy unless there is very good reason to, e.g. a meaning difference for a single morphological root across templates that seems difficult or impossible to capture assuming a shared root meaning. For most such comparisons we are interested in (change-of-state verbs and their related adjectives, transitive and ditransitive verbs across argument alternations that reflect distinct templates) there is rarely any reason to assume this. ${ }^{11} \mathrm{~A}$ combination of these three methodologies will give us the tools we need to ascertain what a root contributes to a given verb's meaning, and we can thus begin to identify possible constraints on root meaning.

This does all assume that a root actually has an isolatable meaning, and we do indeed make the straightforward default assumption that in event structures such as the ones we have in (21) each terminal has a meaning such as those discussed in $\$ 1.3 .2$ and that the meaning of the full structure is derived compositionally. This is not meant, though, to preclude that in the case of surface lexical idiosyncrasy or idioms (especially phrasal idioms like kick the bucket) that a unit larger than a terminal node may have an idiosyncratic, non-compositional meaning. But we assume that the default, regular case is compositional meaning, and in that case roots are assigned meanings themselves and the interpretation of the entire event structure is fully compositional. That said, even on approaches that do not make this assumption the kinds of questions we wish to address about what a possible root meaning is still arise. For example, Borer (2013: 403-17, 436-70) argues that roots are contentless phonological indices, and content only arises for a root plus some additional grammatical context $\mathrm{X}$, minimally a root plus a category label it takes on in context. But optionally a (categorized) root plus additional heads it has Merged with can instead take on a single non-compositional meaning as a unit (up to the point of the introduction of an extended projection, e.g. the introduction of arguments). However, for a given grammatical context $\mathrm{X}$, barring lexical idiosyncrasy or idiomaticity, for all roots in $\mathrm{X}$ the standard assumption is that there should be some shared meaning between them that correlates with $\mathrm{X}$, and the residue left by ignoring that meaning correlates with the root. In this case we can still ask questions about what kinds of meanings distinguish root $+\mathrm{X}$ combinations for different roots.

\footnotetext{
${ }^{11}$ An example of where we might posit polysemy is the case of the root of the verb hand that we discuss in Chapter 3, which we argue entails the templatic notions of motion and possession. But as Alec Marantz (p.c.) points out the nominal hand as in John raised his hand lacks any such entailments. However, given that the nominal hand requires none of what verbal hand requires and vice versa (e.g. it is not contradictory to say John handed Mary a towel with a pair of tongs) we assume that the two uses of hand are not synchronically derived from the same underlying root with a single denotation, though presumably there is an historical relationship between them. This position, and the facts tied to hand, are reminiscent of observations made in relation to locatum and location verbs by Kiparsky (1997: 485-91). In general, nominal forms of the roots we study here are an interesting potential source of further data, but for now we restrict our study mostly to verbs and some adjectives.
} 
To this point, there have been a few such hypotheses about possible constraints on the lexical entailments introduced by roots. We outline two here which will form the basis of discussion in the following chapters.

\subsubsection{The Bifuraction Thesis of Roots}

While it is widely (if not universally) accepted that templates and roots have distinct grammatical roles in the larger event structure, an underexplored question (though see Dowty 1979: 125-9, Goldberg 1995: 27-31, 59-66, and Wechsler $2005 \mathrm{~b}$, for related discussion) is whether there is an equivalent divide between the meanings that are introduced by roots and by templates. For example, are broad templatic notions like cause' and become' and the like only ever introduced templatically? Or can a root in some event structure have a translation that contains these sorts of non-logical constants as well?

The default assumption presumably is that if templates determine grammatical behavior semantically, such meanings should be excluded from the largely grammatically irrelevant roots (not least because the simplest assumption is that such meanings should only be introduced once). This is Embick's (2009: 1) "Bifurcation Thesis of Roots" and the assumption of Arad (2005: 79) (see also Borer 2005, 2013; Dunbar and Wellwood 2016):

(31) The Bifurcation Thesis of Roots: If a component of meaning is introduced by a semantic rule that applies to elements in combination [i.e. is introduced by a functional head- $\mathrm{B} / \mathrm{KG}$ ], then that component of meaning cannot be part of the meaning of a root.

To put it more informally, roots do not contribute the same broad temporal and causal semantics to a verb's meaning that templates do. If this hypothesis is correct, it makes very strong predictions about how systematic verb classes are semantically. Verbs with shared templatic meanings should in principle show identical morphological and syntactic properties (modulo lexical idiosyncrasy), because those shared meanings must come from shared templates and the templates are what defines the verb's grammatical properties.

Of course, Bifurcation need not hold. It could be that roots and templates introduce overlapping meanings. This is for example the assumption of (say) Goldberg (1995), who assumes (in a very distinct but intuitively analogous framework) that at least some idiosyncratic verb meanings are subtypes of constructional meanings qua templatic meaning (see e.g. p. 60), a conclusion we ourselves ultimately support. ${ }^{12}$ Similarly, Rappaport Hovav and Levin (1998: 109) associate

\footnotetext{
12 This assumes that in Goldberg's version of Construction Grammar constructions largely represent templatic meanings and verbs are more akin to roots, something suggested by many of her
} 
roots with templates in a way that relies on a semantic root classification that references templatic notions (see also Marantz 1997; Alexiadou et al. 2006). Many approaches, though, are silent on the issue. However, it is very much an open question what exact evidence and argumentation would truly decide the question of whether Bifurcation holds. A key goal of this book is to tackle this question head on, as a way of taking a first stab at constructing a theory of what limits there are on root meanings at all. Should Bifurcation prove incorrect, this would suggest that one of the tightest possible correlations of semantics to syntax class does not hold.

\subsubsection{Manner/Result Complementarity}

Bifurcation is a hypothesis about a ban on templatic meanings in roots, which are usually thought to be the home of more idiosyncratic meanings. Are there generalizations regarding what kinds of or how many idiosyncratic meanings a root can have? Asked purely as a question of the sheer number of truth conditionally distinct lexical entailments a root can have, it is unlikely there are any conditions. ${ }^{13}$ As Grimshaw (2005: 85) has proposed, a given idiosyncratic notion can be arbitrarily rich, e.g. states described by a root can have an arbitrarily large number of lexical entailments.

But we might instead ask whether there is a notion of too much idiosyncratic meaning in terms of how many types of distinct idiosyncratic information a single root can introduce based on some predefined ontology of types of meaning. So far, the logic of the system outlined above has assumed just two types of roots: those that describe manners and those that describe states (the former of which always modify $v_{\text {cause }}$ or $v_{\text {act }}$ and the latter always serve as arguments of $v_{\text {become }}$, though as emerges below nothing in particular guarantees this). Assuming these two basic functions for roots, for any given root describing an action or a state, what else can it introduce? Can a state-describing root also describe an action or

representations in (for example) pp. 48-64, though crucially in some cases verb meanings subsume constructional meaning. Not all Construction Grammar approaches adopt this assumption. For example, in his extensive work on the locative alternation Iwata (2005a,b, 2008) assumes that verb meanings themselves subsume the meanings of constructions, which are thus generalizations over verb meanings at various levels of granularity. In this case questions such as Bifurcation might best be examined solely within lexical meanings, which may include a component akin to a root (as Iwata 2008: 88 himself suggests) within a larger meaning that might be more templatic, essentially as per Dowty (1979).

${ }^{13}$ We say "truth conditionally distinct" lexical entailments since, as a reviewer points out, counting lexical entailments themselves is pointless. For any grammatical formative that lexically entails $P$ it also (trivially) entails $P \wedge \forall x[x=x]$, and of course $P$ is true iff $P \wedge \forall x[x=x]$ is true, meaning the two lexical entailments do not constituent distinct contributions to the formative's meaning. Properly speaking, we should group lexical entailments into equivalence classes of those that are truth conditionally nondistinct, and then count the equivalence classes, though we informally refer to this as counting truth conditionally distinct lexical entailments. 
can an action-describing root also describe a state? Are there limits on what kinds of idiosyncratic meanings a root can describe simultaneously?

A proposal first directly articulated in Rappaport Hovav and Levin (2010) (see also Levin and Rappaport Hovav 2013) suggests exactly this. They claim that a verb that describes a change of state never gives rise to manner entailments (e.g. an action) and a verb that describes a manner never gives rise to changeof-state entailments (see also Husband 2011; Mateu and Acedo-Matellán 2012). This follows from two conditions. A verbal root can either describe an action or a state but not both, and a surface verb's event structure can have just one root (presumably in the canonical position for its meaning):

(32) Manner/Result Complementarity of Roots: A verb can either have a root modifying a manner predicate and introducing only manner entailments, or root acting as the argument of a change predicate and introducing only state entailments, but not both.

Thus Manner/Result Complementarity as a descriptive fact about verb meanings rests on a theoretical claim about root meanings. If Manner/Result Complementarity holds, it makes quite striking predictions about possible verb meaningsnamely that they fall into two quite distinct classes-and that predicates that have both manner and result in them at once must arise only through composition of separate words into some larger construction (such as a resultative like sweep clean). To test the empirical claim, we need diagnostics for manner and result in a verb's meaning and then to check if any verbs ever entail both simultaneously. If so Manner/Result Complementarity is violated as a descriptive claim about verb meanings. Furthermore, if the manner and the result are both coming from the root and not the template-for which we need to utilize the methodologies above for identifying root meanings - then we know that roots can entail different kinds of idiosyncratic meaning simultaneously. This would in turn mean that roots can introduce meanings in places that are not canonically where those meanings are introduced. Should Manner/Result Complementarity prove incorrect, it would thus predict systematically wider classes of verb types than typically expected.

\subsubsection{The true distinction between roots and templates}

Testing Bifurcation and Manner/Result Complementarity requires detailed grammatical and semantic investigation of well-chosen verb classes. We claim, based on our two major case studies, that neither of these two hypotheses about what kinds of meaning a root can have are actually supported. Rather, we argue that nearly any meaning that has been ascribed to a templatic head can be found in some root meaning somewhere, arguing against Bifurcation. Similarly, based on several 
specific subclasses of our two broader case studies, we argue that Manner/Result Complementarity also does not obtain because the roots of certain classes of verbs entail both a manner and a resulting state at the same time (see also Goldberg 2010: 46-50; Rissman 2016; Beavers and Koontz-Garboden 2017a). Taken together, these results suggest that there are no strong limits on what kinds of and how much truth conditional content a root can have-it can entail broad templatic notions, and several types of idiosyncratic meanings all at once.

However, we are not suggesting there is nothing interesting to say about root meanings or their relationships to templatic meanings and ultimately to the verb's grammatical properties. Rather, as we develop arguments against Bifurcation and Manner/Result Complementarity, what emerges is that there is a significant distinction between root and template meanings and between the meanings of different types of roots, albeit the relevant distinctions are not ontological in nature but rather distinctions of specificity. In particular, there is a linguistically significant distinction between two types of lexical entailments found in verbs' event structures, one canonically introduced by templatic heads and one by roots. Templates introduce very general eventive lexical entailments about things like cause and change, while roots introduce more idiosyncratic lexical entailments that describe quite specific real world states and action. This accords with standard thinking, but there is an under-appreciated fact about this distinction we claim is key to why roots violate Bifurcation and Manner/Result Complementarity: lexical entailments may themselves be related to one another by entailment (see Beavers 2006, 2010). Crucially, root entailments, being the more specific type, may entail templatic entailments because the actions or states the root describes are defined in terms of the more general eventive or stative entailments. They may also entail other idiosyncratic meanings, if they are also part of their definitions as well.

For example, certain types of states are conceived of as being able to exist independently of a change that gave rise to them, but there are other types of states we may conceive of as only being the result of some change. Any root that describes such a state must also entail that change since it is part of the definition of, i.e. our understanding of, the state. Similarly, while some actions may be conceived of as not giving rise to changes, others may be conceived of as necessarily giving rise to changes by their very nature. A root that describes such an action will of necessity entail change. Thus from the natures of certain actions and states, violations of Bifurcation follow by necessity. Similarly, some states may require not just a change but a specific action to yield the change, and some actions may yield specific states. This will result in violations of Manner/Result Complementarity. Conversely, some states or actions may not be conceived of as requiring changes or specific actions or states respectively, but the roots that describe them nonetheless include such meanings anyway. We suggest this occurs when certain state/action plus change/causation combinations are conventional or frequent enough that a root (qua word) gets lexicalized for them. Thus violations of Bifurcation and 
Manner/Result Complementarity are not just attested but expected, a point we discuss at several points as our study unfolds and that we synthesize and develop more in $\$ 5.2$.

The ultimate picture is that many major types of templatic meanings are found in root meanings, and these can be largely crisscrossed with different types of idiosyncratic meaning. The result is a typology of roots rooted in what kinds of and how many templatic and idiosyncratic meanings they have, a typology that in fact mirrors common typologies of event templates (such as those in $\$ 1.3 .1$ ) and the kinds of specific instantiations of such event templates (such as resultative constructions). Thus, even though Bifurcation and Manner/Result Complementarity do not hold, it is still possible to develop a principled theory of root meanings, albeit one that arises from types of meanings they do entail rather than constraints on types of meanings they cannot.

The existence of templatic meanings in roots raises questions about the correlation of lexical semantics to a verb's grammatical properties. Do meanings such as these have any direct grammatical correlates? We show that templatic meaning in roots can determine aspects of the verb's grammatical behavior-including the verb's morphological make up and its argument realization possibilities-in ways similar to the fairly direct ways templates determine these behaviors, as opposed to the more indirect means of roots determining grammatical behavior discussed in $\$ 1.3 .3$. Furthermore, roots entailing manner and result simultaneously also have a wider range of ways of composing with the event template, being able to appear in either adjoining positions or as arguments, which has effects on the syntax of verbs with those roots in terms of their modificational properties, possibly among others. Thus roots can have their own direct effects on a verb's grammatical properties.

If roots entail the meanings of complex event structures and have direct grammatical correlates, is there any reason to even assume the root vs. template distinction? As we also show, although templatic meanings introduced by roots can have grammatical consequences they do not have the same types of grammatical consequences as the same meanings introduced by templates. Only templates introduce templatic meaning in the kind of structured, grammatically significant way that derives facts like sublexical modification and preferences for certain arguments to have a certain syntactic prominence as discussed above. For example, recapping a discussion in $\$ 1.3 .1$, in Mary flattened the rug the result state involves a series of conditions such that every part of the rug is flat. Yet in restitutive Mary flattened the rug again the modifier again must scope over the entire result, so that once again the entire rug is flat. Again cannot scope over just a subset of the root's lexical entailments, e.g. that again only half the rug is flat. Only templates provide the relevant structure to define multiple scopal attachment points. Likewise, we noted in $\$ 1.3 .1$ that postulating $v_{\text {cause }}$ in an event structure gives rise to a certain prominence relation between the agent and patient (modulo concerns raised in fn. 4). When roots introduce causal meaning this will not result in the same 
prominence relations. Thus despite our conclusions above, templates do still have a distinct role to play in the grammatical properties of verbs. ${ }^{14}$

In sum, we maintain that event structural approaches do still have predictive value. Roots and templates both fall into principled typologies regarding their meanings and systematic grammatical correlates, and combined with principles for how roots and templates are combined, the crisscrossing of those predictions still derives a typology of verb classes, which we discuss further as our studies unfold and return to the larger significance of in $\$ 5.4$. But given that there do not seem to be constraints on what roots can mean, following the logic of Dowty (1979: 125-6) there do not seem to be truth conditional constraints on what verbs can mean, at least as a consequence of anything about their roots. This in turn challenges the strong correlations of truth conditional content to event structures and thus ultimately to a verb's grammatical properties that hypotheses like Bifurcation and Manner/Result Complementarity would predict. Just looking at the truth conditions of a surface verb alone does not tell us anything about what event structure underlies its meaning and thus what its syntactic properties are. Rather, our results suggest that while a semantically-rooted typology of verb classes does exist it has not one but two sources of systematicity, with semantic and grammatical properties that can vary fairly independently, yet is no less predictive, once both factors are taken into account.

\subsubsection{Comparison to prior works}

As far as we are aware, few works have addressed root meaning in significant detail at the level of truth conditional granularity we aim for. Levinson (2007) (see also Henderson 2019 and Coon 2019) addresses root meaning as its central question and, building on Harley (2005), argues for four different semantic classes of roots: those that denote predicates of individuals, predicates of states, predicates of events, and functions from individuals to predicates of states. We adopt that assumption here as well, but the difference between our work and theirs is in the level of granularity-we show that there must be further distinctions within

\footnotetext{
${ }^{14}$ The idea that roots and templates introduce the same meanings but templates provide structure roots do not suggests that the fundamental crosscutting role of templates is essentially morphosyntactic: they provide grammatically relevant semantic structure. That they introduce certain sets of meanings such as causation and change is of course important, and a key part of the productive base of word formation. But in some cases this is a secondary function if the roots themselves also introduce those meanings. An open question is why templates would introduce those specific meanings as opposed to others, especially when they can be found in root meanings. Here we are less certain, though the types of meanings generally found with templates are fairly generic and applicable across a wide enough range of event types, making them fit naturally with roots that already entail them and make for presumably functionally useful augmentations for roots that do not. But whatever the reason, the way they do introduce these meanings is different from how roots do it, adding a type of morphosyntactic scaffolding roots lack.
} 
such classes, in that there are different types of stative roots and different types of eventive roots, and we furthermore clarify what sorts of meanings these roots can bring to bear in an event structure. Arad (2005) looks at the Hebrew root/template system (in the morphological sense of those terms specific to Semitic linguistics) and equates it to the root/template distinction of event structural approaches, while also developing an extensive medium-grain classification of roots in terms of real world actions, individuals, and states described. However, she does not provide a compositional analysis, and also explicitly assumes (but does not argue strongly for) something like the Bifurcation Thesis that we argue against (p. 79). Borer $(2005,2013)$ has laid out a particularly extended and strong view of lexical roots as being devoid of categorial features and content. However, neither work has addressed the specific questions we have outlined here, namely what kinds of lexical entailments roots can have in particular event structures-or at least what kinds they correlate with if one assumes, as with Borer (2013), that they have no necessary independent content-and how they classify roots into grammatically significant subtypes.

Other works have focused less on roots and more on templates-often building on the foundational work of Dowty (1979) — but have discussed root meaning to some greater degree. Pylkkänen (2008) provides a compositional semantics for roots and event templates that assumes (without comment) that both can assign thematic roles to the same arguments, something that will be part of our analysis as well. Ramchand (2008: 60-2) compositionally treats roots as predicates over eventualities that classify the states and events introduced by templatic heads, furthermore assuming that a single root can Merge with and thus classify the eventualities of multiple functional heads. Kelly (2013: 104-26) addresses root meanings and their relation to syntax in somewhat more detail. Compositionally, like Ramchand, he treats root denotations solely as predicates over eventualities, but also assumes they have syntactico-semantic features borrowed from Hopper and Thompson (1980) and Dowty (1991) that are not compositional in nature but that figure into principles of building event structures that predict certain mostly syntactic facts about argument alternations (see Marantz 1997: 217, Megerdoomian 2002: 184-204, and Alexiadou et al. 2006: 202 for DM proposals in some ways similar in spirit to Kelly's). In nearly all of these prior works the incorporation of the root into the template is largely based on syntactic features or intuitionistic semantic classes of roots, with few details of the exact truth conditional content introduced by the root discussed in a way that explains how the entirety of a verb's lexical entailments are derived from the combination of that template and that root in a compositional fashion (see also Goldberg 1995; Marantz 1997; Rappaport Hovav and Levin 1998). Our approach is to assume that if a root introduces a meaning that for all intents and purposes is exactly like that introduced by a templatic head, it must do so in the same way as that head, i.e. as part of its lexical semantic content that is incorporated into the larger event 
structure in a compositional fashion. From this it is possible to explain a range of morphological and syntactic facts exclusively from the lexical entailments of roots, rather than from features outside of the compositional system.

Thus our study addresses questions of what types of truth conditions roots introduce into event structures, something not addressed in prior works. Our starting point is Bifurcation and Manner/Result Complementarity, but the degree to which refuting these two hypotheses is significant will, as noted, depend on one's theoretical assumptions. For proponents of these hypotheses the analyses we present will represent challenges, but for those who do not accept these hypotheses (explicitly or who have remained agnostic), the results may seem unsurprising, if welcome. However, a core contribution is in detailing the evidence that fully decides these issues, which requires looking carefully at root truth conditional content. In doing so we therefore more fundamentally begin to address the basic lacuna in event structural approaches first detailed by Dowty (1979: 125-6) in illuminating what roots can mean, so as to address what truth conditional predictions about surface verbs event structural theories make to begin with, and the consequences they have for theories of semantic verb classes and their grammatical properties.

\subsection{Outline of the study}

The structure of the following chapters primarily follows different ways that the Bifurcation Thesis could be invalidated for standard, uncontroversial templatic semantic notions. Along the way, we turn to the question of Manner/Result Complementarity and the larger typology of roots that it suggests, crisscrossing the results we obtain from examining templatic meaning in roots with roots that have both manner and result in them to produce a larger typology of possible roots rooted in how templatic notions and the notions of manner and result can be combined.

Chapter 2 examines the semantic and morphological predictions of Bifurcation, focusing on the roots of change-of-state verbs and their stative correlates, the case study that Embick (2009) focused on initially. We show that there are two distinct classes of roots-roots from which descriptively deadjectival verbs are derived in English (the flat class) and roots from which descriptively monomorphemic verbs are derived (the crack class). Contradiction tests, sublexical modification, and evidence from morphological typology show that while the former class behaves in ways that are consistent with Bifurcation, the latter does not. Instead the roots of the crack class themselves seem to carry an entailment of change of state, i.e. the meaning normally found in $v_{\text {become }}$ which is otherwise a templatic entailment. We develop a formal analysis of this distinction that explains the different behavior of the two root classes but requires us to abandon Bifurcation, and also to admit 
that the truth conditional content of roots can have an impact on the regular morphological form of surface verbs and adjectives based on those roots. We also consider a variety of alternative analyses that preserve Bifurcation, and suggest that they come at some theoretical and empirical cost, and that an analysis that rejects Bifurcation altogether is preferred.

Chapter 3 extends Bifurcation to the novel case study of English ditransitive verbs of caused possession, which exhibit two argument frames that reflect their association with two distinct event templates, typically thought to represent caused motion in the to frame and caused possession in the indirect object frame. We show that these two templates are in fact highly underspecified semantically (recapping Rappaport Hovav and Levin 2008 and Beavers 2011a), and it is instead the roots that occur in a given template that fleshes out many of the basic eventive entailments of the surface verb. These semantic details include change of state again, plus also possession and co-location, the very two notions that are otherwise uncontroversially considered part of the templatic structure of ditransitives and other verbs, arguing again against Bifurcation. We furthermore show that in some cases this truth conditional content can influence whether or not a given root regularly shows the dative alternation, a root-conditioned syntactic effect. We develop an analysis of root/template composition that crucially relies on manner-adjoined roots being able to impose conditions on the template-defined result states in a way that sometimes allows roots to augment the templatic meaning and sometimes vice versa. We again consider counterproposals that might retain Bifurcation, and suggest that they are dispreferred for various reasons.

Chapter 4 turns to the question of what limits may exist on how many types of idiosyncratic meanings a root can entail, and the case study here comes from Manner/Result Complementarity. We propose that there are systematic verb classes that entail both meanings at once. To build the case for this claim we first isolate appropriate diagnostics for manner and result entailments as part of the meaning of a surface verb and show that certain surface verbs do entail both at once. Then, on the basis of sublexical modification, we also argue that when any single surface verb entails both of these at once they must be part of the meaning of a single event structural root and not two separate roots (i.e. that manner+result verbs are not somehow akin to resultative constructions that entail manner and result at the same time through an explicit compositional process). In other words, no event structure can have both a manner root and a state-denoting root at the same time, supporting Manner/Result Complementarity as a condition on how many and what types of roots may occur in a single event structure, even though this does not rule out verbs that nonetheless do entail both. The conclusion then is that roots can have manner and (result) state entailments in them at the same time, and thus there do not seem to be obvious constraints on how many types of idiosyncratic meanings they can entail. We then conclude by considering the presence of causation (i.e. the semantics typically ascribed to $v_{\text {cause }}$ ) in the 
meanings of the same roots, ultimately arguing once again against Bifurcation for yet another templatic meaning.

Chapter 5 summarizes our results and considers other constraints on possible root meanings that we also suggest may not hold. We outline the larger typology of root types Manner/Result Complementarity-violating and Bifurcation-violating roots give rise to. We then consider the deeper motivation of why roots would not be subject to constraints such as Bifurcation and the conditions required for Manner/Result Complementarity. We outline in more detail a theory of templatic and root lexical entailments and how entailment relationships may hold between them and why, which we suggest provide the clearest motivations for violations of these two constraints, while also maintaining that the root vs. template distinction is still necessary for a theory of verb meaning. Before we turn to our primary case studies, we offer a few additional preliminary assumptions concerning the meanings of change and causation that will be relevant in the following chapters.

\subsection{Background assumptions: Defining causation and change}

\subsubsection{Change of state as scalar change}

The notions of causation and change (and the states that change is based on) are unquestionably the most prominently studied templatic meaning components, and are so basic to event structural approaches that they will appear in every chapter here. Above we kept the discussion of the meaning of terms describing states, change of state, and causation fairly informal, and as it happens, for most of the study we present here fairly simple, informal definitions of these notions will suffice for us to make our central claims. Nonetheless, in a few instances we will need to rely on more precise characterizations of these notions, especially change of state. For this reason it is appropriate to discuss in more detail their truth conditions. We are largely relying on extensive prior work and what we outline here is only a sketch to synthesize and clarify some of the main insights of what we import from these prior works-see the references cited throughout for more rigorous treatments. We first consider states and change of state, which will be particular interest to us, before turning to causation.

\subsubsection{Aspectual motivations}

The first question is what the meaning of change of state is here, i.e. the truth conditions of become'. A common understanding is what we informally assumed above, namely that become' relates an individual to a state and an event and says that at the end of the event the state holds of the individual and prior to that point in the event the state did not hold (building on Dowty 1979: 140, (11), albeit introducing events whereas Dowty dealt with temporal intervals). For much of our discussion, this simple notion would be sufficient: 
(33) For all $s, e$, become $(s, e)$ is true iff at the beginning of $e$ the state $s$ does not hold and at the end of $e$ the state $s$ does hold.

This understanding of the meaning of become' is common in works on event structure (so much so that it is often not even made explicit). Crucially, $s$ is treated as some eventuality that must occur and will have certain properties that hold of it (e.g. $P$ in (22b)), sufficient to account for the common diagnostic of change of state as yielding contradictions when asserting that the change occurred but that the relevant state has not obtained, as in (6) in $\$ 1.1$.

However, it is has become common in much work on lexical semantics to assume that states and thus changes of state are instead scalar (see e.g. Tenny 1987, 1992, 1994, Dowty 1991, Jackendoff 1996, Krifka 1998, Filip 1999, Hay et al. 1999, Kennedy and Levin 2008, Rappaport Hovav 2008, Beavers 2011a,b, 2012, and Beavers and Koontz-Garboden 2012, among numerous others, for various views along these lines, though the term "scale" is not always used in all of them even if there is some aspect of the analysis that is equivalent to a scale as described here). On this approach, properties may hold to different degrees ordered along a scale of all possible degrees to which the given property can hold. A state is thus defined in terms of a triple $<\delta, S, R>$ :

(34) a. $\delta=$ some property/dimension (e.g. for height, length, straightness, temperature, proximity to some reference point)

b. $S=$ a set of (intervals of) degrees for having property $\delta$

c. $R=$ an ordering of members of $S$ (determining directionality)

Adjectives and other stative terms assert that a given individual holds a certain degree of the property, e.g. The rod is straight entails that the rod holds a degree $d \in S$ of $\delta$ =straightness, in this case by default the maximal possible degree according to $R$ (i.e. completely straight). Change of state involves coming to be in a state of holding some specific $d \in S$ of $\delta$ greater than the degree held at the beginning of the event according to $R$. For example, The rod straightened entails there is an event $e$ at the beginning of which the rod held degree $d^{\prime} \in S$ of straightness and at the end it held a higher degree $d \in S$ of straightness according to $R$ (by default the maximal degree).

Evidence for a scalar analysis of change comes from aspectual properties of change predicates that can be neatly modeled in this approach. One is telicity, i.e. whether the predicate entails that the event terminates at a particular point. A standard telicity test is the in an hour vs. for an hour test of Dowty (1979: 56-8), the former preferred with telic predicates and the latter with atelic predicates. Shatter a vase patterns as telic and lengthen his pants as atelic: ${ }^{15}$

\footnotetext{
15 The intended reading of for an hour here is that the event went on for an hour, not the reading discussed in $\$ 1.3 .2$ where the pants were long for an hour (before they were shortened again). Owing
} 
(35) a. Sandy shattered a vase in/?for an hour.

b. Sandy lengthened his pants for/?in an hour.

More specifically, we assume a predicate is telic iff for any event in its denotation no non-final subevent of that event is also in its denotation (see Beavers 2012: 34-5, in an update to Krifka 1998: 207, (37)). For any event in shatter a vase the last point in the event is when the vase is shattered; any subevents of such an event that lack that final point will lack that shattered state and thus not be in the denotation of shatter a vase. Conversely, lengthen the pants events can have non-final subevents also in lengthen the pants, e.g. when lengthening the pants four inches is part of lengthening them five inches, both of which are events in lengthen the pants, and thus the predicate is atelic.

Crucially, some verbs derive predicates of change that can be telic or atelic contingent on the interpretation of certain event participants we refer to here as incremental arguments for reasons that will become obvious below. For example, a longstanding generalization is that the interpretation of the patient can figure into a predicate's telicity (Garey 1957; Verkuyl 1972, 1993; Dowty 1979, 1991; Tenny 1987, 1992, 1994; Krifka 1989, 1992, 1998; Jackendoff 1996; Filip 1999; Beavers 2008, 2012; inter alia). Building on Krifka (1998: 209-22) in particular, if the patient of drink is expressed as aP that has quantized reference (i.e. for any entity it describes it describes no subpart of that entity, as with definite singular count DPs) the predicate will be telic. Otherwise it will be atelic. Thus a glass of wine has quantized reference (no subpart of a glass of wine is also a glass of wine) and drink a glass of wine is telic, whereas wine has non-quantized reference (i.e. subparts of wine can still be wine) and drink wine is atelic:

(36) a. Sandy drank the glass of wine in/?for an hour.

b. Sandy drank wine for/??in an hour.

The key intuition (see e.g. Tenny 1987, 1992, 1994; Krifka 1989, 1992, 1998; Dowty 1991) is that the verb entails a homomorphic relationship between the event's and the patient's mereological breakdowns, where each unique part of the event corresponds to a unique part of the patient and vice versa (Krifka's 1998: 213, (51) Strictly Incremental Relation). Thus as an event in drink a glass of wine progresses at each unique part of the event a unique part of the glass of wine is consumed, and the final part of the event will correspond to the last part of the glass of wine. But any non-final subevent of such an event will not be related to that final part of

to the possibility of various types of aspectual coercion (with a certain amount of contextual support; see e.g. Krifka 1998: 215; de Swart 1998: 361-2, and; Rothstein 2004: 114-5, 190-1, inter alia), for PPs are sometimes possible with telic predicates and in PPs with atelic predicates. But the primary generalization is that each is more natural out of the blue with only one type of predicate. 
the glass of wine and thus will not be an event in drink the glass of wine. Rather, it will be an event of drinking less than the full glass of wine. Conversely, in at least some events in drink wine there is a non-final subevent of drinking a subpart of the wine drunk. But this subpart is still itself wine and thus this subevent still falls under drink wine. ${ }^{16}$

Returning to scales, sometimes telicity depends on whether the final state on the scale is entailed to be some specific state by the verb or some additional dependent such as a PP or AdjP in a resultative construction (i.e. there is "quantized change" as per Hay et al. 1999: 132-8 and Beavers 2011b: 358, (60)). Thus in (37a) the predicate describes the final state (where the scale is defined by positions along the path), and the predicate is telic, but in (37b) the final state is not described and the predicate is atelic: ${ }^{17}$

a. Sandy walked to the store in an hour/?for an hour.

b. Sandy walked for an hour/??in an hour.

Krifka (1998: 225, (71)) analyzes such changes via another homormorphic relation, a Movement Relation, between the event and the path starting at the patient's initial position and ending at its final position. Movement Relations preserve part-whole structure plus adjacency, where temporally adjacent progress through the event corresponds to spatially adjacent progress along the path. On this view, any event in the denotation of walk to the store will have as a final point the patient reaching the store, and any non-final subevent will be an event of stopping short of the store and thus will not be in walk to the store. But some events in the bare predicate walk have other walking events as a subpart. In some cases of walking along a path there is a non-final subevent that is also walking along some path, namely a non-final subpath of the larger path. Thus the predicate is atelic. In sum, the interpretation

16 There are certain exceptions to this generalization, e.g. in Sandy ate more than three slices of pie in/?for an hour the object lacks quantized reference but the predicate is still telic. Solutions to this that preserve the homomorphic mapping insight typically involve introducing additional operators that maximalize the interpretation of the theme or event to the biggest such theme or event in context which (roughly) has the effect of ensuring quantized reference for the relevant incremental argument XP and/or event description. We set this aside here, but see Zucchi and White (2001), Filip (2004), Filip (2008), and Kardos (2012) on maximalization (and see Rothstein 2004: 152-4 for a critique and Beavers 2012: 44-7 for a response).

${ }^{17}$ Motion along a path is often defined as a type of scalar change, either by assuming that the path is the scale in the sense that the ordered positions along the path are equivalent to ordered degrees on a scale and function in the same way (Krifka 1998: 228-30; Beavers 2012: 52-8; Zwarts 2006: 18-20, 2008: 98-103) or that the degrees on the scale measure the relative distance from the source or to the goal along the path without conflating the the scale and the path (Hay et al. 1999: 139-40; Tatevosov 2012). Indeed, on the analysis of Krifka and Beavers in particular all scales are defined in terms of a notion of "path" that can be physical or abstract, and scalar change is thus analyzed as (metaphorical) motion along a path. The exact implementation here is largely irrelevant, and we will assume some correspondence of a path and a scale and in the case of motion predicates informally treat them as interchangeable unless there is reason to make a distinction (as we will do in our discussion of motion readings of ditransitives in $\$ 3.5$ ). 
of patients and scales figures into the calculation of telicity via homomorphic relationships to the event, subsumed under one mereological definition of telicity.

More recently, Beavers (2012) demonstrates that both types of incremental arguments are relevant for the telicity of all predicates of scalar change. Consider (38a), where the patient has quantized reference and the final state is described by a goal PP, and the predicate is telic. But in (38b) there is quantized change but not quantized reference of the patient, in (38c) there is quantized reference but not quantized change, and in (38d) there is neither, and all three are atelic:

(38) a. The earthquake shook a book off the shelf in/?for a few seconds.

b. The earthquake shook books off the shelf for/??in a few seconds.

c. The earthquake shook a book for/??in a few seconds.

d. The earthquake shook books for/??in a few seconds.

(Beavers 2012: 25, (2.5))

Beavers argues that the relevant homomorphic relation involves a ternary relationship between the mereological properties of the event, the patient, and the scale that combines the two binary homomorphic relations discussed above by (a) decomposing the event isomorphically by parts of $x$ as per a Strictly Incremental Relation and (b) relating each such subevent to a part of the scale as per a Movement Relation. For example, in The soup warmed from 50 degrees to 100 degrees the soup is a mereologically complex object that has a temperature of 50 degrees at the initial point in the event. As the event progresses, the soup warms, though perhaps not evenly; some parts may warm faster than others. The progress may not be even; some parts may warm and also cool on their way from 50 to 100 degrees. But each change will be from one degree to an adjacent degree. The event culminates when all of the soup is 100 degrees. Informally, the relevant condition on incrementality is the following, whereby each subpart of the theme undergoes its own scalar change (translating the mereological model of Beavers to sets of degrees): ${ }^{18}$

(39) Figure/Path Relation: An event $e$, patient $x$, and continuous, ordered set of degrees $S$ on some dimension $\delta$ stand in a Figure/Path Relation (FPR) iff every unique part $x^{\prime} \leq x$ corresponds to a unique subevent $e^{\prime} \leq e$, the sum of all such subevents constitutes $e$, and each $e^{\prime}$ stands in a Movement Relation with a continuous subset $S^{\prime} \subseteq S$, where $S^{\prime}$ includes $x^{\prime \prime}$ 's initial degree of $\delta$ in $e^{\prime}$ and where the maximal degree in $S^{\prime}$ is $x^{\prime}$ s final degree of $\delta$ in $e^{\prime}$.

\footnotetext{
${ }^{18}$ Not reflected here, but which follows from the Movement Relation, is that for each degree a part of the patient is related to there is a state of that part of the patient holding that degree on the scale at that part of the event.
} 
This analysis captures the role of incremental arguments above. As Beavers (2012) demonstrates, as long as the quantity of the theme and the final degree on the scale are entailed to be something specific then that quantity of the theme reaching that degree on the scale will define the event's terminal point.

A second aspectual property is durativity. Punctual change predicates describe events that have just two moments, one before and one after the change. Durative predicates describe events with more parts (Beavers 2008: 254). One durativity test for telic predicates is the interpretation of in an hour modifiers (Kearns 2000: 206), which have only an after reading with punctual predicates but an after or during reading with durative predicates: ${ }^{19}$

(40) a. Sandy can shatter a vase in an hour.

(after an hour)

b. Sandy can straighten a rod in an hour.

(during/after an hour)

Durativity likewise depends on incremental arguments. Punctual predicates arise if the patient DP referent is interpreted as not having mereological complexity (it is interpreted as an indivisible atomic unit) and the scale is binary (non-gradable), i.e. it consists of just a contrast between two degrees (Beavers 2008: 250-4). Thus for cross in (41a), if the patient is interpreted as atomic and the path is a border defined by a contrast between two locations, the predicate is punctual. But if the patient or the path is interpreted as more complex, or both are, the predicate is durative, as in (41b-d): ${ }^{20}$

(41) a. The settler will cross the border in an hour.

(after an hour)

b. The settler will cross the desert in an hour.

(during/after an hour)

c. The settlers will cross the border in an hour. (during/after an hour)

d. The settlers will cross the desert in an hour. (during/after an hour)

(Beavers 2012: 49, (2.49))

As Beavers (2012: 47-52) discusses the FPR relates the part-whole structure of the theme and the scale to the event in such a way that an atomic theme and non-gradable scale will produce a two point event and thus the predicate will be

19 This test produces sharper results when there is an overt auxiliary, hence we include one when applying it. Atelic predicates are categorically durative, and for an hour applies. Note that the after interpretations do not require that nothing at all happens prior to the change. Rather, in many contexts it is possible that some process leading to the change is going on, e.g. Sandy struggling to lift a hammer in (40a) before she manages to hit the vase. But crucial here is that no particular action is required, and the predicate also allows readings in which literally nothing happened save the instantaneous change

${ }^{20}$ As Beavers (2012: 50-2) discusses, a certain amount of context is required here. Whether a theme is atomic or even whether a scale is gradable depends on how the relevant entities are interpreted. For now we assume a fairly neutral context that assumes a single settler is interpreted as atomic but multiple ones are not, and a border is a two point path but a desert is not, though there may be contexts where they could be interpreted otherwise. 
punctual (though see $\$ 1$ 6.2.2 for one caveat to this). Any additional complexity in the theme or the scale will result in a durative predicate over events with nonminimal mereological complexity.

Thus a scalar analysis coincides with a traditional, more purely temporal model of change in that some state obtains at the end of the event that did not obtain before. But it introduces an ordering of the degrees to which different states of holding a property correspond to upon which incremental progress can be defined that allows us to model aspectual properties of change-of-state predicates. In the simplest terms, we need to update (33) to include not just that the final state obtains but that it is a scalar state that arose through incremental transitions through other degrees ordered along the same scale according to an FPR, predicting the aspectual correlates of change. For our case studies the details of this are largely irrelevant, and simply assuming this informally is sufficient. Nonetheless, we outline here a sketch of a model of how to do this, though it is certainly incomplete and little will depend on the exact details. First, though, we discuss a key aspect of scalar change, namely the central notion of comparison of degrees. ${ }^{21}$

\subsubsection{Scales and comparison}

In gradable adjectival predicates, there is a role of context in determining what the state being described is in terms of the degree on the relevant scale, suggesting a contextual-interpreted variable of some sort. The usual understanding is that the patient holds the relevant property to a degree that is above some contextually defined degree known as the positive standard, whose interpretation depends on context and scale type (see Kennedy 2007 for overview and references). For adjectives that describe states on scales for which there is a unique maximal value (e.g. as with straight in (42a)) the positive standard is by default the maximal value. With scales that lack a unique maximal degree but for which there is a minimal degree (e.g. as with wet in (42b)) the positive standard is the minimal endpoint. With open scales, where there is no minimal or maximal degree (e.g. as with wide as in (42b)), the positive standard is derived from pragmatic context:
a. The rod is straight.
b. The towel is wet.
c. The road is wide.

(positive standard is scalar maximum)

(positive standard is scalar minimum)

(positive standard is from pragmatic context)

However, those are just the defaults. Even for scales with endpoints pragmatic context can define the standard if the context is rich enough. For example, The rod is straight can be true even if it is slightly curved provided there is a specific

\footnotetext{
${ }^{21}$ We wish to especially thank Itamar Francez (p.c.) for helpful discussion on these points.
} 
context where straight is defined as "the minimum level of straightness needed to run a certain type of scientific experiment on the rod".22

Conversely, for comparative adjectives the target argument must hold the property to a degree above the degree to which it is held by the stated comparison individual (if specified, otherwise the individual must be known from context):
a. The rod is straighter (than the other rod).
b. The towel is wetter (than the cloth).
c. The road is wider (than the driveway).

For superlatives (e.g. straightest, wettest, widest) the comparison is the highest of all items being compared. In neither case does scale type matter.

For change-of-state verbs, there are two relevant degrees - the degree to which the property holds at the beginning of the event and the degree to which it holds at the end (as per Hay et al. 1999; Kennedy and Levin 2008). There are also two conditions to be accounted for. The first is that the final degree must be higher than the initial degree: ${ }^{23}$
(44) a. \#He straightened the rod, but it's just as straight as/curvier than before.
b. \#He wetted the towel, but it's just as wet/drier than before.
c. \#They widened the road, but it's just as wide/narrower than before.

Second, the final degree depends at least partly on scale type, but in a way distinct from adjectives. As discussed in detail by Kennedy and Levin (2008: 168-70), with minimal or maximal endpoint scales as in $(45 \mathrm{a}, \mathrm{b})$ the final state is by default the endpoint, whereas for open scale adjectives the condition is that the patient have a higher degree than at the beginning of the event, as in (45c) (i.e. just that some change occurred):
a. He straightened the rod.
b. He wetted the towel.
c. They widened the road.

(final degree is scalar maximum)

(final degree is scalar minimum)

(final degree is above initial degree)

\footnotetext{
${ }^{22}$ Nonetheless, with maximal endpoint adjectives there is still a tendency for pragmatically defined standards to be close to the absolute maximum, e.g. it is difficult to find a context where a nearly perfectly round rod would be deemed straight. This might suggest that what counts as completely straight is instead vague, with context resolving some of the imprecision rather than assuming an absolute maximum which context may override, though we leave this aside here.

${ }^{23}$ A systematic exception to this discussed extensively in Beavers and Koontz-Garboden (2017a) are motion verbs where the patient undergoes a change but because of special properties of motion scales doubles back on its original position (e.g. Sandy circled back to his original position). However, this still requires the patient to have traveled a positive distance along the path, effectively meeting the condition that some change actually occur.
} 
Once again, those are just defaults. For maximal endpoint adjectives an alternative is to treat the initial degree as the standard, which in turn derives a telicity alternation (e.g. He straightened the rod in five minutes corresponds to a maximal standard, He straightened the rod for five minutes corresponds to the standard being the initial degree of the rod). Pragmatic context can also override scalar endpoints. Thus in a context where a rod needs to be straight enough for a specific scientific experiment to be conducted, but not necessarily perfectly straight, The scientists straightened the rod is still acceptable. Finally, pragmatics can supply a maximal value for open scale verbs, providing a telic reading, e.g. The coffee cooled in five minutes is fine in a context where we know coffee must cool to a certain (drinkable) degree. Of importance here is that the positive standard of the simple adjective is not relevant for verbs. Instead verbs have a different standard-the verbal standard-despite the verb seemingly being derived from that adjective, just as it is not relevant for comparatives and superlatives. Thus The road widened does not entail The road is wide, just that the road is wider than before. ${ }^{24}$ In sum, the category of the scalar term matters for what the final degree is.

Generalizing across all of these cases, there is always a condition that the (final) state is one of holding a degree of the relevant property that is at or above some standard determined by scale type, word category, and pragmatic context. In other words, none of these scalar predicates describe the having or coming about of just any degree of the relevant property, i.e. The road is wide(r) does not mean The road has the property of having width, and The road widened does not mean The road came to have the property of width that it did not have before. ${ }^{25}$ Rather, each predicate defines having or coming to have a degree defined in comparison to some other degree.

Returning to event structures, one way to account for the variable ways comparison is done in states and changes of state is to follow Kennedy and Levin (2008) and assume the root denotes a measure function that takes as its input an individual

\footnotetext{
24 This in turn means there is no semantic reason to assume that so-called "deadjectival" verbs are truly deadjectival in the sense of being based on a surface positive degree adjective form. Kennedy and Levin (2008: 170-80) do ultimately offer a unified analysis of the positive and verbal standards of change-of-state verbs by defining the scale upon which deadjectival verbs are based as always having a minimum value that is the initial state of the patient (the verbal scale being essentially derived from the adjectival scale by truncating values below that degree). This effectively creates minimal endpoints for the scales of all verbs of change that can serve as the standard, though maximal endpoint scales default to their usual maximal reading. We capture the same facts below in a different way, and leave for future work whether what we propose can be fully aligned with Kennedy and Levin's approach.

25 This claim should not be confused with the claim in Francez and Koontz-Garboden (2017: 38) that sentences like The road is wide are truth-conditionally equivalent (if sometimes differentiated pragmatically) to sentences like The road has width, the latter which has a standard just like the former. Our claim here is simply that The road is wide has truth-conditions that do have a minimum standard on the scale of width, and the same for the change-of-state counterpart. In other words, the use of a possessive construction above should not be construed as a claim about the truth-conditional relationship between an adjectival predication and a possessive one based on the same underlying gradable notion.
} 
(and a time, or alternatively a state as we assume here) and outputs the degree to which the individual has the relevant property, letting higher operators (e.g. functional heads) introduce comparison of that degree to the appropriate standard. (Or, to preserve the notion that roots ultimately derive truth values, that roots relate an individual to a degree and a state/time as per Cresswell 1977.) However, since comparison to some standard exists across all scalar expressions, an alternative would be to assume that the root itself has as a part of its meaning some underspecified standard that the (final) degree has to be above but whose value will be determined later by various linguistic and pragmatic contextual factors. There is in fact reason to assume this analysis over one that introduces comparison to a standard above the root, namely the interaction of scalar semantics with again modification.

As discussed in $\$ 1.3 .2$, again may apply to roots or to categories larger than a root. If it applies over the root, it asserts just that the relevant state held before. However, the prior state in all cases is crucially also one in which the degree to which the patient holds the relevant property is greater than the standard as determined by context and category, just as with the state that holds at reference time. Consider a simple adjective with again modification:

(46) The road is wide again.

Here the road must now be wide above the positive standard and also have previously been wide above the positive standard, e.g. whatever positive standard pragmatic context provides. The exact degrees of width need not be the same, however. The road could be wider now than it was before provided both degrees of width are above the standard. Crucially, if the comparison to the standard were introduced above again then a reading should arise where the road holds a degree of width above the standard now and before it held some degree of width that is not above the standard (if the two states are allowed to have different degrees), which is not the right result.

Similarly, consider restitutive readings with verbal predicates on a telic reading, requiring a contextual non-minimal standard:

(47) The workers widened the road again in five weeks.

Here both the final and earlier degrees of width must be above whatever the contextual standard is. The reading cannot be that the current widening brought the road above the standard and the prior width it held was below, nor is it that it had the same width each time. For example, consider a context where all roads must be $30^{\prime}$ wide, but this road was built with a width of $20^{\prime}$, was narrowed to $15^{\prime}$, and then the workers widened it to $30^{\prime}$. In this case (47) is not an acceptable description of that widening event. In sum, comparison to whatever the 
standard is must be introduced below the attachment point that derives restitutive modification, which we assume (following standard assumptions) is the root. Thus comparison to a standard is part of the meaning of the root, even if the details of what the standard is (e.g. the positive or verbal standard) is determined by the larger linguistic and pragmatic context.

\subsubsection{The truth conditions of scalar change}

A question that arises is how exactly to model this. Formally speaking scalar analyses have been implemented in several ways, e.g. with degrees based on points on a scale as per Cresswell (1977: 266, (2.1)), Hellan (1981: 42-4), and Pinkal (1989: 147-51), inter alia (as used informally above), or the interval-based approach of Kennedy (2001) and Schwarzschild and Wilkinson (2002), inter alia, or the mereological approach of Krifka (1998: 228-30), Beavers $(2008,2012)$ and Kardos $(2012,2016)$, inter alia that treats scales as a type of broader path object. Since our focus is on the coming about of states and since the formal details of the specific scalar analysis will ultimately play little role in our proposal, we represent the scales and the standard not as separate formal objects but instead as part of the truth conditions of the relevant logical predicates, using a simple degree-based approach in spelling out the truth conditions for expository convenience.

For a root like $\sqrt{\text { FLAT in }}(22 \mathrm{a})$ (repeated in (48a)) we define the truth conditions of flat' as in (48b), tying the state of flatness to some particular degree above some underspecified, patient- and scale-specific standard $\mathbf{d}_{x}^{\delta}$ :
a. $\llbracket \sqrt{ }$ FLAT $\rrbracket=\lambda x \lambda s\left[\right.$ flat $\left.^{\prime}(x, s)\right]$
b. for all $s, x$, flat ${ }^{\prime}(x, s)$ is true iff there is a degree $d \geq \mathbf{d}_{x}^{\text {fLATNESS }}$ and $s$ is the state of $x$ holding degree $d$ of FLATNESS.

Assuming the head that produces the adjective (which we label Asp following Embick 2004: 363; see \$2.2) just supplies a category and does not alter the lexical semantics, (48a) yields an adjective as in (49b):
a. $\llbracket$ Asp $\rrbracket=\lambda P[P]$
b. $\llbracket$ Asp $\sqrt{\text { FLAT } \rrbracket}=\lambda x \lambda s\left[\right.$ flat $\left.^{\prime}(x, s)\right]$

To produce the simple adjective meaning we assume (Cresswell 1977; von Stechow 1984; Kennedy 1997; inter alia) a head pos that sets the root-supplied standard equal to the positive standard (where $\mathbf{d}_{x}^{\delta_{P}}$ for scalar stative predicate $P$ is defined as its standard $\mathbf{d}_{x}^{\delta}$, for $\boldsymbol{\delta}_{P}$ the dimension of $P$ ):
a. $\llbracket p o s \rrbracket=\lambda P \lambda x \lambda s\left[P(x, s) \wedge p o s^{\prime}(P)\right]$
b. For any $P, \operatorname{pos}^{\prime}(P)$ is true iff $\mathbf{d}_{x}^{\boldsymbol{\delta}_{P}}=\operatorname{stnd}_{\text {pos }}^{\prime}\left(\boldsymbol{\delta}_{P}\right)$. 
Applying (50a) to (49b) and then applying the output to the patient the rug and existentially binding $s$ produces the following (ignoring tense and aspect and assuming the copula is also the identify function):

$$
\begin{aligned}
& \text { I[ The rug is }[\operatorname{pos}[\mathrm{Asp} \sqrt{\mathrm{FLAT}}]]] \rrbracket \\
& =\exists s\left[\operatorname{flat}^{\prime}\left(\mathbf{r u g}^{\prime}, s\right) \wedge \operatorname{pos}^{\prime}\left(\lambda x \lambda s\left[\operatorname{flat}^{\prime}(x, s)\right]\right)\right]
\end{aligned}
$$

"There exists a state $\mathrm{s}$ in which there is a degree $d \geq \mathbf{d}_{\mathrm{r}}^{\text {FLATNESS }}$ and $s$ is the state of the rug holding degree $d$ of FLATNESS and $\mathbf{d}_{\mathbf{r}}^{\text {FLATNESS }}=\operatorname{stnd}_{\text {pos }}^{\prime}$ (FLATNESS)." $\approx$ "There is a state $s$ in which the rug holds a degree $d$ of FLATNESs at or above the positive standard for FLATNESS."

The ultimate interpretation depends on how we interpret the positive standard as above (see e.g. (42)). Alternatively, a comparative or superlative head could apply above AspP instead, setting the standard explicitly as the degree of the thing(s) the patient is being compared to. We ignore the details of this here.

For change-of-state verbs, $v_{\text {become }}$ imposes a distinct set of conditions, as in (52). In particular, $v_{\text {become }}$ ensures there is an event of change at the end of which the patient holds the relevant scalar property to a degree above the root-supplied standard and at the beginning of which the patient holds the relevant scalar property to a degree below the root-supplied standard, where the root-supplied standard is in turn defined as the verbal standard. Additionally, the event must stand in an FPR to the theme and an appropriate subpart of the scale, where for any scalar state $s$ the individual $\mathbf{x}_{s}$ is its patient, $\boldsymbol{\delta}_{s}$ is its scalar dimension, $\mathbf{d}_{s}$ is the degree to which $\mathbf{x}_{s}$ holds $\delta_{s}$ in $s$, and $\mathrm{S}_{s}$ is the set of degrees for the scale of $s$. Assuming uniqueness of participants for any eventuality as per Krifka (1998: 209, (41)), these will correspond to the $x$ argument of $v_{\text {become }}$, the dimension $\delta$ and the set of degrees $S$ of the $P$ argument of $v_{\text {become }}$, and whatever degree $d$ the stative predicate $P$ entails $x$ holds on $\delta$, respectively: ${ }^{26}$

a. $\llbracket v_{\text {become }} \rrbracket=\lambda P \lambda x \lambda e \exists s[$ become $(s, e) \wedge P(x, s)]$

b. for all $e, s$, become $(s, e)$ is true iff $s$ holds at the end of $e$ and at the beginning of $e$ there is a state $s^{\prime}$ such that there is a degree $d^{\prime}$ on $\delta_{s}$

\footnotetext{
26 This makes our semantic analysis in some ways comparative in being built on a scalar semantics, much in the way Bobaljik (2012: Chapter 6) suggests based on crosslinguistic suppletive morphological facts between comparative forms and change-of-state verbs (at least absent other morphosemantic routes to a change-of-state verb, as Bobaljik 2012: 198-9 discusses for the inceptive, among other possibilities). Our syntactic representations, however, do not transparently embed the comparative. While suggesting that he believes the evidence points in that direction, Bobaljik (2012: 205-7) does accept that the situation is somewhat complicated and less straightforward than the evidence for his syntactic relationship between positive, comparative, and superlative form adjectives, and does allow that his morphological observations might be triggered by the right kind of scalar semantics for state change, much like the one we are assuming. The relationship between comparatives and their change-of-state verb counterparts, both morphosyntactically and semantically strikes us as a fascinating outstanding question, albeit one we must leave open here.
} 
where $\mathbf{d}_{\mathbf{x}_{s}}^{\delta_{s}}>d^{\prime}$ and $\mathbf{d}_{\mathbf{x}_{s}}^{\delta_{s}}=\operatorname{stnd}_{\mathrm{V}}^{\prime}\left(\boldsymbol{\delta}_{s}\right)$ and $e$ is FPR-related to $\mathbf{x}_{s}$ and the continuous, ordered set of degrees $S^{\prime} \subseteq \mathrm{S}_{s}$ of $\boldsymbol{\delta}_{s}$ containing $d^{\prime}$ and whose maximal degree is $d_{s}$.

Since $P$ entails that $\mathbf{d}_{s}$ is equal to or above the root-supplied standard and $d^{\prime}$ is below the root-supplied standard it follows that $\mathbf{d}_{s}>d^{\prime}$, i.e. a change occurred. The root-supplied standard is in turn equated with the verbal standard, giving rise to the various readings discussed above (e.g. as in (45)). This would produce the following when applied to $\sqrt{\text { FLAT }}$ and the rug, with all event variables bound off:

$\llbracket\left[\right.$ the rug $\left.\left[v_{\text {become }} \sqrt{\text { FLAT }}\right]\right] \rrbracket=\exists e \exists s\left[\operatorname{become}^{\prime}(s, e) \wedge\right.$ flat $^{\prime}\left(\right.$ rug $\left.\left.^{\prime}, s\right)\right]$

"There is an event $e$ and state $s$ such that $s$ holds at the end of $e$ and at the beginning of $e$ there is a state $s^{\prime}$ such that there is a degree $d^{\prime}$ of FLATNEss where $\mathbf{d}_{\mathrm{r}}^{\text {FLATNESS }}>d^{\prime}$ and $\mathbf{d}_{\mathrm{r}}^{\text {FLATNESS }}=\operatorname{stnd}_{\mathrm{V}}^{\prime}$ (FLATNESS) and $e$ is FPR-related to the rug and the continuous, ordered set of degrees $S^{\prime} \subseteq S_{s}$ of FLATNESS containing $d^{\prime}$ and whose maximal degree is the degree $d$ that $x$ holds of FLATNESs in $s$, and there is a degree $d \geq \mathbf{d}_{\mathrm{r}}^{\text {FLATNESS }}$ and $s$ is the state of the rug holding degree $d$ of FLATNESs."

$\approx$ "There is an event $e$ in which the rug goes incrementally from holding some degree $d^{\prime}$ of FLATNESs below the verbal standard for FLATNESs to some degree $d$ of FLATNESS above the verbal standard."

Since flatness has a maximum endpoint the default reading is that the rug is completely flat, but if context intervenes it could be something else. Most critically for the rest of this study, this analysis imports the homomorphic analysis of telicity and durativity discussed in $\$ 1.6 .1 .1$ into an event structural approach, by building scales into stative roots and an FPR into $v_{\text {become }}$.

It also captures the crucial again facts discussed in \$1.6.1.2. If again scopes over the root we derive the following:

$$
\llbracket[\sqrt{\text { FLAT again }}] \rrbracket=\lambda z \lambda e^{\prime \prime \prime}\left[\operatorname{flat}^{\prime}\left(z, e^{\prime \prime \prime}\right) \wedge \partial \exists e^{\prime \prime}\left[e^{\prime \prime} \ll e^{\prime \prime \prime} \wedge \text { flat }^{\prime}\left(z, e^{\prime \prime}\right)\right]\right]
$$

This ensures that $z$ is in a state of holding a degree of flatness above some standard and there was a previous state of $z$ being above that standard, though the two states do not have to be of the rug holding the exact same degree of flatness. If Asp and pos applied the standard would be the positive standard:

[I The rug is $\left[\right.$ pos $[$ Asp $\sqrt{\text { FLAT again }]]}] \rrbracket=\exists e^{\prime \prime \prime}\left[\right.$ flat $^{\prime}\left(\right.$ rug $\left.^{\prime}, e^{\prime \prime \prime}\right) \wedge \partial \exists e^{\prime \prime}\left[e^{\prime \prime} \ll\right.$ $e^{\prime \prime \prime} \wedge$ flat $\left.\left.^{\prime}\left(\operatorname{rug}^{\prime}, e^{\prime \prime}\right)\right] \wedge \operatorname{pos}^{\prime}\left(\lambda z \lambda e^{\prime \prime \prime}\left[\operatorname{flat}^{\prime}\left(z, e^{\prime \prime \prime}\right)\right]\right)\right]$

$\approx$ "There is a state $e^{\prime \prime \prime}$ in which the rug holds a degree $d$ of FLATNEss above the positive standard for FLATNESS and it is presupposed that it previously held a (possibly) different degree $d$ of FLATNEss above the positive standard for FLATNESS in some state $e^{\prime \prime}$." 
Crucially again scopes over the comparison to the positive standard by virtue of scoping over comparison to the root-supplied standard, which is equated with the positive standard. If $v_{\text {become }}$ instead applies above (54) we get (56):

【the rug $\left[v_{\text {become }}[\sqrt{\text { FLAT again }]}] \rrbracket=\exists e \exists s\left[\operatorname{become}^{\prime}(s, e) \wedge \operatorname{flat}^{\prime}\left(\operatorname{rug}^{\prime}, s\right) \wedge\right.\right.$ $\partial \exists e^{\prime \prime}\left[e^{\prime \prime} \ll s \wedge\right.$ flat $^{\prime}\left(\right.$ rug $\left.\left.\left.^{\prime}, e^{\prime \prime}\right)\right]\right]$

$\approx$ "There is an event $e$ in which the rug goes incrementally from holding some degree $d^{\prime}$ of FLATNESs below the verbal standard for FLATNESs to some degree $d$ of FLATNESs above the verbal standard for FLATNESS, and it is presupposed that it previously had held some (possibly) different degree $d$ of FLATNESS above the verbal standard for FLATNESS in some $e^{\prime \prime}$."

Again, the reading follows from how the standard is interpreted, as discussed above. In sum, we can define a notion of change of state that builds on a scalar analysis in ways that can incorporate its lexical aspectual correlates and also correctly captures the semantics of restitutive modification.

The only remaining question is what the definitions of in an hour and for an hour modifiers are, and where they attach. Since this is not our interest here we simply adopt for convenience the essence of the definition of for an hour modifiers in Krifka (1998: 215, (55)), where, roughly, for an hour presupposes that the predicate it applies to is atelic and the event measures an hour long (which will also ensure durativity). Krifka's (pp. 215-8) analysis of in an hour is that the temporal trace of the event occurred during a one-hour period and (through denial of scalar alternatives) not any larger or smaller interval. For our purposes we simply assume that in an hour presupposes that the predicate it applies to is telic and says that the event culminated at the end of a one-hour interval, but leaving open how long the event is, allowing durative or punctual readings. As for where these modifiers attach in the event structure we defer this discussion to $\$ 1$ 6.2.2. We now turn to the question of how to define causation.

\subsubsection{Causation and its role in verb meaning}

\subsubsection{Theories of causation}

How exactly to interpret cause' hinges on what exactly it means for one thing to have caused another. This is a matter of profound philosophical, physical, psychological, and linguistic complication. Although there has been a great deal of work on this issue, there is little in the way of agreement. Copley and Wolff (2014) provide a recent, cogent overview of the issues, dividing theories into two broad types: dependency theories and production theories (see also Dowe 2000: 14-88). The former have it that a causal relation holds between two objects (e.g. 
events or propositions, depending on one's theory) if one depends on another in some particular way. How exactly this dependency relation should be articulated is a point of contention. Since at least Hume (2007[1784]) some have argued for a logical dependency relation, so that A causes B iff A is necessary and/or sufficient for B. An alternative, articulated primarily in an influential paper by Lewis (1973) (see also Dowty 1979: 99-110, Lewis 2000), articulates instead that the dependency must be built on a relation of counterfactuality (building on Stalnaker 1968). In the simplest case, A causes B iff for A to not have occurred entails that B would not have occurred, though as Lewis makes clear a more articulated model should incorporate causal chains $\mathrm{C}_{1} \ldots \mathrm{C}_{n}$ where $\mathrm{C}_{1}$ causes $\mathrm{C}_{n}$ iff for all $i(1 \leq i \leq n)$ for $\mathrm{C}_{i}$ not to have occurred entails that $\mathrm{C}_{i+1}$ would not have occurred (see e.g. Lewis 1973: 563; Dowty 1979: 99-110; Kratzer 2005: 197-8; inter alia). Still others (see e.g. Reichenbach 1956; Suppes 1970; Eells 1991) couple dependency relations with probabilities, so that causation of B by A is tied to an increase in the likelihood of B's holding if $\mathrm{A}$ is more likely to hold.

While there is much intuitive appeal to dependency as a basis for a theory of causation, as Copley and Wolff discuss in detail dependency theories struggle with various kinds with preemption-cases where A would cause B had C not intervened to cause B instead (late preemption) or where A causes B but had A not occurred $C$ would have caused B (early preemption). In such cases, depending on the variety of preemption and precisely how the dependency is defined the dependency is broken but the effect remains, causing problems for the correct articulation of the dependency, and more generally in capturing what are perceived to be intuitions about causation.

A class of competitors to dependency theories are production theories in which there is transmission of some mark in a causal process (e.g. Salmon 1984: 139-57), or a transmission of energy (Fair 1979) or other conserved quantity between entities or events (e.g. as per Dowe 2000: 89-122; Kistler 2014: 92), or some imparted force (e.g. as per Talmy 1988: 67-8, 2000: Vol. II, 428-30, 471-546; Croft 1991: 159-82, 2012: 197-205; Wolff 2007; Wolff et al. 2010; Wolff 2014; Copley and Harley 2014, 2015). Theories of this sort have it that some positively defined entity of some sort is expended or transfered that in turn yields the effect as a result. Such analyses can explain a variety of temporal properties (in particular that the cause precedes the effect; Lagnado et al. 2007: 159-61, though see Dowe 2000: 176-210) and physical properties of causation that do not immediately have an explanation on dependency theories (in particular that cause and effect are linked "through a chain of physical connections"; Copley and Wolff 2014: 24). Additionally, they do not suffer from the problems with preemption that raise questions for dependency theories. Production theories have other problems, however, including how to deal with instances of apparent causation in which the causer is abstract or when there is lack of physical proximity (which Copley and Wolff: 24 dub "spooky action at 
a distance," borrowing Albert Einstein's pithy term for quantum entanglement), and also the general problem that things like force and energy are not objectively observable.

Copley and Wolff survey a wide variety of approaches to both kinds of theories, alongside their strengths and weaknesses. What is clear from their discussion is that the nature of causation, and therefore how precisely to interpret the truth conditions of cause', is very much a large, outstanding question in physics, lexical semantics, the philosophy of language, and psychology more broadly. This is not a problem that we can hope to solve in the context of this book. For our purposes, therefore, we do not make a definitive decision here about how exactly causal dependencies are to be analyzed.

Rather, we modestly assume that cause' relates two eventualities together in either some kind of dependency or production model where we assume that for a (di)transitive caused change-of-state verb the subject is a participant of the causing event and thus depending on the properties of this event there may be restrictions on types of possible subjects, while the objects are participants of the caused event and thus depending on the properties of this event there may be restrictions on types of possible objects (though in some cases it may be that an object is also a participant of the causing event and thus subject to conditions from whatever type of event it is, something we only discuss when relevant). We furthermore assume that for (di)transitive change-of-state verbs if there are two (or more) event structural subevents in the overall event described by the verb, causation is the relation that relates the subevents together, regardless of how exactly that relation is modeled, unless there is a specific reason to assume some other relation, whatever it may be.

Setting the specific model aside, there are further distinctions in types of causation that must be accounted for, two of which we mention here as they are germane to the discussion. One especially well discussed distinction between types of causation is illustrated by the distinction between lexical causatives like caused change-of-state verbs and verbs that form periphrastic causative constructions as in the following:

(57) a. Pat killed the soldier (with a gun).

b. Pat caused the soldier to die.

The verb kill generally seems to require a fairly tight connection between whatever it is the agent does and the death of its patient, whereas for cause to die the connection can be looser. For example, the causing and caused events can be fairly temporally disconnected with cause to die but cannot be for kill (Fodor 1970: 432-3):

(58) a. \#John killed the soldier today by shooting him with a gun yesterday.

b. John caused the soldier to die today by shooting him with a gun yesterday. 
A common way of analyzing this is in terms of directness of causation (e.g. as per Fodor 1970: 432-3; Shibatani 1976: 14-6; Bittner 1998: 12), where lexical causatives require the causal relationship between the causing and caused events to be direct while periphrastic causatives allow more indirect causation (though there may be intermediate levels of directness; see e.g. Shibatani and Pardeshi 2001: 6-10 on "sociative" causation in which the causer and the patient/causee work together towards achieving a result). Since our primary concern in this study is lexical causatives, we assume that whatever the truth conditions of cause $e^{\prime}$ are they will ensure relatively direct causation. (See Wolff 2003, however, for evidence that direct causation is not always categorically required for lexical causatives, as Bittner 1998: 2 also suggests, though for present purposes we assume it is in general the case.)

A second point of variation in studies on causation is whether the caused event must actually occur. In English it is typical for caused change-of-state verbs that it does, e.g. denying that this is the case yields a contradiction:

\#Pat (just) shattered the vase, but it is not shattered.

However, in some languages translation equivalents of English change-of-state predicates do not entail that the event obtains, a phenomenon known as "nonculmination" (e.g. Japanese in Ikegami 1985; Hindi in Singh 1998, Arunachalam and Kothari 2011; Thai in Koenig and Muansuwan 2000; Salish languages in Bar-el et al. 2005; Chinese in Koenig and Chief 2008; Korean in Beavers and Lee in press; see Demirdache and Martin 2015 and Koenig et al. 2016 for crosslinguistic summaries). This phenomenon can be illustrated in the following from KarachayBalkar (Turkic; spoken in the Caucasus), in which a predicate that translates into English as open the door does not generate an entailment that the door actually came to be open:

$\begin{array}{llll}\text { kerim } & \text { ešik-ni } & \text { ac-xan-di, } & \text { alaj boša-ma-Ban-di. } \\ \text { Kerim door-ACC } & \text { open-PFV-3sG but finish-NEG-PFV-3sG }\end{array}$

(lit.) 'Kerim opened the door, but did not finish.' (Tatevosov 2008: 396, (9))

However, this reading is not freely available. The most general claim regarding when non-culmination arises is the Agent Control Hypothesis of Demirdache and Martin (2015: 201, (20b)) wherein the subject must bear some kind of agentivity properties on non-culmination readings that do not necessarily obtain on culmination readings. As an illustration, consider Korean, where non-culmination readings require that the subject intend for the result to obtain (assuming intentionality is an agentivity property), whereas culmination readings do not require this. This is evidenced by the fact that in contexts where non-culmination is forced by denial of a result modifiers that mean "unintentionally" are unacceptable but modifiers meaning "intentionally" are acceptable (though they carry a flavor redundancy). 
If the result is known to have occurred, both types of modifiers are possible (with no redundancy):

a. ku-ka (?uytocekulo/?ilpwule/\#uytohacianhkey/\#silswulo)

he-NOM intentionally/on_purpose/unintentionally/by_mistake

changmwun-ul yel-ess-ta. kulena changmwun-i

window-ACC open-PST-DECL but window-NOM

yel-li-ci anh-ass-ta.

open-PASS-COMP NEG-PST-DECL

(lit.) 'He opened the window (intentionally/on purpose/unintentionally/ by mistake). But the window was not opened.'

b. ku-ka uytohacianhkey/silswulo/uytocekulo/ilpwule

he-NOM unintentionally/by_mistake/intentionally/on_purpose

changmwun-ul yel-ess-ta. kulayse changmwun-i

window-ACC open-PST-DECL so window-NOM

cokum/wancenhi yel-li-ess-ta.

a_little/completely open-PASS-PST-DECL

'He opened the window unintentionally/by mistake/intentionally/on purpose. So the window was a little/completely opened.'

(Beavers and Lee in press: 5-6, (8b), (9b), (10b))

Of course, intentionality is not a crosslinguistic requirement for non-culmination, nor even is agentivity. For example, Martin and Schäfer (2012: 252-6) argue that French and German offer verbs admit non-culmination readings but intentionality is not at issue, as with certain inanimate subjects:

Ces circonstances lui ont offert un super job; et
those circumstances her have offered a great job and
pourtant, contre toute attente, elle ne l' a pas
yet against all expectations she NEG it has NEG
pris.
taken

'These circumstances offered her a great job; and nevertheless, against all expectations, she didn't take it.'

(French)

(based on Martin and Schäfer 2012: 254, (15), glosses ours)

Rather, the non-culmination reading here seems to correlate instead with unexpectedness regarding the lack of a result from the speaker's perspective. Thus the conditions that give rise to non-culmination vary across languages.

Returning to theories of causation, a common assumption in much work on this topic is that the occurrence of a caused event is outscoped by some (sublexical) modality whereby the caused event obtains in some possible world and not 
necessarily the real world à la Koenig and Muansuwan (2000), Bar-el et al. (2005), Tatevosov (2008), Martin and Schäfer (2012, 2017), and Beavers and Lee (in press), inter alia (see also Dowty 1979 and Beavers 2011a, though see Copley and Harley 2014, 2015 for an alternative that relies on non-efficacious forces to model non-culmination in a force-theoretic analysis of causation). On a modal analysis crosslinguistic variation in the circumstances that give rise to non-culmination readings (e.g. intentionality or unexpectedness) follow from different modal forces and modal bases different languages may have for this modality. The upshot for our purposes is that the head that introduces simple causation and not much else-which we gave above as $v_{\text {cause }}$ - may not be the only causal head, and other causal heads may introduce an additional sublexical modality (as per Tatevosov 2008: 410), and there may even be different flavors of this (see Folli and Harley 2004 on flavors of $v$ more broadly). We will indeed ultimately posit a separate, modalized variant of $v_{\text {cause }}$ for English ditransitive verbs which show a type of nonculmination effect, though at least for the data discussed in this study just these two types of causal $v$ should be sufficient. ${ }^{27}$

In sum, we assume a model of causation that relates two eventualities together, each potentially distinct types of events that will impose distinction conditions on their participants, and among change-of-state events with multiple event structural subevents direct causation is the relation that relates the various subevents together. On a case by case basis the head that introduces the cause' relation may also introduce some type of sublexical modality deriving some type of nonculmination effect.

\subsubsection{Causation and aspect}

Before we conclude, we discuss one final issue, here regarding the connection of causation to lexical aspect. In particular, we had suggested in $\$ 1.6 .1 .1$ that durativity of change-of-state predicates hinge on (a) the mereological complexity of the patient and (b) the gradability of the scale of change, as analyzed by the Figure/Path Relation of Beavers (2012). However, Beavers only considered change-of-state predicates where the change was the entire event described by the verb, ignoring any contribution of causing events. A fact we will discuss extensively in Chapter 4 is that a change-of-state predicate can describe not just a change or just a change plus some unspecified cause but also a specific causing action, as in a resultative (e.g. Kim hammered the metal flat) or verbs that we claim violate Manner/Result Complementarity (e.g. braise as in Kim braised the lamb).

\footnotetext{
${ }^{27}$ Indeed, a still more comprehensive typology should presumably include more types of causation beyond degrees of directness and (non-)culmination, as per the work of Talmy (1976, 2000: Vol. II, 471-550) (see also Wolff 2003, 2007; Wolff and Song 2003; Wolff et al. 2010) that defines various subtypes of causation, prevention, and enabling within a single force-dynamic framework. These might all ultimately be analyzable as combinations of some basic, fundamental notion of causation plus possible additional operators to derive a full typology.
} 
In this case the mereological complexity of the additional causing actions could contribute to the mereological complexity of the overall event described by the verbal predicate and produce durative predicates even with an atomic theme and non-gradable scale.

That manner alone can determine durativity is shown by considering nonchange-of-state predicates that simply describe actions, including semelfactives like blink once which describe pure action events that are punctual vs. action verbs like dance or jog which describe complex actions. The former pattern with punctual change-of-state predicates in occurring with in an hour modifiers on an after reading and the latter take for an hour (like atelic predicates in general) and have a during reading: ${ }^{28}$

(63) a. Mary can blink once/clap once in an hour.

(after an hour)

b. Mary can dance/jog for an hour.

(for an hour)

Thus action complexity can contribute to durativity. If a predicate entails both some manner-such as a causing manner-and also a scalar change then both could contribute to durativity. We discuss cases of exactly this in Chapter 4, but the primary concern here is how to model this.

In principle, the simplest analysis is that all of the change-of-state and manner events entailed to be part of the causal chain by a single change-of-state predicate are somehow mereologically joined at the top $v \mathrm{P}$, which is thus a predicate over the joined events (e.g. that there is some whole event $h=e \oplus v$ in (24) that the top $v \mathrm{P}$ predicates of). On their telicity/durativity probing readings in an hour and for an hour modifiers apply here, thus taking into account the mereological complexity of all of the relevant subevents (with some condition that the final point of $h$ corresponds to the final point of $e$, so that telicity follows as above, and that no part of $v$ temporally follows the final point of $e$, to capture the condition in \$1.6.2.1 that causation either precedes or is contemporaneous with the caused event as per Lagnado et al. 2007: 159-161; see Jerro 2017a: 770 for a condition to this effect in an event semantic framework). ${ }^{29}$ Since we are not providing a detailed analysis of these two diagnostics we omit this formal detail but assume that in a complete implementation it would be a component.

\footnotetext{
${ }^{28}$ Punctual predicates are always telic since, being instantaneous, they entail an endpoint, something true regardless of whether they are punctual manner verbs like semelfactives or punctual changeof-state verbs like achievements, explaining why both take in an hour modifiers.

${ }^{29}$ Four an hour modifiers would scope over the root, however, for the state readings discussed in $\$ 1.3 .2$.
} 
With these preliminaries established, we are now in a position to turn to our primary case studies regarding hypotheses about root meaning. We begin with a discussion of Bifurcation in the roots of canonical change-of-state verbs, before turning to Bifurcation in ditransitives of caused possession and then questions of Manner/Result Complementarity. 



\section{2 \\ Entailments of change in the roots of change-of-state verbs}

\subsection{Two types of change-of-state verbs}

As evidenced by Chapter 1, no class of verbs has had its lexical semantics better studied than change-of-state verbs, i.e. verbs which lexically entail some kind of (scalar) change in one of their participants. Such verbs have been at the forefront of work in nearly every theoretical approach to event structure and are widely assumed by theoreticians working in a variety of frameworks to motivate a decompositional approach to verb meaning in some form or another. As discussed in $\$ 1.3$, the starting point of the literature on these verbs has been the separation of the change component of the verb's meaning from the stative component, with change-of-state verbs differing from one another purely in the kind of state the verb entails change into, where in the simplest case the root just denotes a state and the change comes from the template.

Taking this intuition to its extreme, we might expect that all cases are exactly like this, which would represent an example of the Bifurcation Thesis of Roots of (Embick 2009: 1) discussed in $\$ 1.4 .1$, repeated here:

(1) The Bifurcation Thesis of Roots: If a component of meaning is introduced by a semantic rule that applies to elements in combination [i.e. is introduced by a functional head $-\mathrm{B} / \mathrm{KG}$ ], then that component of meaning cannot be part of the meaning of a root.

In this chapter, we begin our argument against this view, making the explicit case for templatic meaning in the root by arguing that at least some change-ofstate verbs have entailments of change arising from the meaning of the root itself rather than (just) from the template. In so doing, we make an argument against Bifurcation from possibly the most obvious empirical domain where it would be expected to hold if it were correct. ${ }^{1}$

\footnotetext{
1 This chapter represents a significantly revised and expanded version of Koontz-Garboden and Beavers (2017), which is presaged by Koontz-Garboden and Levin (2005) and Koontz-Garboden (2005, 2006, 2007); see also Beavers et al. (2018).
} 
Our core observation is that there is a split between two types of change-ofstate verbs in terms of whether the entailment of change is introduced solely by the template or an entailment of change is (also) present in the root itself. In particular, building on Dixon (1982: 50), we propose a distinction between change-of-state verbs whose roots describe Dixon's Property Concept (PC) states, which in English correspond to Levin's deadjectival change-of-state verbs such as those in (2), vs. change-of-state verbs based on Dixon's "states that are the result of some action" (Dixon 1982: 50), which in English correspond to Levin's (1993) non-deadjectival change-of-state verbs such as those in her break, cook, and kill verb classes, among others, as in (3): ${ }^{2}$

(2) Deadjectival COS verbs (Levin 1993: 245)

awaken, brighten, broaden, cheapen, coarsen, dampen, darken, deepen, fatten, flatten, freshen, gladden, harden, hasten, heighten, lengthen, lessen, lighten, loosen, moisten, neaten, quicken, ripen, roughen, sharpen, shorten, sicken, slacken, smarten, soften, stiffen, straighten, strengthen, sweeten, tauten, thicken, tighten, toughen, weaken, widen,...

(3) Non-deadjectival COS verbs

a. Levin's (1993: 241) break verbs: break, chip, crack, crash, crush, fracture, rip, shatter, smash, snap, splinter, split, tear

b. Levin's cooking verbs (Levin 1993: 243): bake, barbecue, blanch, boil, braise, broil, charbroil, charcoal-broil, coddle, cook, crisp, deepfry, fry, grill, hardboil, poach,...

c. Verbs of killing (Levin 1993: 230-3): crucify, electrocute, drown, hang, guillotine,...

d. inter alia

Crucially, while virtually all of the roots of the verbs in (2) can readily be found in contexts lacking any inference of change, the roots of the verbs in (3) cannot (modulo some exceptions, as we discuss further below). The fact that roots in the latter class cannot be disembodied from an entailment of change suggests that such an entailment must be part of the meaning of the root itself. Presaging this conclusion somewhat, we therefore refer to the roots of the verbs in (2) as PC roots and those in (3) as result roots.

Having made the semantic case for two distinct classes of roots in $\$ 2.2$, we consider in $\$ 2.3$ whether there are any morphophonological correlates of it. $^{3}$

\footnotetext{
2 The term "property concept" was not actually introduced by Dixon (1982) himself to demarcate the class of states "naturally described by adjectives" (Dixon 1982: 50), as he says, but rather by Thompson (1988: 168), whose term for Dixon's class of states has since gained currency.

${ }^{3}$ Calling the class in (2) "deadjectival” is simply following Levin's (1993) terminology, and should not be taken as our prejudging the morphological nature of the members of the class.
} 
We present evidence from the morphological typology of the two verb classes which shows that verbs based on roots in the different classes have distinct morphological composition across various templates they occur in, a fact which can already be seen in (2) and (3) and which repeats itself in different ways in other languages. We then turn to the analytical consequences of these observations. We first articulate in $\$ 2.4$ a variety of ways of theoretically accommodating what we take to be the most intuitive consequence of the facts that we observe, namely that while PC roots lack templatic entailments, result roots overwhelmingly come endowed with an entailment of change, a fact which taken at face value falsifies Bifurcation. We consider how this idea can be implemented in strictly nonlexicalist and lexicalist event structural frameworks, plus frameworks that mix lexicalist and non-lexicalist assumptions, discussing the strengths and weaknesses of each type of theory. In each case the upshot is that Bifurcation must be rejected, independent of what kind of framework the question is approached from, the choice of which we believe is an issue that ultimately hinges on a much broader set of theoretical considerations and assumptions that are independent of the issues we explore here. The sum of our observations ultimately yields a verb class typology with both semantic and morphological predictions derived from a typology of roots that is orthogonal to templatically defined typologies, where the combination of what the root brings to bear and what the template brings to bear ultimately define the verb class typology, something we consider the consequences of more in $\$ 5.4$.

Notwithstanding the observations we believe motivate the rejection of Bifurcation, in $\$ 2.5$ we also explore what it would take to preserve it in the face of these facts, articulating theories that would fundamentally alter the meanings of result roots, that would give an allosemic treatment to the relevant templatic heads, and theories that would allow roots to select for the templates they can/cannot appear in, coupled with other assumptions designed to get the data right under the assumption of Bifurcation. As we discuss, these approaches all come with theoretical and empirical shortcomings. As a consequence, our position is that it difficult to avoid the conclusion that result roots lexically entail change of state and that more broadly root and template meaning cannot be universally bifurcated. Nonetheless, we argue in $\$ 2.6$ that despite the fact that result roots have change in their meanings, templates are still needed in the full analysis of the verbs they derive. A deeper question is why roots would violate Bifurcation and entail both a state and a change into that state simultaneously. We ultimately suggest this largely has to do with the existence of kinds of states that are conceived of as only arising from some change (or possibly some specific action, as we discuss in Chapter 4). However, we defer this deeper discussion to $\$ 5.2$, after we have fully outlined our case for what roots can and cannot entail. Here we just focus on justifying that Bifurcation does not hold in the roots of change-of-state verbs. 


\subsection{Lexical semantic consequences of Bifurcation}

In order to probe the meanings of change-of-state verb roots, we first focus on the adjectival structures built from the same roots. The reason is that the templates that give rise to adjectival uses of the same roots are in many cases uncontroversially void of the types of functional structure that would introduce templatic meanings such as change of state. As a consequence, looking at these uses allows us to be certain that whatever lexical entailments such adjectives have must come from the root and not some templatic head.

For clarity, we consider Embick's (2004) analysis of the roots of change-of-state verbs and the adjectives that can be built on these roots. Although other analyses differ in (irrelevant) details, this analysis is representative of the assumptions that are needed in order to make the Bifurcation thesis work. ${ }^{4}$ Our point of departure is two adjectival structures. The first is the structure used for adjectives that describe "basic states." These have a stative (precategorial) root Merging directly with an adjectivalizing morpheme called Asp in Embick's theory. Asp crucially adds no non-vacuous templatic meaning to the event structure, its sole purpose being to make an adjective out of a precategorial state-denoting root so that it can predicate of a DP argument: ${ }^{5}$

(4) Basic states (aka adjectives, statives, etc.) (Embick 2004: 363)

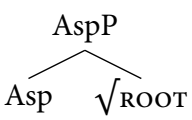

Basic states contrast with result states, corresponding to adjectives that are (descriptively speaking) derived from verbs as in (5) and which include the functional head introducing change-of-state entailments. Embick (2004: 367) calls the this head FIENT; we instead call it $v_{\text {become }}$ as per $\$ 1.3:^{6}$

\footnotetext{
${ }^{4}$ One of the key ways in which analyses such as Hale and Keyser (2002), Baker (2003), and others differ from one another is in the syntactic nature of the root itself, and whether it is categorial (e.g. Hale and Keyser 2002) or not (Embick 2004). Theories also differ in their assumptions about the nature of the functional structure (e.g. whether entailments of causation and agentivity are bifurcated as in Pylkkänen 2008 or not) and in whether any word formation is non-syntactic, among other things. None of these debates affect the key assumption, common among many theories, that change-of-state verb roots are semantically stative and thus in principle should be able to appear in contexts where no entailment of change arises (absent some combinatoric stipulations like those discussed in \$2.5).

${ }^{5}$ Here and throughout we have made some minor modifications to trees taken from Embick (2004) and other sources to make them consistent with the notational conventions we have introduced, primarily in indicating bar-levels on mother nodes of head-complement structures to more clearly distinguish it from adjunction and in the notation used for indicating roots. These modifications are purely notational and do not affect any of the claims being made.

${ }^{6}$ Embick (2004: 363) also assumes that the Asp heads in (4) and (5) respectively may be different from one another, though it is not entirely clear why given his claim that the same morphology can realize both with result roots (e.g. crack-ed, broke-en, etc.). Any such differences that may exist, whatever
} 
(5) Result states (Embick 2004: 367)

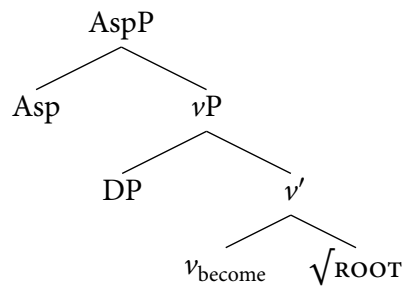

Finally, all roots of change-of-state verbs are purely state denoting on any analysis assuming Bifurcation, and only acquire an entailment of change through Merge

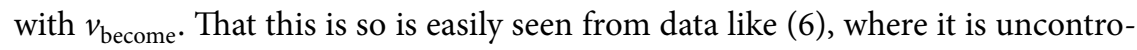
versial that the change entailment comes from become:

(6) Kim's face became red.

Bifurcation has it that any meaning that can be introduced syntactically must be introduced syntactically. It thus follows that the entailments of change in (7a) and (7b) are also introduced syntactically, and not by the root:

(7) a. Kim's face reddened, after the embarrassing entrance.

b. The vase broke.

For (7a) it is sometimes assumed, as in Embick, that the -en morphology realizes $v_{\text {become }}$. In (7b), by contrast, the assumption is that it is phonologically null. The key claim of Bifurcation, again, is that in all of (6)-(7) the change-of-state entailment is introduced in the syntax by $v_{\text {become }}$ and not by the root, whether there is any overt morphophonological evidence for $v_{\text {become }}$ or not.

On the assumption that Asp in (4) and $v_{\text {become }}$ in (5) subcategorize (in whatever sense is appropriate in a given framework) for a stative root, and given that the roots of all change-of-state verbs are by necessity stative, nothing prevents the roots of all change-of-state verbs from appearing in both adjectival structures. This means that the roots of all change-of-state verbs should be found in adjectival structures both with entailments of change as in (5) and without them as in (4). With PC roots we can transparently see from their morphological structure that they do appear in both syntactic contexts. This is evidenced by the contrast between flat, which realizes (4), with a null exponent for the Asp head, and flattened, which realizes (5), with an overt -ed exponent for the Asp head (Embick 2004: 364-8). With result roots, conversely, we see no contrast. The only adjectival 
forms consistently bear the morpheme -ed (and allomorphs), as with cracked. However, this does not mean they only realize the structure in (5). An alternative possibility, proposed by Embick (2004: 358), is that a form like cracked realizes both (4) and (5), with -ed being the realization of Asp in both structures, and the $v_{\text {become }}$ in (5) simply having no phonological realization. This is in contrast with the situation with PC roots, where $v_{\text {become }}$ is (often) realized as $-e n$.

Summarizing, on Embick's analysis all change-of-state roots appear in both adjectival structures, albeit differing in their morphology when they do:

\begin{tabular}{l|ll} 
& $\sqrt{\text { FLAT }}$ & $\sqrt{\text { CRACK }}$ \\
\hline Basic adjective formed by (4) & flat & cracked \\
Result adjective formed by (5) & flattened & cracked
\end{tabular}

With this as background, we now consider three predictions about entailment behavior that a bifurcated analysis gives rise to in the domain of change-ofstate verbs, ultimately arguing that the predictions are incorrect. We note at the outset that there are some complexities with the judgments on the relevant data, partly owing to issues with various tests for the relevant semantic entailments, but also partly owing to potential interference from alternative interpretations of the data in terms of what counts as a change of state and partly to some degree of speaker-to-speaker and root-by-root variation. We thus begin by giving our own judgments and those of others we have consulted for relevant illustrative roots on the appropriate readings in $\$ 2.2 .1$, discussing issues with the various tests as we proceed. We return to alternative interpretations and speaker/root variation in $\$ 2.2 .2$ and $\$ 2.2 .3$ respectively.

\subsubsection{Three semantic predictions}

The first semantic prediction is that morphologically basic adjectives (e.g. flat) will not entail a prior change. This is because the structure in (4) contains no $v_{\text {become }}$ and assuming Bifurcation the root itself cannot introduce an entailment of change. This prediction is clearly borne out. For example, on the simplest analysis the corresponding inchoative for each such adjective entails that simply a change into the relevant state occurred and nothing else (the standard position going back to Lakoff 1965 and the position assumed in $\$ 1.3$ ). If the adjective itself has no change in its meaning, then asserting the adjective while denying the inchoative should be acceptable, where the scope of negation is over the coming about of the state and not the state itself. Using red, bright, long, darken, and loose as case studies, the data in (9) show that the states described by such adjectives can hold of an individual at the same time that it can be felicitously denied with the derivationally related inchoative verb that the state described by this adjective arose through some change: 
(9) a. The red dirt has not reddened.

b. The bright photo has not brightened.

c. The long river has not lengthened.

d. The dark sky has not darkened.

e. The loose rope has not loosened.

This provides some evidence that these adjectives do not entail change.

That said, some analyses of inchoatives in some languages posit that in addition to entailments of change they also have entailments of causation as well, as observed for Spanish anticausatives by Koontz-Garboden (2009a: 112-9). If that analysis applies to English inchoatives as well, it could be that discourses like those in (9) are acceptable for the wrong reason: perhaps the negation is interpreted as denial that there is a causer of the change rather than denial of the change itself. Indeed, the nature of the lexical semantics of inchoatives is very much a matter of debate (for extensive discussion see Koontz-Garboden 2009a, Horvath and Siloni 2011, 2013, Beavers and Koontz-Garboden 2013a,b, Lundquist et al. 2016) and now seem likely to be subject to crosslinguistic variation (Lundquist et al. 2016). While the simple analysis assumed in $\$ 1.3$ might be reasonable for English, it likely is not for all languages, a fact which means this diagnostic might not fully generalize. Still further, there are many change-of-state verbs for which an inchoative is unavailable altogether for reasons having to do with the lexical semantics of the verb or other idiosyncratic factors. For example, causatives whose external arguments are thematically highly specified, like agent-subject verbs such as murder, generally do not have inchoative forms (see Haspelmath 1993: 94, (16) and Levin and Rappaport Hovav 1995: 105, inter alia; see Schäfer 2009: 653 for an overview discussion and references). Similarly, sometimes an inchoative form is missing for purely idiosyncratic reasons (e.g. destroy does not have one despite otherwise fitting the profile of a verb that should). In such cases a test frame like (9) is impossible to even construct.

For both of these reasons, we should seek out additional diagnostics for the testing of an entailment of change. One possibility is to use denial of an associated passive on the reading of denial of a change, since virtually all verbs have passive forms, even those lacking inchoative forms. However, this diagnostic too must be used with care, since passives generally always entail the presence of an additional causer participant, and thus denial of the passive form always has another reading in which the denial is not of a change of state of but of some external causer of the change of state. Thus the intended reading is that no event at all of the relevant sort occurred, something that might be brought out better with a modifier such as at all or in any way:

(10) a. The red dirt has never been reddened (at all/in any way).

b. The bright photo has never been brightened (at all/in any way).

c. The long river has never been lengthened (at all/in any way). 
d. The dark sky has never been darkened (at all/in any way).

e. The loose rope has never been loosened (at all/in any way).

(on intended reading)

Here again the adjectival state may hold but an event of change denied.

A third diagnostic which we believe does not suffer from the problems of the previous two is embedding of an NP with the adjectival modifier to be tested in the frame has never undergone a Ving where $\mathrm{V}$ is a verb derivationally related to the adjective whose inferential behavior is being tested. As the data in (11) show, this frame also leads to no contradiction with ordinary basic adjectives, again consistent with Bifurcation:

(11) a. The red dirt has never undergone a reddening.

b. The bright photo has never undergone a brightening.

c. The long river has never undergone a lengthening.

d. The dark sky has never undergone a darkening.

e. The loose rope has never undergone a loosening.

Taken together, controlling for unwanted readings and gaps in paradigms of which verbs show which forms, these examples all do suggest that basic adjectival forms of PC roots do not entail a change. Of course, still further tests could be devised that show the same point, e.g. it is not contradictory to say The red dirt never became red(der), among other things. For now we stick with the three diagnostics we have outlined here.

The second correct prediction of Bifurcation is that denial of an entailment of change with the same roots in the deverbal structure in (5) does lead to contradiction, suggesting that the deverbal structure includes $v_{\text {become }}$ and thereby the entailment of change $v_{\text {become }}$ introduces. ${ }^{7}$ The data in (12)-(14), using the three main diagnostics laid out above, bear this prediction out: ${ }^{8}$

\footnotetext{
7 The deverbal adjectives under discussion are participial forms, which as Kratzer (2000: 385-90) notes have two possible readings, a resultant state and a target state (building on Parsons 1990: 234-5). The distinction is described as one having to do with whether the state is reversible or not. So far as we can tell, the question of whether an adjective lexically entails a change or not is independent of the target/resultant state distinction, though the states we are interested in are largely if not entirely target states. We therefore do not discuss this distinction here.

${ }^{8}$ We use attributive modification here, though Embick (2004: 357, fn. 1) suggests that this is complicated by the fact that participles generally show both adjectival (qua stative) and passive (qua eventive) senses as seen in predicative positions, though in predicative position these two readings can be disambiguated and thus controlled for. For attributive modification the relevant disambiguation strategies are not available, yet we are interested only in the adjectival reading. However, if purely adjectival uses exist in general, and attributive position is an independently known adjectival position, then regardless of the availability of passive participle senses adjectival participle senses should be possible here as well, yet no acceptable readings of the relevant diagnostics are possible. Still, we could instead reconfigure the diagnostics to use predicative constructions as part of relative clause modifiers, with present tense copulas to push the reading toward an adjectival one. In such cases the judgments
} 
(12) a. \#The reddened dirt has not reddened.

b. \#The brightened photo has not brightened.

c. \#The lengthened river has not lengthened.

d. \#The darkened sky has not darkened.

e. \#The loosened rope has not loosened.

(13) a. \#The reddened dirt has never been reddened (at all/in any way).

b. \#The brightened photo has never been brightened (at all/in any way).

c. \#The lengthened river has never been lengthened (at all/in any way).

d. \#The darkened sky has never been darkened (at all/in any way).

e. \#The loosened rope has never been loosened (at all/in any way).

(on intended reading)

(14) a. \#The reddened dirt has never undergone a reddening.

b. \#The brightened photo has never undergone a brightening.

c. \#The lengthened river has never undergone a lengthening.

d. \#The darkened sky has never undergone a darkening.

e. \#The loosened rope has never undergone a loosening.

In contrast to the clearly acceptable readings of basic PC root adjectives in these contexts, deverbal PC root adjectives sound awkward in all of them.

The final prediction of Bifurcation is that since adjectives based on result roots are structurally ambiguous between the templates in (4) and (5) they will not entail prior change since in any particular context the adjective could be realizing (4), which lacks $v_{\text {become }}$ and therefore a change entailment. Crucially, this prediction is not borne out-such adjectives generally do entail a change of the kind described by the verb they are derivationally related to (Koontz-Garboden 2005, 2010, Deo et al. 2011). Here we focus on shatter, crack, murder, barbecue, and cook. For these the inchoative test only applies to some of them-murder does not have an inchoative, and for many speakers barbecue does not either. For the other verbs, denial of the inchoative while asserting the seemingly deverbal adjective yields a contradiction:

(15) a. \#The shattered vase has never shattered.

b. \#The cracked bowl has never cracked.

c. \#The cooked chicken has never cooked.

Less complicated are the passive and undergone a Ving diagnostics, both of which clearly show that adjectives based on result roots do entail change: 
(16) a. \#The shattered vase has never been shattered (at all/in any way).

b. \#The cracked bowl has never been cracked (at all/in any way).

c. \#The murdered man has never been murdered (at all/in any way).

d. \#The barbecued chicken has never been barbecued (at all/in any way).

e. \#The cooked chicken has never been cooked (at all/in any way).

(on the intended reading)

(17) a. \#The shattered vase has never undergone a shattering.

b. \#The cracked bowl has never undergone a cracking.

c. \#The murdered man has never undergone a murdering.

d. \#The barbecued chicken has never undergone a barbecuing.

e. \#The cooked chicken has never undergone a cooking.

Thus it seems that adjectives formed from result roots entail change.

A potential objection is that this is a matter of context. Setting aside the continuing context, what does it mean to describe something with the attributive adjectives shattered or murdered or barbecued? The contexts here do not establish this, and perhaps absent appropriate context these sentences are all deemed unacceptable because the same surface term is juxtaposed twice, once asserted and once negated (in contrast with PC roots above, where there is a separate form for the basic stative that clearly indicates the relevant state without a change). But if we can isolate what the relevant states are that lack change and embed sentences such as these in contexts that clarify that these states hold, then under Bifurcation they should be acceptable. Our claim, of course, is that whatever the states are these roots entail that a change gave rise to them, and thus there will always be a contradiction with any context in which a change does not occur. However, if Bifurcation is correct then there must be some state not arising from a change that corresponds to these adjectives. Perhaps the clearest methodology for identifying such a state would be that the relevant state must be one that has the appearance of having arisen by an event of the sort described by the verbal form. For example, if one shatters a ceramic vase, then the vase will end up in numerous pieces, and as such if one sees ceramic pieces whose composition is such that they would appear to form a vase if put together in the right way, one might assume it is a shattered vase regardless of whether one assumes any event led to it.

However, even in a context where a vase is somehow made to be in a state that otherwise might be called shattered -if an artist prepares numerous ceramic pieces that are carefully sculptured so that they could be assembled into a vase but nonetheless never are-it is difficult to describe the collection of pieces as shattered or a shattered vase, and certainly (16a) does not felicitously describe this situation. Similar contexts for cracked do not improve the readings (though see \$2.2.2 for a potential complication that might affect the interpretation cracked and other verbs in Levin's break class regarding readings of change across space rather than 
time). As for (16c-e) it is difficult for us to even conceive of analogous scenarios where someone may be deemed murdered or something deemed barbecued or cooked when the actions described by murder, barbecue, and cook respectively did not occur, effectively supporting our point that the adjectives themselves entail a change and this explains the contradictoriness of (16c-e). For cooking verbs perhaps the relevant state is something about the relative textural and chemical properties of the food and/or its temperature and edibility, though if (say) a substance is chemically synthesized in a lab to have the relevant properties of barbecued chicken, (16d) still sounds odd. For murdered, if we found a seemingly dead body surrounded by evidence of a violent, externally caused death but in fact the body was stitched together from amputated body parts, then (16e) is not a felicitous description. The same facts all apply to (17) as well. ${ }^{9}$

Finally, this same split in entailment behavior of PC versus result roots has been replicated for other languages (see Jerro 2017b on Kinyarwanda, Valle et al. 2017 on Kakataibo, Spathas 2017 on Greek, and Beavers et al. 2018 on Hebrew and Marathi; we discuss Spathas’s data in \$2.4.1). This suggests that this distinction is not just a matter of English but is something that can be found for the same root classes in other languages as well, even controlling for context. In sum, even beyond verbal contexts result roots but not PC roots give rise to an entailment of change, suggesting that this entailment is part of the root meaning and not introduced templatically despite being one that is canonically considered templatic, namely the entailment introduced by $v_{\text {become }}$ (or other functional heads in other, related frameworks). We now turn to two complications with these judgments that must be taken into account.

\subsubsection{Entailments of change in derived statives}

Notwithstanding judgments like those above, there have been documented in English and other languages what Nedjalkov and Jaxontov (1988) (and papers in that collection) call in their typological survey "derived statives"-deverbal adjectives that fail to entail there was any temporal change giving rise to the described state. ${ }^{10}$ On the surface, such examples look like precisely the kinds of

\footnotetext{
${ }^{9}$ An alternative test might use the active transitive as in The vase is shattered but nobody shattered it, which is acceptable. However, the subject here is human-denoting nobody, yet not only human agents but also events, states, inanimates, and other kinds of entities can cause changes of state events (e.g. The earthquake/the hammer shattered the vase). The reading with nobody is most naturally a denial that it was a human that led to the shattered state, not that there was no shattering event at all, and thus such data do not argue against shattered entailing a change. It is for reasons of avoiding such confounds that we have generally avoided contradictions in which a cause is explicitly expressed, especially when the negation is expressed as a quantifier over the causer as with nobody, for which a denial of causation reading is especially salient.

${ }^{10}$ See also Dubinsky and Simango (1996) for discussion of similar facts in Chichewa and their analytical consequences, which we believe are incorrect given the observations in Koontz-Garboden (2010) and Deo et al. (2011).
} 
data predicted by Bifurcation. Consider (18), where it is clear the lines in question, given the nature of standard writing paper, do not undergo any temporal breaking event to come to be in the state described by broken:

...this paper provides a guide for writing letters that extend below the baseline. Internal broken lines serve as a reference for writing half-space letters. <http://nwccog.co.networkofcare.org/aging/assistive/list.aspx? indexingterms $=$ writing- paper $>($ Accessed May 11 2018)

This might lead one to conclude that result roots, like PC roots, do not actually entail change in their adjectival forms. However, there are several observations to make about such examples, all of which clearly indicate that data such as this do not support this conclusion. ${ }^{11}$

First, there is clear evidence that these adjectives are in fact derived from verbal uses that have the same interpretation, since for each such use there is a corresponding verbal usage that also fails to entail change in time. Consider (19), which is clearly temporally stative given the non-habitual present tense use of the verb (see Dowty 1979: 55-6 for discussion of non-habitual readings of the English present tense as diagnostic of stativity):

(19) ONE suggestion on ur story, it's hard to read when the line suddenly breaks off and $u$ hav to go down a line in the middle of a sentence, to make it flow easier...

<http://www.fanfiction.net/r/6766517/> (Accessed June 2011)

Thus it might not be anything to do with adjectives like broken; it could instead be that the root itself entails no change. However, despite the stativity of (19) it can be shown with straightforward contradiction tests that such uses do entail change, albeit that the change is not temporal in nature.

Rather, as recent work has demonstrated changes of state can also be measured along dimensions other than time, including change measured along space (Gawron 2009, Koontz-Garboden 2010) and even change measured across nontemporal and non-spatial domains such as change across populations of individuals (Deo et al. 2013). This can be seen in (19) from the fact that this example takes an adverbial modifier that selects dynamic predicates, in particular suddenly. The non-temporal change in such verbs can be seen more clearly by examining

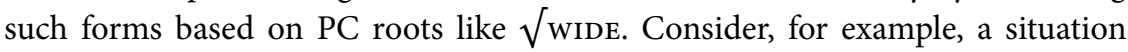

\footnotetext{
${ }^{11}$ Although more work is needed, it seems possible that these observations extend to similar phenomena that have been observed in other languages, e.g. Greek (Alexiadou and Anagnostopoulou 2008) and Hebrew (Doron 2009), though we leave this for future work.
} 
in which a road is constructed so that it is wider at some points than at others. Such a situation can be described with a present tense verb form in English, hence indicating temporal stativity:

(20) I65 widens up ahead.

Notwithstanding the temporal stativity, there is no question that the verb is dynamic, i.e. that it entails of the eventuality it describes that there is change in the road over space. This can be seen from a variety of facts. First, analogous with (19), it can be modified by adverbs like quickly, which can modify only dynamic predicates:

(21) I65 quickly widens up ahead.

Here the reading is that the spatial transition does not take a lot of space. Additionally, when contrasting the deverbal adjective with the morphologically basic one that the verb is derived from a contradiction can be generated with the former but not the latter by denying that there was a change in the eventuality described by the predicate-a standard test for entailment of change (KoontzGarboden 2010, Beavers 2011b; see also Chapter 4):

(22) a. I65 is wide at Lafayette city center. In fact, it's of the same width for its entire extent.

b. \#I65 is widened at Lafayette city center. In fact it's of the same width for its entire extent.

The key observation is that while change-of-state verbs entail change in some domain, it need not be change in time (see Koontz-Garboden 2010, Deo et al. 2013 for further discussion). This leads to the possibility of stative verbs of change of state in both PC and result roots, and thus that adjectives derived from these verbs may have stative change-of-state readings as well.

With this as background, we now return to examples like (18) with result roots. With such roots there is no contrasting morphologically basic adjective counterpart like (22a) (as we noted above). However, it is nevertheless clear that denial of an inference of change in spatial change contexts with result root adjectives (like (18)) leads to contradiction, showing that they entail change even if the change is not temporal. In order to see this, consider the broken line example from (18) in a context where gaps in a line are labeled with letters for ease of reference, as shown using a double line in (23). Crucially, broken can be asserted of a double line if some portions of a double line are preceded by but separated from other portions of the double line by a gap. Thus (23a) is acceptable in such 
a context, given that spatially prior to point $\mathrm{W}$ there is another portion of double line. If we consider a portion of double line at which there is no prior double line, however, as in (23b), the assertion that the double line is broken at that point is sharply infelicitous:

a.

The double line is broken at W.

b. $W=$

\#The double line is broken at W.

Similar examples can be found with others of Levin's (1993: 241-2) break verbs. For example, there is no sense in which the heart in (24) underwent any temporal tearing:

(24) Chesko, 17, was born with a torn valve in his heart. Instead of closing, it flaps, so as the organ pumps, some of the blood returns to the heart rather than leaving it and circulating in the body.

$<$ http://thetimes-tribune.com/sports/h-s-wrestling-valley-view-juniorreturns-to-mat-1.1100620\#axzz1kk0FksF6> (Accessed May 11 2018)

Much as with the break data, however, it does not follow from (24) that torn is not semantically (and morphologically) deverbal. Indeed, there are verbal uses of tear as in (25) that are also clearly stative and also fail to have any entailment of temporal change:

(25) You bread photos are great. Is there a trick in making the cuts so that your bread tears at the cuts like your french loaves and not elsewhere. Many times mine will just open up but flatten out but it [sic] they tear it is on one side near the bottom.

$<$ http://newsgroups.derkeiler.com/Archive/Rec/rec.food.sourdough/200707/msg00145.html> (Accessed May 11 2018)

Just as with the break examples discussed above, while (25) does not entail change in time it does entail change, albeit change in space. The same is true for deverbal adjectival examples like (25), a fact which can be shown with a contradiction test, as in (26):

(26) \#The bread is torn at the cut. In fact, it is in two pieces.

In spite of the fact that there is no temporal tearing in (26), that there is some change from attached to unattached is entailed. 
In sum, while adjectives based on break roots have uses that fail to entail temporal change, all such adjectives entail change along at least some dimension beyond time where change can be measured out. Interestingly, as noted by KoontzGarboden (2010: 299), cooking roots do not allow such uses. Of the data in (27), for example, Koontz-Garboden observes that (27a) is acceptable in a situation where the entire side of beef in the stipulated location undergoes a cooking in time. However, (27b) is unacceptable in a situation where the side of beef has different degrees of cookedness at different spatial points along it, e.g. with the joint being more cooked than the rib at a single point in time. As Koontz-Garboden notes, (27b) is unacceptable in virtue of the use of the present tense verb form, which requires stativity. The verb cook, however, has no temporally stative use like the stative but spatially dynamic uses of other change-of-state verbs illustrated in $(22)-(26):^{12}$

(27) a. The side of beef is cooking between the rib and the joint.

b. \#The side of beef cooks between the rib and the joint.

(Koontz-Garboden 2010: 299)

Cooking verbs (and killing verbs, we also believe) strictly entail temporal change; atemporal change contexts are impossible with them, suggesting that roots can not only entail change but also select for the kind of change they entail. This is an observation whose consequences we will return to below.

\subsubsection{Sources of variation}

A second complication has to do with variation in judgments across speakers and for different roots among the PC and result classes above. For example, with broken in particular, our judgments are that it is unacceptable in the frames in \$2.2.1, suggesting that it entails a change:

(28) a. \#The vase is broken but it didn't break.

b. \#The vase is broken but it has never been broken.

c. \#The vase is broken but it did not undergo a breaking.

Although (28) are contradictory for us, several speakers have pointed out to us that for them broken admits a reading wherein something can be manufactured in a broken state (e.g. predicting that The vase was made broken can have a meaning

${ }^{12}$ Of course, it is possible that a temporal change can give rise to differences across space, as might be the case in (27a) if the temporal change results in different degrees of cookedness across the side of beef. In such a case there are differences across space that are the artifact of a temporal change. But this does not make (27a) a case of spatial change. 
wherein it was manufactured in that state and there was no particular event that led to that state coming about). If so, the prediction is that for such speakers (28) should be acceptable. In fact, we do not rule out the possibility that some English speakers might find them acceptable (and similarly for other result roots). In particular, there are at least two reasons consistent with our analysis for why this might be the case.

First, it is conceivable that change across space or population readings along the lines of those discussed by Deo et al. (2013) for degree achievements as in $\$ 2.2 .2$ might extend to these cases as well (e.g. see the discussion of spatial broken above). A possible reading then is that the vase has some crack or other defect in it as one moves across a spatial dimension, or represents a class of vases across populations of vases going from not broken to broken, but there was no temporal change that led to this. If so, then there is a change here, but the non-temporal change has created a confound.

A second possibility is that there could be a diachronic process of bleaching or other semantic drift that has led to mismatches between morphology and changeof-state entailments, so that at least some adjectives that are superficially deverbal, over time, become fixed, lexicalized expressions while also losing their entailments of change. Such is clearly the case with closed, for example, which does not entail a prior closing, as evidenced by data such as the following:

(29) The door was built in a closed position, but has never undergone a closing.

As far as we are aware closed is an example of a result root in the morphological sense of having only a seemingly deverbal adjective for which there is also wide speaker agreement that the adjective need not entail a change (our judgments as well). Broken in turn may also have undergone lexical drift for some speakers to have a specialized meaning like "not functioning" or "not canonical in a way that suggests defects," which could be an inherent property rather than one derived through a change. Such processes strike us as expected at the level of individual lexemes and may easily lead to some variation in judgments for particular roots (see Dowty 1979: 309-19 for an extended discussion of issues related to lexicalization and lexical drift of this sort in the context of a rule governed system of word formation; see also \$4.1). Furthermore, such lexicalization differences could also exist on a speaker to speaker basis: different people may have slightly different understandings of the relevant terms.

However, what is key for us is that members of the result root group in (3) contain numerous examples of roots where it is hard to see that the adjectival forms ever have readings lacking change (e.g. a speaker who believes broken does not entail a change agrees that other such stative terms seem contradictory when denying a change). What is critical for us is simply the existence of any terms that do entail change among this group, even if not every one does either categorically 
or for all speakers, since our goal is to show that counterexamples to Bifurcation exist, most specifically among result roots. Conversely, few if any basic PC root adjectives have entailments of change that we are aware of, though if any did this could also be accounted for through idiosyncratic lexical drift. In sum, we expect speaker-to-speaker and item-by-item variation, though ultimately the existence of any forms defying Bifurcation for any set of speakers is sufficient to demonstrate our point that Bifurcation does not universally hold. ${ }^{13}$

\subsubsection{Summary: Predictions of Bifurcation and semantic explananda}

The main conclusion from the discussion above is that there is no context in which entailments of change fail to surface with result roots. Even derived stative contexts entail that the participant the state holds of underwent some change, though it need not have been a temporal change. Furthermore, the verbs that result root adjectives are derivationally related to have these same uses. This entailment of change (temporal or otherwise) distinguishes result roots from PC roots, which are clearly found (in their morphologically simple form) without a change entailment of any kind-inferences of temporal and atemporal change can be readily denied with PC roots in their morphologically simple form without giving rise to contradiction. The fact that many result roots generally cannot be disembodied from an entailment of change in any context, while PC roots can, suggests that the former by contrast with the latter have this as part of their root meaning (modulo some speaker-to-speaker and root-by-root variation). Furthermore, we have observed that roots can select for particular kinds of change, with some roots, like crack, break, etc., underspecified for the type of change, while cooking and killing roots lexically specify for temporal change only.

\footnotetext{
13 The claim that some roots entail change does not preclude "fun with language" examples where no change has occurred. These are cases in which some object is described as a thing that it is meant to resemble. For example, a reviewer points to Massimo Bottura's recipe "Oops! I dropped the lemon tart" $<$ http://www.epicurious.com/recipes/food/views/oops-i-dropped-the-lemon-tart-51198010>, for a dessert that is meant to look like a lemon tart that has been dropped, broken up, and is a mess on the plate. That the dessert has not undergone a dropping event in space, time, or presumably any dimension is irrelevant, we believe, as this is simply a name, much in the way a work of art might have a name evoking what the art is meant to represent. Thus a sculpture might be inspired by a storm on a hill and be given the name "Storm on a hill" by the artist creating it, even though it is clearly neither a storm nor a hill. We believe this dessert case is parallel, in that it is arguably neither a tart nor certainly dropped, but rather inspired by what a real dropped lemon tart might look like. Such fun with language is entirely normal, but irrelevant for the matters at hand.
} 
This gives rise to the semantic typology in (30), which any theory of the syntax/semantics of change-of-state verbs must find a way of capturing:

\begin{tabular}{l|l} 
Root type & Change entailments \\
\hline PC roots & $\begin{array}{l}\text { no change entailed } \\
\text { change of any kind entailed } \\
\text { break-roots } \\
\text { cook-and kill-roots }\end{array}$
\end{tabular}

This typology (and the more basic one of PC vs. result roots) in turn leads to a predictive typology of verbs based on these different roots, something that is crucially rooted solely in differences in root meaning and nothing to do with the templates these roots occur in. In $\$ \$ 2.4-2.5$, we return to the typology in (30) to consider its consequences for Bifurcation and the architecture of an adequate theory of change-of-state verbs. First, however, we consider what Bifurcation entails for the morphological shape of change-of-state verbs and derivationally related words.

\subsection{Morphological consequences of Bifurcation}

\subsubsection{Four morphological predictions}

Bifurcation in the context of change-of-state verbs also predicts that, modulo language and root-specific morphological accidents, words derived from the roots of all change-of-state verbs should be identical in morphological complexity. This is because what defines change-of-state verbs is the presence of the same templatic operators in the same arrangement, and these operators should in the general case have the same exponence across all roots. Consider, for example, the Embick-style structures for basic adjectives, result state adjectives, inchoatives, and causatives introduced above and repeated in (31):

(31) a. Basic states (e.g. adjectives, statives, etc.)

(Embick 2004: 363)

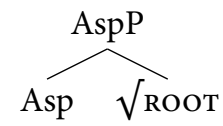

b. Result states (Embick 2004: 367)

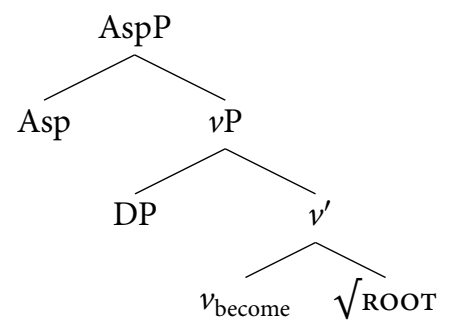


c. Inchoatives (Embick 2004: 365; see also $\$ 1.3 .1,(21 b)$ )

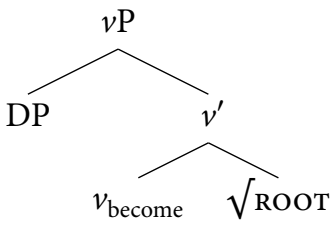

d. Causatives (Embick 2004: 366; see also \$1.3.1, (21c))

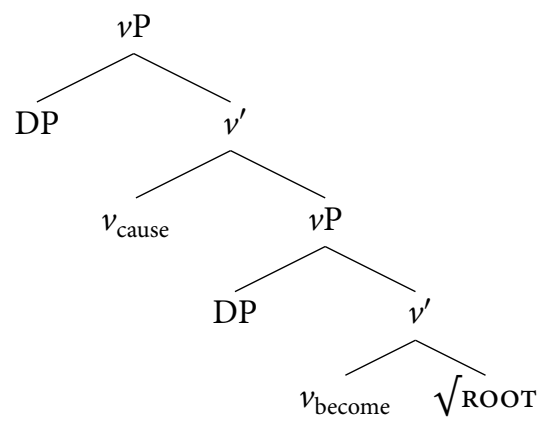

Since all adjectives, inchoatives, and causatives are formed in the same way, with the same operators in the same arrangement, then all things being equal these operators should have the same realization across root classes. For example, internal to a single language $v_{\text {become }}$ should be realized the same way (or perhaps in one way for inchoatives, one way for causatives, and one way for deverbal adjectives), Asp the same way (or at least in two different ways), and similarly for all templatic operators. In short, internal to a single language all inchoative and causative change-of-state verbs should be identical in morphological complexity and overt form, as should their corresponding adjectives, since what makes any root into an adjective, inchoative, or causative is the same templatic operator/functional head across all roots of change-of-state verbs. These predictions are articulated in (32):

(32) a. Basic adjectives from all roots have the same complexity and morphological forms

b. Result adjectives from all roots have the same complexity and morphological forms

c. Inchoative change-of-state verbs derived from all roots have the same complexity and morphological forms

d. Causative change-of-state verbs derived from all roots have the same complexity and morphological forms

This does not preclude the possibility that there are principled patterns of subregularity. For example, verbs of personal grooming such as shave or dress are arguably change-of-state verbs, yet tend to form special morphological subclasses in many 
languages (as middle voice verbs, often marked as reflexive in languages; see e.g. Kemmer 1993: 54-5), and we might expect other types of principled subregularity rooted in semantics (e.g. see also potential morphological and syntactic distinctions between verbs based on the internal vs. external caused distinction of Levin and Rappaport Hovav 1995: 91 or the agent-subject vs. causer-subject distinction as per Van Valin and Wilkins 1996 and Koontz-Garboden 2009a, inter alia).

However, if we assume Bifurcation, then the PC vs. result root distinction as a fact about semantics wherein the former roots do not entail a change and the latter do is not a possible distinction. Thus absent any other way of distinguishing these two root classes (some such proposals for which we discuss and dismiss in \$2.5) the expectation is that the roots in these two classes should in the general case have the same morphological properties. Of course, within any (sub)class of words the morphology for any individual item may be idiosyncratic and exceptional owing to factors such as historical accidents, and thus there could be quite a lot of individual variation. ${ }^{14}$ However, it should be the case that any such deviation within a given root (sub)class should be random. Thus to the extent that PC and result roots show distinct, recurring morphological distinctions Bifurcation is called into question. Whether this is true in relation to $(32 \mathrm{c}, \mathrm{d})$ remains a somewhat open question, though preliminary data from Eastern Armenian (Megerdoomian 2002), Hebrew (Doron 2003), Greek (Alexiadou and Anagnostopoulou 2004), and Ulwa (KoontzGarboden 2006) all suggest that PC roots and result roots do indeed differ in their morphological marking in the causative alternation, at least in those languages. This question is investigated in a more systematic way in Beavers et al. (2018), drawing on the same crosslinguistic sample described further below. The results there point in the direction of falsification for $(32 c, d)$. We do not deal with these predictions further here, referring the interested reader instead to Beavers et al. (2018). Instead we focus in more detail on (32a,b).

\subsubsection{Adjectives derived from the different root classes across languages}

Contrary to the prediction of Bifurcation, as already seen above adjectives derived from the roots of English change-of-state verbs are not morphologically homogeneous. While there are two kinds of adjectives derived from PC roots (morphologically basic and deverbal), from result roots there is only one, which superficially seems to be deverbal (and behaves that way semantically; see $\$ 2.2$ ). Any the-

\footnotetext{
14 Alternatively, morphological differences among verbs may be conditioned purely by phonology, though for the moment we set this aside since such facts would be language particular and for English there is no obvious consistent phonological distinction between result and PC roots.
} 
ory preserving Bifurcation must treat the English situation as a morphological accident, since, again, the same templatic operators are at play with the roots of all change-of-state verbs. This is precisely what Embick (2004: 363) proposes. Specifically, Embick claims that in English, adjectivalizing morphology (-ed and its allomorphs) realizes the Asp head in both (4) and (5) with result roots, while Asp with PC roots is realized differently in the two structures-null in (4) and overt in (5). The contrast between the two root types is thus an accident of English morphology.

This is in principle a plausible account of English-it just happens that what we are calling PC roots are those that fall into one morphological class and what we are calling result roots are those that fall into another. However, there are two complications with this. First, the morphological accident happens to correlate with a recurring semantic difference discussed in $\$ 2.2 .1$, where basic PC root adjectives systematically lack change-of-state entailments but categorically marked result root adjectives often entail change. This suggests that the morphological distinction might be tied to other properties of these roots. Furthermore, since morphological accidents are by definition language- and root-specific (see discussion and references in Koontz-Garboden 2016: 96-7) such an analysis predicts that the same kind of morphological split between PC and result roots should not repeat itself in other languages. As it turns out, there have been various isolated observations in unrelated languages of morphological contrasts of just this type in prior literature. For example, the data in (33) from Megerdoomian (2002) show that just like English, many Eastern Armenian roots lack an adjective of the same kind of morphological form as that describing the basic state for $\mathrm{PC}$ roots:

a. Eastern Armenian PC verbs (Megerdoomian 2002: 98)

\begin{tabular}{lll} 
adjective & non-causative COS & causative COS \\
\hline $\begin{array}{l}\text { layn 'wide' } \\
\text { čor 'dry' }\end{array}$ & layn.anal 'widen' & layn.ats.nel 'widen' \\
metz 'big' & čor.anal 'dry' & čor.ats.nel 'dry' \\
arag 'fast, quick' & arag.anal 'grow' & metz.ats.nel 'grow, bring up' \\
čaq 'fat' & čaq.anal 'become fat' & arag.ats.nel 'accelerate' \\
sev 'black' & sev.anal 'blacken' & sev.ats.nel 'fatten'
\end{tabular}

b. Eastern Armenian result verbs (Megerdoomian 2002: 98)

\begin{tabular}{lll} 
adjective & causative COS & non-causative COS \\
\hline- & k'ot'Rel 'break' & k'ot'R.v.el 'break' \\
- & epel 'cook' & ep.v.el 'cook' \\
- & poxel 'change' & pox.v.el 'change' \\
- & xort'ak'el 'sink, drown' & xort'ak'.v.el 'sink, drown'
\end{tabular}


Koontz-Garboden (2006, 2009b, fieldnotes) notes a similar contrast in Ulwa:

Ulwa (Koontz-Garboden 2006; fieldnotes)

a. "deadjectival" verbs

\begin{tabular}{lll} 
state & non-causative COS & causative COS \\
\hline auh- 'fat' & auh-ta- 'fatten' & auh-ta- 'fatten' \\
pau- 'red' & pau-ta- 'redden' & pau-ta-'redden' \\
yam- 'good' & yam-pa- 'become better' & $?$ \\
dut- 'bad' & dut-ta- 'get worse' & $?$
\end{tabular}

b. break verbs (unmarked meaning of root in -ta/pa-; Koontz-Garboden 2009b)

\begin{tabular}{lll} 
state & non-causative COS & causative COS \\
\hline$*$ & bah-wa- 'break (intrans)' & bah-ta- 'break (trans)' \\
$*$ & lah-wa- 'boil (intrans)' & lah-ta- 'boil (trans)' \\
$*$ & birh-da- 'tear (intrans)' & birh-pa- 'tear (trans)' \\
$*$ & bis-da- 'rip (intrans)' & bis-pa- 'rip (trans)'
\end{tabular}

Similar data can be observed in Pima (Smith 2006: 3), Tongan (Koontz-Garboden 2005), O’odham (Hale and Keyser 1998: 92), Kakataibo (Valle et al. 2017), and Kinyarwanda (Jerro 2017b). Thus a PC vs. result root split is attested beyond just English, suggesting the distinction might be linguistically significant. That said, convenience data from a few languages, while suggestive, do not amount to a fully systematic investigation.

However, in recent work Beavers et al. (2018) show beyond doubt that the prediction of Bifurcation that there should be basic adjectives based on both root types is false. ${ }^{15}$ Using a combination of dictionary and grammar mining plus some native speaker work (building on methodologies used by Croft 1990, Haspelmath 1993, and Nichols et al. 2004), Beavers et al. collected paradigms of basic stative, inchoative, causative, and result stative forms (e.g. red-redden-redden-reddened) for 72 total root meanings, 36 PC root meanings (drawing on Dixon's (1982: 16) major property concepts) and 36 result root meanings (drawing on Levin's (1993) non-deadjectival English verb classes) in a balanced sample of 88 languages largely drawn from the WALS-100 list (Comrie et al. 2013). The relevant root meanings are given in (35) and (36) (as an adjective or verb, with synonyms or hypernyms considered in that study given in parentheses) (Beavers et al. 2018: (3)-(4)):

\section{(35) Property Concept Roots}

a. Dimension: large/big/enlarge, small/shrink/shrunken, short/shorten, long/lengthen, deep/deepen, wide/widen, tall/height/heighten

15 A preliminary version of the study is reported in Beavers et al. (2017). 
b. Age: old/aged/age

c. Value: bad/worsen/worse, good/improve/improved

d. Color: white/whiten, black/blacken, red/redden, green/make green, blue/make blue, brown/make brown

e. Physical Property: cool/cool, cold/make cold, warm/warm, hot/heat up, dirty/dirty, dry/dry, wet/wetted, straight/straighten, hard/harden (tough/toughen), soft/soften, tight/tighten, clear/clear, clean/clean, smooth/smooth, sharp/sharpen, sweet/sweeten, weak/weaken, strong/ strengthen

f. Speed: fast/speed up, slow/slow down

(36) Result Roots

a. Entity-specific Change of State: burned/burn, melted/melt, frozen/freeze, decayed/decay (rotten/rot), swollen/swell, grown/grow, bloomed/bloom (flowered/flower, blossomed/blossom), withered/wither (wilted/wilt), fermented/ferment, sprouted/sprout (germinated/germinate), rusted/rust, tarnished/tarnish

b. Cooking Verbs: cooked/cook (baked/bake, fried/fry, roasted/roast, steamed/steam), boiled/boil

c. Breaking Verbs: broken/break, cracked/crack, crushed/crush, shattered/shatter, split/split, torn/tear (ripped/rip), snapped/snap

d. Bending Verbs: bent/bend, folded/fold, wrinkled/wrinkle (creased/crease)

e. Verbs of Killing: dead/killed/kill, murdered/murder, drowned/drown

f. Destroying Verbs: destroyed/destroy (ruined/ruin)

g. Verbs of Calibratable Change of State: go up (raised/rise, ascended/ascend, increased/increase, gained/gain), go down (fallen/fall, dropped/drop, descended/descend, decreased/decrease, declined/decline), differ/ different

h. Verbs of Inherently Directed Motion: come/came, gone/go, go in (entered/enter), go out (exited/exit), returned/return

Beavers et al. (2018) demonstrate a robust difference already suggested by the preliminary data observed in previous language-specific studies discussed above: while PC root meanings are overwhelmingly associated with basic stative forms (nearly all PC roots where at least one member of the paradigm was attested had an attested basic stative form), there are rarely basic stative forms associated with result root meanings (almost no result roots where at least one member of the paradigm was attested had an attested basic stative form), a statistically significant 
result. ${ }^{16}$ Taken together the evidence from the language particular studies cited above and the broader typological study of Beavers et al. (2018) suggest that there is a general crosslinguistic tendency for PC roots and result roots to differ in morphological ways that is not consistent with a Bifurcation.

\subsubsection{Summary: Predictions of Bifurcation and morphological explananda}

Contrary to the prediction of Bifurcation, there seems to be a systematic contrast between PC roots and result roots in that state-denoting words based on result roots do not exist in the same morphological forms as those based on PC roots. In short, the Bifurcation Thesis misses a significant morphological generalization. It predicts that any difference in the morphological shape of state-denoting words derived from PC roots and result roots should be purely accidental. The crosslinguistic repetition of the pattern shows unambiguously, however, that it is not. This suggests that the distinction is linguistically significant, and combined with the correlating semantic observation discussed above strongly suggests that the relevant basis for the distinction is that $\mathrm{PC}$ roots do not entail change but result roots do, contra Bifurcation.

\subsection{Analytical option 1: Abandon Bifurcation}

In the preceding sections we examined semantic and morphological predictions of Bifurcation in the domain of change-of-state verbs. Contrary to the predictions that all change-of-state verbs should behave identically in both domains, we have observed significant contrasts between subclasses of such verbs. First, while PC roots can be disembodied from an entailment of change, result roots never can be. Second, result roots contrast among themselves in the kinds of change they lexically entail. Third, result roots and PC roots contrast in the morphological contexts in which they can appear. We now consider the consequences of these observations for the Bifurcation Thesis and the kinds of analyses these facts motivate. We begin with analyses that would break with Bifurcation, as we believe the facts necessitate. We then turn to a discussion of how it could be maintained and the empirical and theoretical consequences of doing so.

\footnotetext{
16 There are some noteworthy counterexamples around the edges discussed by Beavers et al. (2018), which ultimately have explanations consistent with the overall observations. Beavers et al. furthermore show that the statistical results hold up even controlling for various issues that arise in typological studies of this sort owing to data spareseness, e.g. controlling for the possibilities of translation bias that might affect how stative terms are coded and for lack of attested terms in lexical resources in the face of known productive processes for deriving terms of the appropriate sort.
} 


\subsubsection{A purely non-lexicalist analysis}

As we discussed in $\$ 1.3$, much recent work on verbs at the syntax/semantics interface is conducted in the context of various non-lexicalist theories, including in particular Distributed Morphology (DM), in which Bifurcation was originally couched. However, the two can certainly be decoupled, so that an analysis in which all word formation is syntactic and based on acategorial roots is possible to maintain while still rejecting Bifurcation. In this section we develop the outlines of such an analysis.

The starting point is precisely the assumption that violates Bifurcation-that at least some result roots come with an entailment of change, while PC roots do not. In its most straightforward formulation, this would be to assume that a root like

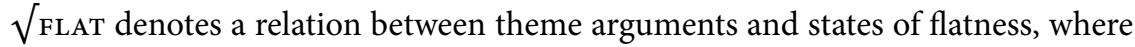
the flat' relation does not have as part of its truth conditional content anything

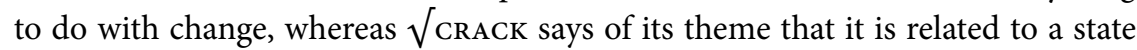
$s$ of having some fissure in its material integrity-for simplicity we represent this as a state cracked', though this is just a mnemonic logical symbol and not meant to be equated with the English participle-and furthermore requires that there be some eventuality $e^{\prime}$ that led to this state of affairs. To capture that both spatial and temporal change readings are possible we assume (as already mentioned in $\$ 1.3 .2$, fn. 8) that become' may take either a proper event as the eventuality of change, which would result in temporal change, or a state, in which case the change occurs over a spatial region or a population: ${ }^{17}$
a. $\llbracket \sqrt{\text { FLAT }} \rrbracket=\lambda x \lambda s\left[\right.$ flat $\left.^{\prime}(x, s)\right]$
$(=(22 \mathrm{a})$ in $\$ 1.3 .2)$
b. $\llbracket \sqrt{ }$ CRACK $\rrbracket=\lambda x \lambda s\left[\operatorname{cracked}^{\prime}(x, s) \wedge \exists e^{\prime}\left[\operatorname{become} e^{\prime}\left(s, e^{\prime}\right)\right]\right]$

In reality the existence of an entailment of change is probably best thought of as being part and parcel with what it means to be in the state that cracked describes in the appropriate sense, i.e. it is a fact about how we conceive of the kinds of states that such result roots describe independent of their linguistic expression and thus should follow as part of the truth conditions imposed by the formative cracked' that defines the idiosyncratic contribution of this root (e.g. $\forall x \forall s\left[\operatorname{cracked}^{\prime}(x, s)\right.$

\footnotetext{
17 This kind of analysis presupposes that atemporal dynamic eventualities are states, as evidenced by their temporal stativity, as discussed above (see also Kratzer 2000: 392-3 for a similar observation). See Gawron (2009) and Koontz-Garboden (2010) for discussion of how atemporal change upends the traditional event/state dichotomy. That said, what we are proposing here is not meant to be a fullyfledged analysis of the temporal vs. atemporal change distinction, which is beyond the scope of the

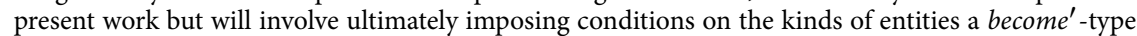
relation entails that the change described by the verb occurs along and what that relationship is (e.g. a Figure/Path Relation as for events of change as in $\$ 1.6 .1$, or something else; see Deo et al. 2013 for a more fleshed out theory). However, the details will not matter here and thus we adopt this simplification as a placeholder.
} 
$\left.\left.\rightarrow \exists e^{\prime}\left[\operatorname{become}^{\prime}\left(s, e^{\prime}\right)\right]\right]\right)$. As such it need not be listed separately per se. However, for clarity here and below we continue to represent it as a separate part of the meaning of the root so as to make the contrast with PC roots clearer. (See $\$ 5.2$ for further discussion. $)^{18}$

Assigning the change-of-state head $v_{\text {become }}$ has a denotation as in (38) and combining it with the roots in (37) derives the inchoative predicates for the contrasting root classes in $(39 \mathrm{a}, \mathrm{b})$ :

$$
\llbracket v_{\text {become }} \rrbracket=\lambda P \lambda x \lambda e \exists s\left[\operatorname{become}^{\prime}(s, e) \wedge P(x, s)\right]
$$

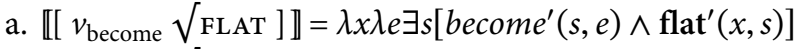

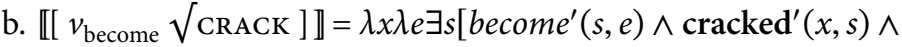 $\left.\exists e^{\prime}\left[\operatorname{become} e^{\prime}\left(s, e^{\prime}\right)\right]\right]$

The formulae $(39 a, b)$ are identical in their lexical entailments save for the final state. But for result roots the root itself already lexically entails the existence of the change independent of $v_{\text {become }}$. It is not problematic that $v_{\text {become }}$ and $\sqrt{\text { CRACK }}$ both introduce an entailment of change. The relevant meaning components are conjoined and thus though they are introduced separately the result is truthconditionally equivalent to change being introduced just once. By contrast, such an entailment is only found in words built on PC roots when $v_{\text {become }}$ is first composed with the root. In either case, when combined with $v_{\text {become }}$ the two resulting verbal forms are identical in entailing change.

This analysis makes two key semantic predictions about where the roots diverge. First, it predicts there can be no adjective formed with a result root lacking the entailment of change, in contrast with a PC root. This prediction, which is borne

\footnotetext{
18 This analysis bears some similarities to Kratzer's (2000) analysis of German stative participle formation. In particular, she also proposes (pp. 390-1) a class of acategorial roots that entail both a state and change, the ones in German that form target state participles (e.g. auf-pumpen 'pump up'), where the acategorial status is required to generate the target state participles since the relevant stativizer only applies to acategorial roots. Thus these roots are akin to our result root denotations. However, she also posits another class of adjective/verb paradigms that do not form such participles, which she proposes are formed from lexicalized adjectives (e.g. leer(en) 'empty $\mathrm{Adj}_{\mathrm{V}}$ ') with subsequent possible verbalization, which will not form target state participles since there is no acategorial root (pp. 393-4). In this sense there is a lack of decompositional structure to accomplishments based on these, which she says yields an inability for the latter class to take sublexical modifiers like für 'for' PPs modifying the underlying state.

However, Kratzer does not make any claims about whether any of these adjectives have event entailments or not, and thus it does not correlate semantically with our PC roots. Furthermore, in English both PC and result roots can show the same sublexical modification facts as with for an hour (e.g. PC root John emptied the basin for an hour and result root John dropped his head for five minutes, though factors like reversibility of the state may matter for acceptability as per $\$ 1.3 .2$ ), meaning there is no formal distinction between them of the sort Kratzer claims separates her auf-pumpen and leeren classes. For this reason our own analysis treats PC and result roots as formally identical in semantic type and category (both being acategorial roots denoting functions over individuals and states, though acategorialness is, as noted in $\$ 1.3$, not an important feature of our analysis), differing only in entailments.
} 
out by the data already discussed in $\$ 2.2$, can be seen in the simple derivations in (40) for adjectives like those based on the PC root $\sqrt{\text { FLAT in }}(40 \mathrm{~b})$ and the result

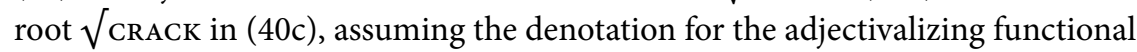
head is an identity function in (40a):
a. $\llbracket$ Asp $\rrbracket=\lambda P[P]$
b. $\llbracket[$ Asp $\sqrt{\text { FLAT }}] \rrbracket=\lambda x \lambda s\left[\right.$ flat $\left.^{\prime}(x, s)\right]$
c. $\llbracket\left[\operatorname{Asp} \sqrt{\text { CRACK }] \rrbracket}=\lambda x \lambda s\left[\operatorname{cracked}^{\prime}(x, s) \wedge \exists e^{\prime}\left[\operatorname{become}^{\prime}\left(s, e^{\prime}\right)\right]\right]\right.$
$(=\$ 1.6 .1 .3,(49 a))$

The derivation in (40c), then, shows that we can generate the right meaning for result roots in basic adjectival contexts if we simply dispense with Bifurcation and admit that there is an entailment of change in the root.

A second prediction of this analysis concerns the nature of the change in result roots. If it is really a lexical property of some roots that they have an entailment of change, then we might expect the nature of that change to differ across roots, with some roots selecting for one kind of change, and other roots selecting for another. Precisely this state of affairs is reflected by the typology laid out in (30) and by the data showing that while some result roots, e.g. the break ones, are underspecified for the nature of the change they lexically entail and thus allow both temporal and atemporal changes, others, like the cooking verbs, can only describe temporal change. This contrast can be captured by assuming that the nature of the change

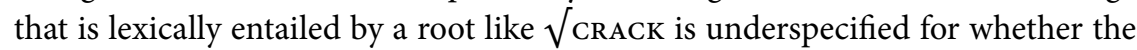
causing eventuality is a state or an event as in (37b), while the change in cooking roots is lexically restricted to be temporal by virtue of constraining any eventuality giving rise to the change to be an event as opposed to a state, i.e. any such causing event $e^{\prime \prime}$ is in the set of events $U_{E}$ as opposed to the set of states $U_{S}$ :

$$
\begin{aligned}
& \llbracket \sqrt{ } \operatorname{cook} \rrbracket=\lambda x \lambda s\left[\operatorname{cooked}^{\prime}(x, s) \wedge \exists e^{\prime}\left[\operatorname{become} e^{\prime}\left(s, e^{\prime}\right)\right] \wedge\right. \\
& \left.\forall e^{\prime \prime \prime \prime}\left[\operatorname{become} e^{\prime}\left(s, e^{\prime \prime \prime \prime}\right) \rightarrow e^{\prime \prime \prime \prime} \in U_{E}\right]\right]
\end{aligned}
$$

A third prediction concerns the scope of modification with adverbial modifiers like again discussed in $\$ 1.3 .2$. Recapping, with change-of-state verbs like open there are two distinct readings that again can have (Dowty 1979: 252): the restitutive reading wherein only the state had occurred previously but not necessarily a change into that state, and the repetitive reading wherein the (caused) change of state had occurred before, something that supposedly follows from a scopal ambiguity of sentence final again as in (42): ${ }^{19}$

\footnotetext{
${ }^{19}$ For illustrative purposes we assume repetitive modification is attachment to the highest $v \mathrm{P}$, but any attachment site above the root will generate a repetitive reading of some sort, with different attachment sites differing in what else beyond the state occurred previously (as per \$1.3.2).
} 
(42) Kim opened the door again.

a. $\left[{ }_{v \mathrm{P}} \operatorname{Kim}\left[{ }_{v^{\prime}} v_{\text {cause }}\left[{ }_{v \mathrm{P}}\right.\right.\right.$ the door $\left.\left.\left.\left[{ }_{v^{\prime}} v_{\text {become }}[\sqrt{\text { OPEN again }}]\right]\right]\right]\right]$

b. $\left[{ }_{v \mathrm{P}}\left[{ }_{\mathrm{P}} \mathrm{K} \operatorname{Kim}\left[{ }_{v^{\prime}} v_{\text {cause }}\left[{ }_{v \mathrm{P}}\right.\right.\right.\right.$ the door $\left.\left.\left[{ }_{v^{\prime}} v_{\text {become }} \sqrt{\text { OPEN }}\right]\right]\right]$ again $\left.]\right]$

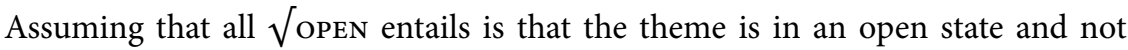
necessarily that it came to be in an open state-in other words that $\sqrt{\text { OPEN }}$ is a PC root with denotation (43a) - and that again has the denotation in (43b), then composing (43b) with (43a) produces $(43 \mathrm{c})$ a relation between open states and individuals $z$ where there had been a prior open state for $z$ :

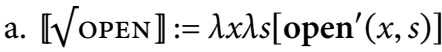
b. 【again $\rrbracket=\lambda P \lambda z \lambda e^{\prime \prime \prime}\left[P\left(z, e^{\prime \prime \prime}\right) \wedge \partial \exists e^{\prime \prime}\left[e^{\prime \prime} \ll e^{\prime \prime \prime} \wedge P\left(z, e^{\prime \prime}\right)\right]\right]$ $(=(26)$ in $\$ 1.3 .2)$

c. $\llbracket[\sqrt{\text { open again }]}]:=\lambda z \lambda e^{\prime \prime \prime}\left[\operatorname{open}^{\prime}\left(z, e^{\prime \prime \prime}\right) \wedge \partial \exists e^{\prime \prime}\left[e^{\prime \prime} \ll e^{\prime \prime \prime} \wedge \operatorname{open}^{\prime}\left(z, e^{\prime \prime}\right)\right]\right]$

Conversely, if result roots such as the one in melt have an entailment of change as part of their meaning then even on low restitutive attachment as in (44a) (parallel to (42b)) there should still be an entailment of change under the scope of again and thus the reading will be repetitive:

(44) Kim melted the ice cream again.

a. $\left[{ }_{v \mathrm{P}} \operatorname{Kim}\left[{ }_{v^{\prime}} v_{\text {cause }}\left[{ }_{v \mathrm{P}}\right.\right.\right.$ the ice cream $\left.\left.\left.\left[{ }_{v^{\prime}} v_{\text {become }}[\operatorname{again} \sqrt{\text { MELT }}]\right]\right]\right]\right]$

b. $\left[{ }_{v \mathrm{P}}\left[{ }_{v \mathrm{P}} \operatorname{Kim}\left[v_{v^{\prime}} v_{\text {cause }}\left[{ }_{v \mathrm{P}}\right.\right.\right.\right.$ the ice cream $\left.\left.\left[{ }_{v^{\prime}} v_{\text {become }} \sqrt{\text { MELT }}\right]\right]\right]$ again $\left.]\right]$

More precisely, if the root of melt has the denotation in (45a) then the restitutive scope reading of again will produce the meaning in (45b) by which not only does the melted state obtain again but the change into it does as well:
a. $\llbracket \sqrt{\text { MELT } \rrbracket}=\lambda x \lambda s\left[\operatorname{melted}^{\prime}(x, s) \wedge \exists e^{\prime}\left[\operatorname{become}\left(s, e^{\prime}\right)\right]\right]$

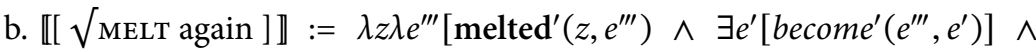 $\left.\partial \exists e^{\prime \prime}\left[e^{\prime \prime} \ll e^{\prime \prime \prime} \wedge \operatorname{melted}^{\prime}\left(z, e^{\prime \prime}\right) \wedge \exists e^{\prime}\left[\operatorname{become} e^{\prime}\left(e^{\prime \prime}, e^{\prime}\right)\right]\right]\right]$

Furthermore, since all higher attachments (e.g. as in (44b)) will likewise scope over an entailment of change (introduced both by the root and also the $v_{\text {become }}$ head), the prediction is that result roots will never admit truly restitutive readings in the truth conditional sense. Precisely this state of affairs has been observed by Rappaport Hovav (2010: 7), who shows that while sentences like (46) with PC roots can be true even if there has been only one change into the relevant state as in the given contexts, with result roots as in (47) the only reading is that there were two events of the relevant state coming about. Thus in contexts in which the 
only possible reading would be a purely restitutive one such predicates should be unacceptable under again modification. ${ }^{20}$

(46) a. [ John buys a knife that was made by a process by which it was forged already sharp. John uses it until it becomes blunt. He uses a whetting stone to sharpen it. ]

John sharpened the knife again. ～(could be just one sharpening)

b. [ A film producer makes a four-hour long film, which is significantly longer than the norm. She is pressured to reduce its length, so cuts it to be two hours. But then the director and actors protest, so she restores it to four hours. ]

The producer lengthened the film again.

(could be just one lengthening)

c. [ Kim takes a photo that is too large to use as a Facebook profile photo. She shrinks it to a more appropriate size, but thinks it does not look good. So she restores it to its original size and puts it on her personal website instead. ]

Kim enlarged the photograph again. (could be just one enlarging)

(47) a. [ A boutique store makes their shirts in the back. Sandy buys one and leaves with it, but then decides she does not want it. She goes back to the store with the shirt and exchanges it. ]

\#Sandy returned the shirt again.

(necessarily two returnings)

b. [ Leah kills a rabbit, takes it home and skins and butchers it and then puts the fresh meat in the freezer for three days. She then takes it out and puts it on the table to thaw. ]

\#Leah thawed the meat again. (necessarily two defrostings)

c. [ An ice cream factory manufactures ice cream from a package of ingredients by adding water and then freezing the result. After adding the contents of the package to water and freezing it, Kim lets it melt into a liquid state. ]

\#Kim melted the ice cream again.

(necessarily two defrostings)

None of the result roots allow a restitutive reading even in contexts that should support one. ${ }^{21}$ Indeed, as discussed in $\$ 2.2 .1$ in many cases with such roots it is

\footnotetext{
${ }^{20}$ In Chapter 4 we make similar observations about manner of killing verbs in that there is no reading with modifiers like again that does not also entail some prior manner by which the change came about, suggesting that these roots violate Bifurcation in a different way, in entailing not only change, but also a cause bringing it about by virtue of a certain sort of action.

${ }^{21}$ These judgments may be subject again to some speaker variation. Although the majority of people we have asked, including ourselves, find examples like (47) all unacceptable, one reviewer remarks that
} 
hard to say what the prior state would be absent a change into that state (a fact which we believe is prima facie evidence for our claim in $\$ 5.2$ that in many cases these states simply do not exist). It is hard to know, for example, exactly what the underlying state of most cooking verbs is without reference to the process. But if we assume the output of fry for example is being warm with brown, fatty edges, then a food item that grows naturally in such a state and comes to lose those properties still does not permit a restitutive reading with again:

(48) [ John is hiking in a very hot region and comes across a fruit that has brown, fatty edges. He picks it and takes it back to his house where he trims off the edges and puts it in the fridge. He later takes it out and fries it. ]

\#John fried the fruit again.

(necessarily two fryings)

The lack of restitutive readings with again thus supports the analysis proposed here which simply has it that a root can have an entailment of change as part of its truth conditional content, contra Bifurcation.

This observation is reinforced by similar observations tied to re-prefixation, and the readings of change-of-state verbs under it. Unlike again, which has been argued to show a genuine syntactically driven attachment ambiguity with high attachment giving rise to the repetitive reading and low attachment giving rise to the restitutive reading, re- has been argued to only attach low (Dowty 1979: 256; Wechsler 1989; Marantz 2007, 2009). The reason for this is that unlike again, which in some positions such as sentence initial and preverbal positions, generates only repetitive readings for all types of verbs, including even PC root change-of-state verbs, $r$ - supposedly always allows restitutive readings in addition to repetitive readings:

a. Again, John opened the door.

(repetitive only)

b. John again opened the door.

(repetitive only)

c. John reopened the door.

(restitutive or repetitive)

while (47a) is indeed unacceptable, she finds (47b) and (47c) acceptable. This may reflect differences in lexicalization across speakers as per $\$ 2.2 .3$. Finally, a reviewer suggests that he finds $(47 \mathrm{a})$ and $(47 \mathrm{~b})$ unacceptable even in repetitive contexts. We disagree, and note that naturally occurring examples can be found:

(i) a. I ordered a pair of shoes in early December... I tried them on and within minutes knew they were the wrong size, so I immediately got online and printed a return shipping label. I ordered the bigger size, which arrived 2 days later, and the next day I took the return package to UPS (Staples) to send the first pair of shoes back... Fast forward a couple weeks, and now I am returning the bigger pair of shoes to Amazon ... I hate to have to return the shoes again. <http://storiesbystephen.com/2017/12/29/ christmas-returns-amazon-vs-foot-locker/> (Accessed June 24 2019)

b. [In response to the question "Why exactly can't you refrost food that has been thawed?"] When you thaw the meat again the second time...

<https://qc.answers.yahoo.com/question/index?qid=20090123002217AAFT7YC>

(Accessed June 24 2019) 
The categorical low scoping analysis of $r e$ - predicts this universal ambiguity between restitutive and repetitive readings by assuming that technically the restitutive reading is the only possible reading, and that any repetitive reading arises from the restitutive reading in pragmatic contexts where repetition has occurred in addition to restitution (since repetition asymmetrically entails restitution). However, crucially, with re-, just as with again, we see a contrast in readings with $\mathrm{PC}$ verbs having genuine restitutive readings and result verbs having only the repetitive reading, in particular by the fact that while the repetitive reading can be denied with PC verbs prefixed by re-, as in (50), it cannot with result verbs prefixed by re-, as shown by (51), even in plausible contexts:

(50) a. [ John buys a knife that was made by a process by which it was forged already sharp. John uses it until it becomes blunt. He uses a whetting stone to sharpen it. ]

John resharpened the knife. (could be just one sharpening)

b. [ A film producer makes a four-hour long film, which is significantly longer than the norm. She is pressured to reduce its length, so cuts it to be two hours. But then the director and actors protest, so she restores it to four hours. ]

The producer relengthened the film. (could be just one lengthening)

c. [ Kim takes a photo that is too large to use as a Facebook profile photo. She shrinks it to a more appropriate size, but thinks it does not look good. So she restores it to its original size and puts it on her personal website instead. ]

Kim reenlarged the photograph. (could be just one enlarging)

(51) a. [ A boutique store makes their shirts in the back. Sandy buys one and leaves with it, but then decides she does not want it. She goes back to the store with the shirt and exchanges it. ]

\#Sandy rereturned the shirt.

(necessarily two returnings)

b. [ Leah kills a rabbit, takes it home and skins and butchers it and then puts the fresh meat in the freezer for three days. She then takes it out and puts it on the table to thaw. ]

\#Leah rethawed the meat.

(necessarily two defrostings)

c. [ An ice cream factory manufactures ice cream from a package of ingredients by adding water and then freezing the result. After adding the contents of the package to water and freezing it, Kim lets it melt into a liquid state. ]

\#Kim remelted the ice cream. (necessarily two defrostings) 
d. [ John is hiking in a very hot region and comes across a fruit that has brown, fatty edges. He picks it and takes it back to his house where he trims off the edges and puts it in the fridge. He later takes it out and fries it. ]

\#John refried the fruit.

(necessarily two fryings)

Assuming a uniform syntactic decomposition for the two classes of verbs, as we have in this section, and furthermore that $r e$-is only low-attaching, as commonly assumed in the literature, these facts can only be made sense of if the roots of result verbs come with an entailment of change, by contrast with the roots of PC verbs. In this way, even on a low attachment the entailment of change is preserved since re- has scope over the full meaning of the root and result roots entail change. In short, syntactic decomposition can nicely account for the semantic contrasts, capturing facts about sublexical modification along the way, provided that Bifurcation is dispensed with and that we allow for a contrast in the lexical semantics of the two root classes.

A potential objection to this line of argumentation, however, comes from the reversative analysis of again in Deo et al. (2013), Pedersen (2014), and Beck and Gergel (2015). Roughly following the version of this analysis sketched in Deo et al. (2013: 111-2) again always modifies a verbal structure (i.e. never a root), and the semantics is that there is a reversal of a prior change. The restitutive reading is where the prior change is from being in a state described by the root to not being in such a state, while the repetitive reading is where the prior state was in turn the result of a change. On such an analysis, crucially, there is no scopal ambiguity, and in particular again never scopes over the root directly, meaning it is not technically a probe purely for root semantics divorced from the rest of the event structure. Nonetheless, it is not clear how this analysis would explain the absence of a restitutive reading with result root verbs-if the verbal structure modified by again entails the existence of some simple state divorced from a change then it should be possible to say that the one time change into that state is being reversed.

Assuming the worst, however, and supposing that the lack of root attachment somehow compromises the conclusions drawn from the again data or the redata, the point still holds once we shift to other classes of sublexical modifiers. In particular, Spathas (2017), in a study of Greek which also applies to English, shows that additive modifiers equivalent to English also and too show exactly the same point in a way that requires a scopal analysis. In particular, (52) (with focus intonation on the object) allows one reading where John performed the same action to another entity, and another reading where this is the first time John has performed this action but there is another entity that is in the described state, the former analogous to a repetitive reading and the latter a restitutive reading where the patient of the prior event/state is different: 
(52) a. John flattened the RUG too, so that it matched the drapes, which were sewed flat.

b. John flattened the RUG too, after having flattened the drapes.

As Spathas shows a reversative analysis will not work here, since there is no prior change to reverse. Instead, a scopal analysis is required. Crucially, as Spathas also shows, the PC vs. result root distinction found with again obtains with also, which he illustrates for Greek, but his English translations behave identically (Spathas 2017: 10, 16, (76) gives data for petheno 'die', skotono 'kill', kurazome 'get tired', filakizo 'imprison', skuriazio 'get rusty', vutirono 'butter', skonizome 'get dusty', etimazome 'get ready', stejnono 'dry', adjazo 'empty', isiono 'straighten', orimazo 'ripen' for PC roots and spazo 'break', ftiahno 'fix', liono 'melt', ragizo 'crack', anatinazo 'explode', vrisko 'find', eksafanizome 'disappear', pnigo 'drown' for result roots):

(53) a. [ Yesterday, John bought some new pants and a new shirt but dropped them near some water right after he got out of the store. The pants stayed dry, but the shirt got very wet. At home, when he put both in the washing machine...]

Stegnose ke to PUKAMISO.

dried also the shirt

'The SHIRT dried too'

b. [ Last week, Mary bought a new TV and a new laptop. Three days later the laptop was working fine, but the TV wasn't. Very upset, Mary brought her tools and ... ]

\#I Maria eftiakse ke tin TILEORASI.

the Mary fixed also the television

\#'Mary fixed the TELEVISION too.'

(based on Spathas 2017: 10-11, (43)-(44), (47)-(48))

Thus additional sublexical modifiers support the point that with result root verbs it is not possible to (semantically) assert just the prior existence of the state divorced from the change that led to it, even controlling for whether the relevant reading is derived scopally or not.

The approach to the morphological distinction between the two root classes is less obvious. Any analysis needs an explanation of the crosslinguistically robust fact that result roots are generally not found in basic state contexts, while PC roots typically are. As it stands, and as demonstrated in (40), the current analysis ensures that a PC root Merging directly with an adjectivalizing head gets the basic state meaning, while a result root gets a result state reading, correctly predicting that the two roots should differ in this way. The crosslinguistic work surveyed in $\$ 2.3 .2$, however, suggests that result roots rarely appear in these basic state contexts in the first place, raising the question of whether such structures should be generated at 
all, or if they are, how we can ensure that their morphophonological realization is systematically different to the realization of PC roots in the same contexts.

One way in which an analysis like the one sketched in this section could capture this contrast is with the assumption that purely stative meanings are unmarked as adjectives while roots with change in their meaning are marked as adjectives (in an iconic way reminiscent of Haspelmath 1993). If one strictly assumes that all lexical decomposition is syntactic, then this morphological contrast might be formally understood as root-conditioned allomorphy of adjectivalizing and verbalizing heads-null if the root has the appropriate unmarked reading and marked otherwise (though this could be overridden by root-specific rules to account for any item by item lexical idiosyncrasy): ${ }^{22}$

(54) Default realization for Asp with complement $\mathrm{X}(\operatorname{root} \sqrt{\mathrm{R}}$ or $v \mathrm{P})$ :

a. If $\mathrm{X}$ does not entail change, then $-\varnothing$

( $\mathrm{PC}$ roots derive unmarked adjectives)

b. If $\mathrm{X}$ entails change, then -en/ed

(result roots derive marked adjectives)

This yields a morphological component to the verbal typology discussed here that again arises from the root and not (solely) the templates the roots occur in. An alternative might have it that result roots lack spell outs when combined solely with adjectivalizing heads and thus must always combine with $v_{\text {become }}$ first, which in turn generates the appropriate morphology, making all adjectives with result roots literally deverbal. ${ }^{23}$ It does bear mentioning that analyses such as these are not consistent with some lines of thinking in DM which have it that Spell-Out and semantics are independent of one another (Marantz 1996). Alternatively, the spell out for each root could, of course, be lexically listed. But given the crosslinguistic generality of the semantic contrast the pattern simply cannot be a morphological accident, and thus reducing it to a list would give rise to a clearly inadequate analysis of the morphology.

\subsubsection{A purely lexicalist analysis}

The debate between non-lexicalist and lexicalist approaches to event structure is one we believe hinges on facts outside the realm of those considered here. Our simple observation in this book is that Bifurcation, independent of framework,

\footnotetext{
${ }^{22}$ See also the conceptually similar markedness conventions tied to decomposition and realization as adjective or verb in Lakoff (1965: Appendices A, C) and Green (1974: 22) for some discussion of them.

${ }^{23}$ See Pross (2019) for a related argument from the domain of German participial morphology. Something similar would need to be said about the morphological asymmetry in the change-of-state verbs themselves documented in Beavers et al. (2018). See that study for preliminary thoughts on the matter.
} 
must be abandoned. In this section we consider what a lexicalist analysis of the facts discussed in this chapter might look like. As discussed in \$1.3.1, where a lexicalist analysis breaks with its non-lexicalist alternatives is in having word formation take place before syntax, so that it is fully formed words entering the syntactic derivation, though these words are nonetheless associated with event structures built from basic event-denoting primitives and lexical semantic roots analogous to the functional heads and idiosyncratic morphological roots of a syntactic event structural analysis.

The most straightforward lexicalist analysis that would capture the contrasts discussed above is to assume distinct categorial and semantic lexicalizations tied to the two lexical semantic root classes, with lexical derivations based on this initial lexicalization (cf. Koontz-Garboden and Levin 2005, Koontz-Garboden 2005, 2006). ${ }^{24}$ Quite what these distinct lexicalizations will be will vary depending on the nature of the language analyzed as well as the nature of the root. Starting with result roots in English, the most obvious analysis is that lexical semantic roots that fall into this class are generally lexicalized as change-of-state verbs rather than as adjectives, and any categories derived from this unmarked lexicalization will thus be deverbal. Whether the lexicalization of the change-of-state verbs is basically as a causative or as an inchoative would not bear on the matters discussed here. As noted in $\$ 2.2 .1$ there is crosslinguistic variation in which of the two verbal forms is the more basic one, though there are some root meaningspecific tendencies that figure into what the basic form is even internal to single languages (Haspelmath 1993: 104). Result verbs like break, for example, are most commonly morphologically simple causatives in Haspelmath's study, with the inchoative derived by some anticausative derivation. Thus for the sake of argument we could assume lexicalized event structures for result roots as caused changeof-state verbs similar in spirit to what was proposed in Dowty (1979: 200-5) where they are logical formulae with decompositional structure that sublexical modifiers could be sensitive to. We could thus assign crack an event structure like that in (55), augmenting the Dowty-style representations with Davidsonian event variables (as e.g. in Rothstein 2004 and much other work) and assuming the lexical semantic root (treated here not as a morphological object, but we use the same $\sqrt{\text { Rоот notation for expository purposes) would have the same truth conditional }}$ interpretation as $\sqrt{\text { CRACK in }(37 b):{ }^{25}}$

\footnotetext{
${ }^{24}$ A reviewer asks what is meant by lexicalization in this context. By it, we mean the pairing of a root with a template, an interpretation, and phonological form, with the pairing (the lexeme) assigned a lexical category, as in the foundational work on event structures discussed in \$1.3.1, and ultimately going back to Dowty (1979).

${ }^{25}$ Although we suggest lexicalization as causative with the inchoative derived for result roots, we consider this ultimately an empirical question, and not one that matters for the purposes of contrasting the various flavors of analysis discussed in this section, since a lexicalist analysis could equally assume lexicalization an an inchoative with the causative derived. What is crucial is not the direction of derivation between causative and inchoative, but rather the fact that with result roots the verb is not derived from a basic adjective.
} 


$$
\llbracket \operatorname{crack}_{V} \rrbracket=\lambda x \lambda y \lambda v \lambda e \lambda s \exists P[[P(y, v)] \operatorname{CAUSE}[\operatorname{BECOME}(e, \sqrt{\operatorname{CRACK}}(x, s))]]
$$

If we assume that the truth conditions that define the operators such as CAUSE and BECOME are equivalent (with appropriate modifications) to those of the translations for the corresponding functional heads in syntactic event structures, semantically this analysis is equivalent to the syntactic one. And much in the way that we stipulated different types of change in the roots of crack verbs and cooking verbs above, we could do the same in the meanings of such verbs under this analysis in an identical fashion (e.g. the lexical semantic root of cook would impose a condition ensuring that $e$ be in $U_{E}$ ). Assuming a compositional analysis of anticausativization as in Chierchia (2004a), Koontz-Garboden (2009a), Beavers and Koontz-Garboden (2013a,b), and Beavers and Zubair (2013) inchoative variants of lexically causative verbs would be derived from the causative and thus would inherit the meaning of the latter, as does indeed seem to be the case for result verbs in many familiar languages with overt morphological marking of anticausativization. Morphologically, for result roots given the basic lexicalization as a verb with the denotation in (55), any stative form sharing the same root will have to be derived from the verb, as is transparently the case in languages like English.

By contrast, PC roots could be lexicalized as basic stative words (adjectives in English) with a denotation like that we have proposed for basic adjectives under the non-lexicalist analysis, having the truth conditions in (40b) because the root's meaning has the same truth conditions as (37a) and there is otherwise no additional templatic semantics, meaning no change is introduced anywhere:

$$
\llbracket \mathrm{flat}_{A} \rrbracket=\lambda x \lambda s[\sqrt{\operatorname{FLAT}}(x, s)]
$$

This makes several predictions. First, PC words are morphologically simple in their state-denoting form rather than morphologically complex (even if only covertly so) as in an analysis where such words are formed in the syntax from precategorial roots. Second, these words do not entail change since neither the template nor the root gives rise to such an entailment. Third, change-of-state verbs based on these roots will necessarily be morphologically complex since they must be derived from the morphologically basic and lexically-listed adjective, a prediction which Beavers et al. (2018) show to be correct across languages. ${ }^{26}$ Fourth, given a general

\footnotetext{
${ }^{26}$ The generalization is slightly more complicated than this, given that there are many languages in which the basic state property concept term is not an adjective but rather a noun or a verb. Nonetheless, the key observation is that the simplest form tends to be the basic state one when there is a clear markedness asymmetry between the stative and change-of-state members of the paradigm, whatever category the basic state term happens to be, with change-of-state senses usually derivationally related (with the exception that change-of-state senses are sometimes coerced from states rather than derived in languages in which property concept words are verbs; Koontz-Garboden 2007, Matthewson et al. 2015).
} 
process of deverbal adjective formation the existence of minimal pairs (e.g. red versus reddened) is predicted, in contrast to break roots. In particular, given the lexicalization of result roots as basic change-of-state verbs any adjectives formed from these will necessarily be deverbal, directly predicting the basic state gap. Finally, the derivational relationship between causative and inchoative for PC verbs is not predetermined by this analysis, and may differ to that of alternating verbs based on other states, as has previously been observed for at least some languages (Megerdoomian 2002, Alexiadou and Anagnostopoulou 2004, KoontzGarboden 2006).

The core idea of this analysis, then, is that there is a contrast in lexicalizationbasic state denoting adjectives in the case of PC roots vs. change-of-state-denoting verbs in the case of result roots-rather than in special Spell-Out rules. This derives the distinct morphological properties of the two paradigms of words rooted in the two different root classes as artifacts of the basic category plus subsequent derivation, though on both the lexicalist and syntactic analyses there is a core shared intuition that some kind of form-to-meaning markedness correlation obtains (in what is the unmarked lexical category for each root type on the lexicalist analysis vs. what root triggers zero-realized categorial heads in the syntactic analysis).

However, a set of facts that may distinguish between these two analyses are those involving sublexical modification. A purely lexicalist analysis along the lines articulated above is not without some complication with regard to restitutive modification in particular, which, as articulated by Dowty (1979: 264-9), requires positing an ambiguity in again between one use that triggers a repetitive reading fairly straightforwardly (by modifying the verb or verb phrase itself) and one that modifies the verb but ends up scoping over just the root by virtue of some meaning postulate operating over lexicalized event structures. A theory like this can capture the facts, including the contrast in availability of restitutive modification, given that the restitutive again would still on this analysis scope over a lexical semantic root whose denotation includes change in the case of result roots but lacks change in the case of PC roots. ${ }^{27}$

Nonetheless, such a treatment is deemed ad hoc by some (see e.g. von Stechow 1996: 130-2), though to some degree this is a matter of theoretical taste given that a syntactic decompositional analysis of verb meaning has properties of its own that

\footnotetext{
${ }^{27}$ This analysis does assume that the meaning of again is not as in Jager and Blutner (2003: 404, (26)), where restitutive again applies to the verb and through some purely truth-conditionally defined semantic operator somehow pulls out a result state. We assume its meaning is instead dependent on the event structure, so that the entire meaning of the root, whatever it may be, is under the scope of again. This is tied to the fact that a purely lexicalist analysis does not obviate the need for a root/template distinction and event structure more generally, which is required not just for accurate characterization of sublexical modification facts discussed immediately above, but other facts discussed in $\$ 1.4 .3$, e.g. that roots act as scopal units and that templates determine various asymmetric prominence facts about arguments whether it is conceived of lexically or as part of the syntax. See also $\$ 2.6, \$ 3.10$, and $\$ 4.7$ for further discussion.
} 
are arguably ad hoc (e.g. the need to posit functional heads for which there may be little to no independent evidence; see also $\$ 1.3 .1$, fn. 4). Another problem raised by von Stechow (1996: 131) is that such an analysis, at least as articulated by Dowty (1979), does not capture the syntactic restrictions on the restitutive/repetitive readings of again (or its German counterpart) regarding position of the again modifier, where preverbal or presentential again lacks a restitutive reading altogether. Further, Bale (2007: 461-3) points out that additional intersective adverbial modification on the restored state makes the meaning postulate analysis difficult because it precludes access to the event structure in the way proposed by Dowty. Our goal here is not to solve these kinds of problems, but rather to highlight the merits and problems with different kinds of analysis consistent with the facts we have discussed in this chapter. This particular problem is not a problem with an analysis that gets rid of Bifurcation, but rather a problem in dealing with the facts of restitutive modification in a lexicalist analysis more generally.

\subsubsection{A mixed analysis}

Another style of analysis would mix aspects of the lexicalist and non-lexicalist analyses above, with word formation largely syntactic but the syntactic terminals always categorial. Thus while derivational processes find their locus in the syntax, the actual categorization of words does not (see \$1.3.2). ${ }^{28}$ Although there are many ways of proceeding with these assumptions, on such a theory, PC roots would be lexicalized as adjectives and result roots as verbs, just as in the lexicalist analysis, but all subsequent word formation is syntactic, e.g. the derivation of PC adjectives into verbs happens in the syntax via functional heads and the result root verbs may (for example) be lexicalized as inchoative verbs and then derived into causatives syntactically as per Kratzer (1996).

For both kinds of roots, deverbal adjectives would be derived syntactically from the verbs. On such an approach, the variation across roots in terms of their morphological properties regarding the causative alternation-whether the inchoative or the causative is the unmarked member of the pair-might be treated as in Doron (2003), where the event structures are the same for all causatives and for all inchoatives but the spell out of the relevant functional heads differs depending on the root class. ${ }^{29}$ This analysis comes automatically

\footnotetext{
${ }^{28}$ Something like this seems possibly what Baker (2003: 269, fn. 2) has in mind, and might be a way of reading Hale and Keyser (2002).

29 This approach is not without problems, however, since it does not capture the causative entailments that even inchoatives seem to have in some languages noted in \$2.2.1; see Koontz-Garboden (2009a), Beavers and Koontz-Garboden (2013a,b), and Lundquist et al. (2016) for further discussion. Also, the above is not to say that root class alone might be the sole determining factor of the morphophonological shape of the verb in Hebrew.
} 
with a straightforward analysis of restitutive modification: restitutive vs. repetitive modification again arises as a syntactic attachment ambiguity, where restitutive scope is over the underlying adjective of PC verbs and the inchoative core of result verbs - the smallest attachment point in each case-the former of which does not also have entailments of change but the latter does. Such a theory would straightforwardly capture the morphological contrasts by, much as in the lexicalist analysis, setting up two grammatical categories of root. Also, like the non-lexicalist analysis, it provides a straightforward explanation of the facts tied to restitutive modification.

\subsubsection{Summary}

The three analyses sketched here-one accepting purely syntactic categorization and word formation, one placing all categorization and word formation in the lexicon, and one mixing those two approaches-share in common a single key assumption that handles the basic facts, namely that the roots of PC and result verbs differ systematically in that the latter lexically entail change and the former do not. Ultimately, independent of the theoretical particulars, it is this analytical contrast which we believe the facts discussed in this chapter point toward, but which is fundamentally inconsistent with Bifurcation. The specific details of each theory (which were just sketched above) represent the kinds of trade-offs different sets of assumptions make in capturing the morphological and sublexical modification facts, though in each case something-a theory of lexicalization of word categories based on the type of the (lexical semantic) root or a theory of SpellOut of categorizing morphology-must be assumed to capture the basic correlation that (lexical semantic) roots lacking entailments of change form unmarked adjectives and those bearing them form unmarked verbs.

Cutting across the implementational details, though, the sublexical modification facts always hinge on having a single lowest scopal point entailing both a state and a change simultaneously. Furthermore, the morphological markedness correlations should ultimately not be too surprising: since change is itself already a semantic category that has grammatical consequences-being for example the basis of $v_{\text {become }}$ and its equivalents in other theories-then if processes in a language are already sensitive to this type of meaning it is unsurprising that they might treat roots entailing this meaning differently from roots that do not, however this is implemented. In sum, the root carries an entailment that can be elsewhere introduced templatically, and it can matter grammatically. But this is all, as noted, inconsistent with Bifurcation. For this reason, we next consider analyses that do not reject Bifurcation to see how (and how well) they account for the same sets of facts. 


\subsection{Analytical option 2: Preserving Bifurcation}

While we believe that the facts discussed in this chapter argue strongly against Bifurcation, maintaining it is still technically possible. In this section we critically outline some of the ways in which a theory could still maintain it while capturing the facts discussed in this chapter, at least to some degree. The key fact is that in all of them some other distinction between PC and result roots must be posited to explain their distinct behavior other than the meanings of the roots. However, as we show the different types of distinctions and the theories that derive the relevant facts from those distinctions are often lacking either empirically or theoretically.

\subsubsection{Reanalyzing result roots as manner entailing}

Embick (2009) considers an analysis of the facts discussed above that would preserve Bifurcation, assuming result roots have a fundamentally different syntactic and semantic status than they do on a more traditional analysis. In particular, rather than being state-denoting and having the syntax expected of a statedenoting root as in (57a) for PC roots, result roots have a manner meaning and merge in the position of manner modifiers, i.e. as adjuncts of some $v$ as in (57b) (Embick 2009: 6, 17). (In both cases Embick has the patient as a complement to the stative head rather than as a specifier of a $v$ as we have had it above; this distinction is irrelevant for present purposes.)

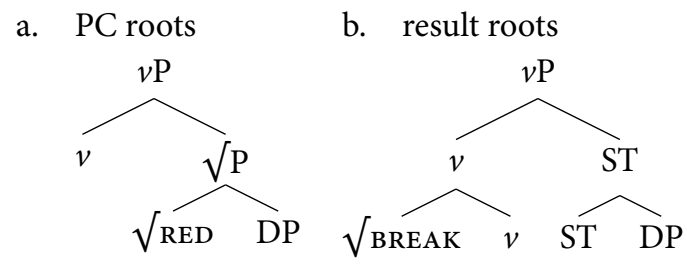

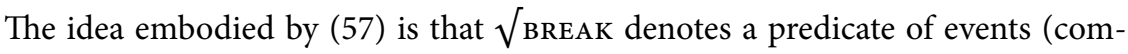
posing with $v$ by predicate modification) and takes an unspecified state (ST) as an argument. In this way, it has a denotation on a par with the roots of manner verbs like pound which imposes entailments on the agent of a pounding, but says nothing about the resulting state of such an event.

Embick's proposal is made absent a theory of what exactly manner is semantically. However, in $\$ 4.3$ we review diagnostics for manner based on Rappaport Hovav (2008) and Rappaport Hovav and Levin's (2010) idea that manner is nonscalar. Part of this will involve showing that break in particular-and also other so-called canonical "result verbs" that are based on result roots-entail a result but crucially no manner. For this reason we defer a full accounting of the fact that 
break and its ilk do not entail a manner until then. Instead we just presage that discussion by making a couple of observations.

First, verbs that impose manner-oriented lexical entailments on one of their arguments lexically specify the nature of an action of some sort, generally making it possible for only certain types of entities to perform the actions described by the verb, thus restricting the possible subject DPs the verb can take (see \$1.6.2.1). Thus canonical manner verbs like scrub or wipe only permit subjects that can be said to be able to perform scrubbing or wiping actions respectively, precluding inanimates (except when interpreted as instruments manipulated by an unexpressed agent; see $\S 1.1$, fn. 2) and natural forces:

(58) a. John scrubbed/wiped the floor with a stiff brush.

b. ??The stiff brush scrubbed/wiped the floor.

c. ??The earthquake scrubbed/wiped the floor.

d. ??The pressure from the water scrubbed/wiped the submerged floor.

Verbs like break or shatter on the other hand are well-known to allow a range of subjects (see e.g. Levin and Rappaport Hovav 1995: 85; 102-6), something that is not incompatible with entailing a manner of some sort for the subject but certainly does not offer any support for that conclusion: ${ }^{30}$

(59) a. John broke/shattered the vase with a hammer.

b. The hammer broke/shattered the vase.

c. The earthquake broke/shattered the vase.

d. The pressure from the water broke/shattered the windows of the submerged car.

${ }^{30}$ One might conclude that the fact that lack of thematic restriction on the subject of a transitive change-of-state verb strongly correlates with participation in the causative alternation (Levin and Rappaport Hovav 1995: 85 and Reinhart 1996: 17 and also \$2.2.1) that this alternation is actually a diagnostic of a lack of manner entailments on the subject of the causative. However, such a conclusion is too strong, for two reasons. First, it presupposes an underlying analysis of the causative alternation from which it actually follows that absence of manner should correlate with alternating. Although this is arguably the case for the reflexivization analysis (Chierchia 2004b, Koontz-Garboden 2009a), it is likely not for other analyses (see Koontz-Garboden 2009a: 123-34 for discussion), and the reflexivization analysis remains controversial (see Koontz-Garboden 2009a, Horvath and Siloni 2011, 2013, Beavers and Koontz-Garboden 2013a,b and Lundquist et al. 2016 for extensive back and forth on the issue). Second, more generally, there is reason to tread carefully because use of the alternation as diagnostic of manner presupposes a more thorough understanding of what manner is than is currently available. As we discuss in $\$ 4.3$, although it is possible to identify particular classes of entailments that cannot be understood as anything other than manner, the state of the art is not yet such that it is possible to understand manner generally. The possibility therefore remains that even those verbs with thematically underspecified external arguments could have manner entailments imposed on them for which we do not yet have tools to diagnose. As discussed in $\$ 4.3$, it is preferable to use positive diagnostics for particular kinds of manners, rather than negative diagnostics for lack of manner altogether, which is the kind of diagnostic that the causative alternation would be. 
This goes hand in hand with the fact that with human subjects, which are acceptable with both a canonical manner-entailing verb like scrub and also with a verb like break, it is difficult to deny that the subject performed any action at all in the former case but easier in the latter case:

(60) a. \#Jim scrubbed/wiped the table, but he didn't move a muscle.

b. Kim broke/shattered my DVD player, but she didn't move a muscleinstead, despite being in charge of taking care of it, she just negligently let the rotor run until it spun out of control and damaged the whole thing.

Now, it could be that the manners associated with break or shatter are not of the sort that involve physical motion of the body per se despite the fact that these two verbs describe changes-of-state that are real world, physical changes. If there is manner there, however, it is unclear what it would be in the case of these two verbs. Absent a more specific claim, the more likely assumption is that there is no manner in the meanings of these two verbs at all, consistent with the orthodox view on the meanings of such verbs. In sum, we do not believe that there is any evidence for the claim that result roots are manner entailing. (See Chapter 4 for further discussion.)

\subsubsection{Allosemy}

The fundamental problem faced by any analysis that preserves Bifurcation is that the roots of result verbs fail to generate the expected inferences in the expected contexts if they were indeed purely stative. This, in turn, seems to be reflected in the morphology. But leaving that problem aside, another key question is how to capture the fact that the roots of result verbs give rise to an inference of state change in contexts where they are not expected to. There are two principal contexts of concern: basic state constructions and restitutive modification as with again and re-. In this section we consider an allosemic analysis of parts of the structure of change-of-state verbs and adjectival derivatives in the spirit of Myler (2014) and Wood and Marantz (2015) which would correctly capture the facts. We argue, however, that such an analysis does so at a theoretical cost in that it requires positing denotations for functional heads that appear in just the places they are needed to make the analysis work and nowhere else and which are not independently motivated by any facts outside this particular domain.

We consider first the adjectival structures. As discussed in $\$ 2.2 .1$ a core problem for Bifurcation is that just as PC roots appear in basic adjective structures like (61a) we also expect result roots to as well as in (61b): 
(61) a.

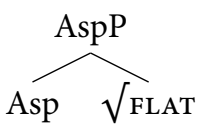

b.

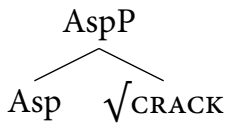

The question then is how to ensure that by contrast with (61a), (61b) gives rise to the (templatic) entailment of change that cracked and its result-root ilk are known to give rise to without locating that entailment in the root itself. A possible answer comes from an allosemy analysis whereby functional heads can vary in their meaning depending on the syntactic context in which they appear. Suppose, then, that there are actually two different interpretations for Asp, as in (62):
a. $\llbracket$ Asp $\rrbracket=\lambda P[P]$
b. $\llbracket$ Asp $\rrbracket=\lambda P \lambda x \lambda s\left[P(x, s) \wedge \exists e^{\prime}\left[\right.\right.$ become $\left.\left.\left(s, e^{\prime}\right)\right]\right]$

If we assume root meanings for both $\sqrt{\text { FLAT }}$ and $\sqrt{\text { CRACK }}$ as in (63) with no templatic meaning (i.e. cracked' is interpreted with no lexical entailment of change), consistent with Bifurcation, the allosemic idea would be that in (61a) Asp is interpreted as in (62a) while in (61b) it is interpreted as in (62b):
a. $\llbracket \sqrt{\mathrm{FLAT}} \rrbracket=\lambda x \lambda s\left[\mathrm{flat}^{\prime}(x, s)\right]$
b. $\llbracket \sqrt{\text { CRACK } \rrbracket}=\lambda x \lambda s\left[\operatorname{cracked}^{\prime}(x, s)\right]$

The driving force behind the difference in interpretation would be the specific root Asp Merges with-it is interpreted one way with some roots and another way with others, so that in particular in (63a) we only ever get Asp interpreted as (62a) while with (63b) we only ever get Asp interpreted as (62b). Compositionally, this leads to different interpretations for the adjectival structures rooted in flat and crack respectively as in (64), with a basic state interpretation for the former and a result state interpretation for the latter:
a. $\llbracket[\operatorname{Asp} \sqrt{\text { FLAT }}] \rrbracket=\lambda x \lambda s\left[\right.$ flat $\left.^{\prime}(x, s)\right]$
b. $\llbracket[$ Asp $\sqrt{\text { CRACK }] \rrbracket}]=\lambda x \lambda s\left[\operatorname{cracked}^{\prime}(x, s) \wedge \exists e^{\prime}\left[\right.\right.$ become $\left.\left.^{\prime}\left(s, e^{\prime}\right)\right]\right]$

The situation will be similar for $v_{\text {become }}$ on an analysis of this sort; as discussed above in relation to the typology (30), different roots select for different varieties of change, with some, like the cooking roots, only allowing for temporal change, and others, like break roots, allowing for both temporal and atemporal change. Given 
that pinning this difference on the relevant roots is not a possibility if one assumes Bifurcation, there will have to be at least a temporal alloseme of $v_{\text {become }}$ alongside either an atemporal or an underspecified one. A similar additional polysemy for Asp will also be required, since the same roots presumably only allow temporal change in adjectival forms as well. Which alloseme appears with which root will then be determined root-specifically, in a manner much like how the interpretation of Asp in (64) depends on which root it appears with.

While this analysis gets the basic semantic facts right, it still does not immediately capture the restitutive modification facts with again and re-, since these are assumed to attach to the root on restitutive readings and the root in both cases will be a basic state, thus failing to generate the contrasting readings with $\mathrm{PC}$ roots versus result roots. A simple fix would be to posit that, contrary to assumptions in the decompositional literature as embodied in structures for change-of-state verbs like those in (31c) and (31d), repeated in (65a,b), where inchoatives and causatives are built directly from roots, inchoatives and causatives are instead genuinely deadjectival, so that $v_{\text {become }}$ takes as its complement an AspP, as in (66a,b):

(65) a. Inchoatives under the standard theory (Embick 2004: 365)

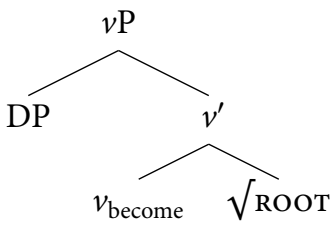

b. Causatives under the standard theory (Embick 2004: 366)

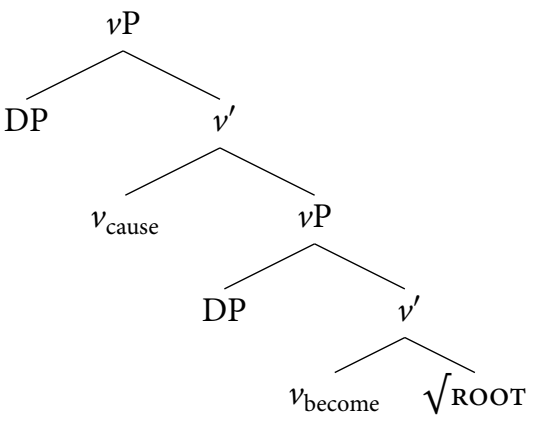

(66) a. Inchoatives under the deadjectival theory

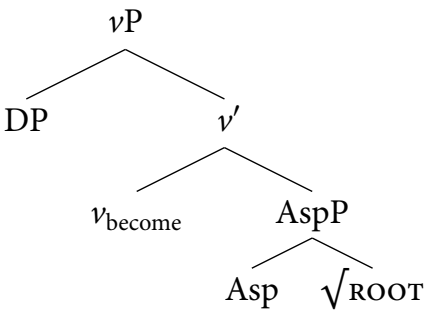


b. Causatives under the deadjectival theory

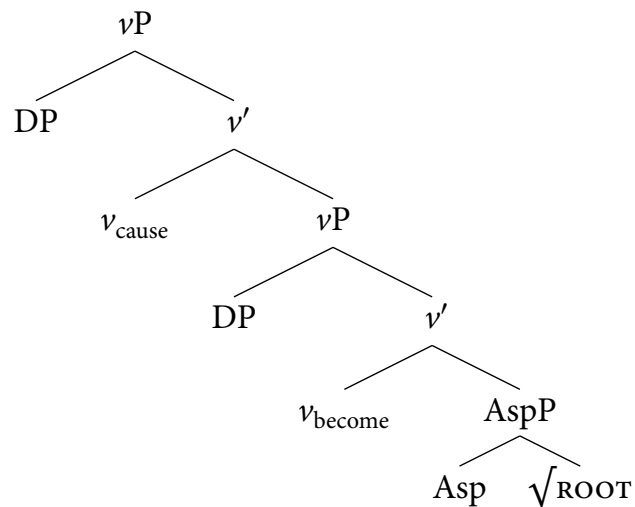

If we furthermore assume that again cannot scope directly over roots but instead over AspPs, then the contrast in restitutive modification can be captured, since Asp in the context of a PC root is interpreted as (62a) while in the context of a result root it is interpreted as $(62 \mathrm{~b})$, thereby ensuring that no real restitutive reading is available with result roots even under low attachment.

While this constellation of assumptions does save Bifurcation, it raises some questions. First, it relies on novel and as yet unmotivated theories of (i) the syntax of change-of-state verb formation and (ii) the syntax of restitutive modification (again, given the received view discussed above that restitutive re-attaches to the root). Second, it remains unclear on this theory why, as discussed in $\$ 2.3 .2$, PC roots have basic state forms (basic adjectives in English) that result roots overwhelmingly lack across languages. If result roots really do appear in the same basic state structure as PC roots, why do we not see more morphosyntactic evidence of it? Finally, a more high-level consideration is the question of where precisely the right place is to put idiosyncrasy in the grammar. The allosemic theory ultimately puts it in the syntax, making the difference in meaning between the two classes of adjectives a consequence of the combination of elements in context. It may be a matter of theoretical taste where such idiosyncrasy is best placed. Our intuition is that building this contrast into the meanings of the roots themselves gives rise to a theory with a range of additional desirable properties. This is largely because, as we have suggested briefly in $\$ 2.4 .1$ and discuss more in $\$ 5.2$, the entailment of change might be best thought of as arising from the nature of the state itself and presumably the same kinds of states have the same kinds of properties across languages. In other words, the intuition we outlined above is that result roots are those that describe states that are conceived of independently as arising from some change, while PC roots describe those that do not require this. Thus the entailment of change is a part of the meaning of the former and not the latter owing to non-linguistic conceptual properties of different sorts of states. 
Yet this is not a property of the roots on the allosemic theory. Rather, it is an idiosyncratic property of the functional head in combination with particular roots, which must be listed on this theory. By assuming Bifurcation, roots cannot differ in meaning in the appropriate way, raising the question of why the same kinds of states across languages generally only appear with one interpretation of the functional head, while other states only appear with the other. If it is assumed to actually be based on the meanings of the roots, then the question becomes why we would need allosemy anyway since the root already has the relevant component of meaning built into it and the facts would thus follow without the allosemy theory. So the allosemic analysis has stipulations in it that, once resolved, obviate the need for allosemy. There may, of course, be other reasons for why a theory of allosemy in the context of DM is necessary (as discussed by Myler 2014 and Wood and Marantz 2015). We remain agnostic on that question and are simply suggesting that saving Bifurcation by appealing to such a theory is unwarranted.

\subsubsection{Root selection for syntactic construction}

Another conceivable analysis that would maintain Bifurcation, in the spirit of Alexiadou et al. (2006: 202, (51)) and Ramchand (2008: 12-5, 57-62), would be one in which roots can select for the functional context in which they appear through some kind of featural mechanism (or by some classification defined by intuitionistic semantic groupings of roots that are not technically truth conditional in nature). For example, we might say that all result roots come with a feature, call it $\left[+v_{\text {become }}\right]$, that requires them to Merge with a $v_{\text {become }}$ head. In this way, result roots would be prevented from appearing in the basic state context, since immediate Merge with Asp would fail to satisfy the $\left[+v_{\text {become }}\right]$ feature on the result root. PC roots, by contrast, would lack such a feature, so could Merge freely with Asp or $v_{\text {become }}$. Most likely a theory such as this would need to also posit atemporal and temporal flavors of $v_{\text {become }}$, alongside the associated selectional features, in order to capture the contrast noted above in (30) in what kinds of change result roots select for. ${ }^{31}$

As with the allosemic analysis, if we adhere strictly to Bifurcation so that change-of-state verbs built on result roots have their entailments of change (of whatever kind) introduced only by $v_{\text {become }}$ and not by the root at all, then the question arises of how to account for the observed contrasts in restitutive modification.

31 As noted in $\$ 1.3 .3$ this is similar in spirit to the analysis of Rappaport Hovav and Levin (1998: 108-9), wherein the lexicalized event structure associated with any lexical semantic root is determined by default associations of roots with particular event structures via canonical realization rules. On this analysis roots of break verbs for example are associated in the lexicon with caused change-of-state event structures. 
Here, we would have to assume that with result root change-of-state verbs the syntax of again and re- attachment is different to its syntax with change-of-state verbs built on PC roots, with again and $r e$ - unable to attach below $v_{\text {become }}$ with the former class of roots while they can with the latter. Such an analysis would then predict that there is no genuine restitutive reading with result verbs while there is with PC verbs. While researchers like von Stechow (1996: 109) and Beck and Johnson (2004: 120) have indeed argued that again (and its ilk) can have different syntax with different verbs, we are unclear what empirical arguments there might be to independently motivate this kind of contrast with again and re-, at least beyond the particular facts with again and re-that such an analysis is meant to explain.

More broadly, although such a theory offers the potential for capturing the contrast between result roots and PC roots in a way that maintains Bifurcation, it has drawbacks. First, it puts much of the idiosyncrasy in the syntax, and second, it relies on root-specific syntactic stipulations which mimic the lexical semantics rather than directly reflecting it. Again, given the crosslinguistic generality of the root class distinction, this kind of contrast will have to be replicated in the syntax of language after language-arguably the wrong place for such stipulation. However, if the locus of the stipulation is the actual semantics of different roots rooted in the kinds of states they describe, as we have suggested here and discuss further in $\$ 5.2$, then it raises the question of why we would want the featural specifications anyway since the semantics already do the job without need to replicate it in the syntax (see Beavers and Zubair 2013: 39 for a related discussion of the redundancy of replicating an independently justified semantic analysis of agentivity in terms of syntactic features).

\subsubsection{Summary}

In this section we have considered various ways to preserve Bifurcation while still capturing the data discussed here. While such approaches are certainly possible, we believe that they come with certain complexities that approaches that reject Bifurcation do not. This includes either a wholesale rethinking of the classification of result roots as manner roots which lacks little independent justification, or essentially placing the bulk of the explanation on functional heads or featural systems that are contingent on formal properties of the relevant roots without positing any semantic distinctions between the roots, despite the fact that the distinctions are more plausibly based on root semantics itself, thus obviating the need for such additional theoretical complexities. The question would be what, other than Bifurcation, would motivate such analyses. Until such justification is given, analyses that reject Bifurcation are preferable. 


\subsection{Roots vs. templates in change-of-state verbs}

At this point we return to an issue first raised in $\$ 1.4 .3$ : if roots can have the meaning of $v_{\text {become }}$ in them, is there any reason to posit that there is $v_{\text {become }}$ ? What value is there to maintaining the root vs. template distinction? Here we suggest that despite our conclusion about root meanings, there are clear reasons to continue to maintain this that echo the same issues we discussed in $\$ 1.4$.3. First and foremost, of course, is that some stative roots do not entail change, yet take that reading on in certain contexts (e.g. as with descriptively deadjectival inchoative and causative verbs). Here something must be contributing the meaning, and the obvious candidate is the syntactic environment, i.e. a templatic head like $v_{\text {become }}$. Second, we had noted in $\$ 1.4 .3$ that roots act as complete scopal units such that for any state that one entails no matter how complex it is in terms of the number of truth conditionally distinct lexical entailments in it, they will all always be under the scope of sublexical modifiers like again. This fact is reinforced and in fact demonstrated more dramatically with result roots in $\$ 2.4$. With result roots not just the entire state but also the prior change of state itself is under the scope of again on a restitutive reading, but crucially with PC roots the state and the change can be separated. Thus even though a root can introduce a meaning that can also be introduced by a templatic head, only when they are introduced by templates can they be separated. Roots themselves do not provide the kind of structure to the semantic representation that allow for templatic meanings to be separated from the more idiosyncratic portion of the root meaning. This thus reinforces the key point in $\$ 2.4 .1$ that a root vs. template distinction is justified and motivated on more morphosyntactic grounds, if not necessarily on purely semantic grounds as Bifurcation might have.

\subsection{Concluding remarks}

The Bifurcation Thesis predicts a range of homogeneous morphological and semantic behavior in the domain of change-of-state verbs, which we have shown not to be borne out by the facts. There turn out to be at least two distinct classes of roots of change-of-state verb, so far as morphological and semantic behavior are concerned. Result roots introduce not only entailments about the nature of the state, but also an entailment of change itself. Such roots contrast with PC roots, which do lack change entailments (consistent with Bifurcation). This semantic contrast is reflected in the morphology, where we find that the former type of root is not attested in the same morphological forms as the latter, and the failure to find these roots in the same morphological environments is crosslinguistically systematic; it is not simply an accident of English, as has previously been claimed. 
This classification of roots (incorporating also subclasses of result roots in terms of whether temporal change is required or not) produces a predictive verb class typology that derives solely from the roots of the relevant event structures and not (solely) from the templates themselves. We return to the question of root-based verbal typologies and their significance in theories of event structures in $\$ 5.4$.

We then considered the theoretical consequences of these observations, exploring in a variety of theoretical backdrops what it would take to preserve Bifurcation in the face of the empirical evidence. Fundamentally, maintaining Bifurcation requires a considerable number of theoretical assumptions to get the facts right, suggesting that the facts point more clearly in the direction of falsifying Bifurcation, with result roots having an otherwise templatic entailment of change as part of their core. The question, then, is how to incorporate this observation into a theory of argument structure and the syntax/semantics interface. We explored a variety of options, all with their various advantages and disadvantages and all requiring at least some stipulations of their own to fully capture the data, though fewer than analyses that do not reject Bifurcation. Ultimately, our point here is not to decide among these, since we believe it is in part a matter of theoretical taste and independent assumptions whose justification lies beyond the scope of this book. What is clear, however, is that in whatever way the facts are to be accounted for, they falsify Bifurcation.

The deeper question here is why this would be the case, and as we have suggested briefly in $\$ 1.4 .3$ and $\$ 2.4 .1$ we believe it has to do with how we conceive of different kinds of states and whether a state can exist without a change or whether a change is part of the very definition of a given state. Before we return to this in $₫ 5.2$, we turn to our next case study of templatic meaning being found in roots, namely templatic meaning found in the roots of ditransitive verbs of caused possession. 



\section{3 \\ The roots of ditransitive verbs of caused possession}

\subsection{Roots and templates in ditransitive verbs}

In the previous chapter we considered Bifurcation as applied to change-of-state verbs, the empirical domain for which one of the clearest versions of the hypothesis was first articulated and for which Bifurcation certainly seems plausible on the surface. However, as our investigation demonstrated, Bifurcation does not hold for the roots of change-of-state verbs. In this chapter we now consider an argument against Bifurcation by which we extend it to a novel yet much more challenging domain, namely ditransitive verbs of caused possession (Green 1974; Gropen et al. 1989; Pinker 1989; Goldberg 1995; Beck and Johnson 2004; Krifka 2004; Rappaport Hovav and Levin 2008; Beavers 2010, 2011a; Bruening 2010a,b; Harley and Jung 2015, inter alia). ${ }^{1}$

Descriptively speaking, ditransitives are three-argument verbs that take a causer subject, a theme direct object, and a third argument representing a goal or recipient of the theme. This third argument can be realized in one of two ways, either as an indirect object in a double object construction as in (1a) or else as an oblique marked by to as in (1b), the so-called "dative alternation."
(1) a. Mary sent John a letter.
b. Mary sent a letter to John.

These two constructions are in turn canonically associated with two distinct (albeit related) interpretations. The indirect object construction entails some notion of possession of the theme on the part of the indirect object, whereas in the to construction possession is not a requirement. This is evidenced primarily by the fact that only animates or objects otherwise capable of possessing can be indirect objects, whereas the to oblique can be a DP representing inanimates and things

\footnotetext{
1 This chapter represents a significantly revised version of Beavers and Koontz-Garboden (2017b), itself based in part on the earlier study in Beavers (2011a) arguing for an analysis of ditransitive verbs as verbs of scalar change.
} 
that cannot possess the theme. For example, a location such as London can be realized as a to $\mathrm{PP}$, but not as an indirect object, except in a context where London is interpreted as an entity that can possess (e.g. as "The London Office" or "The London Representative" or "Scotland Yard"; see Green 1974: 103-4 and also Oehrle 1976: 81):

(2) a. \#Mary sent London a letter.

b. Mary sent a letter to London.

This suggests that the two syntactic constructions are associated with separate types of event structures-one describing caused possession and the other notidentifiable syntactically by the expression of the recipient/goal.

A commonly cited implementation of this approach is that proposed by Harley (2003), who suggests that the two constructions involve a causal head (e.g. $v_{\text {cause }}$ ) that takes one of two dyadic PP complements: either one headed by $\mathrm{P}_{\text {have }}$ that takes two DP arguments and entails a possession relation between its specifier (the possessee indirect object) and its complement (the possessum direct object) or a PP headed by $\mathrm{P}_{\text {loc }}$ taking a theme specifier DP and a locational complement PP headed by to and entailing a co-location relation between them (though as we argue below these are not the ultimate semantics of the either construction; see also Rappaport Hovav and Levin 2008 and Beavers 2011a). These are represented in (3a) and (3b) respectively. Harley (2003: 32, (3)) does not give an explicit representation of how the verbal root combines with the structure, but we follow Harley and Jung (2015: 715-6) and assume the root indicates the manner of causation (as per others; see e.g. Goldberg 1995: 50-51) and thus is adjoined to $v_{\text {cause }}$ :

(3) a.

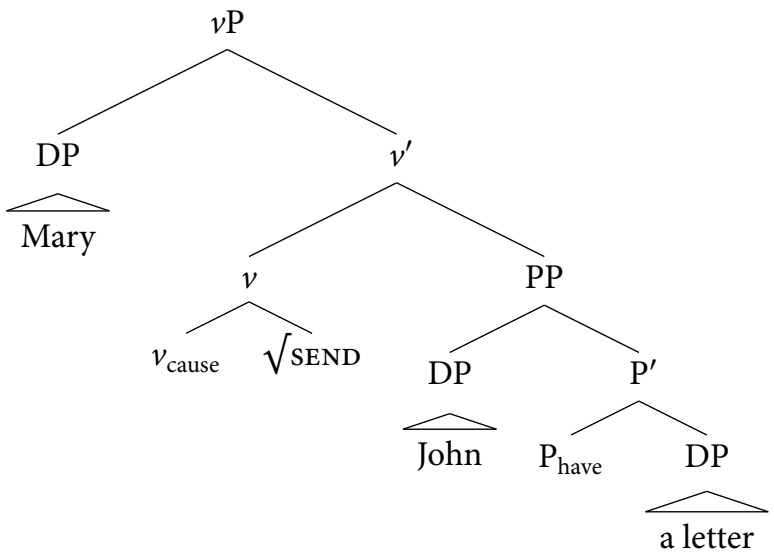




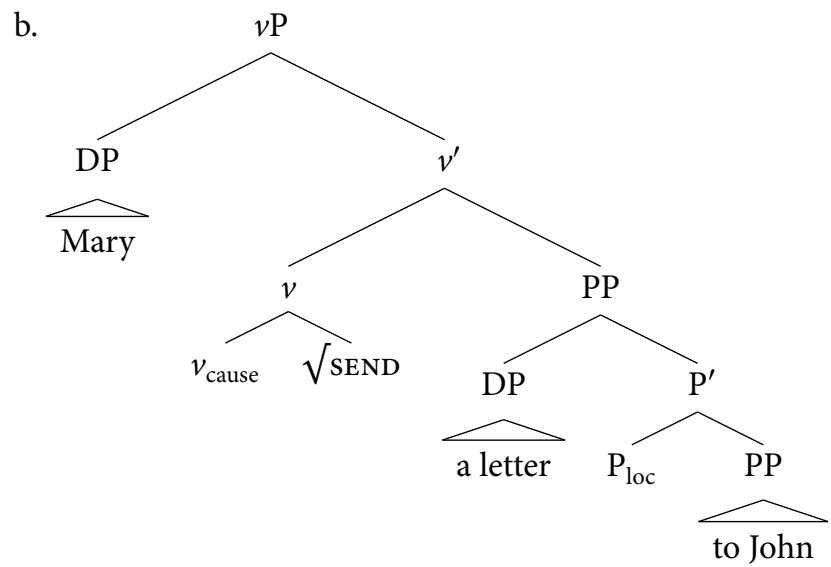

This analysis not only captures the correlation of the apparent semantic distinctions between the two variants illustrated above $-\mathrm{P}_{\text {have }}$ selects a possessor and thus generates a London Office effect while $\mathrm{P}_{\text {loc }}$ does not $^{2}$-and the syntactic realization, but also other facts about the alternation.

For example, it is well-known since at least Barss and Lasnik (1986) that whichever of the two complements is linearly first in the construction is capable of binding into the second but not conversely. Thus a quantifier in the first complement can bind a bound variable in the second but not conversely:

(4) a. Kim sent [ every employee $]_{i}\left[\right.$ her $_{i}$ paycheck ].

b. ${ }^{*}$ Kim sent $\left[\text { its }_{i} \text { employee ] [ every paycheck }\right]_{i}$.

(5) a. Kim sent $[\text { every paycheck }]_{i}$ to $\left[\right.$ its $_{i}$ employee $]$.

b. ${ }^{*}$ Kim sent $\left[\text { her }_{i} \text { paycheck ] to [ every employee }\right]_{i}$.

In an influential analysis, Larson (1988) analyzes these asymmetries in terms of different asymmetric c-command relations in the two different constructions defined by VP-shell structures, a structural aspect of the understanding of ditransitives that (3) preserves. These plus other facts suggest the overall plausibility of this analysis (though the analysis is not without problems; see Jackendoff 1990a; Larson 1990). We note at the outset that the particular analysis in (3) is not the only such analysis that has been proposed in the literature. For example, Pesetsky (1995: 123-71), Beck and Johnson (2004), Pylkkänen (2008: 12-9), Ramchand (2008: 100-6), Bruening (2010a,b), and Harley and Jung (2015: 715-6), inter alia,

${ }^{2}$ Evidence that this is the right analysis for why London is odd as an indirect object comes from the additional, more basic fact that \#London has a letter is also odd except on a "London Office" reading. The simplest explanation is that it has to do with a shared possessional semantics qua both being based on the same templatic head. 
all propose various syntactic decompositions that differ in the specific functional heads involved and their overall syntactic arrangement, but share the same basic broad semantic and structural properties of the analysis in (3). ${ }^{3}$ We adopt Harley's (2003) analysis here (with some modifications to the choice of heads, as introduced below) simply because it has been well cited and very influential. Nothing that we say below hinges on the specific details of the event structure provided that it captures the same basic syntactic and semantic facts.

Given this, the question arises what predictions Bifurcation makes regarding the semantics of the root. Since the root in this case supposedly entails the manner by which the causer causes the result state in each variant, then the prediction is distinct from but entirely analogous to the prediction made for the change-of-state verbs discussed in the previous chapter. In particular, the root should describe pure actions of some kind (as opposed to pure states as with change-of-state verbs), but nothing templatic. In other words, the root should not entail notions such as causation, change, or other notions that on this analysis are assumed to be templatic, including in particular the two result states entailed by the $\mathrm{P}_{\text {have }}$ and $\mathrm{P}_{\text {loc }}$ heads, putatively possession and co-location respectively.

Nonetheless, we argue once again that the roots here can entail template-like thematic and aspectual meaning. In particular, we suggest that some roots of ditransitive verbs bring to bear change, possession, and co-location above and beyond what is already entailed by the two templates, sometimes in ways that have grammatical significance. Partly the evidence for this is that the templatic interpretation of each construction is actually determined by the choice of root, the verb sensitivity hypothesis of Rappaport Hovav and Levin (2008), which we relabel here the "root sensitivity" hypothesis since we assume it boils down to the root that occurs within each template. This suggests that the templates in this case are actually underspecified for certain templatic information, and it is the root that fills this information in. ${ }^{4}$ The resultant analysis derives a typology of roots that in turn therefore determines a verb class typology that crosscuts, but crucially also interacts with, the two templates that such roots occur in. This suggests a role for roots in a predictive theory of verb classes, which we discuss further in $\$ 5.4$.

We begin in $\$ 3.2$ with a brief overview of the ditransitive verbs that are of interest to us here and the history of treating possession and co-location and changes into those notions as templatic in nature. We then provide in $\$ 3.3$ an argument for the underspecification of the templates and the effect of roots on

${ }^{3}$ Indeed, even lexicalist event structures such as those proposed by Pinker (1989: 210-23), Jackendoff (1990b: 135-7), and Van Valin and LaPolla (1997: 102-28) have involved broad semantic primitives embodying notions such as possession and also structural asymmetries between the two arguments that differ across the two templates in ways that ultimately capture the same fundamental intuitions (see also Beavers et al. 2009: 111-21).

${ }^{4}$ This conclusion is in fact presaged in Harley and Jung (2015: 716, fn. 18) (building also on Beavers 2011a), who propose that the manner adjunction operation composing roots with $v_{\text {cause }}$ must ensure that root meanings can sometimes strengthen the template meaning, exactly the technical solution we develop in detail below. 
the templates, before turning to an argument that roots also entail possession, co-location, and change, evidenced by their effects on the telicity of ditransitive predicates, which we discuss in $\$ 3.4$. We then present a formal analysis of the meanings of roots and templates that can account for this in $\$ 3.5$, positing four broad subclasses of ditransitive verbs in $\$ 3.6$ based on the types of templatic information they entail, a refinement of the classification in Beavers (2011a: 44, Fig. 1). We support these conclusions by arguing that roots have templatic thematic information significant enough to derive some facts about which verbs are durative and punctual in $\$ 3.7$, and also which verbs show both variants of the dative alternation in $\$ 3.8$. We then present some alternative analyses in $\$ 3.9$ that are consistent with Bifurcation and show that they are inadequate in various ways. We support the continued need for templates in an analysis of ditransitive meaning despite the roots effectively determining the bulk of the verb’s meaning in $\$ 3.10$. Again, as with change in the roots of change-of-state verbs, the key question that arises from these results is why the roots of ditransitives would entail these various templatic notions. We ultimately suggest that this largely has to do with the kinds of actions they describe, which we propose are conceived of as inherently giving rise to changes in possession and location in ways that necessarily require Bifurcation to be violated. However, we defer a full discussion of this to $\$ 5.2$, instead focusing here just on making the case that the roots of ditransitives do violate Bifurcation.

\subsection{Ditransitive verbs, possession, and co-location}

We first establish the classes of verbs we are interested in. Pinker (1989: 110-23) (building on Green 1974: 110-33) identifies several subtypes of ditransitive verbs, including those in (6) and (7) (summarized from Gropen et al. 1989: 2434). Following Beavers (2011a), for our purposes we focus on only the verbs in boldface, the ones that describe the potential possession or co-location of physical or electronic objects. The verb classes we set aside are primarily verbs of communication, which do not (necessarily) describe transfer of tangible objects. It is certainly possible to give them an analysis where the communicated message is analyzed as a metaphorical physical object that the recipient comes to possess, although this is not the only possible way to analyze these data. For example, Tenny (1994: 17-9), Ramchand (2008: 25-33), and Rappaport Hovav (2008: 24-5) analyze such verbs as path object verbs akin to John walked the plank, treating the subject as the theme and the direct object as a metaphorical path. For now we leave the question of what the right analysis for these is aside and focus on the verbs that are more clearly used to express caused possession and caused co-location. ${ }^{5}$

\footnotetext{
${ }^{5}$ Since our interest here is in caused possession, we ignore light verb uses that some of these verbs have such as give someone a kiss or pay someone a visit. We also set aside a small set of verbs that have indirect objects but not always to variants which are entailed lose or lack of possession, such as deny,
} 
(6) Verbs allowing an indirect object:

a. Verbs that inherently signify acts of giving: give, pass, hand, sell, pay, trade, lend, loan, serve, feed

b. Verbs of sending: send, mail, ship

c. Verbs of instantaneous causation of ballistic motion (Verbs of throwing): throw, toss, flip, slap, kick, poke, fling, shoot, blast

d. Verbs of continuous causation of accompanied motion in a deictically specified direction: bring, take

e. Verbs of future having: offer, promise, bequeath, leave, refer, forward, allocate, guarantee, allot, assign, allow, advance, award, reserve, grant

f. Verbs of type of communicated message: tell, show, ask, teach, pose, write, spin, read, quote, cite

g. Verbs of instrument of communication: radio, email, telegraph, wire, telephone, netmail, fax

(7) Verbs supposedly not allowing an indirect object:

a. Verbs of fulfilling: credit, present, entrust, supply, trust

b. Verbs of continuous causation of accompanied motion in some manner: carry, pull, push, schlep, lift, lower, haul

c. Verbs of manner of speaking: shout, scream, murmur, whisper, shriek, yodel, yell, bellow, grunt, bark

d. Verbs of communication of propositions and propositional attitudes: say, assert, question, claim, think aloud, doubt

Partly the classes in (6) and (7) are motivated on an intuitive semantic basis, depending on the type of real-world semantics described by the root (e.g. different ways of sending or otherwise causing something to go to or become possessed by something else) and partly by the interpretation of the result state in either or both variants. We focus on this latter distinction below, ultimately proposing that these roots can be reclassified into a principled typology of root types according to this latter factor (building on the typology of verbs proposed in Beavers 2011a: 44, Fig. 1). As part of arguing for this we also establish more carefully exactly what the meanings of the templates are, suggesting that "caused possession" and "caused colocation" are too specific to capture the full range of interpretations of various roots

cost, or spare, since their semantics is especially unique (for more on these see Bruening 2010a,b; Harley and Jung 2015, and Harley and Miyagawa 2017: 16-7). There is also an additional pattern for verbs of caused possesion involving with PPs, as exemplified by supply in supply arms to the troops vs. supply the troops with arms vs. supply the troops arms. This presumably involves yet another template, but not one that we address here. 
in each construction (recapping Rappaport Hovav and Levin 2008 and Beavers 2011a).

First, we briefly establish that possession and co-location are templatic notions at all independent of the fact that they are posited as being introduced by templatic heads in (3). That possession can be analyzed as a basic, templatic semantic primitive has a long history dating back at least to Green (1974: 133-54), McCawley (1974), Ross (1976), and Dowty (1979: 269-71), inter alia, as a subcomponent of words like have, get, and want, much of the argumentation based on sublexical scopal facts (see also Richards 2001, Harley 2003, Harley 2004, and Bruening 2010a,b regarding argumentation based on argument asymmetries and idiom formation; see Beavers et al. 2009: 113-21 for an analysis of possession as a basic universal lexical entailment in a non-decompositional framework). For example, the temporal modifier for an hour modifying a predicate headed by want can either describe the duration of the wanting or the duration of the implicit having, suggesting a distinct, modifiable "have"-denoting predicate somewhere in the verb’s event structural decomposition (as per $\$ 1.3 .2$; for more on sublexical modification see $\$ 3.9$ ):

(8) John wanted the car for an hour.

Thus the existence of a possessional templatic element of some kind is widely accepted and not that controversial.

A secondary question is what exactly the semantics of possession is, and this is a murkier issue. As noted by Beavers et al. (2009: 109-10) most if not all of the constructions that have been assumed to contain the relevant templatic operator systematically admit the same sets of possessive readings (modulo effects of particular roots in ruling in or out certain types of possession). For example, give admits the four types of possession identified by Tham (2006: 138-9), including caused inalienable possession (e.g. as with relational noun possessees; see also Partee 1999) and alienable possession (e.g. as with sortal noun possessees), plus what Tham calls "control" possession where the recipient comes to have control over the possessee for some period of time even if not (in)alienably possessing it, and "focus" possession, where the recipient comes to be related to the theme by some contextually defined relationship even if not (in)alienably possessing it or controlling it, a feature shared with have, get, and also adnominal possessive constructions (Barker 1995; Vikner and Jensen 2002). These readings are illustrated for give in (9) (taken from Beavers et al. 2009: 110, (7)):

(9) a. John gave Bill a black eye.

b. John gave Bill a car.

c. John gave Bill the car (for the weekend).

d. John gave Bill the windows (to clean). (inalienable possession) (alienable possession) (control possession) (focus possession) 
Below, it will usually not be especially important to distinguish between these notions of possession. Our goal rather is to demonstrate that possession of some type or another comes from either a root or a templatic head, and that ditransitives sometimes (though not always) describe a change in some possession relationship or another between the theme and either the recipient or the causer. If a distinction between two or more types of possession does become relevant, we will clarify it as necessary.

Regarding co-location, Hale (1986: 239) defined a notion of "central coincidence" wherein "the center of the figure coincides with the center of the place" that subsequently figured into the L-syntactic lexical decompositions of Hale and Keyser (2002: 208). Similarly, lexical semantic templatic operators and functional heads (usually prepositions) with the semantics of co-location have figured into various analyses of motion constructions (see e.g. Inagaki 2001; Stringer 2001, 2005, 2006; Fábregas 2007; van Riemsdijk 2007; van Riemsdijk and Huijbregts 2007, inter alia; see also Dowty 1979: 207-19, who posits basic co-locational primitives in his lexicalist event structures). Thus this semantic relation has also been widely (if not necessarily universally) assumed to be templatic in nature.

Finally, the key claim of the Harley (2003) analysis of ditransitives is that in both variants of the dative alternation there is a change into a state of possession or colocation. Change is itself (as discussed in \$1.3) a templatic notion. Furthermore, the type of change involved is not any different from the type of change involved in canonical change-of-state verbs. Beavers $(2011 \mathrm{~b}, 2012)$ argues that motion and more canonical types of change-of-state are all amenable to exactly the same scalar analysis of change-of-state (see $\$ 1.6 .1$ ), and Beavers (2011a) provides an extensive argument for extending this analysis to changes in possession. In sum, possession, co-location, and changes into these states are well established cross-cutting templatic notions, and ditransitive verbs in both variants of the dative alternation thus represent a subtype of verbs of caused change-of-state. For our purposes, then, the question is what elements in the event structures of ditransitive verbs contribute the meanings of possession (of any type), co-location, and change-of-state, and in particular whether it is ever the root that does this.

\subsection{The meanings of the templates: A case for root sensitivity}

In this section we discuss the meanings of the two templates that define the dative alternation, arguing that each is much more underspecified than typically assumed in prior work, drawing heavily on earlier argumentation in Rappaport Hovav and Levin (2008) and Beavers (2011a) (see also Green 1974). The residual meaning of particular root+template combinations must therefore be coming from the root, sometimes in ways that make the two variants of the dative alternation semantically distinct and sometimes in ways that make them synonymous, i.e. the phenomenon of "root sensitivity." 


\subsubsection{The semantics of indirect object templates}

For indirect object constructions it is commonly assumed that the meaning of the template includes some notion of the indirect object coming to possess the direct object. In reality only a small number of verbs-Pinker's giving verbs in (6a) (e.g. give, hand, pass (salt)) - actually require possession to obtain. This is illustrated by the fact that the inference that the indirect object came to possess the theme is non-cancelable with these verbs in this construction:

(10) a. \#Mary gave John a ring, but he never got it.

b. \#Mary loaned John a book, but he never got it.

c. \#Mary passed John the salt, but he never got it.

However, the verbs in every other relevant class in (6) and (7)-including verbs of sending as in (11a), ballistic motion as in (11b), continuous motion as in (11c), future having as in (11d), and instrument of communication as in (11e)-do not require the possessor to actually come to possess the theme. Rather, this inference is cancelable in context, suggesting that if there even is such an inference it is at best a conversational implicature of some sort: ${ }^{6}$

(11) a. Mary sent/mailed John a letter, but it got lost in the mail and he never got it.

b. Mary threw/hurled/tossed John a ball, but it got blown off course and he never got it.

c. Mary brought/took John a letter, but forgot about it in her pocket and so he never got it.

d. Mary left/bequeathed John her inheritance, but then spent it all before he could get it.

e. Mary emailed/faxed John her manuscript, but the power went out at his office and he never got it.

Given that it is contingent on the root whether or not possession is actually entailed by the verb or merely implicated, actual possession must in at least some cases be coming from the root, not the template. Were actual possession entailed by the template then all uses of the indirect object template with all roots should entail actual possession, contrary to fact.

${ }^{6}$ A reviewer asks whether (11d) might not involve an acquired inheritance, albeit one whose value is $\$ 0$. Given that cancelability is a general phenomenon for ditransitives and that there is a natural understanding of this event as not inheriting anything, we maintain here that the inheritance in this case simply does not arise, though on the reviewer's interpretation this case could be subsumed with the verbs like give above. 
However, crucially, in all cases of the indirect object construction, prospective possession-the possibility of possession even if possession does not actually come about-is always entailed (Gropen et al. 1989: 207). This is evidenced by the fact that when the theme is somehow incapable of possession (e.g. the theme is inanimate) the resulting predicate is infelicitous unless the indirect object is somehow reinterpreted as an inanimate that is capable of possession, i.e. even though possession is not required the London Office effect still obtains. Thus (12) represents sentences corresponding to the first clauses of (11) albeit with an inanimate indirect object London in all cases, and they are all infelicitous (or rather, require a London Office reading):

(12) a. \#Leah sent/mailed London a letter.

b. \#Leah threw/hurled/tossed London a ball.

c. \#Leah brought/took London a letter.

d. \#Leah left/bequeathed London her inheritance.

e. \#Leah emailed/faxed London her manuscript.

These facts are straightforwardly accounted for if we assume that while the indirect object template does not entail actual possession, it does entail prospective possession, and it is the presence of some shared possessional meaning in both actual and prospective possesion that accounts for these interpretations.

That said, an alternative analysis might simply be that the indirect object cannot be inanimate. However, this is not the case, since inanimates are quite natural as indirect objects if they are capable of possessing the given theme. In (13) the inanimate the wall is a fine (inalienable) possessor of the particular theme a fresh coat of paint, and no London Office effect arises:

(13) Kim gave the wall a fresh coat of paint.

Similarly, again, all of the sentences in (12) are acceptable if London is instead interpreted as the London Office, suggesting that it is not inanimacy per se that derives the semantic oddity of (12) but is instead something to do with ability to possess, consistent with an analysis where indirect objects are associated with the possibility of possession. Given that this occurs across all roots in the indirect object construction, it strongly suggests that the London Office effect is derived by a semantic property of the template and not the individual roots, easily explained if what the template entails is not caused possession but prospective caused possession.

Finally, when certain roots occur in the indirect object construction there is an additional interpretation that the theme undergoes a change of location (contra Krifka 2004: 17-19, who claims that indirect object constructions entail only caused possession and never caused motion). This is the case for hand, which 
requires that the theme end up physically located on the recipient's body (in addition to being actually possessed by the recipient):

(14) \#Mary handed John the ball, but it never left her hand.

Conversely, other roots require no change of location e.g. one can give, leave, or bequeath somebody something on paper without it actually being physically transferred anywhere:

(15) a. Sandy gave her son her collection of rare jewels, which continued to remain locked away in a safe deposit box.

b. Sandy left her son her collection of rare jewels, which continued to remain locked away in a safe deposit box.

c. Sandy bequeathed her son her collection of rare jewels, which continued to remain locked away in a safe deposit box.

If co-location is a templatic notion then it is obviously not coming from the event template in this case since it is not required for all constructions. Thus it must be coming from roots such as hand, and others we discuss below. In sum, the indirect object construction seems to entail prospective possession but not either actual caused possession or actual or prospective caused co-location. Any readings of actual caused possession or actual or prospective caused co-location arises owing to individual roots, meaning that these roots must be contributing these meanings to the overall meaning of the construction, contra Bifurcation.

\subsubsection{The semantics of to templates}

Turning now to the to construction, the standard understanding is that the underlying template entails caused change of location, and indeed, change of location is sometimes required by certain roots in this construction. This is the case with accompanied motion roots, both those with manner such as carry and haul and the deictic verbs bring and take:

(16) a. \#Kim carried/hauled the box to the council meeting, but it did not arrive.

b. \#Kim took/brought the box to the council meeting, but it did not arrive.

Crucially, however, with most roots in this construction arrival of the theme at the location indicated by the to PP is not required. This is the case above for give and verbs of future having in (15), and also with ballistic motion verbs such as throw and hurl. This is evidenced by the fact that arrival with this latter class of verbs 
can be canceled, regardless of whether the to PP is animate (and thus a natural recipient) or inanimate (and thus a natural goal):

(17) a. Kim threw/tossed the ball to Sandy, but the wind blew it into the bushes and so it never arrived.

b. Kim threw/tossed the ball to the other side of the courtyard, but the wind blew it into the bushes and so it never arrived.

That said, for some speakers arrival is at least prospective, i.e. there is an expectation that arrival be in principle possible. This is evidenced by the fact that in a context where there is some barrier preventing arrival of the theme altogetherand furthermore the causer is aware of this barrier-then the to construction with ballistic motion verbs is infelicitous: ${ }^{7}$

(18) [ Kim and Sandy are separated by an unbreakable glass wall that Kim is aware of. ]

\#Kim threw/tossed the ball to Sandy.

Although Kim's awareness of the barrier seems to facilitate the infelicity here, this does not mean that intentions or expectations on the part of the agent are necessarily part of the meaning. In a context where Kim throws a ball to Alex but meant for it to go to Sandy, Kim threw the ball to Alex is still felicitous (cp. Kim intended/tried to throw the ball to Alex, which is not felicitous in this context). ${ }^{8}$ That Kim's awareness matters may instead reflect the effect of Kim being mistaken about the situation, i.e. the acceptable reading of (18) when Kim is not aware of the wall is equivalent to Kim threw/tossed the ball to Sandy, not realizing this was not possible. Regardless of the explanation, the condition that rules out (18) is that arrival must be possible in some more basic sense than just what was intended or expected. Putting the pieces together, we might assume that the meaning of the to construction is prospective arrival, but on a root by root basis it can be actual arrival, which must therefore be coming from the root, again contra Bifurcation.

However, ascribing prospective arrival semantics to the to template is also not correct. Evidence for this comes from cases where certain roots in the to construction seem to only entail actual or prospective possession. In general, possession is not part of the meaning of the template, since there is no general

\footnotetext{
${ }^{7}$ By contrast, as expected, throw to is fine in such a context where the theme is an object that is known to be able to pass through barriers, as in (i).

(i) [ Kim and Sandy are separated by an unbreakable glass wall that Kim is aware of. ] Kim threw a kiss to Sandy.

${ }^{8}$ Furthermore, inanimate subjects are possible as well, as in The ball machine threw a tennis ball to Rachael, also ruling out that intentions are part of the meaning of throw.
} 
London Office effect with to constructions for all roots. For example, with sending verbs as in (19a), ballistic motion verbs as in (19b), and accompanied motion verbs without a manner as in (19c) and with a manner as in (19d) there is no such effect when the goal is inanimate:

(19) a. John sent/mailed the letter to London.

b. John tossed/hurled the ball to the edge of the field.

c. John brought/took the ball to the edge of the field.

d. John hauled/dragged the ball to the edge of the field.

However, crucially, with some roots the to construction does give rise to a London Office effect, as with roots of giving as in (20a) and future having as in (20b), just as they require in the indirect object template above:

(20) a. Mary gave/loaned/handed/passed the salt to Kim/\#London.

b. Mary bequeathed/left the money to Kim/\#London.

Yet, as argued above based on (15), these roots do not entail arrival or any kind of motion at all, unlike (say) ballistic motion verbs as illustrated in (18). Taken together, the examples in (20) are in fact exact thematic paraphrases of their indirect object variants in (21) (modulo non-truth conditional effects whereby the choice of variants is sometimes conditioned on making heavy XPs occur later in a sentence or XPs expressing given information occur earlier in the sentence; Wasow 2002; Bresnan and Nikitina 2009):

(21) a. Mary gave/loaned/handed/passed Kim/\#London the salt.

b. Mary bequeathed/left Kim/\#London the money.

That there is no arrival-or prospective arrival-for one set of ditransitive roots that may occur in this template strongly suggests that the meaning of the to construction is weaker than either (prospective) arrival or (prospective) possession, but compatible with either reading, since these are the two readings that arise with this construction with roots that otherwise show the dative alternation. It is contingent on the root which reading the construction takes.

Alternatively, one might argue-as Jackendoff (1996: 330) does (building on Gruber 1965) - that the cases in (15) and (20) do involve a notion of (prospective) arrival, but the arrival in this case is not along a physical path but rather a more abstract, metaphorical "possessional path," i.e. a path consisting of possessors of the theme as locations. In this case the theme "moves" in a metaphorical sense from one possessor to the other, possibly saving the analysis that there is always some type of arrival in the to construction. As Rappaport Hovav and Levin (2008: 138-42) argue in detail, this makes the incorrect prediction that at the beginning of 
the event the theme would have to be at or with some initial possessor, presumably the causer. However, there is crucially no initial possession in at least some cases of possessional readings with certain roots. This is illustrated in (22) with give, where neither the subject nor anyone else need initially possess the theme. Instead the reading is simply that the recipient comes to have the theme at the end of the event, which is a feeling in the case of the creeps, a coat of paint created over the course of the event in the case of a fresh coat of paint, and a thought or inspiration in the case of a book idea:

(22) a. John gave Mary the creeps.

b. John gave the wall a fresh coat of paint.

c. John gave Mary a book idea.

Indeed, in $(22 \mathrm{a}, \mathrm{c})$ the subject need not even do anything to cause the recipient to come to have the theme. Rather, in each case the subject the mere sight of John would be acceptable as well, a DP describing a referent that is incapable of possessing or even acting. Similarly, in $(22 \mathrm{a}, \mathrm{c})$ it is not necessarily the case that the creeps or the idea even existed prior to the event, further reinforcing that the agent need not have possessed anything. Alternatively, in some cases the agent may possess the theme, but the act of caused possession may not involve transfer but rather creation of a new such entity, as in Kim gave Sandy a cold where Kim still has a cold after the event but now there is a new cold which Sandy has (see also $\$ 3.6 .4)$. Altogether these data argue against somehow treating caused possession as abstract motion.

\subsubsection{Summary}

In sum, the real results entailed by the two templates are prospective possession for the indirect object template and prospective possession or co-location, which we refer to here as prospective $R^{\prime}$, a relation that covers both possession and co-location, for the to template. These are the only meanings that seem to be shared across all uses of the template respectively, regardless of the specific verbal root that occurs in it:

(23) a. Indirect object template meaning: Prospective caused possession
b. To template meaning: Prospective caused $R^{\prime}$

This raises the possibility that the two templates in some cases can describe exactly the same event, e.g. a caused possession event is compatible with both templates, while in other cases they diverge, e.g. something that lacks caused possession but involves caused motion can be described by a to construction but not the 
indirect object construction. From this we derive that barring lexical idiosyncrasy any indirect object template should have a corresponding to template but not necessarily vice versa, as discussed previously by Pesetsky (1995: 141), Rappaport Hovav and Levin (2008: 150-60), and Beavers (2010: 854-5) (and contra Harley 2003: 41-2), and furthermore that a given use of the indirect object template will entail the corresponding use of the to template. Two reviewers ask if this aligns with an observation dating back to at least Oehrle (1976) that some indirect object uses do not have corresponding to uses. Thus while it is possible to say Nixon gave Mailer a book in the sense that Richard Nixon inspired Norman Mailer to write a book, it is odd to say \#Nixon gave a book to Mailer on the same sense.

However, as noted by Snyder (2003: 34-43) and Rappaport Hovav and Levin (2008: 150-2), there is in fact no problem with getting the same reading of the to variant with give, provided the recipient DP is heavy as in Nixon's behavior gave an idea for a book to every journalist living in New York City in the 1970s (Snyder 2003: 35, (48)). Thus the asymmetry in the original examples does not indicate a categorical constraint, but perhaps instead a preference for using the indirect object frame for (idiomatic) pure caused possession meanings since this is the canonical use of the indirect object template across roots. This preference in turn blocks use of the to template for the same meaning even if the to template allows the same reading, unless factors like heaviness override that preference (see e.g. Beavers and Nishida 2010: 225-26). In sum, there is no reason to assume that the indirect object template has any meaning not expressible in the to template.

Crucially, though, as we have seen above, the choice of root can flesh out the meaning of the overall construction to involve more specific notions of possession or arrival. In some cases the root choice obliterates the distinction between the two variants of the dative alternation if the root is associated with actual or prospective possession (as with giving and future having roots) and in other cases it does not if it is not associated with any notion of possession itself (as with many roots that require a motion reading of some sort). Thus the dative alternation with send is semantically contentful but not with bequeath:

(24) a. John sent a book to Mary/London.

$\neq$ John sent Mary/\#London a book.

b. John bequeathed his fortune to Mary/\#London. = John bequeathed Mary/\#London his fortune.

This is the core contrast that motivated root sensitivity as per Rappaport Hovav and Levin (2008). Furthermore, these facts also demonstrate that the roots of future having verbs entail exactly the meaning entailed by the indirect object template-prospective possession-and the roots of giving verbs entail something even stronger-actual receiving-that entails prospective possession, meaning 
that by entailment these roots also entail this. Hand and ballistic and accompanied motion roots seem to entail possible or actual arrival. Since both (prospective/actual) possession and (prospective/actual) arrival are compatible with the prospective $R^{\prime}$ state that is at the core of to templates, then again by entailment those roots all entail that notion as well. But since (prospective) possession and (prospective) $R^{\prime}$ are both templatic notions then by virtue of entailing these two notions all ditransitive roots thus constitute counterexamples to Bifurcation. We next turn to some additional templatic properties of the roots of ditransitives, focusing on their lexical aspectual contributions.

\subsection{Change of state and aspectual properties: Root telicity}

In this section we look at another property that is fundamentally templatic in nature, namely the entailment of a change of state. It should be clear from the data in the previous section that at least some root plus template combinations give rise to entailments of change of state, e.g. the coming about of actual possession or co-location, even if not all such combinations do. However, this fact is at odds with another property of ditransitives, namely telicity. Recapping $\$ 1.6 .1 .1$, telicity is intuitively the property of a predicate over events where it names the culmination point of the events it describes, e.g. the coming about of a particular result state. More precisely, we adopted the definition of telicity in Beavers (2012: 34-5) as a property of a predicate over events where for any event in its denotation it does not describe any non-final subevent of that event. This permits a wider range of predicates to count as telic, including those that do not describe result states but do describe events that are temporally bounded in other ways (e.g. semelfactives on a non-iterative reading such as John blinked once; see \$1.6.2.2), though telicity as determined by the coming about of a result state at the end of the event will be of central interest here.

On many event decompositional approaches it is assumed that telicity as determined by result states is more specifically the outgrowth of particular templatic configurations (as noted by Folli and Harley 2004: 99). For example, among the class of verbs that describe caused changes broadly speaking, telicity supposedly arises from the coming about of a result described by the complement of $v_{\text {cause }}$ or $v_{\text {become }}$, which defines the endpoint of the event (see the analyses of accomplishments and achievements in Rappaport Hovav and Levin 1998: 108 and Ramchand 2008: 74-91, inter alia). ${ }^{9}$ Crucially, then, telicity for canonical changeof-state verbs is thus supposedly contingent on whatever result is entailed by the

${ }^{9}$ Recall also from $\$ 1.6 .1 .1$ that reference properties of the theme DP can also have an effect on telicity, where bare plurals and mass DPs ensure atelicity for all change-of-state verbs even if the result state is named. Since this contributing factor is orthogonal to our concerns here, we continue to use only theme DPs with quantized reference to control for it. 
verb's event template. The role of the root on standard approaches to the event structures of such verbs and thus to calculating telicity is that the root just describes the specific result entailed by the verb. But it does not itself actually entail the change.

Interestingly, however, as noted first by Jackendoff (1996: 330), all ditransitive predicates are telic, readily accepting in PP modifiers but being less acceptable with for PPs, regardless of which of the two templates is used:

(25) a. Sandy gave/loaned Alex a book in/?for an hour.

b. Sandy sent/mailed Alex a letter in/?for an hour.

c. Sandy threw/hurled/tossed Alex a ball in/?for an hour.

d. Sandy brought/took Alex a letter in/?for an hour.

e. Sandy left/bequeathed Alex his fortune in/?for an hour.

f. Sandy emailed/faxed Alex his manuscript in/?for an hour.

(26) a. Sandy gave/loaned a book to Alex in/?for an hour.

b. Sandy sent/mailed a letter to Alex in/?for an hour.

c. Sandy threw/hurled/tossed a ball to Alex in/?for an hour.

d. Sandy brought/took a letter to Alex in/?for an hour.

e. Sandy left/bequeathed his fortune to Alex in/?for an hour.

f. Sandy emailed/faxed his manuscript to Alex in/?for an hour.

The reading in most of these cases is that the event happend after an hour, suggesting that these predicates are all punctual (see $\$ 1.6 .1 .1$ on tests for punctuality and $\$ 3.7$ for more on punctuality with ditransitives). But punctual predicates are a subtype of telic predicates (Beavers 2012: 23), where the culminating event happens to be short; it is telicity that fundamentally yields acceptability for in an hour modifiers (since it is possible for both punctual and durative telic predicates; see $\$ 1.6 .1 .1$ and $\$ 1.6 .2 .2$ ). So taken altogether these data suggest that ditransitives are uniformly telic. But why would they be telic? If the assumption for ditransitives is that the root simply describes the action that led to the outcome of (prospective) caused possession or $R^{\prime}$, the prediction is that the root should not be implicated in telicity at all. Rather, the result state that will determine whether telicity arises should be the one described by the stative $\mathrm{P}$ heads of each template that project the complement of $v_{\text {cause }}$.

Yet, crucially, as argued in detail above, the result states represented by the templates of ditransitive verbs are merely prospective and not actually entailed to obtain. This means that any given ditransitive should be able to describe not just an event in which the template-defined outcome actually does obtain, but also non-final subevents of that event in which it does not obtain. Indeed, sometimes this is in fact exactly the case, as illustrated in the following for ballistic motion verbs: 
(27) Sandy threw the ball to Alex ...

a. and it got to her just fine. (a culminating event)

b. but it only got halfway there. (a non-final subevent of (27a))

c. but it only got one quarter of the way there.

(a non-final subevent of (27b))

(28) Sandy threw Alex the ball ...

a. and it got to her just fine.

(a culminating event)

b. but it only got halfway there.

c. but it only got one quarter of the way there.

(a non-final subevent of (28a))

(a non-final subevent of (28b))

In each case the event description characterized may be of some event $e$ involving actual arrival at the expressed goal but also a non-final subevent $e^{\prime}$ of $e$ lacking arrival at the goal, e.g. the theme travels halfway or a quarter of the way along the same path. Thus based solely on the templates, these predicates should be atelic according. What then derives their telicity?

Building on Beavers (2011a: 24-44) we suggest that what derives telicity is actually a root-specific non-prospective change of state above and beyond whatever prospective result is entailed by the template. In some cases they fill in the caused result entailed by the template but in others they contribute a wholly orthogonal change of state. This change of state furthermore arises from what should be a manner-describing root, meaning that root-entailed change of state coming from a state-describing root as seen in the previous chapter is not the only case of a root that can entail change of state. We refer to the phenomenon of roots giving rise to entailments that result in the predicate being telic "root telicity." We turn next to a formal analysis of roots and templates of ditransitive verbs that will provide a framework for exploring how each contributes to the meaning of a given ditransitive predicate, before going through a series of subclasses of roots and their entailments that will derive the root sensitivity and root telicity facts we have seen so far.

\subsection{Defining and composing template and root meanings}

We begin by sketching a compositional analysis of the meanings of the templates and roots of ditransitive verbs. We take as our starting point the analysis of Beavers (2011a), who argues extensively for a scalar analysis of ditransitive verbs (building specifically on Beavers 2011b, 2012; see \$1.6.1). However, Beavers does not provide a compositional analysis of ditransitives nor does he explain how the different meanings of the fully composed verbal predicates come to have the meanings they have based on some particular combinations of roots and templates 
(since he more generally does not assume an event structural framework), which is our goal. We thus develop those details here, arriving at a variant of Beavers's analysis (with modifications owing mostly to differences in the formal semantic framework).

Before we continue, we offer a clarification on the need for this formal analysis. Our argument above was that roots contribute templatic meaning, and this templatic meaning in turn combines with the actual meaning of the template the root occurs in to produce a variety of particular semantic effects in a way that sometimes subsumes the template's meaning, and at other times is augmented by it. This goes at odds to some degree with a typical understanding of a manner root in the sort of event structures we are adopting here as just classifying a type of action for one particular event within the larger template. For this reason we believe it would be useful to demonstrate that the typical formal semantic compositional tools usually assumed to define the truth conditions of event structures can in fact produce the effects we have demonstrated above. The actual analysis we propose is thus largely a proof-of-concept, and is only intended as such; there are presumably many ways to do this, some perhaps more elegant or insightful than what we propose below. We also do not fully analyze every aspect of the data, leaving some of the finer-grained details for future work. Our goal is to just give enough of a framework that the plausibility of such an analysis is demonstrated.

Note first, however, that in Harley's (2003: 32, (3)) event structures in (3) there are only two templatic heads $-v_{\text {cause }}$ representing causation and the two $\mathrm{P}$ heads representing the result states. There is no separate head introducing a become' semantics. This is not problematic-the meaning could simply be represented in one of the other two heads (e.g. built into $v_{\text {cause }}$ as per Harley 2012: 335-7 or into the $\mathrm{P}$ heads), or it could be that we need to assume a $v_{\text {become }}$ head below $v_{\text {cause }}$. The choice does not technically make any difference for our purposes, and so for simplicity we continue to maintain the structures exactly as in (3) and assume with Harley (2012) that the become semantics is in $v_{\text {cause }}$, but it is otherwise conjunctively a part of the ultimate meaning representation just as it would have been had we had a $v_{\text {become }}$ head. ${ }^{10}$

A somewhat more significant revision, though, is that we assume that the $v_{\text {cause }}$ head for ditransitives is not the same one as the one in previous chapters. In particular, recall above that the result states for many (if not all) ditransitives

${ }^{10}$ One place where the presence or absence of $v_{\text {become }}$ would matter is in defining possible attachment points for repetitive readings of again. Furthermore, as a reviewer notes, assuming manner roots do attach higher in the structure than the $\mathrm{P}$ head, if there is both a $v_{\text {cause }}$ and a $v_{\text {become }}$ head there is a question of which head the manner root attaches to, which will also have effects on the interpretation of repetitive modification. These are interesting questions, but ultimately repetitive modification is not our concern here, so we set these issues aside, though see some discussion of restitutive modification in $\$ 3.9$. 
are prospective, i.e. they can be canceled. This phenomenon is similar to that of non-culmination with transitive change-of-state verbs noted in \$1.6.2.1. As discussed there a common analysis in the literature is that for at least some types of non-culmination it reflects a modal operator by which while the causing event must occur at the evaluation world $w$, e.g. the real world $w_{0}$ in realis mood, the result does not necessarily obtain at $w$ but instead at some other world or set of worlds. Importing this assumption, we represent prospectivity by an existential sublexical modal operator $\diamond$ over the causation (see also Rappaport Hovav and Levin 2008: 135). It is not our goal here to fully work out the details of this modality, but since actual results are possible we assume the modal base of $\diamond$ includes the evaluation world $w$ and other worlds as well, where the relevant additional accessible worlds are some type of possible continuations (see Beck and Johnson 2004: 116, who specifically define such continuations as inertia worlds à la Dowty 1979: 134-5, even equating the modal with the PROG operator Dowty assumed underlies progressive aspect in English; see also Koenig and Davis 2001: 86-9 on agent intentions as the relevant modal base of the energetic modal they posit for ditransitives, though see $\$ 3.3 .2$ on why intentions may not be right). ${ }^{11}$ Given that this modality scopes over the caused change, it must be introduced when causation is introduced, and thus we assume it comes from the $v$ that introduces cause' (see e.g. Tatevosov 2008: 410; see also Folli and Harley 2004 more generally on flavors of $v$ ). We thus assume that the relevant causal head is the following:

$$
\begin{aligned}
& \llbracket v_{\diamond \text { cause }} \rrbracket=\lambda P \lambda x \lambda v\left[\operatorname { e f f e c t o r } ^ { \prime } ( x , v ) \wedge \diamond \exists e \left[\operatorname { c a u s e } ^ { \prime } ( v , e ) \wedge \exists s \left[\text { become }^{\prime}(s, e) \wedge\right.\right.\right. \\
& P(s)]]]
\end{aligned}
$$

The crucial point for our purposes is that there is an existential modal operator of some sort that scopes over the operators defining the caused coming about of the result state, ensuring that the result need not obtain even though the result semantics is a part of the meaning of certain predicates.

${ }^{11}$ We treat the modal as existential rather than universal, though Dowty's PROG operator was universal (also a common assumption in work on non-culmination, albeit for different reasons). Dowty's (pp. 147-9) justification came from cases with two possible but contradictory outcomes, as with flipping a coin. Here an existential modal would allow both It's coming up heads and It's coming up tails to be true simultaneously (since each is true in at least one possible inertia world), a counterintuitive result. By positing a universal modal both would be false, since each would hold of only some but not all inertia worlds (though this also strikes us as the wrong result since at least one is in fact true; perhaps it is better to say that the truth of each is indeterminate).

However, it is not obvious the same reasoning applies here. If Mary throws a ball to Alex and it goes to Maxine, both Mary threw a ball to Alex and Mary threw a ball to Maxine can be true. However, were there ultimately a reason to assume a universal modal the analysis we present below where roots will subsume templatic meaning is still possible. We assume that roots lexically entail changes into templatic states at the reference world, which is in the modal base of $\diamond$. On a universal modal analysis the root would lexically entail changes into templatic states at all worlds in that modal's modal base plus the reference world. In either case if the template's modalized change of state and the root-supplied change of state are identical the root will subsume the meaning of the template. That said, we leave a fully fleshed out analysis of the exact modality and its modal base to future work. 
The core meanings we will be interested in for the two P heads in (3) are possession and co-location. Consistent with $\$ 1.6 .1 .3$ we define these states in terms of scales. Following Beavers (2011a: 31), the scales appropriate for such verbs are either scales of possession, where the theme goes from not being possessed by the recipient to being possessed by it, scales of motion, where the theme goes from not being at some location to being at it, possibly via a series of adjacent intervening locations between its initial and final locations, and scales that admit both changes at once, plus occasionally other types of scales on a case-by-case basis. In the case of a path scale we assume that for every degree $d_{z}$ on the scale there is a corresponding spatial location $z$ that holding that degree on that scale corresponds to the theme being located at, where scalar adjacency corresponds to spatial adjacency in such a way that incremental progress along the scale corresponds to incremental progress through space. For simplicity we further assume that locations are distinct such that being at one precludes being at another. We assume pure possession scales are non-gradable, consisting of just two degrees, where the maximal degree $d_{z}$ corresponds to the theme being possessed by a possessor $z$ and the minimal degree corresponds to not being possessed by $z$. Note that in the minimal state there need not be another possessor; the final possessor simply does not possess the theme, i.e. the scale derives coming to possess, not transfer of possession (contra Jackendoff 1996: 330, as discussed in \$3.3.2), though in a particular context a scale of coming to possess could be interpreted as reflecting transfer of possession if conditions ensuring initial possession arise independently.

The intersection of path scales and possession scales represents both kinds of states simultaneously. These are effectively structured like path scales in that they define a series of locations, but for some degrees on the scale the theme is not just located at the corresponding location but also possessed by it, with a further constraint that at all degrees prior to that degree on the scale the given possessor does not possess the theme (and thus again there is no necessary path of possessors à la Jackendoff, though a path of possessors could be possible in context). ${ }^{12}$ These scales - plus the quantity of the theme-are related homomorphically to the change event in ways that predict the telicity facts discussed in $\$ 1.6 .1 .1$ by defining a unique final point of the event in terms of the unique final point on the scale in any given case.

The ultimate definitions for possession and location are given in (30):

(30) a. $a t^{\prime}(y, z, s)$ (" $y$ is at $z$ in $s$ ") is true iff there is a degree $d_{z}=\mathbf{d}_{x}^{\mathrm{p}}$ and $s$ is the state of $y$ holding degree $d_{z}$ on path scale $\mathbf{p}$.

b. have' $(y, z, s)$ (" $z$ has $y$ in $s$ ") is true iff there is a degree $d_{z}=\mathbf{d}_{x}^{\mathbf{p}}$ and $s$ is the state of $y$ holding degree $d_{z}$ on possession scale $\mathbf{p}$.

\footnotetext{
${ }^{12}$ Unlike the property scales assumed in the previous chapters, these scales are defined in terms of location or possession by specific real world entities and as such there are a multitude of such scales for all possible paths and possessors. For this reason we just represent the relevant scale by some constant $\mathrm{p}$ that is appropriate for the specific given state.
} 
The notion of possession defined here will form the core semantics of indirect object templates and certain root classes. However, recall that to templates entail something that generalizes over location and possession. We represent this by defining a relation $R^{\prime}$ defined disjunctively for all $y, z, e$ as in (31):

$$
R^{\prime}(y, z, e) \text { is true iff } \operatorname{have}^{\prime}(y, z, e) \text { is true or } a t^{\prime}(y, z, e) \text { is true. }
$$

We thus define the meaning of the two major result heads as follows:
a. $\llbracket \mathrm{P}_{\text {have }} \rrbracket=\lambda y \lambda z \lambda e\left[\operatorname{have}^{\prime}(y, z, e)\right]$
b. $\llbracket \mathrm{P}_{\mathrm{loc}} \rrbracket=\lambda z \lambda y \lambda e\left[R^{\prime}(y, z, e)\right]$

Absent a root, the meaning of the two templates themselves, when the heads in (29) and (32) are composed and applied to definite XPs of type $e$ whose meanings are $\mathbf{x}$ (causer), $\mathbf{y}$ (theme), and $\mathbf{z}$ (goal/recipient), will be (33): ${ }^{13}$

$$
\begin{aligned}
& \text { a. } \llbracket\left[{ }_{v \mathrm{P}} \mathrm{DP}_{x} v_{\diamond \text { cause }}\left[\mathrm{pP} \mathrm{DP}_{z}\left[\mathrm{p}_{\mathrm{p}^{\prime}} \mathrm{P}_{\text {have }} \mathrm{DP}_{y}\right]\right] \rrbracket\right. \\
& =\lambda v\left[\operatorname{effector}^{\prime}(\mathbf{x}, v) \wedge \diamond \exists e\left[\operatorname{cause}^{\prime}(v, e) \wedge \exists s\left[\text { become }^{\prime}(s, e) \wedge \operatorname{have}^{\prime}(\mathbf{y}, \mathbf{z}, s)\right]\right]\right] \\
& \text { b. } \llbracket\left[{ }_{v \mathrm{P}} \mathrm{DP}_{x} v_{\diamond \text { cause }}\left[\mathrm{PP} \mathrm{DP}_{y}\left[\mathrm{p}_{\mathrm{p}^{\prime}} \mathrm{P}_{\text {loc }} \mathrm{PP}_{z}\right]\right] \rrbracket\right. \\
& =\lambda v\left[\text { effector }^{\prime}(\mathbf{x}, v) \wedge \diamond \exists e\left[\text { cause }^{\prime}(v, e) \wedge \exists s\left[\text { become }^{\prime}(s, e) \wedge R^{\prime}(\mathbf{y}, \mathbf{z}, s)\right]\right]\right]
\end{aligned}
$$

The outcome then is that the two templates contribute prospective caused possession and prospective caused $R^{\prime}$ respectively.

The next question is what the meanings of the roots are and how they are combined into these templates. Crucially, ditransitive roots define a manner $M^{\prime}$ for causer $x$ plus also possibly information $Q^{\prime}$ about the templatic result predicate's theme $y$ and goal/recipient $z$. Given that we are assuming that the root adjoins to $v_{\Delta \text { cause }}$ it is easy to see how it can place constraints over the causer $x$ and/or causing event $v$, which have not yet been introduced or bound off at the point at which the root is adjoined to $v_{\diamond \text { cause }}$. But it is not as obvious how it will place constraints over $y$ and $z$ given that those will have composed with the relevant result predicate already. However, the manner root will have access to the result predicate itself, from which it is possible to determine the referents of these two arguments by picking out the specific unique individuals that bear these roles according to that predicate in event $e$. For this we define two functions $t h^{\prime}$ and $r g^{\prime}$ that take the result predicate $P$ and pick out the unique theme and result/goal respectively entailed by $P$, assuming uniqueness of participants as per Krifka (1998:

\footnotetext{
${ }^{13}$ Here and below we assume the to found in to constructions is semantically vacuous in terms of its truth-conditional contribution, and the to PP has the same semantic type as its DP complement, which we will take to be the type of individuals $e$.
} 
209, (41)) (see also Wechsler 2005b: 183-4 on using event variables for heads to pick out event participants selected by other heads): ${ }^{14}$
a. $\forall P\left[t^{\prime}(P)=\iota y \forall s \square\left[P(s) \rightarrow \exists z\left[R^{\prime}(y, z, s)\right]\right]\right]$
"For all $P, t^{\prime}(P)$ is the theme of any state described by $P$ "
b. $\forall P\left[r g^{\prime}(P)=\imath z \forall s \square\left[P(s) \rightarrow \exists y\left[R^{\prime}(y, z, s)\right]\right]\right]$
"For all $P, r g^{\prime}(P)$ is the recipient/goal of any state described by $P$ "

We will thus assume the root has a denotation that is the same type as $v_{\diamond \text { cause }}$, applying manner $M^{\prime}$ and information $Q^{\prime}$ over the relevant participants: ${ }^{15}$

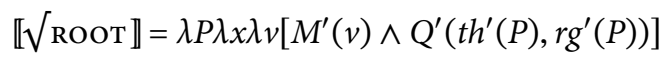

Finally, we assume that roots combine with $v_{\diamond \text { cause }}$ via head-adjunction, which yields the semantics in (36) (cp. Kratzer's 1996: 121-22 Event Identification) to produce a modified causal head meaning. (Here and below we underline the components of meaning introduced by the root so as to highlight which parts of the composed meaning are coming from where.)

$$
\begin{aligned}
& \llbracket\left[{ }_{v} v_{\diamond \text { cause }} \sqrt{\text { Rоот }] \rrbracket}=\lambda P \lambda x \lambda v\left[\llbracket v_{\diamond \text { cause }} \rrbracket(P, x, v) \wedge \llbracket \sqrt{\text { Rоот } \rrbracket(P, x, v)]}\right.\right. \\
& =\lambda P \lambda x \lambda v\left[\text { effector }(x, v) \wedge \diamond \exists e\left[\text { cause }^{\prime}(v, e) \wedge \exists s\left[\text { become }^{\prime}(s, e) \wedge P(s)\right]\right] \wedge\right. \\
& \underline{\left.M^{\prime}(v) \wedge Q^{\prime}\left(\text { th }^{\prime}(P), r g^{\prime}(P)\right)\right]}
\end{aligned}
$$

Applying (36) to predicates projected by $(32 \mathrm{a}, \mathrm{b})$ derives the templatic meanings in (37) for specific causer $\mathbf{x}$, theme $\mathbf{y}$, and recipient/goal $\mathbf{z}$ :

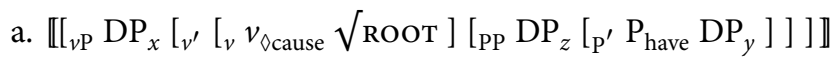

$$
\begin{aligned}
& =\lambda v\left[\text { effector }^{\prime}(\mathbf{x}, v) \wedge \diamond \exists e\left[\operatorname{cause}^{\prime}(v, e) \wedge \exists s\left[\text { become }^{\prime}(s, e) \wedge \operatorname{have}^{\prime}(\mathbf{y}, \mathbf{z}, s)\right]\right] \wedge\right. \\
& \left.M^{\prime}(v) \wedge Q^{\prime}\left(\operatorname{th}^{\prime}\left(\lambda s\left[\operatorname{have}^{\prime}(\mathbf{y}, \mathbf{z}, s)\right]\right), \operatorname{rg}^{\prime}\left(\lambda s\left[\operatorname{have}^{\prime}(\mathbf{y}, \mathbf{z}, s)\right]\right)\right)\right] \\
& \text { b. } \llbracket\left[{ }_{v \mathrm{P}} \mathrm{DP}_{x}\left[v^{\prime}\left[{ }_{v} v_{\diamond \text { cause }} \sqrt{\mathrm{ROOT}}\right]\left[\mathrm{PP}_{\mathrm{PP}} \mathrm{DP}_{\mathrm{P}^{\prime}} \mathrm{P}_{\text {loc }} \mathrm{PP}_{z}\right]\right]\right] \rrbracket \\
& =\lambda v\left[\text { effector }^{\prime}(\mathbf{x}, v) \wedge \diamond \exists e\left[\operatorname{cause}^{\prime}(v, e) \wedge \exists s\left[\text { become }^{\prime}(s, e) \wedge R^{\prime}(\mathbf{y}, \mathbf{z}, s)\right]\right] \wedge\right. \\
& \left.M^{\prime}(v) \wedge Q^{\prime}\left(t^{\prime}\left(\lambda s\left[R^{\prime}(\mathbf{y}, \mathbf{z}, s)\right]\right), r g^{\prime}\left(\lambda s\left[R^{\prime}(\mathbf{y}, \mathbf{z}, s)\right]\right)\right)\right]
\end{aligned}
$$

\footnotetext{
${ }^{14}$ One complication with the assumption of there being a unique participant satisfying each role is in the case of plural or quantificational DPs and/or pluralities of events. However, we assume plural DPs denote individuals representing mereological sums (see e.g. Krifka 1989; Link 2002), and otherwise that quantifiers are introduced above or raise to at least the highest $v \mathrm{P}$, above where (34) apply. Thus (34) are operating under the scope of any quantifiers and for any given individual bound variable under consideration it will be unique. (See Schein 1993 for a broader discussion of quantification and plurals of events and individuals.)

${ }^{15}$ The effector participant $x$ is treated as an argument of roots to make the array of arguments identical to those of $v_{\diamond \text { cause }}$, but is vacuously bound. This is not problem formally, and we furthermore leave open the possibility that perhaps roots do say something directly about the effector as part of defining the manner, though for now we simply treat the manner as a set of conditions on the causing event $v$.
} 
Note that (37b) is strictly more general than (37a) for the same root: the only real difference in meaning between them is that the former describes a possible $R^{\prime}$ outcome and the latter a possible having. But since any state of affairs that is a having is also an $R^{\prime}$ by (31), then the prediction is that anything that can be described in the indirect object construction can be described by the to construction but not conversely (see \$3.3.3). With this formal framework in place, we next show that root sensitivity and root telicity arise from the idiosyncratic information $Q^{\prime}$ that roots introduce into the predicate meaning, which will in many cases involve notions of possession, location, and change-of-state, among other things.

\subsection{Root classes}

We outline here four major root classes and some of their subtypes in terms of what kinds of result entailments they bring to bear within the entire predicate.

\subsubsection{True possession roots}

We refer to the first broad class as "true possession roots," which all share with one another that they entail actual caused possession of the theme by the recipient. The first subtype of these is what we call "pure caused possession" roots, which entail solely that and not much else. Give is the canonical example (and perhaps the only one with such a bleached semantics). As noted, it entails actual possession in both constructions:

(38) a. Mary gave John the book, \#but he never got it.

b. Mary gave the book to John, \#but he never got it.

As argued above, the semantics of actual possession must be coming from the root. We thus propose that the root $\sqrt{\text { GIVE }}$ has the denotation in (39). ${ }^{16}$

\footnotetext{
${ }^{16}$ Here and below we represent manner meanings for any verb V by assuming some predicate Ving' over the causing event $v$, largely ignoring the irrelevant idiosyncratic content of such manners (though see $\$ 5.2$ for some discussion of their content and its importance). In the case of give it may be that the giving' manner is minimal or empty (a point we return to in \$5.4). Note that in a few cases it may be that the relevant manner is also predicated of the theme argument as well (e.g. if it must move in a particular way; see Rappaport Hovav and Levin 1998: 113 for actions predicated of both causer and theme). This could be easily captured by assuming the root also stipulates something manner-oriented about the theme it has access to, e.g. as part of $Q^{\prime}$. For simplicity we ignore this extra aspect of meaning here. Finally, although we are assuming that manner is part of the meanings of the roots, we have not fully justified this. We return to this in $\$ 4.4 .3$.
} 


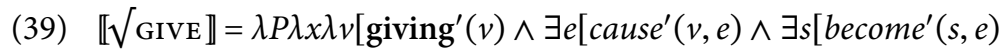
$\wedge^{\left.\left.\left.\operatorname{have}^{\prime}\left(\operatorname{th}^{\prime}(P), \operatorname{rg}^{\prime}(\bar{P}), s\right)\right]\right]\right]}$

Assuming the templatic meanings defined in the previous section, plus the DP denotations in (40) and the overall templatic structures in (3) (with $\sqrt{\text { GIVE }}$ in

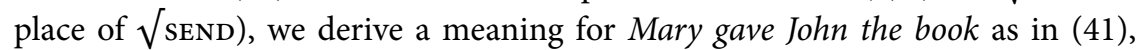
equivalent to substituting the specific lexical meanings and individual constants into the indirect object construction in (37a):

(40) a. $\llbracket$ Mary $\rrbracket=\mathbf{m}$

b. $\llbracket J o h n \rrbracket=\mathbf{j}$

c. $\llbracket$ the book $\rrbracket=\mathbf{b}$

(41) a. $\llbracket \mathrm{P}_{\text {have }}$ the book $\rrbracket=\lambda y \lambda z \lambda s\left[\right.$ have $\left.^{\prime}(y, z, s)\right](\mathbf{b})$

$=\lambda z \lambda s\left[\right.$ have $\left.^{\prime}(\mathbf{b}, z, s)\right]$

b. $\llbracket J o h n\left[\mathrm{P}_{\text {have }}\right.$ the book $] \rrbracket=\lambda z \lambda s\left[\right.$ have $\left.^{\prime}(\mathbf{b}, z, s)\right](\mathbf{j})$ $=\lambda s\left[\right.$ have $\left.^{\prime}(\mathbf{b}, \mathbf{j}, s)\right]$

c. $\left.\llbracket v_{\diamond \text { cause }} \sqrt{\mathrm{GIVE}} \rrbracket=\lambda P \lambda x \lambda v \llbracket \llbracket v_{\diamond \text { cause }} \rrbracket(P, x, v) \wedge \llbracket \sqrt{\mathrm{GIVE}} \rrbracket(P, x, v)\right]$

$=\lambda P \lambda x \lambda v\left[\lambda P \lambda x \lambda v\left[\right.\right.$ effector $^{\prime}(x, v) \wedge \diamond \exists e\left[\right.$ cause $^{\prime}(v, e) \wedge$

$\exists s\left[\right.$ become $\left.\left.\left.^{\prime}(s, e) \wedge P(s)\right]\right]\right](P, x, v) \wedge \lambda P \lambda x \lambda \nu\left[\right.$ giving $^{\prime}(v) \wedge$

$\exists e\left[\operatorname{cause}^{\prime}(v, e) \wedge \exists s\left[\right.\right.$ become $\left.\left.\left.\left.^{\prime}(s, e) \wedge \operatorname{have}^{\prime}\left(\operatorname{th}^{\prime}(P), \operatorname{rg}^{\prime}(P), s\right)\right]\right]\right](P, x, v)\right]$

$=\lambda P \lambda x \lambda v\left[\right.$ effector $(x, v) \wedge \diamond \exists e\left[\right.$ cause $^{\prime}(v, e) \wedge \exists s\left[\right.$ become $\left.\left.^{\prime}(s, e) \wedge P(s)\right]\right] \wedge$ $\operatorname{giving}^{\prime}(v) \wedge \exists e\left[\operatorname{cause}^{\prime}(v, e) \wedge \exists s\left[\right.\right.$ become $\left.\left.\left.^{\prime}(s, e) \wedge \operatorname{have}^{\prime}\left(\operatorname{th}^{\prime}(P), \operatorname{rg}^{\prime}(P), s\right)\right]\right]\right]$

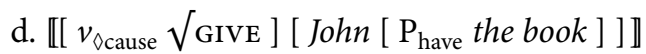

$=\lambda P \lambda x \lambda v\left[\right.$ effector $^{\prime}(x, v) \wedge \diamond \exists e\left[\right.$ cause $^{\prime}(v, e) \wedge \exists s\left[\right.$ become $\left.\left.^{\prime}(s, e) \wedge P(s)\right]\right] \wedge$ giving $^{\prime}(v) \wedge \exists e\left[\right.$ cause $^{\prime}(v, e) \wedge \exists s\left[\right.$ become $^{\prime}(s, e) \wedge$

$\left.\left.\left.\operatorname{have}^{\prime}\left(\operatorname{th}^{\prime}(P), r g^{\prime}(P), s\right)\right]\right]\right]\left(\lambda s\left[\operatorname{have}^{\prime}(\mathbf{b}, \mathbf{j}, s)\right]\right)$

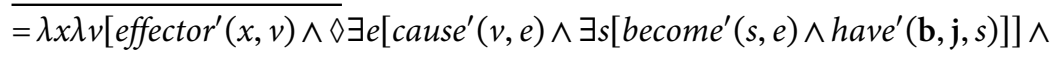
giving $^{\prime}(v) \wedge \exists e\left[\right.$ cause $^{\prime}(v, e) \wedge \exists s\left[\right.$ become $^{\prime}(s, e) \wedge$ $\left.\left.\left.\left.\overline{\operatorname{have}^{\prime}\left(\operatorname{th}^{\prime}\left(\lambda_{s}\left[\operatorname{have}^{\prime}(\mathbf{b}, \mathbf{j}, s)\right]\right), r g^{\prime}\left(\lambda_{s}\left[\operatorname{have}^{\prime}(\mathbf{b}, \mathbf{j}, s)\right]\right)\right.}, s\right)\right]\right]\right]$

e. $\llbracket \operatorname{Mary}\left[\left[v_{\diamond \text { cause }} \sqrt{\mathrm{GIVE}}\right]\left[\mathrm{John}\left[\mathrm{P}_{\text {have }}\right.\right.\right.$ the book $\left.\left.]\right]\right] \rrbracket$ $=\lambda x \lambda v\left[\right.$ effector $^{\prime}(x, v) \wedge \diamond \exists e\left[\operatorname{cause}^{\prime}(\nu, e) \wedge \exists s\left[\right.\right.$ become $\left.\left.^{\prime}(s, e) \wedge \operatorname{have}^{\prime}(\mathbf{b}, \mathbf{j}, s)\right]\right] \wedge$ $\operatorname{giving}^{\prime}(v) \wedge \exists e\left[\operatorname{cause}^{\prime}(v, e) \wedge \exists s\left[\right.\right.$ become $^{\prime}(s, e) \wedge$ $\left.\left.\left.\left.\overline{\operatorname{have}^{\prime}\left(\operatorname{th}^{\prime}\left(\lambda_{s}\left[\operatorname{have}^{\prime}(\mathbf{b}, \mathbf{j}, s)\right]\right), r g^{\prime}\left(\lambda s\left[\operatorname{have}^{\prime}(\mathbf{b}, \mathbf{j}, s)\right]\right)\right.}, s\right)\right]\right]\right](\mathbf{m})$ $=\lambda v\left[\right.$ effector $^{\prime}(\mathbf{m}, v) \wedge \diamond \exists e\left[\right.$ cause $^{\prime}(v, e) \wedge \exists s\left[\right.$ become $^{\prime}(s, e) \wedge$ have $\left.\left.^{\prime}(\mathbf{b}, \mathbf{j}, s)\right]\right] \wedge$ giving $^{\prime}(v) \wedge \exists e\left[\right.$ cause $^{\prime}(v, e) \wedge \exists s\left[\right.$ become $^{\prime}(s, e) \wedge$ $\left.\left.\left.\left.\overline{\operatorname{have}^{\prime}\left(\operatorname{th}^{\prime}\left(\lambda s\left[\operatorname{have}^{\prime}(\mathbf{b}, \mathbf{j}, s)\right]\right), \operatorname{rg}^{\prime}\left(\lambda s\left[\operatorname{have}^{\prime}(\mathbf{b}, \mathbf{j}, s)\right]\right)\right.}, s\right)\right]\right]\right]$ 
Ignoring tense and grammatical aspect, and assuming existential quantification over the event occurs at TP, we derive a final meaning in (42):

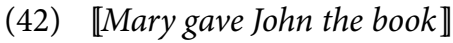

$=\exists v\left[\right.$ effector $^{\prime}(\mathbf{m}, v) \wedge \diamond \exists e\left[\operatorname{cause}^{\prime}(v, e) \wedge \exists s\left[\right.\right.$ become $^{\prime}(s, e) \wedge$

$\left.\left.\operatorname{have}^{\prime}(\mathbf{b}, \mathbf{j}, s)\right]\right] \wedge$ giving $^{\prime}(v) \wedge \exists e\left[\right.$ cause $^{\prime}(v, e) \wedge \exists s\left[\right.$ become $^{\prime}(s, e) \wedge$

$\left.\left.\left.\operatorname{have}^{\prime}\left(\operatorname{th}^{\prime}(\lambda s[\operatorname{have}(\mathbf{b}, \mathbf{j}, s)]), \operatorname{rg}^{\prime}\left(\lambda s\left[\operatorname{have}^{\prime}(\mathbf{b}, \mathbf{j}, s)\right]\right), s\right)\right]\right]\right]$

Substituting the outputs of $t h^{\prime}$ and $r g^{\prime}$ (b and $\mathbf{j}$ in this context), (42) is equivalent (43):

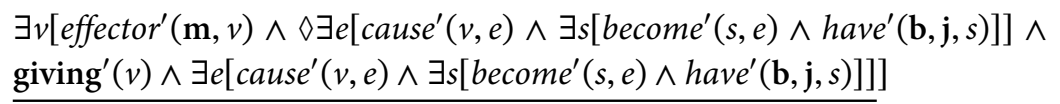

Since actual receiving entails prospective receiving (i.e. $P$ entails $\diamond P$ in this case, so that $[\nabla P \wedge P]$ is equivalent to $P$ ), then (43) is equivalent to (44), deriving that the meaning of the sentence is that there is some event with Mary as the causer whereby Mary caused by virtue of a giving manner that John actually came to possess the ball:

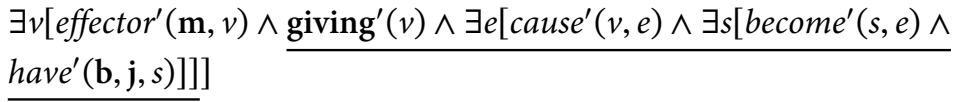

Key here is that the result entailed by the root is strictly stronger than that of the indirect object template, and thus the root monotonically strengthens what would otherwise be entailed by the template from possible to actual having.

Furthermore, the result entailed by the root also subsumes that of the to template as well (since for all $y, z, s h a v e^{\prime}(y, z, s)$ entails $R^{\prime}(y, z, s)$ ). Thus analogously to above, the meaning of Mary gave a book to John will ultimately resolve to the same meaning as in (44) (we omit compositional details from here on out, which in all cases will be entirely analogous to (41)):

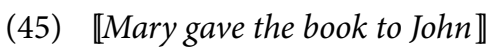

$=\exists v\left[\right.$ effector $^{\prime}(\mathbf{m}, v) \wedge \diamond \exists e\left[\operatorname{cause}^{\prime}(v, e) \wedge \exists s\left[\right.\right.$ become $\left.\left.^{\prime}(s, e) \wedge R^{\prime}(\mathbf{b}, \mathbf{j}, s)\right]\right] \wedge$

$\operatorname{giving}^{\prime}(v) \wedge \exists e\left[\right.$ cause $^{\prime}(v, e) \wedge \exists s\left[\right.$ become $^{\prime}(s, e) \wedge$

$\overline{\left.\left.\left.\operatorname{have}^{\prime}\left(\operatorname{th}^{\prime}\left(\lambda s\left[R^{\prime}(\mathbf{b}, \mathbf{j}, s)\right]\right), r g^{\prime}\left(\lambda s\left[R^{\prime}(\mathbf{b}, \mathbf{j}, s)\right]\right), s\right)\right]\right]\right]}$

$=\exists v\left[\right.$ effector $^{\prime}(\mathbf{m}, v) \wedge \diamond \exists e\left[\operatorname{cause}^{\prime}(v, e) \wedge \exists s\left[\right.\right.$ become $\left.\left.^{\prime}(s, e) \wedge R^{\prime}(\mathbf{b}, \mathbf{j}, s)\right]\right] \wedge$

$\left.\operatorname{giving}^{\prime}(v) \wedge \exists e\left[\operatorname{cause}^{\prime}(v, e) \wedge \exists s\left[\operatorname{become}^{\prime}(s, e) \wedge \operatorname{have}^{\prime}(\mathbf{b}, \mathbf{j}, s)\right]\right]\right]$

$=\exists v\left[\right.$ effector $^{\prime}(\mathbf{m}, v) \wedge$ giving $^{\prime}(v) \wedge \exists e\left[\operatorname{cause}^{\prime}(v, e) \wedge \exists s\left[\operatorname{become}^{\prime}(s, e) \wedge\right.\right.$

$\left.\left.\left.\operatorname{have}^{\prime}(\mathbf{b}, \mathbf{j}, s)\right]\right]\right]$

$(=(44))$ 
Thus the aspect of root sensitivity whereby for some roots in ditransitive constructions the two variants of the dative alternation are identical in meaning is captured here, since the meaning of $\sqrt{\text { GIVE }}$ wholly subsumes the results entailed by both templates, nullifying the semantic difference between those templates. Crucially, the result entailed by the root is also furthermore sufficient to define the event's endpoint, whereby any event described by a $\sqrt{\text { GIVE }}$ predicate must end with possession of the theme by the recipient and no non-final subevent of this

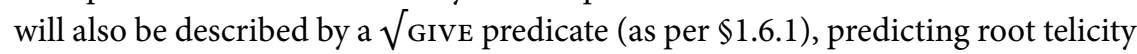
as well. In other words, the predicate is telic and the telicity here comes from the contribution of the root.

A second subtype of true caused possession roots are what we call "transfer of possession" roots (e.g. pay, serve, loan), which entail the same result as give (actual receiving) as in $(46 \mathrm{a}, \mathrm{b})$ but also additionally (temporary) loss of the theme by the agent, as in $(46 \mathrm{c}, \mathrm{d})$ (plus a different manner of course). ${ }^{17}$

(46) a. Mary paid John $\$ 5$, \#but he never possessed the money.

b. Mary paid $\$ 5$ to John, \#but he never possessed the money.

c. Mary paid John $\$ 5$, \#but she never ceased to possess the money.

d. Mary paid $\$ 5$ to John, \#but she never ceased to possess the money.

The combination of loss of possession by the causer and coming to possess by the recipient defines transfer of possession. To analyze this, we propose the the denotation for the root of pay given in (47a), which relies on placing a condition on the initial possessional state of the theme in the change-of-state event $e$, which ensures that transfer of possession arises for a scale that technically just represented coming to possess as per $\$ 3.5$ (where for any change event $e$ we define init' $(e)$ as the initial state $s^{\prime}$ for $e$; see $\$ 1.6 .1 .3$ ). This root will in turn derive (47b) as the final meaning of the initial clauses in (46) (here and below we omit the steps of substitution of logically equivalent terms, which will proceed analogously to (45)): ${ }^{18}$

\footnotetext{
${ }^{17}$ Beavers (2011a: 27-8) illustrates this root class with sell, but as a reviewer points out it is possible to sell somebody something without losing it. For example, in Mary sold fishing rights to John on at least one interpretation Mary need not lose her own fishing rights if she has them. Thus the degree to which Mary sold John a book does entail loss of the book by Mary it is an artifact of possession of books. To our intuitions, though, pay does not have this property. The act of payment is a definitional transfer of ownership.

${ }^{18}$ That the recipient does not possess the theme prior to the final state follows from the nature of possessional scales as in $\$ 3.5$. Conversely, the entailment of loss is explicitly stated here in terms of the agent first having the theme and then not having it since this does not follow from anything about possession scales. Note that loss or leaving type semantics, i.e. a "from" type meaning, has been independently claimed to be a templatic meaning as per Pylkkänen (2008: 49-64), suggesting another way in which such roots violate the Bifurcation Thesis.
} 
a. $\llbracket \sqrt{\mathrm{PAY}} \rrbracket=\lambda P \lambda x \lambda v\left[\right.$ paying $^{\prime}(v) \wedge \exists e\left[\operatorname{cause}^{\prime}(v, e) \wedge \exists s\left[\operatorname{become}^{\prime}(s, e) \wedge\right.\right.$ $\operatorname{have}^{\prime}\left(\operatorname{th}^{\prime}(P), x\right.$, init' $\left.^{\prime}(e)\right) \wedge \neg \operatorname{have}^{\prime}\left(\operatorname{th}^{\prime}(P), x, s\right) \wedge$ $\left.\left.\left.\operatorname{have}^{\prime}\left(\operatorname{th}^{\prime}(P), r g^{\prime}(P), s\right)\right]\right]\right]$

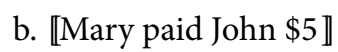
$=\exists v\left[\right.$ effector $^{\prime}(\mathbf{m}, v) \wedge$ paying $^{\prime}(v) \wedge \exists e\left[\operatorname{cause}^{\prime}(v, e) \wedge \exists s\left[\right.\right.$ become $^{\prime}(s, e) \wedge$ $\operatorname{have}^{\prime}\left(\$ \mathbf{5}^{\prime}, \mathbf{m}\right.$, init $\left.\left.\left.\left.{ }^{\prime}(e)\right) \wedge \neg \operatorname{have}^{\prime}\left(\$ \mathbf{5}^{\prime}, \mathbf{m}, s\right) \wedge \operatorname{have}^{\prime}\left(\$ \mathbf{5}^{\prime}, \mathbf{j}, s\right)\right]\right]\right]$

This root ensures telicity for the same reason give does, and again there is no predicted contrast in the dative alternation-just as with give the entailed result of receiving that comes from the root subsumes the results of both templates, obliterating the distinction between them in this instance of the dative alternation.

Finally, a subtype of this subclass includes what we call "transfer of possession by motion" roots (e.g. hand, pass), which entail the same as transfer of possession roots plus that physical motion of the theme from the initial to the final possessor also occur, as in (48), thus requiring change along a possession by motion scale:

(48) a. Mary handed John the book, \#but it never came to be on his person.

b. Mary handed the book to John, \#but it never came to be on his person.

c. Mary handed John the book, \#but it never left her person.

d. Mary handed the book to John, \#but it never left her person.

The hand root in (49a) (from which leaving follows by the nature of path scales and possession by motion scales as in $\$ 3.5$ ) derives (49b) for the initial clauses of (48):

a. $\llbracket \sqrt{\text { HAND } \rrbracket}=\lambda P \lambda x \lambda v\left[\operatorname{handing}^{\prime}(v) \wedge \exists e\left[\operatorname{cause}^{\prime}(v, e) \wedge \exists s\left[\right.\right.\right.$ become $^{\prime}(s, e) \wedge$ $\operatorname{have}^{\prime}\left(\operatorname{th}^{\prime}(P), x\right.$, init' $\overline{(e)) \wedge a t^{\prime}\left(\operatorname{th}^{\prime}(P), x, \text { init }^{\prime}(e)\right) \wedge \neg \operatorname{have}^{\prime}\left(t^{\prime}(P), x, s\right) \wedge}$ $\left.\left.\left.\operatorname{have}^{\prime}\left(t^{\prime}(P), r g^{\prime}(P), s\right) \wedge a t^{\prime}\left(t h^{\prime}(P), r g^{\prime}(P), s\right)\right]\right]\right]$

b. $\exists v\left[\right.$ effector $^{\prime}(\mathbf{m}, v) \wedge$ handing $^{\prime}(v) \wedge \exists e\left[\operatorname{cause}^{\prime}(v, e) \wedge \exists s\left[\right.\right.$ become $^{\prime}(s, e) \wedge$ $\operatorname{have}^{\prime}\left(\mathbf{b}, \mathbf{m}\right.$, init $\left.^{\prime}(e)\right) \wedge \operatorname{at}^{\prime}\left(\mathbf{b}, \mathbf{m}, \operatorname{init}^{\prime}(e)\right) \wedge \neg \operatorname{have}^{\prime}(\mathbf{b}, \mathbf{m}, s) \wedge$ $\left.\left.\left.\operatorname{have}^{\prime}(\mathbf{b}, \mathbf{j}, s) \wedge a t^{\prime}(\mathbf{b}, \mathbf{j}, s)\right]\right]\right]$

Just as with pure caused possession and non-motion transfer of possession roots, an actual result obtains and we have a strengthening over both templates. Thus the roots discussed in this section all have in common (a) entailing a non-prospective result, namely actual having, that is sufficient to ensure telicity and (b) subsuming the meaning of both templates, meaning no contrast arises in the dative alternation for any root here, explaining root sensitivity. In all cases the root is what truth conditionally contributes the relevant results, meaning the root has as part of its 
lexical entailments notions of change of state, co-location, and possession, all of which are otherwise templatic notions, something that should not be possible under Bifurcation.

However, there is a potential counterargument to this claim. In particular, the possession relation-sufficient alone for telicity-is simply a further refinement of the templatic result state. Thus arguably the root is doing nothing more than describing the final state that is introduced already by the template, and somehow ensuring that it obtains at the evaluation world $w$. Thus the notions of change and possibly also possession itself could arguably be coming from the template, not the root, consistent with Bifurcation. However, there are problems with this alternative understanding of the relative contribution of the root and the template. First, while it is true that the indirect object template already entails prospective caused coming to possess, with the to template the possession relation must be coming from the root since possession is not obligatory for to templates. This alone is inconsistent with Bifurcation. Furthermore, while the semantics of change is part of the meaning of both templates, there are separate changes-of-state arising from the use of these particular roots that are orthogonal to the (prospective) changes that the templates entail, namely the loss, leaving, and arriving, that we claim individual roots introduce and which involve separate conditions of the templatic notions of possession and location. Still further, no actual change of state is entailed in either case. It is simply a possibility in both templates. For the root to ensure that change actually occurs without introducing change itself it would have to somehow access the modal operator and restrict its modal base to just $w$, a complex (though not impossible) semantics. On our analysis actual change straightforwardly follows from the root conjunctively bringing to bear its own change of state using the same basic lexical semantic primitives templates use in a way that naturally subsumes what is introduced by the templates. Thus possession, co-location, and even change seem to be part of root meanings. Not all of what the complete event structures entail is wholly parasitic on what is entailed by the template alone. The overlaying of orthogonal actual changes is even more striking in the next class of roots we discuss, where such relations actually emerge as relevant for telicity in the absence of actual receiving.

\subsubsection{Release roots}

We now turn our attention to a class of roots that lack some of the results that defined the previous class, yet nonetheless still entail results sufficient to ensure telicity. In particular, ballistic motion roots (e.g. throw, toss, granny ${ }^{19}$ ) and sending

${ }^{19}$ In the dialect of the first author of this book, Coach Jester grannied the ball (into the net) means Coach Jester released the ball using an underhand motion from between his legs. In other dialects this might be Coach Jester took a granny shot (Jacques Lafleur, p.c.). 
roots (e.g. send) differ from those above in entailing neither possession nor arrival. Rather, both of these notions are prospective:

(50) a. John sent/lobbed Mary the ball, but the heavy downpour stopped her getting it.

b. John sent/lobbed the ball to Mary, but the heavy downpour stopped her getting it.

c. John sent/lobbed Mary the ball, but the heavy downpour stopped it arriving.

d. John sent/lobbed the ball to Mary, but the heavy downpour stopped it arriving.

So why are these predicates telic? Following Beavers (2011a: 31-7) the relevant result is that the agent loses the theme and/or the theme leaves the agent, something non-cancelable in both variants:

(51) a. John sent/tossed Mary the ball, \#but he never gave it up.

b. John sent/tossed the ball to Mary, \#but he never gave it up.

c. John sent/tossed Mary the ball, \#but it never left.

d. John sent/tossed the ball to Mary, \#but it never left.

Loss or leaving is sufficient to ensure telicity: the final point of the event as described by the predicate is reached when the causer and the theme are no longer in a possessional or co-location relationship of a particular sort, and no subevent lacking such a transition away from such a state is also described by the same predicate. Crucially, this meaning is not coming from the templates since it does not exist in all root plus template combinations for either template (e.g. as discussed above and again below, bequeath in either construction lacks any such entailments). Thus loss or leaving must be coming from this particular class of roots, which we refer to as "release" roots. Furthermore, these roots also arguably entail prospective co-location due to the ballisticness of the manner for ballistic motion verbs and the nature of sending, for which it is assumed there is an expected recipient or goal, thus subsuming the result of the to construction.

Putting the pieces together, something like (52) in the to template will have the following meaning, treating the relevant result as not being at the agent, defined as the theme being at some distinct location at the end of the event, from which at least some type of loss of possession follows. (Here and below we just give final meanings without giving the root meanings separately, the important aspects of which can be deduced from what is underlined.) 
(52) John threw the ball to Mary.

$\exists v\left[\right.$ effector $^{\prime}(\mathbf{j}, v) \wedge$ throwing $^{\prime}(v) \wedge \exists e\left[\operatorname{cause}^{\prime}(v, e) \wedge \exists s\left[\right.\right.$ become $^{\prime}(s, e) \wedge$
$\operatorname{at}^{\prime}\left(\mathbf{b}, \mathbf{j}\right.$, init $\left.^{\prime}(e)\right) \wedge \overline{\left.\left.\exists z\left[a t^{\prime}(\mathbf{b}, z, s) \wedge z \neq x\right]\right]\right] \wedge \diamond \exists e\left[\operatorname{cause}^{\prime}(v, e) \wedge\right.}$
$\underline{\left.\left.\exists s\left[\text { become }^{\prime}(s, e) \wedge a t^{\prime}(\mathbf{b}, \mathbf{m}, s)\right]\right]\right]}$

The root-entailed prospective result-prospective co-location-subsumes the more general prospective $R^{\prime}$ relation of the to construction. However, crucially, the prospective indirect object result, namely prospective receiving, is not entailed by the root, evidenced again by London Office effects:

(53) a. John threw Mary/\#London the ball.

b. John threw the ball to Mary/London.

Thus this contribution of the indirect object construction will augment what is coming from the root, which means that the two variants of the dative alternation with such roots will mean different things, i.e. (52) and (54) will differ only in the presence of prospective receiving in the latter:

(54) John threw Mary the ball.

$\exists v\left[\right.$ effector $^{\prime}(\mathbf{j}, v) \wedge \diamond \exists e\left[\operatorname{cause}^{\prime}(v, e) \wedge \exists s\left[\operatorname{become}^{\prime}(s, e) \wedge \operatorname{have}^{\prime}(\mathbf{b}, \mathbf{m}, s)\right]\right] \wedge$ throwing $^{\prime}(v) \wedge \exists e\left[\right.$ cause $^{\prime}(v, e) \wedge \exists s\left[\right.$ become $^{\prime}(s, e) \wedge a t^{\prime}\left(\mathbf{b}, \mathbf{j}\right.$, init $\left.^{\prime}(e)\right) \wedge$

$\left.\left.\exists z\left[a t^{\prime}(\mathbf{b}, z, s) \wedge z \neq x\right]\right]\right] \wedge \diamond \exists e\left[\operatorname{cause}^{\prime}(v, e) \wedge \exists s\left[\right.\right.$ become $^{\prime}(s, e) \wedge$ $\left.\left.\left.a t^{\prime}(\mathbf{b}, \mathbf{m}, s)\right]\right]\right]$

This again explains root sensitivity. Root telicity in principle should follow from the root's entailed co-location state, though here another factor is relevant. All the root says in (52) and (54) is that the theme is not with the agent anymore. But since this could be compatible with a range of following locations (e.g. a foot away, two feet away, three feet, in linear order) then in principle the predicate could be atelic since some such events are non-final subevents of others. However, as we discuss in $\$ 3.7$ ballistic motion verbs are (with one possible exception) punctual, suggesting that the scale here is constrained somehow to be two part, corresponding to the theme being with the agent and then not with the agent, likely due to the specific manner involved (i.e. throwing and tossing definitionally culminate when the release occurs). Assuming this to be an additional condition on (most) ballistic motion verbs (e.g. derived somehow from the manner, though we leave the details out) then telicity will follow. Interestingly, the relevant result state is not an augmentation of what the template entails. It is a wholly distinct 
state, suggesting that roots can contribute states relevant for telicity not contingent upon the actual prospective result of the template they occur in.

\subsubsection{Arrival roots}

Another broad class of roots consists of roots of accompanied motion (e.g. bring, take) and continuous application of force (e.g. carry, haul, drag), which lexically entail arrival, but crucially again not caused possession (we do not give indirect object variants for continuous application of force roots since these generally lack such variants, though see $\$ 3.8):{ }^{20}$

(55) a. Mary brought/hauled the dossier to the council, but she never gave it up.

b. Mary brought the council the dossier, but she never gave it up.

c. Mary brought/hauled the dossier to the council, \#but it never arrived.

d. Mary brought the council the dossier, \#but it never arrived.

Thus again there is a root-determined result sufficient to derive telicity, namely co-location, though again this result is orthogonal to what is entailed by the indirect object template at least, since that template only brings to bear prospective possession, something that the root does not entail. The root though does subsume the meaning of the to template, since actual co-location subsumes a prospective $R^{\prime}$ relation.

Putting the pieces together, the meanings for the two variants of the dative alternation will resemble something like the following, where the theme ends up at the goal described by the indirect object or theme (assuming the agent's own change of location follows from this plus the particular manner) and where there is again a contrast across the dative alternation since the indirect object template augments the meaning with prospective receiving, predicting a London Office effect in that variant but not the to variant (e.g. Mary brought the dossier to London vs. \#Mary brought London the dossier):

a. Mary brought the dossier to the council.

$$
\begin{aligned}
& \exists v\left[\text { effector } ^ { \prime } ( \mathbf { m } , v ) \wedge \text { bringing } ^ { \prime } ( v ) \wedge \exists e \left[\operatorname { c a u s e } ^ { \prime } ( v , e ) \wedge \exists s \left[\operatorname{become}^{\prime}(s, e) \wedge\right.\right.\right. \\
& \left.\left.\left.a^{\prime}(\mathbf{d}, \mathbf{c}, s)\right]\right]\right]
\end{aligned}
$$

\footnotetext{
${ }^{20}$ These roots have another non-indirect object use without the to PP that does not entail arrival at some particular place or any ultimate clear change of location as in John carried the books (around). However, as Beavers and Koontz-Garboden (2017a) discuss in detail, with some motion verbs circular motion is possible, wherein there is a complete scalar change qua change of location along a spatial scale, but it is an artifact of the potential for circularity in spatial scales that the final state is the same as the initial state. That the final state is left unspecified does not mean arrival does not occur. It just means that arrival at some specific location is not entailed (see $\$ 1.6 .1 .1$ ). Thus a use without a to PP is akin to a degree achievement on an atelic interpretation.
} 
b. Mary brought the council the dossier.

$$
\begin{aligned}
& \exists v\left[\operatorname{effector}^{\prime}(\mathbf{m}, v) \wedge \diamond \exists e\left[\operatorname{cause}^{\prime}(v, e) \wedge \exists s\left[\operatorname{become}^{\prime}(s, e) \wedge \operatorname{have}^{\prime}(\mathbf{d}, \mathbf{c}, s)\right]\right] \wedge\right. \\
& \underline{\text { bringing } \left.^{\prime}(v) \wedge \exists e\left[\operatorname{cause}^{\prime}(v, e) \wedge \exists s\left[\operatorname{become}^{\prime}(s, e) \wedge a t^{\prime}(\mathbf{d}, \mathbf{c}, s)\right]\right]\right]}
\end{aligned}
$$

Once again, the root entails notions of change and co-location that traditionally are viewed of as templatic notions.

\subsubsection{Electronic release and arrival roots}

In this section we briefly mention one class of roots that we have not discussed in detail yet, namely verbs of instrument of communication qua electronic means of communication such as email, fax, scp (Secure Shell Copy, a method of securely transferring files across computers in primarily UNIX-like systems), and ftp (File Transfer Protocol, a popular means of transferring files across platforms). These roots differ from all other ditransitive roots in that it is not technically the theme itself which undergoes prospective change of location or coming to be possessed, but rather an electronic copy of the theme. In this case it could thus be that both the causer and the recipient have a copy of the theme at the end of the event:

I ftp'd/faxed the dossier to Bill so we would both have a copy.

That said, actual possession of the theme is not required in either variant of the dative alternation for any of these predicates:

(58) a. I faxed/emailed/scp'd the file to Mary, but she never received it.

b. I faxed/emailed/scp'd Mary the file, but she never received it.

However, these roots do seem to split in terms of whether they fundamentally entail only release like send or throw or arrival like haul or bring (Pesetsky 1995: 143). In particular, email seems to pattern like an electronic version of send or mail, whereas $f t p$ or $s c p$ seem to pattern like electronic versions of bring:

(59) a. Kim emailed the file to Mary, but it never reached her inbox/\#the copy never left.

b. Kim ftp'd the file to Mary, \#but it never reached her server.

This distinction may follow from the types of specific manners involved here. Emailing is viewed as complete when the email sends, but like sending or postal mailing this does not ensure arrival, whereas transferring operations using protocols such as ftp or scp typically involve an extended process of byte-by-byte transfer that are monitored until fully complete, at which point the process ends 
(Pesetsky 1995: 143 and Krifka 2004: 22-3 make similar points). Alternatively, a reviewer asks if the difference between email and ftp could instead be due to the different sorts of actions one takes prior to the transfer. With email one deals just with one's email client but with ftp there are a variety of steps including logging into a remote server, which would take more inherent steps and thus arguably ensure a more durative event. This, however, depends on there being a specific set of subactions associated with each type of action, whereas in reality there are numerous ways to email and ftp that involve more or fewer steps (e.g. emailing via remote login would involve contacting another server, whereas ftping by a single command line command could be deemed a single step). The only constant in each process is the single send function for email vs. the byte-by-byte transfer for $f t p$, suggesting this is the preferable analysis. These two subtypes of electronic transfer verbs are therefore subsumed under the two previous classes, and we do not discuss them any further here and assume that they can be given analogous analyses, albeit with the addition of some notion of creating a copy of the theme. (See Beavers 2011a: 38-41 for further discussion of some of the unique properties of this subclass of ditransitive verbs.)

\subsubsection{Future having roots}

The final root class are "future having" roots (e.g. bequeath, leave, allot). These are more complex than any of the roots discussed above, since loss, leaving, arrival, and receiving are all cancelable:

(60) a. John bequeathed/left Bill his fortune...

i. ... but Bill died before he could take it.

(no possession)

ii. ... but John rescinded the will before he died and kept the money.

(no loss)

iii.... and the money stayed in the same account even after Bill inherited it.

(no leaving/arrival)

b. John bequeathed/left his fortune to Bill...

i. ... but Bill died before he could take it.

(no possession)

ii. ... but John rescinded the will before he died and kept the money.

(no loss)

iii.... and the money stayed in the same account even after Bill inherited it.

(no leaving/arrival)

Prospective possession is entailed, however, i.e. the London Office effect obtains in both variants: 
(61) a. \#John bequeathed/left London his fortune.

b. \#John bequeathed/left his fortune to London.

So why would these predicates be telic? There is a intuitive reason: they are all performative in the sense that they entail the creation of a contract or other obligation meant to ensure possession at a future date, though the temporal distance between the creation of the obligation and the actual potential receiving leaves open the possibility that the obligation could be nullified or otherwise stopped from occurring (e.g. if the causer happens to spend all of the money). Creation of an obligation is a (presumably punctual) transition (from no obligation in existence to an obligation in existence), and this is sufficient to ensure telicity as with any other creation or performative predicate. (See Beavers $2011 \mathrm{~b}$ on nonperformative creation verbs as verbs of scalar change.) The nature of the obligation furthermore ensures that these roots entail prospective possession, subsuming the results of both the indirect object and to templates, meaning there is no contrast in the alternation. This explains the root sensitivity fact that the London Office effect obtains in both variants and the two variants are otherwise generally paraphrases.

It is not our goal to give a theory of performativity or the relevant type of performative creation here, so we do not give formal representations, though it should be clear how representations analogous to those above would capture the facts. In this case the result that derives telicity - creation of an obligation-is truly distinct from the results of either of the templates, more so than for any of the root-supplied changes discussed above, which in many cases involved the same ingredients (e.g. motion and possession) that are also found in the template. These verbs thus provide some of the strongest evidence that roots can determine telicity via changes of state in ways wholly independent of the templates they occur in.

\subsubsection{Summary}

We have seen that the following basic root-supplied change of state can determine root telicity and root sensitivity alone or in combination:

(62) a. Coming to possess - the recipient comes to possess the theme

b. Loss of possession - the causer ceases to possess the theme

c. Leaving - the theme leaves a specified source

d. Arrival - the theme arrives at a specified goal

e. Creation of obligation - an obligation comes to exist by which the theme must undergo transfer of possession from the causer to the recipient at some point in the future 
All of the roots in (6) and (7) entail at least one of these results, thus defining them by a (cross-classification) of these entailments. But the templates themselveswhile compatible with and sometimes entailed by them-do not entail these notions. The roots do. This in turn already derives a root typology that cashes out in a series of semantic verb classes in terms of how the variants of the dative alternation are interpreted that is orthogonal to the templates (i.e. "root sensitivity" yields "verb sensitivity"). We turn to further evidence for the roots having these properties next, some of which will also show that that the root typology we suggest here also yields particular argument realizational effects in its interaction with the indirect object and to templates. This justifies that the roots we discuss here have grammatical consequences just as result roots in the previous chapter yielded not just distinct semantic verb classes but classes that have direct morphological consequences as per $\$ 2.4 .1$.

\subsection{Further evidence for change of state in roots: Root durativity}

In this section we take a brief interlude from arguing that roots entail templatic meaning to discuss a piece of supporting evidence that the roots of ditransitives, despite being manner roots, contribute information about the scalar change of the entire predicate, namely the ways these roots figure into the durativity of the overall predicate. Recapping $\$ 1.6 .1 .1$, we follow Beavers $(2008,2012)$ in defining durativity in terms of event complexity-punctual events are two-point events that have just a beginning and an end, whereas durative events are more complex events that have additional subevents as well as a beginning and an end. A standard diagnostic for the durativity of telic predicates (Kearns 2000: 206) is the interpretation of in an hour modifiers: with punctual predicates the interpretation is after an hour, whereas with durative predicates the interpretation is after an hour or during an hour. For verbs describing scalar change durativity in turn correlates with the complexity of the scale, where punctual predicates tend to describe changes over non-gradable (two point) scales, while durative predicates tend to describe changes over gradable scales (greater than two points). The correlation of event and scalar complexity follows from the homomorphic mapping relation between parts of the event and parts of the scalar structure, where (intuitively) the more change there is along a scale the more event there must be for that change to occur, and thus more complex scales yield durative events. A simple transition along a non-gradable scale from one state to another takes just two points in time, and thus correlates with a punctual event. ${ }^{21}$

${ }^{21}$ As discussed in \$1.6.1.1, the mereological complexity of the theme can also have an effect on durativity, whereby theme DPs that refer to mereologically complex objects may determine durative readings on the interpretation that different parts of the theme undergo the change entailed by the predicate at different times over the course of the event. Similarly, complex manners can also yield 
The question we address here is the contribution of the root to the durativity of the predicate in ditransitives. Whether durativity and/or scalar complexity is considered templatic in and of itself is debatable. If languages have functional "durativity markers" then presumably it is. For example, Karitiana (an Arikén language spoken in the northwest of Brazil) has overt morphological processes (namely reduplication) used to signal that multiple events occurred (Müller and Sanchez-Mendes 2008), achieving a durativity effect whereby inherently punctual predicates as in (63a) that cannot be iterated are unacceptable with pluractional morphology as in (63b), but iterable predicates as in (63c) are acceptable with pluractional morphology as in (63d):

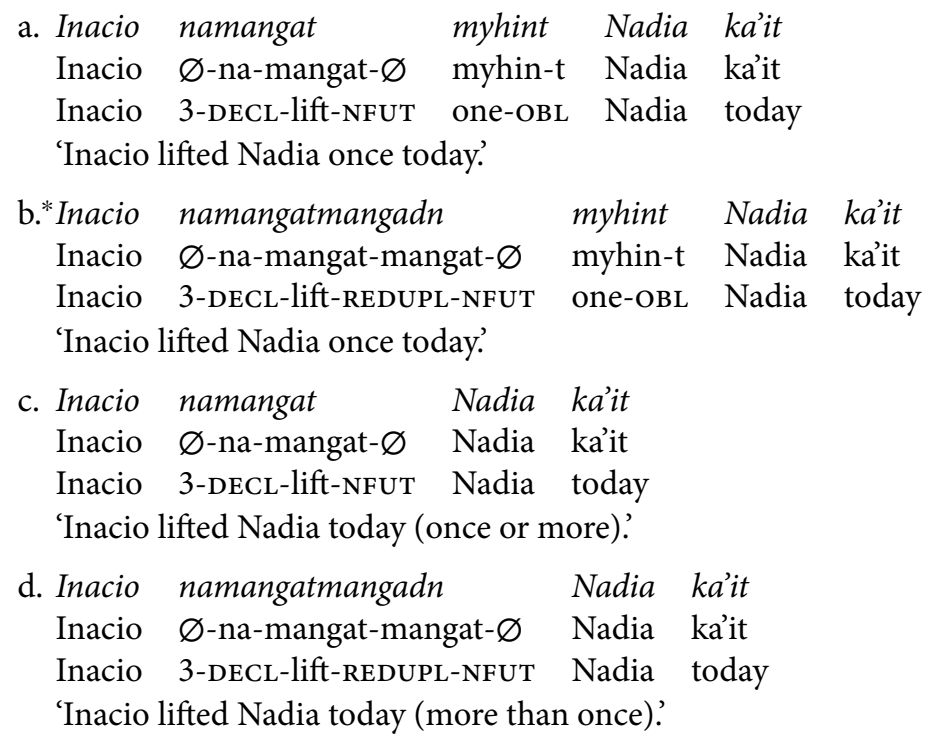

(Müller and Sanchez-Mendes 2008: 450-1, (30)-(33))

The existence of regular morphological processes that derive durativity might be taken as evidence that durativity itself ought to be treated as templatic.

But even if durativity and scalar complexity are not templatic notions, if the roots of ditransitives entail changes of state qua scalar change then we might expect that they should impose other conditions on the change such as scalar complexity constraints giving rise to root-conditioned durativity. In fact, such a finding was already made in Chapter 2 in relation to the observation that roots

durativity, as discussed in $\$ 1.6 .2 .2$. Our interest here is primarily in durativity as determined by scales and as such we will use DP theme arguments whose referents are naturally interpreted as atomic and thus changing all at once. As for manner, as it turns out many ditransitives are punctual, suggesting that the manners are not themselves complex; for more on the interplay between manners and changes in durativity see $\$ 4.3 .3$. 
specify for temporal and atemporal change (see $\$ 2.2 .4$ and discussion therein). In that chapter we implemented this for simplicity's sake as a constraint on the type of event described, but fundamentally this determines a constraint on the entity (e.g. time or space) over which the change occurs, and thus placing constraints directly on the relata of a become $e^{\prime}$ relation. A similar finding in the domain of ditransitives that roots impose constraints on the nature of the scale-another entity involved in a become $e^{\prime}$-type relation as per $\$ 1.6 .1 .3$ - would lend further and similar support to the conclusion of the preceding sections that the roots of ditransitive verbs of caused possession in general figure into the aspectual properties of the overall predicate by defining aspects of the change of state that is part of its meaning.

First and foremost, verbs of giving are generally all punctual (a point made also by Jackendoff 1996: 330 and Krifka 2004: 6):

(64) a. Mary can give Leah a book in ten minutes.

(after ten minutes)

b. Mary can give a book to Leah in ten minutes.

(after ten minutes)

c. Mary can hand Leah the salt in five seconds.

(after five seconds)

d. Mary can hand the salt to Leah in five seconds.

(after five seconds)

This follows for verbs of giving if these roots entail solely caused possession, analyzable as in $\$ 3.5$ as a transition from a state at which the recipient does not possess the theme to a state of the recipient possessing it on a non-gradable scale (see also Jackendoff 1996: 330). Arguably, though, this is derivable independent of the root-the indirect object template already defines (prospective) possession, and so the non-gradability of the scale could follow from that. However, this would not explain why these facts necessarily carry over to the to template. Here the template-defined relation $R^{\prime}$ could resolve to either caused possession or caused co-location, or both simultaneously. Yet in general neither motion nor possession by motion are necessarily or by default non-gradable changes. Thus it seems that the endpoints defined by individual roots are significant in determining the scalar gradability of the entire predicate. Furthermore, hand is a special case, since it also entails motion, but its specific manner (a transition from one hand to another in close proximity) ensures a short physical path, which as discussed in $\$ 3.5$ figures into the definition of the scale. Thus (65a), involving little to no distance between the causer and recipient, is acceptable, but (65b), where the context clarifies that the causer and recipient are quite far apart, is infelicitous:

(65) a. Without leaving her seat, Kim handed the person sitting next to her a quarter.

b. \#Without leaving her seat, Kim handed the person on the other side of the room a quarter. 
This constraint is thus clearly coming from the root and not the template, providing additional evidence that the root is somehow contributing information that ensures scalar non-gradability of the entire predicate since they are defining scalar properties of the change despite technically being manner roots. ${ }^{22}$

Similarly, bequeath and other future having verbs are like giving verbs in deriving punctual predicates, save that the transition is not from not possessing to possessing but from the coming about of a contractual obligation through which the recipient goes from not being the prospective possessor to being the prospective possessor, another binary contrast:

(66) a. Chris can bequeath Leah a gem in ten minutes.

(after ten minutes)

b. Chris can bequeath a gem to Leah in ten minutes.

(after ten minutes)

Here again this must be coming from the root and not the template. Thus the particular result states contributed by these roots define non-gradable scales, further supporting evidence that the roots contribute change-of-state information.

Another interesting case are sending and ballistic motion verbs, which are also mostly punctual:

a. Mary can throw a ball to John in five seconds.

b. Mary can throw John a ball in five seconds.

c. Mary can sling a ball to John in five seconds.

d. Mary can sling John a ball in five seconds.

e. Mary can toss a ball to John in five seconds.

f. Mary can toss John a ball in five seconds.

g. Mary can send a ball to John in five seconds.

h. Mary can send John a ball in five seconds. (after five seconds) (after five seconds) (after five seconds) (after five seconds) (after five seconds) (after five seconds) (after five seconds) (after five seconds)

Here there is no specific new having (or co-location) that defines the result state, but rather a loss and/or leaving outcome that does. But in this case the same kind of reasoning as above applies: the causer goes from having the theme to not having it via some presumably very short action along a short path, and this is a binary contrast that gives rise to a non-gradable scale. Furthermore, in this case the non-gradable change is in addition to any prospective outcome entailed by either template, meaning that the relevant constraints are indeed coming from the root and furthermore that these results and their aspectual effects are not somehow

\footnotetext{
${ }^{22}$ A reviewer suggests that (65b) might be acceptable if Kim handed the quarter to the person next to her, who then handed it to the person next to them, and so on around the room until it reaches the person on the other side of the room. Our own judgments is that this reading is disallowed. For speakers who accept it, this just suggests that they have lexicalized a use that allows a durative process.
} 
incidental to the overall aspectual properties of the predicate but are in fact part and parcel of it.

Still further, there is a small class of ballistic motion verbs that do readily admit durative readings, including in particular ricochet, which can occur as a ditransitive verb, especially in sports contexts:

(68) Baker ricocheted the ball to Nicole Randall, who rifled the ball into the net to tie the match at 1-1. <http://www.hillcountrynews.com/stories/ whs-girls-soccer-heads-for-third-round,72349> (Accessed October 2017)

For some speakers, ricochet allows a natural durative reading, especially when context clarifies that the path be extended somehow:

(69) [ The walls of the court are very craggy and ricocheting takes time ]

Baker can ricochet the ball to Randall in 30 seconds.

(during/after 30 seconds)

The reason this has a durative reading is likely to do with the nature of its idiosyncratic manner of motion: this type of motion requires at least three spatial points - a place before impact, a place of impact, and a place after impact-and thus the very nature of the manner is defined in terms of motion along a gradable scale (i.e. an extended path), which is in turn a type of change. This is similar to the fact independently observed by Dowty (1979: 171) regarding the intransitive motion verb waltz, whose manner is such that there must necessarily be a threepoint path involved. The constraints on type of motion in ballistic motion verbs conspire with whatever constraints are in the template to produce the overall aspectual behavior of the entire predicate.

A final case study consists of accompanied motion roots with a specific manner, i.e. continuous application of force verbs, which are all durative (here again we give only the to variants since these verbs are typically not acceptable in the indirect object variant, though see $\$ 3.8$ for more on this):

(70) a. Mary can haul the very heavy boulder to John in five minutes.

(during/after five minutes)

b. Mary can carry the very heavy boulder to John in five minutes. (during/after five minutes)

c. Mary can drag the very heavy boulder to John in five minutes.

(during/after five minutes)

One might suggest that this has to do with the particular manner involved: hauling and dragging in particular might be manners that (once one spells out the explicit details of the type of friction involved) cannot be defined without reference to at 
least several points along the path. However, interestingly, accompanied motion verbs that lack manners, namely the two deictic verbs bring and take, are also durative despite the fact that they have no particular manner in the sense that drag and haul do, i.e. while they idiosyncratically define some deixis information they describe no particular action:

(71) a. I can bring/take Mary the letter in five minutes.

(during/after five minutes)

b. I can bring/take the letter to Mary in five minutes.

(during/after five minutes)

These two roots somehow require a greater proximity between the causer and the recipient, the converse of hand above:

(72) [ Mary and John are standing side-by-side ]

* Mary brought/took John the letter.

The fact that these are durative suggests that the roots are constraining scale and/or path length, since the indirect object and to templates do not themselves determine path length. In this case though really it seems to just be a direct constraint on the scale of the overall predicate-it is not obvious that any constraints on manner or the type of motion would otherwise derive this, providing some of the clearest evidence that the roots of ditransitives have access to and directly constrain the scale that measures out the change described by the overall predicate, much in the way we observed in Chapter 2 that the roots of change-of-state verbs also have access to and directly constrain the nature of the change event tied to those roots to be exclusively temporal or not.

Taken all together, what these data suggest is that the durativity of ditransitive verbs is not conditioned exclusively by the template, but rather is conditioned by the root, exactly as expected if they entail change of state qua scalar change more generally. These observations thus serve as suggestive supporting evidence that the root contributes significant, possibly templatic, semantic information regarding the nature of the change of state. Roots furthermore do so despite sitting in a manner position in the event structure. Of course, these facts are in principle also compatible with an approach that assumes Bifurcation and thus that these roots are purely manner-entailing. It could be that somehow from the manner adjoined position the roots come with semantic constraints that pick out the relevant scale of change and impose complexity constraints on it, which again are not necessarily templatic in and of themselves, without also entailing wholesale changes of state. The selectional conditions required to make such an analysis work would perhaps be unexpected and would raise the question of why the roots would be imposing scalar complexity constraints over scales that they do not introduce or otherwise 
impose constraints on themselves. Thus an analysis that assumes that change of state can come directly from the root is more naturally compatible with the root durativity facts discussed here.

\subsection{The Dative Alternation: Root-determined argument realization}

A final piece of evidence that roots entail templatic information, in this case possession again, comes from the fact that not all ditransitive roots regularly show the dative alternation. In particular, continuous application of force roots disprefer indirect object constructions, as illustrated in (73):

(73) a. John carried/hauled/dragged/pushed the ball to Sandy.

b. ??John carried/hauled/dragged/pushed Sandy the ball.

Krifka (2004: 17-9) suggests that manner of motion requires the construction to entail motion, which he claims the indirect object template does not do, and thus these roots will not occur in such a template. However, indirect objects do not actually exclude motion per se, and in fact some roots such as hand require it in both templates (see \$3.3.1). So this cannot explain (73).

However, the ban against these roots occurring with indirect objects is not in fact categorical, but is rather more of a tendency. In particular, Bresnan and Nikitina (2009) give numerous examples of acceptable, naturally attested cases of continuous application of force roots in the indirect object template (these are from Bresnan and Nikitina 2009: 164, (11)):

(74) a. Karen spoke with Gretchen about the procedure for registering a complaint, and hand-carried her a form, but Gretchen never completed it.

b. As Player A pushed him the chips, all hell broke loose at the table.

c. Nothing like heartburn food. "I have the tums." Nick joked. He pulled himself a steaming piece of the pie. "Thanks for being here."

d. "Well ... it started like this ..." Shinbo explained while Sumomo dragged him a can of beer and opened it for him, "We were having dinner together and..."

e. Therefore, when he got to purgatory, Buddha lowered him the silver thread of a spider as his last chance for salvation.

So the real question here is why such roots disprefer the indirect object template, if not wholly disallow it. Bresnan and Nikitina (2009) themselves largely pin this on idiosyncratic lexicalization, positing a highly ranked yet violable constraint in their Optimality Theoretic analysis of the dative alternation that disprefers the 
occurrence of these particular verbs in the indirect object template unless some reranking occurs to make constraints relating word order to information structure or heaviness more prominent so that the indirect object template preferred.

Beavers (2011a: 44-8) argues that these facts instead follow from two factors having to do with clashes between different types of possession as discussed in $\$ 3.2$, all of which ultimately point to possession being a root entailment. First, due to the particular manners involved with these roots-whereby the causer is constantly applying force to the theme-the causer must have the theme in the sense of controlling it at every point in the event (e.g. He has my car (for the weekend)), a subtype of possession identified by Tham (2006: 138-9) that for convenience we refer to as have ${ }_{C}^{\prime}$. But the indirect object construction requires the recipient to also prospectively have the theme at the last point in the event. This could in principle be any type of possession, though the most prototypically would be alienable possessional, which we refer to as have ${ }_{p}^{\prime}$. Crucially, putting these two conditions together, at the very last point in the event the causer would have ${ }_{C}^{\prime}$ the theme (owing to the manner) and the recipient would (prospectively) have ${ }_{P}^{\prime}$ it, i.e. the theme has two separate possessors of potentially two different sorts. This is not contradictory per se, since in He has my car (for the weekend) this is exactly the state of affairs that obtains in the sense that throughout the controlling situation someone else is the alienable possessor of the car.

But English possessive constructions independently seem to disprefer a situation where there is a change in alienable possession of the theme while someone else is controlling it. For example, the following context of someone being in a constant have' relation with the theme but then performatively creating a new have $_{P}^{\prime}$ relation for the theme is not naturally describable by a simple past tense expression of new possession as with give, though notably futurate expressions are possible (including present tense futurates), expected since there would be no linguistically entailed clash of possessional relations:

(75) [ Mary is writing something with a very nice pen, and John, who is watching her, remarks on how nice it is. She decides then and there to give it to him, and says... ]
a. I'm going to give it to you!
b. I'll give it to you!
c. Take it!
d. It's yours!
e. ??I just gave it to you!

Even if in Mary's mind she has officially and decidedly released alienable possession of the pen and it is now John's, the give construction in (75c) is not an entirely felicitous way of describing this situation while she is actively using the pen. Thus a clash between the manner-of-motion of continuous application of 
force roots and the meaning (or pragmatics) of the indirect object construction would explain their dispreference for indirect object templates, something that would only arise if the root entailed a certain sort of possession to begin with, in violation of Bifurcation.

Of course, this argument has presumed that somehow the natural interpretation of the possession relation of the template is have $e_{p}$. But assuming other types of possession will derive the same prediction. If the possession from the template is instead have ${ }_{C}^{\prime}$ then a similar clash applies since in general it is unusual for two individuals to have ${ }_{C}^{\prime}$ the theme at the same time, especially when only one is actually holding or otherwise physically manipulating it. The other two types of possession identified in $\$ 3.2$ are inalienable possession, which is independently unusual for a continuous application of force verb (which typically describes physically moving an actual artifact somewhere), and focus possession, which could in principle be any type of relationship between the recipient and the theme, including those that may not clash with the have ${ }_{C}^{\prime}$ relation we claim comes from the root. In this case a continuous application of force predicate should be fine for at least some such contextually-defined relations, and this does seem to be the case. For example, the following is acceptable in a context where the causer is bringing a chair to the recipient for her to sit on:

(76) John dragged Mary a chair to sit on.

Similarly, (75e) is significantly more acceptable if the intended reading is (for example) that Mary has decided to assign this particular pen to John for him to be in charge of cataloging when their company does office supply inventory later in the week but she is otherwise not releasing alienable possession or control of the pen to him. Crucially, these facts all depend on the idea that the roots of continuous application of force verbs must entail some kind of possessional semantics (have ${ }_{C}^{\prime}$ relating the causer and the theme) for any of this to arise, meaning in turn that it has possession as part of its meaning despite the fact that it does not entail new possession of the theme by the recipient. Thus again a templatic notion wholly independent of the template arises from a given root, arguing once again against Bifurcation.

\subsection{Alternative analyses: Allosemy and allomorphy}

So far all of the evidence strongly suggests that roots directly entail templatic notions such as possession, co-location, and change of state, contra Bifurcation. However, in this section we briefly consider an alternative analysis of the data above that is consistent with Bifurcation, and argue that while technically possible 
it nonetheless suffers from several drawbacks that make the analysis we have proposed preferable. In particular, it could be that all of the relevant (prospective and actual) possessional and (prospective and actual) co-locational states, plus anything else that seems templatic that is conditioned on the root, in fact does arise from the template rather than the root under some type of root-conditioned allosemy. For example, we could assume that the two $\mathrm{P}$ heads and also $v_{\diamond \text { cause }}$ can have different meanings depending on what root is head-adjoined to $v_{\diamond \text { cause }}$ (building on an idea initially pointed out to us by Asaf Bachrach, p.c., and echoing a related discussion on change-of-state verbs in \$2.5.2 rooted in Myler 2014 and Wood and Marantz 2015). On this analysis the $\mathrm{P}$ heads could describe, in addition to the have' and $R^{\prime}$ states each currently entails, other states depending on the root, and there could be variation in whether the causal $v$ head has or does not have the modality. To put it more generally, perhaps the indirect object and to constructions do not arise from two very general templates but rather represent a suite of templatic ambiguity keyed to particular roots. Alternatively, an allomorphy analysis would be effectively equivalent: there would be several templatic $\mathrm{P}$ and causal $v$ heads that are all morphologically identical but with different meanings and conditioned to occur with different roots.

While such analyses would presumably be technically possible and would be consistent with Bifurcation, they have several undesirable traits. First and foremost, as Beck and Johnson (2004: 108-10) argue, ditransitives allow restitutive again - the result but not necessarily anything else held before-something expected from event templates that separate out result states as separate constituents again can attach to (here projections of the result-denoting $\mathrm{P}$ heads). Consider such a case in (77) for a ballistic motion verb in the to-construction, where there is an acceptable reading in which a flower has always been in a given location, John removes it from that location, and then he tosses it back there again:

[ A plant grew on the opposite side of a rock from where John is standing. He picks it, then tosses it back to its original location. ]

John tossed the plant [ to the other side of the rock again ].

A couple of observations are in order. First, actual co-location seems to be what is presupposed to have occurred before, not prospective co-location, which would be what the templates provide on our own analysis in the preceding sections would predict. In particular, the state derived by attaching again to $\mathrm{P}_{\text {loc }}{ }^{\prime}$ would be that the recipient is in an $R^{\prime}$ relation with the theme and it is presupposed that this held before, all of which would then be embedded under the prospective caused change introduced by $v_{\diamond \text { cause }}$ : 


$$
\begin{aligned}
& \exists v\left[\text { effector } ^ { \prime } ( \mathbf { j } , v ) \wedge \diamond \exists e \left[\operatorname { c a u s e } ^ { \prime } ( v , e ) \wedge \exists s \left[\operatorname{become}^{\prime}(s, e) \wedge R^{\prime}(\mathbf{b}, \mathbf{m}, s) \wedge\right.\right.\right. \\
& \left.\left.\partial \exists e^{\prime \prime}\left[e^{\prime \prime} \ll s \wedge R^{\prime}\left(\mathbf{b}, \mathbf{m}, e^{\prime \prime}\right)\right]\right]\right] \wedge \text { tossing }^{\prime}(v) \wedge \exists e\left[\operatorname{cause}^{\prime}(v, e) \wedge\right. \\
& \exists s\left[\text { become } ^ { \prime } ( s , e ) \wedge a t ^ { \prime } \left(\mathbf{p}, \mathbf{j}, \text { init }^{\prime}(e) \overline{) \wedge \exists z\left[a t^{\prime}(\mathbf{p}, z, s) \wedge z \neq x\right]\right]\right] \wedge}\right.\right. \\
& \left.\diamond \exists e\left[\operatorname{cause}^{\prime}(v, e) \wedge \exists s\left[\text { become }^{\prime}(s, e) \wedge a t^{\prime}(\mathbf{p}, \text { o.s.o.r, } s)\right]\right]\right]
\end{aligned}
$$

However, this hinges on the pragmatics of the situation, where context establishes actual co-location. Consider a context where the flower had never been on the other side of the rock, and first Mary tries to drop it there-thus establishing a prospective co-location state in context-but the wind blows it out of the way so that the state never arises. If John tosses it to the other side of the rock the example sentence in (77) is still acceptable, meaning prospective prior co-location is sufficient for a restitutive reading.

A more critical point, however, is that release roots like $\sqrt{\text { Toss }}$ in (77) also give rise to an actual loss state, as argued in \$3.6.2 and as represented in (78). On the allosemy (or allomorphy) analysis that puts the states together as all originating in the $\mathrm{P}$ heads this actual loss state would also be introduced by $\mathrm{P}_{\mathrm{loc}}$, and thus should be under the scope of again. Yet this is not the case-it is not a requirement in (77) that John have actually or even prospectively released the flower before, or that anyone has in fact (as in the original context in (77)). This suggests that the rootentailed result state of lack of possession is introduced above $\mathrm{P}_{\text {loc }}$ in the structure (e.g. on $v_{\diamond \text { cause }}$, as we have proposed above).

In the case of toss, however, the root-entailed actual result does not subsume the templatic one (though the additional prospective result introduced by toss that the theme end up with the goal/recipient does). A reviewer asks about roots that ensure that the template-entailed state does obtain, such as accompanied motion verbs. Consider the following in the given context:

(79) [ Mary was carrying a box to the security council meeting but security guards stopped her right before she got it in because she did not have the right security clearance. John, who has the appropriate clearance, picked up the box and carried it to the council meeting instead.] John carried the box [ to the security council again ].

The reviewer suggests that this sentence is not entirely acceptable in this context, a judgment with which we concur. This might suggest that what again is scoping over in at least this case must include actual arrival, putting the supposedly rootentailed result somehow in $\mathrm{P}_{\mathrm{loc}}$ as the $\mathrm{P}$-allosemy (or allomorphy) analysis under discussion would suggest it should be. However, this is again a pragmatic issue: as discussed in $\$ 3.3 .2$, whatever the relevant modality is here, there seems to be some requirement that the template-entailed result state be possible, yet the security clearance issue would preclude that. Consider an alternative context where Mary 
has the required clearance and was carrying the box to the council, but was struck by a vehicle and instead ended up in the hospital (i.e. the result was preempted rather than wholly precluded). If John retrieves the box and successfully carries it to the security council, the example sentence in (79) is acceptable.

Thus again the supposedly root-entailed result state of actual co-location is outside the scope of again. And, of course, were the box originally constructed at the security council office, later removed, and then carried back there by John-a canonical restitutive context - then the example sentence in (79) is also acceptable. In this case, the theme was at the location prior to the event, though note that the root-supplied co-location result state for carry and other accompanied motion verbs also requires the effector participant to be there as well, and this is not under the scope again, further evidence that the root result is not under again on a restitutive reading.

Indirect object templates also license restitutive modification. Here the core of the relevant state is possession, and in this case it is a bit difficult to set up contexts for restitutive readings since we need a type of possession that always held or otherwise never came about per se. The best candidate for this would be some type of inalienable possession. In such a context it is possible to license a restitutive reading where the relevant possession had always held before and never arose:

(80) [ Sandy lost her right hand in an accident, and a doctor attaches a replacement. ]

The doctor gave Sandy [ a right hand again ].

Here there was no prior giving, suggesting that $\sqrt{\text { GIVE }}$ and its various lexical entailments are outside the scope of again. However, in this context the prior result state of possession had obtained before. Yet this does not mean that somehow that particular aspect of what we had said in $\$ 3.6 .1$ is the meaning of $\sqrt{\text { GIVE }}$ is somehow under the scope of again. For example, possession that does not obtain at the real world can also license restitutive modification. Consider a context where Parliament has passed a law by which any future children of the current queen are barred from inheriting the throne. The queen then has a child, who is born not possessing that right but for whom the right is arguably licensed in some alternative world in which that law was never passed. Parliament then passes a law rescinding the prior law, thus giving the daughter a right to the throne. In this case a restitutive reading is licensed:

(81) Parliament gave the queen's daughter [ her right to the throne again ].

Indeed, another way to describe this would be Parliament restored the queen's daughter's right. Thus what is under the scope of again is purely what we have claimed is coming from the template, while the actual possession state is not under 
its scope. This once again argues against an allosemy (or allomorphy) analysis that puts all of the relevant states in the P heads. In sum, the data presented here argue against analyses where $\mathrm{P}$ heads introduce all of the states relevant for a given ditransitive.

Still further, maintaining the clean split between a state-denoting templatic $\mathrm{P}$ head and a $v$ head entailing (prospective) caused change faces more general difficulties, since for most, if not all, of the ditransitives discussed above there is a split among the result states in terms of which are prospective and which are nonprospective. For example, throw in the indirect object construction entails possible having but actual losing. Since there would be one locus of prospectivity-distinct senses or allomorphs of the causal $v$ head-any modality introduced by $v$ would outscope all result states conjunctively introduced by $\mathrm{P}$, such that all results would have to be prospective or be non-prospective. To maintain the mixed status of the results additional complexity would be needed. Probably the simplest analysis would be that the $\mathrm{P}$ heads only introduce the have' and $R^{\prime}$ states, as on our own analysis-which would solve the problems with again above-and the various allosemic (or allomorphic) variants of $v_{\diamond \text { cause }}$ introduce a modality scoping over the states introduced by $\mathrm{P}$ and in addition introduce all of the various additional non-prospective and prospective root-conditioned results on a root-by-root basis. This would effectively, though, recreate the analysis we have proposed above: the adjoined combination of the root and $v_{\diamond \text { cause }}$ would have exactly the truth conditions we have proposed above, save that instead of the root contributing the root-specific information $Q^{\prime}$ about the theme and the recipient $v_{\Delta \text { cause }}$ does so, contingent on which root is adjoined to it. This is ultimately a roundabout way of saying that certain meaning is introduced by virtue of the root without saying the root itself has that meaning. This raises the question of what, other than a desire to maintain Bifurcation, would motivate such an analysis.

Finally, focusing on the allomorphy variant of this analysis we would expect that in at least some languages the relevant specialized meanings of the $\mathrm{P}$ and $v_{\diamond \text { cause }}$ heads-whichever it is that introduces the special meanings-would be lexicalized in overt morphology in at least some language. However, we are not aware of any such lexicalizations in any languages. Causal morphemes are rarely if ever so specific in their meanings so as to include notions like releasing or contractual obligation that we are aware of (see e.g. typologies of causal morpheme meanings as discussed in Wierzbicka 1998, Talmy 2000: 471-550, Shibatani and Pardeshi 2001, Wolff 2003, Wolff and Song 2003, Wolff et al. 2005, inter alia, mostly centered around degrees of directness of causation and force-dynamics). Similarly, the overt morphology that most closely corresponds to the $\mathrm{P}$ heads here would be applicative morphology, and indeed syntactified event structural analyses of ditransitives such as those in Pylkkänen (2008: 12-9) and Bruening $(2010 a, b)$ have assumed that the relevant templatic element is applicative in nature. However, languages with overt applicatives do not, as far as we are aware, have 
such highly specialized applicative meanings. Some languages may have just one applicative morpheme that covers all applicative functions in the language (as with Lubukusu as per Peterson 2007: 6-14). Languages that do have multiple specialized applicatives still do not distinguish such fine-grained meanings. For example, as discussed in Jerro (2016), Kinyarwanda has two major applicatives: -ir and $-i s h$. However, their meanings are quite broad, where -ish covers instruments and causees while -ir covers a broad spectrum of beneficiary, recipient, and locational notions of various stripes, making none of the fine-grained distinctions required on an allomorphy analysis of the data we have discussed above. Peterson (2007: 17) attributes seven applicatives to Hakha Lai, but their meanings are fairly general (e.g. benefactive/malefactive, comitative, instrumental) and do not have the level of deep granularity required to account for the data here (see also Peterson 2007: 40-5 on languages with just one applicative morpheme vs. those with more).

In sum, while an allosemy or allomorphy analysis may be technically possible, it would involve otherwise unmotivated technical assumptions to essentially recreate our own analysis, while making morphological predictions that are not borne out. The analysis we have proposed works with fewer assumptions, and mostly just hinges on assuming that roots differ idiosyncratically in their meanings in ways expected within the lexicon and that their meanings interact through wellestablished semantic properties to produce the right results. ${ }^{23}$

\subsection{Root vs. template in ditransitive verbs}

Now that we have established that roots introduce the same meanings as the templates they occur in do, we must again ask the question we asked in $\$ 1.4 .3$ and $\$ 2.6$ of whether there is a need for a root vs. template distinction among ditransitives given that the roots seem to have meanings in them that in some cases wholly subsume the templates in which they occur. As with previous verb classes, we suggest once again that the templates do in fact have significance independent of the root. The most obvious way of course is that it is a very clear constructional effect of the indirect object construction that it introduces a (prospective) possessional semantics not coming from the root, the root sensitivity effect of $\$ 3.3 .1$. But more significantly, ditransitives provide especially clear evidence for a grammatical effect of templates that roots do not give rise to even when the same meanings are introduced.

\footnotetext{
${ }^{23}$ As noted in fn. 10, if there is a $v_{\text {become }}$ head in the structure, then that head could be another possible attachment site for manner roots above the state-denoting head and another attachment point for again on a repetitive reading. Furthermore, it would also open up another point for positing possible allosemy/allomoprhy. However, it should be clear that this would not solve any of the issues discussed here, and for allomorphy might explode the problem.
} 
In particular, recall Harley's full event structures for ditransitive verbs in (3), repeated here (modulo updated names for the templatic heads):

(82) a.

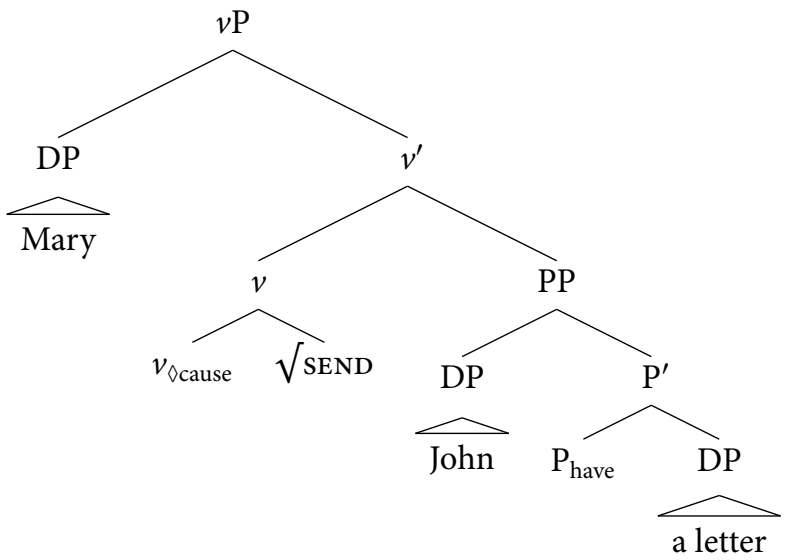

b.

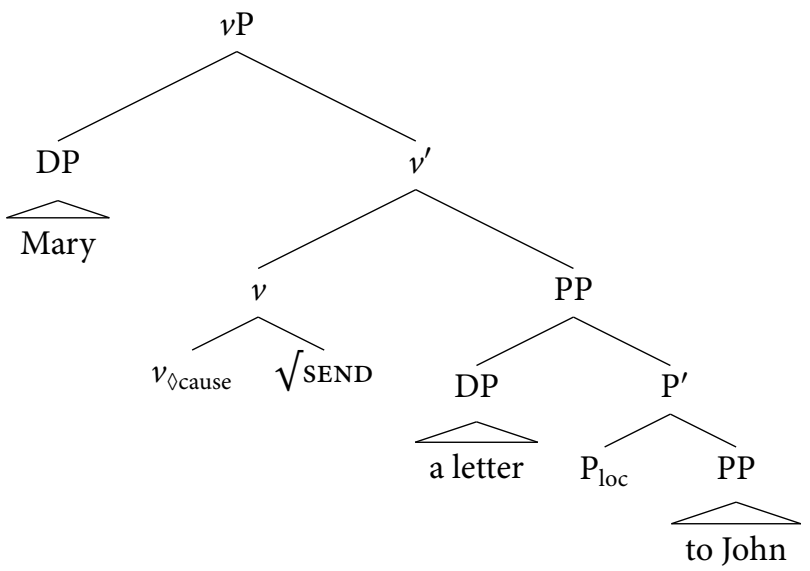

As discussed in $\$ 3.1$, these event structures not only capture the London Office effect and the argument realization facts, they also capture the well-known binding asymmetries of Barss and Lasnik (1986), whereby the first complement can bind into the second while the reverse is not possible:

(83) a. Kim sent [ every employee $]_{i}\left[\right.$ her $_{i}$ paycheck ].

b. *Kim sent [ its ${ }_{i}$ employee ] [ every paycheck $]_{i}$.

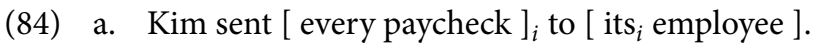

b. ${ }^{*}$ Kim sent $\left[\text { her }_{i} \text { paycheck ] to [ every employee }\right]_{i}$.

This follows because the $\mathrm{P}$ heads define different asymmetric c-command relations

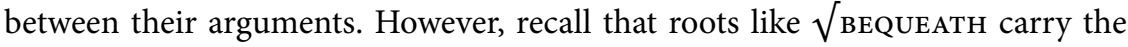


same meaning as the indirect object template, which thus surfaces even in the to construction. Since the meaning is the same as the indirect object template does this mean this root should give rise to the same asymmetric binding facts? This is not the case. In the to construction with bequeath the c-command facts in (85) are the opposite of the indirect object construction in $(83 \mathrm{a}, \mathrm{c})$ and instead are in line with other to constructions as in $(83 \mathrm{~b}, \mathrm{~d})$ :

(85) a. Kim bequeathed [ each account $]_{i}$ to its ${ }_{i}$ associated heir.

b. Kim bequeathed his ${ }_{i}$ account to [ every associated heir $]_{i}$.

Thus while possessorhood can be introduced by the root or the template, it seems that only template-introduced possession corresponds categorically to a specific type of relative argument prominence (just as with subject/object c-command asymmetries for transitives as per $\$ 1.4 .3$ ). This suggests a role for templates not found with roots. We return to this issue in $\$ 4.5 .4$ and show that ditransitive templates are also unique among the components of their event structures in defining scopal positions for again, though we must first discuss the issue of manner in the roots of ditransitives.

\subsection{Concluding remarks}

In the above we have seen seen several interactions between roots and templates that all suggest that roots entail templatic information such as possession, arrival, and change of state:

- Root sensitivity: Roots determine how each template is interpreted, sometimes causing one template to take on the meaning of another.

- Root telicity: Roots entail transitions (sometimes entirely distinct from the template) and thus telicity.

- Root durativity: Roots determine scalar complexity and thus durativity.

- Root-determined argument realization: Roots determine which templates they may occur in owing to the roots' semantics.

Key to analyzing all of these phenomena is that various templatic meanings must be entailed by the root: these roots spell out states in the template, define aspectually significant results that figure into telicity and durativity, and determine argument realization through clashes in the templatic meaning coming from the root and that coming from the template. These results thus argue against Bifurcation of root and template meanings. Perhaps the core analytical proposal underlying all of these facts has to do with the claim that the meanings of the two ditransitive templates are actually quite light in nature, and thus it must be the 
root that is filling in sufficient information to derive the various phenomena listed above. We thus more specifically developed an analysis in which ditransitive roots are primarily manner-defining and adjoin to $v_{\diamond \text { cause }}$ but also define information about the final state of the theme and recipient in the event being described that in some cases subsumes and in some cases augments-or even is augmented bywhat the template entails, an analysis we suggest is superior to analyses that would attempt to capture the same facts while preserving Bifurcation. The resulting classification of roots in turn derives a verb classification that makes predictions about the interpretation of the two variants in the dative alternation and also whether the alternation arises at all, in a way that is technically orthogonal to what the templates bring to the event structure though interacts with it, something we return to in in $\$ 5.4$.

Now, as with the roots of change-of-state verbs in Chapter 2, we still have not discussed why the roots of ditransitive verbs might have these properties. In $\$ 5.2$ we return to this question and suggest that to a large degree it has to do with the ways certain types of actions described by certain manner-adjoined roots are conceived of as having as an inherent part of their definitions that certain changes come about. First, though, we examine the second major question of this study, namely how many types of idiosyncratic meaning a root can have and the validity of Manner/Result Complementarity as a hypothesis that places constraints on possible root meaning. Along the way we also develop a final argument against Bifurcation, focusing on entailments of causation. 


\section{4 \\ Manner/Result Complementarity and causation in verbal roots}

\subsection{Manner/Result Complementarity}

In the preceding two chapters we argued that several types of templatic meaning must be found in the meanings of roots, a violation of the Bifurcation Thesis. These include uncontroversially templatic entailments such as change-of-state and possession, but also entailments such as co-location and entailments that give rise to aspectual properties such as durativity and telicity, otherwise typically (though perhaps not universally) thought of as arising from certain templates. The result then is that templatic meanings are not excluded from the meanings of roots. ${ }^{1}$

However, an alternative type of limitation on possible root meanings is that they are limited in how much idiosyncratic, non-templatic meaning they can entail. One version of this question would examine whether there are types of idiosyncratic meaning that are too complex. We know that roots can describe actions and describe states but are there limits on how complex those actions and states can be in terms of how many truth conditionally distinct lexical entailments are needed to define them? Grimshaw (2005: 85) has suggested that this is not the case-a single given action or state can be as complex as is appropriate for what is being described. But an alternative constraint on idiosyncratic meaning is that roots can only describe a limited number of types of idiosyncratic meaning, e.g. just an action or just a state but not both.

In this chapter we suggest that there are no such limits on root meanings of this sort, either. Our case study is the roots of verbs that in prior literature have been suggested to violate so-called Manner/Result Complementarity, the proposal first articulated in Rappaport Hovav and Levin (2010) (based on observations dating back at least to Levin and Rappaport Hovav 1991) that has as a component the hypothesis that a verb's root may only describe a manner (e.g. a type of action) or a (result) state, but not both simultaneously (for discussion see Rappaport Hovav and Levin 2010; Goldberg 2010: 46-50; Husband 2011; Mateu and Acedo-Matellán 2012; Levin and Rappaport Hovav 2013; Rissman 2016; Beavers and KoontzGarboden 2017a). We argue that this is not in fact the case, and that there are

1 This chapter is a revision and expansion of Beavers and Koontz-Garboden (2012). 
verbs that entail both a specific action and the specific result it brings about (see also Goldberg 2010: 46-50; Rissman 2016; Beavers and Koontz-Garboden 2017a), and furthermore this is due to the roots of such verbs themselves entailing both notions at the same time.

To make our case, we first outline what Manner/Result Complementarity is and what supposedly gives rise to it. The roots of Manner/Result Complementarity stem from Rappaport Hovav and Levin's claim that eventive verbs fall into two broad semantic classes: those entailing the manner in which some action is carried out as in (1a), and those entailing the coming about of some particular result state as in (1b):

(1) a. Manner verbs: run, walk, swim, jog, blink, yell, scrub, wipe, sweep, etc.

b. Result verbs: break, shatter, crush, destroy, dim, clean, etc.

Rappaport Hovav and Levin (p. 25) crucially claim that no single surface verb entails both at the same time. Instead, the manner in which something comes to be in some state is unspecified for result verbs-a breaking can be accomplished in any number of manners (e.g. snapping, slapping, etc.) - while the result is unspecified for manner verbs-one can run around and end up back where one started (or run in place). Of course, a complex verbal predicate consisting of two clearly separate overt lexemes can entail manner and result simultaneously (e.g. resultatives like sweep clean). Rappaport Hovav and Levin's claim is (descriptively) that a single surface verb never entails both.

Rappaport Hovav and Levin propose that this complementarity follows from how event structures are composed, focusing on the number, type, and place of lexical semantic roots. In their formulation, a single lexical semantic root can either modify an underlying action-denoting event structural operator to describe an action or be an argument of an underlying change-denoting operator to describe a result state, but not do both simultaneously. Furthermore, their discussion presupposes that there is only ever one idiosyncratic root object per single surface verb. Cashing out these assumptions in terms of the types of syntactified event structures we have adopted in the preceding chapters, this would mean that an idiosyncratic root object can only occur in manner position as an adjunct to a causal or action defining $v$ head (as per \$3.5) or as the complement of some $v_{\text {become }}$ (as per $\$ 2.2$ ), deriving $(2 \mathrm{a}, \mathrm{b})$ and $(2 \mathrm{c})$ respectively. The difference between (2a) and (2b) is in whether the highest $v$ is a $v_{\text {act }}$ head defining a pure action with no result or a $v_{\text {cause }}$ head defining causation plus some other functional projection XP as its complement (such as a $\mathrm{PP}$ as in the ditransitive structures in Chapter 3). In the latter case, the additional XP defines the result state, such 
that the root itself is free to be a manner adjoining root. However, a root cannot simultaneously be an adjunct to a $v$ and a complement to $v_{\text {become }}$ (however that might be analyzed), nor can a single surface verb have two roots, each in either position, ruling out $(2 \mathrm{~d}, \mathrm{e})$ respectively:

(2) a.

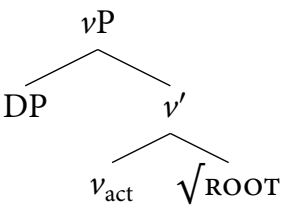

b.
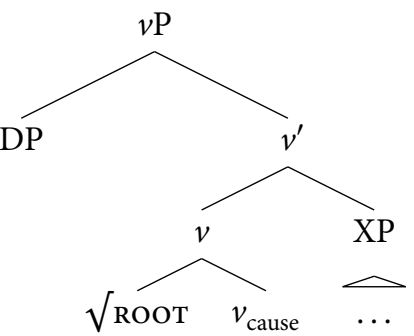

c.

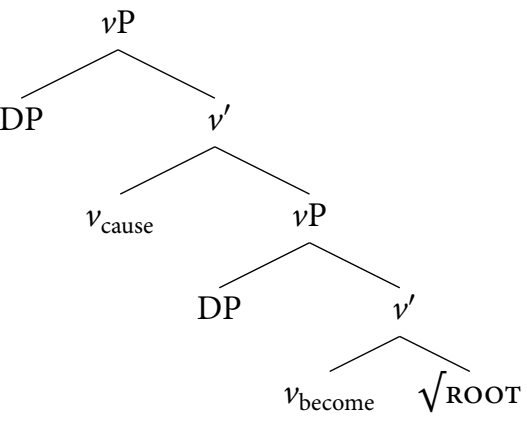

d. ${ }^{*}$

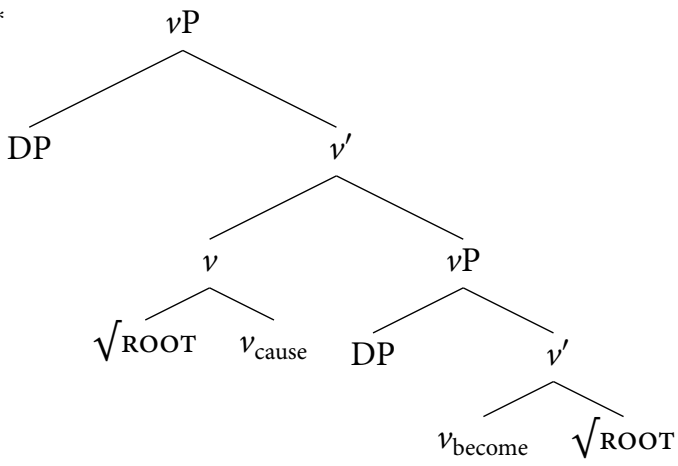




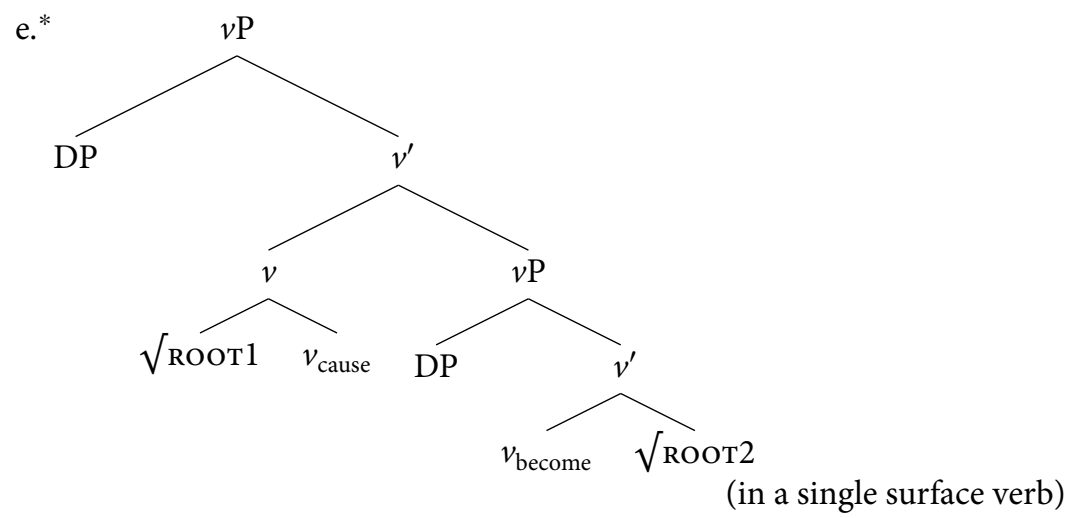

Assuming manner-adjoined roots only describe manners and result complement roots only describe result states, from this it should follow that a verb will either entail a result or entail a manner, but not both simultaneously.

Here we first examine the empirical and theoretical foundations for Rappaport Hovav and Levin's proposal. We argue that the Manner/Result Complementarity question has been complicated by the use of diagnostics for manner and result as components in a verb's meaning that are (a) interdependent in ways that make them inappropriate for verifying complementarity and (b) not linked to either truth conditional or event structural semantics in an explicit way. Thus a significant portion of this chapter consists of devising and reviewing independent truth conditional diagnostics for identifying manner and result in a verb's meaning, and comparing them with standard diagnostics such as sublexical modifiers like again that probe for event structure. We argue that once the diagnostics are so delineated, the question of Manner/Result Complementarity in fact becomes two questions, one about truth conditional content and one about event structure.

Crucially, we show that in truth conditional terms there is evidence against Manner/Result Complementarity. We focus in particular on three major classes of verbs: verbs of manner of killing (e.g. guillotine, drown) (Levin 1993: 230-3, Krohn 2008), manner of cooking verbs (e.g. sauté, braise) (Levin 1993: 243), and ditransitive ballistic motion verbs (e.g. throw, toss) (Gropen et al. 1989: 243-4). In addition to using manner and result diagnostics to show that these verbs entail both a manner and a result simultaneously, we also use evidence from scopal modifiers to show that verbs cannot have both a root describing a manner and a separate root describing the result state simultaneously, so that Manner/Result Complementarity does hold as a fact about event structures. To accommodate truth conditional manner+result verbs there must therefore exist a class of roots in which manner and result meanings are "packaged together" as a single unit: the root itself entails a change into a specific state and a specific action that caused that change-of-state. The conclusion then is that root meanings may be quite complex 
and involve both manner and result at the same time, contra Manner/Result Complementarity. Thus there are no limits on how much meaning a root can have qua how many types of idiosyncratic meanings it may lexically entail. Admitting a class of manner+result-describing roots to the typology of possible roots of course in turn derives a new class of verbs, manner+result verbs, further extending our theory of root-determined verb classes, which we return to in $\$ 5.4$.

Once we have established this, though, we then show that the same data also provide a final argument against Bifurcation. In particular, the semantic notion that relates the manner and result together in the verbs we look at is causation, which furthermore must be coming from the root as well, and scopal data we discuss below bear this out. Given that causation is the meaning of $v_{\text {cause }}$ then it appears that yet another templatic meaning can be part of roots. In fact, taking the meaning of $v_{\text {cause }}$ into account alongside the meanings of all the other templatic heads we have considered in previous chapters, it appears that any templatic meaning can be found in some root.

We begin in $\$ 4.2$ by outlining a series of diagnostics for whether a verb entails result, and in $\$ 4.3$ we outline diagnostics for whether a verb entails manner, demonstrating that canonical result and manner verbs are distinguished by these diagnostics. In $\$ 4.4$ we apply these diagnostics to manner of killing verbs, cooking verbs, and ballistic motion verbs, and show that they pass diagnostics of both types and thus rightfully should be classed as manner+result verbs, i.e. verbs entailing a manner and a result simultaneously. In $\$ 4.5$ we examine complementarity in event structures, and use evidence from scopal operators to suggest that in manner+result verbs the two meaning components are packaged together in a single scopal unit, rather than being introduced by two separate roots. This yields a type of complementarity, albeit one requiring a class of roots describing both a manner and a result state simultaneously. In $\$ 4.6$ we justify that causation is part of the root meaning, another violation of Bifurcation. We also suggest that the fact that the roots of manner+result verbs entail causation may partly explain why these verbs entail manner and result at the same time, with each filling in the causing and caused events respectively that together define a causal relation. We discuss the need for templates in manner+result verbs despite the complexity of the root meanings in $\$ 4.7$, and offer some concluding commentary in $\$ 4.8$.

Before we begin we offer a few methodological preliminaries. First and foremost, for purposes of understanding Manner/Result Complementarity we take as our object of study surface verbs that consist of only one overt idiosyncratic root morpheme (though there may be functional, derivational morphemes such as inchoative or causative morphemes). We focus on verbs with a single overt idiosyncratic root because within syntactified theories of event structure most if not all verbs are analyzed as being polymorphemic in the sense of being the surface realization of single root object and at least one but possibly more functional heads applying a category to that root and/or introducing additional 
event structural meaning (not including inflectional functional heads such as $\mathrm{T}$ ). An event structure resulting in a verbal form with two surface idiosyncratic roots would necessarily involve two root objects in such an architecture, and in this case one root could contribute manner and one result. This would correspond to a resultative like sweep clean, but nobody has ever claimed Manner/Result Complementarity for such structures (obviously) and so they are not of interest to us except as a comparison case. Rather, we are interested in whether a verb that is not obviously composed of multiple surface roots can entail both manner and result simultaneously.

Crucially, if there is just one overt idiosyncratic morphological root for a given surface verb this could either mean that there is only one root in the event structure or that there is more than one root but one has not had any impact on the surface morphological form of the verb, i.e. it has null instantiation but otherwise the verb's event structure is exactly like that of a resultative. It will be a key point below showing that in such cases there is in fact just one root in the event structure and not more, ultimately leading us to conclude that in cases where such a verb does entail manner and result simultaneously both the result state and the action leading to it must be lexically entailed by a single root. Finally, as per $\$ 1.4 .1$ we assume that a given surface verb may be polysemous, and this may arise from one of two sources: the same root in different event templates or the same template but with different roots (perhaps even different senses of the "same" root and/or template). However, we take Rappaport Hovav and Levin's Manner/Result Complementarity hypothesis to be a constraint on individual event structures, and thus in cases where polysemy could be justified we focus on just one reading with a single event structure, though we return to polysemy briefly in $\$ 4.4 .4$.

We now develop a suite of result and manner diagnostics, motivated by comparing their application to supposedly canonical result and manner verbs and showing that they draw a distinction. For result verbs we use destroy, break, shatter, clean, and dim, and for manner verbs we use unergatives run, jog, yell, and blink and transitive surface contact verbs scrub, sweep, and wipe, all of which we take to be uncontroversially result or manner verbs. We will then apply our tests to verbs we claim have both manner and result in their meaning and thus represent manner+result verbs. Finally, two caveats are in order. First, as we discuss in $\$ 4.3$, prior classifications of manner and result verbs may have been built on diagnostics that preclude the possibility of manner+result verbs. Thus verbs previously classified as either manner or result may actually be manner+result verbs, or otherwise misclassified. Our goal is not to redo prior classifications, but rather to demonstrate the existence of a third, mixed class, thereby demonstrating that root meanings can be richer than generally believed. Second, there is definitely individual variation in speaker judgments on some of the cases we consider, especially-in the case of verbs that entail result-what exactly the result is. Crucially, the exact nature of a given result (or manner) is not important. For 
example, one major class we consider are manner of killing verbs, which we argue entail both a manner and a result, and furthermore to our intuitions the entailed result is death for the verbs we discuss. However, our claim - that these verbs entail both a manner and a result-is valid so long as they entail some result, death or otherwise. For example, we consider the verb guillotine and our intuitions are that one cannot survive a guillotining. But we might consider a mythological creature such as a zombie that could in principle survive a guillotining, though it has still at least lost its head (or some appendage; see fn. 18) or is otherwise injured. Thus there is still a result. Similarly for crucify, while we have strong intuitions that death must ensue, were one to claim it did not, there is still uncontroversially a change of location (being hung up in a particular configuration), and thus a result. One verb where we have seen an especially high degree of equivocation is electrocute. We and other speakers we have consulted believe it must describe a death, while many others (including one reviewer) have suggested it has a meaning more like shock, with no particular result state (the Oxford English Dictionary offers both definitions). For the latter set of speakers, it is simply not a result verb (see $\$ 4.4 .1$ ), and for many verbs we discuss it could be that for some speakers it lacks the relevant meaning component under discussion even if for others it has it, expected in speaker to speaker variation in lexicalization (see \$2.2.3). Overall, the goal is to demonstrate that verbs entailing both manner and result do exist for some speakers, even if the exact truth conditions of a given component and the broader classification may differ between speakers.

\subsection{Result entailments in verbs}

We begin by outlining a variety of diagnostics for demonstrating that a verb has result in its meaning, drawing partly on the discussion in Chapter 2 about whether roots have change in their meaning but applying the concept to surface verbs more specifically. The relevant notion of result as proposed by Rappaport Hovav and Levin (2010: 28) is the notion of scalar change, i.e. change of the patient in some state along a scale of possible states as per $\$ 1.6 .1$ and as utilized in our analyses of transitive and ditransitive change-of-state verbs in the previous two chapters. However, we make one terminological clarification regarding this notion: while Rappaport Hovav and Levin (2010) define result as entailing change along a scale, intuitively a result verb should be defined as a verb that gives rise to an entailment of some entity being in a different state at the end of the event than at the beginning, and indeed our first result verb diagnostic hinges on this. Yet as we demonstrated in Beavers and Koontz-Garboden (2017a), entailing change along a scale and entailing a new state of some patient at the end of the event are not the same thing. Some classes of verbs-in particular motion verbs like climb and circle that entail change along a path but where the path may double back to the moving figure's 
original position-entail measurable change along a scale (corresponding in this case to a path) but do not entail a new final state of the patient distinct from its state at the beginning of the event (e.g. in the model of scalar change of motion verbs outlined in $\$ 3.5$ it could be because there is change along a motion scale but the corresponding locations in the actual world are identical between the beginning and ending states). For this reason Beavers and Koontz-Garboden distinguished scalar change more broadly, the entailment of change along a scale over the course of the event regardless of whether it gives rise to a new final state, from scalar result, which is change along a scale over the course of the event that does result in a new final state of the patient. We focus on entailments of scalar result below, and thus largely ignore scalar change that lacks scalar result. However, as we discuss extensively in Beavers and Koontz-Garboden (2017a), scalar change lacking scalar result can occur in verbs that also entail manner, and thus there are verbs within that domain that violate Manner/Result Complementarity in the truth conditional sense as well.

\subsubsection{Result diagnostic \# 1: Denial of result}

In an event of change along a scale that does not somehow double back on itself (i.e. scalar result), the patient necessarily has a different degree on the scale at the end of the event than at the beginning. As such, it will be contradictory to follow any predicate headed by a result verb with a denial that the patient has undergone a change in some named property, as expected per the analysis of scalar change in \$1.6.1. ${ }^{2}$ As Beavers (2011b: 341-4) suggests (following Kratzer 2000), one specific version of this test is to see if denying the past participle form of the verb applied to a DP coreferential with the putative patient yields a contradiction, as with uncontroversial result verbs in (3) (where the pronoun is to be interpreted as coreferential with the argument being tested for patientivity, here the object in the first clause): ${ }^{3}$

(3) a. \#Shane just broke the vase e $_{i}$, but it ${ }_{i}$ is not broken.

b. \#Shane just shattered the bottle ${ }_{i}$, but $i_{i}$ is not shattered.

c. \#Shane just destroyed his house ${ }_{i}$, but $\mathrm{it}_{i}$ is not destroyed.

However, it could be that this diagnostic does not show that all of these verbs entail the same notion of result. Thus we might instead find some single,

\footnotetext{
${ }^{2}$ It is irrelevant whether the patient is the subject as with an inchoative or the object as with a causative, and thus we do not distinguish them below in terms the acceptability of our result diagnostics, unless a given diagnostic makes explicit reference to a certain grammatical function.

${ }^{3}$ We use just in the initial clause to mitigate against the change being done and then subsequently being undone; Kratzer (2000) uses instead $x$ is still VPed.
} 
non-verb-specific inference for all result verbs. Beavers suggests Something is different about $x$ for property change and creation/consumption predicates, where something is interpreted as an intrinsic physical property (see also Wolff 2003: 41, who gives something happened to $x$, citing Shibatani 1976: 2, (2); Beavers gives $x$ is somewhere else for motion verbs; see $\$ 4.4 .3$ ). Denying this also gives rise to a contradiction with canonical result verbs:

(4) a. \#Shane just broke the vase, but nothing is different about it.

b. \#Shane just shattered the bottle, but nothing is different about it.

c. \#Shane just destroyed the house, but nothing is different about it.

By contrast, prototypical manner verbs fail to generate a contradiction with continuations that deny a result. Precisely this point is discussed for surface contact verbs such as sweep, rub, and wipe by Rappaport Hovav and Levin (1998:101), who remark that:

"... none of these verbs, in its most basic use, entails a resulting change in the contacted surface. Thus, although a floor is typically swept in order to remove dirt and debris, a floor that is swept need not end up being clean. Although a hearer will infer that a swept floor is a clean floor because the conventional goal of sweeping is to clean a floor, there is nothing contradictory in saying Tracy just swept the floor, but there are still crumbs on it."

Of course, their continuation is a specific one pertaining to a specific verb (cp. result participles above). But the more general but nothing is different about $x$ has the same effect as in (5), and similarly with other canonical manner verbs such as unergatives as in (6):

(5) a. Tracy just swept the floor, but nothing is different about it.

b. Tracy just wiped the floor, but nothing is different about it.

(6) a. Bob just yelled, but nothing is different about him.

b. Bob just ran quickly, but nothing is different about him.

Thus, unsurprisingly, canonical result verbs have a result entailment, but not canonical manner verbs. ${ }^{4}$

\footnotetext{
${ }^{4}$ A reviewer questions this test with surface contact verbs, giving judgments like \#Bob just dusted/scratched/scrubbed/polished the table, but nothing is different about it. However, we reiterate our point at the end of $\$ 4.1$ that if a verb previously classified as a manner or result verb does not pattern as such according to our tests, the original classification may be incorrect. That said, regarding these specific verbs, dust could be lexicalized differently for different speakers, with a result meaning "to remove dust from" or a manner meaning "to touch with a duster" (or even "to remove dust by touching with a duster"), and similarly for scrub and polish (though to us the former is a manner verb and the latter a result verb). For scratch, we believe there are two homophonous lexemes, one that takes inanimate objects and refers to the result of producing a scratch, and one that takes animate (body
} 


\subsubsection{Result diagnostic \# 2: Object deletion}

Our second diagnostic is a set of related tests that comprise the bulk of those adopted by Rappaport Hovav and Levin, namely that transitive manner verbs but not transitive result verbs permit their objects to be omitted in certain contexts. Rappaport Hovav and Levin do not themselves link this directly to scalar change (or more properly scalar result), but Rappaport Hovav (2008: 24) proposes that scalar change verbs disallow object deletion since "... scales require that the participant whose property is measured out by them is overtly realized" (a conclusion that Beavers and Koontz-Garboden 2017a suggest applies more broadly to scalar change verbs and not just scalar result verbs). This condition may in turn follow from Rappaport Hovav and Levin's (2001: 779) Argument-per-Subevent Condition whereby there must be at least one overt argument for each distinct subevent in the verb's event structure. If scalar change involves a change-of-state subevent of which the patient is the sole participant, it must be realized (see Rappaport Hovav and Levin 1998: 122 for an earlier argument along these lines). Conversely, since transitive manner verbs do not entail scalar result (or scalar change more generally), there is no additional subevent beyond the manner, and the object may be deleted. ${ }^{5}$

The facts largely bear this out. An uncontroversial manner verb such as scrub in (7a) may readily occur intransitively as in (7b), giving rise to an existentially bound reading for the patient:

(7) a. Kim scrubbed the floor.

b. All last night, Kim scrubbed.

A second object deletion test is out-prefixation as in (8), where the default patient object is replaced by an object representing the agent of another event:

(8) a. Cinderella scrubbed the floor.

b. Cinderella outscrubbed her stepsisters.

Conversely, result verbs do not allow object deletion (the intended reading of the (b) sentences is object drop, not the inchoative):

part) objects and refers to a manner of skin contact. (See also Washio 1997: 12-6 for a similar point about wipe verbs in Japanese, where "removal" and "surface touching" senses are lexicalized into different, non-homophonous verbs.) What matters is simply that some verbs pattern one way with these diagnostics and others a different way.

${ }^{5}$ We present this diagnostic with the full awareness that it is not directly truth conditional in nature, since it relies on some set of principles linking truth conditional content to surface syntax, and it is the surface syntax we are discussing. However, since this was a proposed test in prior work we maintain it here for the sake of hewing closely to this literature. See Beavers and Koontz-Garboden (2017a: 864) for more discussion. 
(9) a. Kim broke the vase.

b. *All last night, Kim broke.

(10) a. Kim shattered the can.

b. *All last night, Kim shattered.

(11) a. Kim destroyed the house.

b. *All last night, Kim destroyed.

Similarly, although our judgments are less sharp, result verbs are also odd with out-prefixation:

(12) a. ??Kim outbroke the other vase-smasher.

b. ??Kim outshattered the other bottle-shatterer.

c. ??Kim outdestroyed the experienced wrecking crew.

Thus this test does seem to separate canonical result and manner verbs (though see Goldberg 2010: 47 for some counterexamples).

\subsubsection{Result diagnostic \# 3: Restricted resultatives}

Another diagnostic involves the range of possible resultative constructions the verb may appear in, for which manner verbs are generally less constrained than result verbs. For example, Rappaport Hovav (2008: 22) claims that "[a] verb with no lexically specified scale can appear with a variety of results. In contrast, verbs which have lexically specified scales... are very restricted in the kinds of resultatives they can appear with." ${ }^{\prime \prime}$ This test is illustrated in (13), where the manner verb scrub allows a range of result XPs predicated of the default object of $s c r u b$ as in (13a), plus XPs predicated of non-subcategorized objects as in (13b-d):

(13) a. Cinderella scrubbed the table clean/shiny/bare.

b. Cinderella scrubbed her knees sore.

c. Cinderella scrubbed the dirt off the table.

d. Cinderella scrubbed her house-cleaning competitors out of business.

This contrasts with result verbs like dim. While such verbs do allow result XPs, they must be compatible with the result entailed already in the verb, as in (14a,b)

\footnotetext{
${ }^{6}$ This distinction bears some similarity to the proposal of Washio (1997) that resultative constructions be split into what he calls "strong" and "weak" resultatives, the former roughly those that occur with verbs that do not independently entail a result, the latter with those that do. Among "weak" resultatives, the possible result XPs may be constrained by what change the verb already entails (see also Beavers 2011b: 343-4).
} 
for dim. Similarly, result XPs with non-subcategorized objects are generally not allowed, as in (14c): ${ }^{7}$

(14) a. Then the biologists dimmed the room to the level of starlight... $<$ https://archive.org/stream/naturalhistory11202unse/naturalhistory 11202unse_djvu.txt $>$ (Accessed May 8 2018)

b. *We dimmed the room empty. (Rappaport Hovav 2008: 23, (13d,e))

c. * Kim dimmed her eyes sore.

Other uncontroversial result verbs such as break are similar, where in $(15 \mathrm{a}, \mathrm{b})$ result XPs compatible with the entailed result are acceptable, but result XPs that are not are unacceptable as in $(15 \mathrm{c}-\mathrm{e})$, as are results with non-subcategorized objects as in $(15 f):^{8}$

(15) a. Kim broke the stick in half.

b. Kim broke the stick into pieces.

c. \#Kim broke the stick across the room.

d. \#Kim broke the stick purple.

e. \#Kim broke the stick into the ground.

f. *Kim broke her knuckles sore. (by breaking the glass with her fist)

Note that there is nothing pragmatically implausible about, say, $(14 b, c)$ or $(15 c-f)$ that might independently explain these data. There could be situations in which one clears a room by dimming the lights until everyone leaves, or that dimming the light strained Kim's eyes to the point of soreness. Similarly in $(15 c, e)$ breaking something might well cause it to move, in (15d) breaking a stick of a certain sort of wood might cause the color to change, and in (15f) breaking things with one's fist might make one's knuckles sore. But these situations cannot be described by $(14 \mathrm{~b}, \mathrm{c})$ or (15c-f). Thus there is something about canonical manner and result verbs that causes them to pattern differently on this diagnostic.

Of course, a question arises of why this would be the case. The simplest explanation is that if a result XP predicates a scalar result of some patient its scale must be compatible with whatever scale is entailed by the verb regarding scalar

${ }^{7}$ A reviewer notes that such examples may be acceptable in other languages, citing Mandarin as a possibility. We agree that it is an open question whether this test generalizes across languages, but focus on English here.

${ }^{8}$ Although Rappaport Hovav and Levin and Rappaport Hovav do not discuss this, there do appear to be a limited set of cases where result verbs indicating damage allow non-subcategorized objects in the context of result XPs indicating separation, as with John broke the branch off, where the branch comes off rather than being broken per se. However, this does require the existence of a larger entity which is now broken as a result, and the direct object must be a subpart of this entity. Otherwise, nonsubcategorized objects in general are not possible. 
change for the same patient (following Wechsler 2005a; Beavers 2008; see Beavers and Koontz-Garboden 2017a: 868-70 for a more detailed discussion of exactly this point, rooted in the Unique Path Constraint of Goldberg 1995: 81-8). This would rule out cases like $(14 \mathrm{~b})$ and $(15 \mathrm{c}, \mathrm{d})$, where the two scales are not compatible, and rule in cases like $(14 a)$ and $(15 a, b)$ where they are, as well as cases like (13a) where the verb presumably contributes no scale that the result XP could be incompatible with.

This does not explain the difference between manner and result verbs in allowing non-subcategorized object result XPs, though. However, with transitive result verbs the patient is almost universally the object (though see Verspoor 1997; Wechsler 1997 for counterexamples involve patient-subject/path-object motion verbs). Generating non-subcategorized object resultative constructions would first involve dropping that object, but being result verbs these verbs generally do not permit object drop, as discussed in \$4.2.2. This would thus rule out canonical transitive result verbs from appearing in these constructions, though since canonical manner verbs do allow object drop they should readily appear in this construction, explaining all of the data above. (We thank an anonymous reviewer for commentary on this point.)

That the relevant condition does hinge on the deletability of the object follows from a point made in Beavers and Koontz-Garboden (2017a: 851), where we note that non-subcategorized object resultatives are possible for intransitive scalar change verbs such as climb as in Moving so quickly on the rope that it cut his skin, John climbed his hands bloody, which is acceptable to some speakers. The explanation we posited is that in this case the two changes (movement for the subject of climb and soreness for the object) are predicated of different participants and so no scalar incompatibility arises. Key, though, is that there exists an intransitive variant of climb that is not subject to the ban on deleting objects since it does not take one anyway, freeing up the object position for the non-subcategorized object.

That said, Beavers (2011b: 360-1) notes (in contrast to the stronger statement from Rappaport Hovav above) that among result verbs there is some variability in how many result XPs are possible. For example, cool allows only degrees of temperature while $c u t$ allows a range of outcomes, including degrees of being cut up but also shapes (e.g. John cut the paper into an animal shape), i.e. things that are not cuttedness states per se but follow from or are connected to them. Beavers also suggests that certain manner verbs-surface contact and impact verbs such as hit and kick-also entail latent scales that limit result XPs (building on Tenny 1992: 20, (42); see also Simpson 1983: 151), although the constraints are rather loose. Similarly, Beavers and Koontz-Garboden (2017a: 869-70) demonstrate that certain kinds of manners may more readily license certain kinds of result XPs than others. In particular, we argue that verbs like drag that entail manners of motion that involve friction between the moving patient and the surface it moves along and 
thus more opportunities for changes arising by virtue of the nature of the manner allow more types of result XPs than verbs like slide that entail manners that require less friction (e.g. Sandy dragged/??slid the logs smooth).

However, none of these subtle details alter the larger point that result verbs as a class tend to have more limited result XP possibilities than manner verbs, and in some cases the constraints are fairly rigid. Having now outlined three diagnostics for result in the meaning of the verb, we now turn to tests for manner in the meaning of a verb.

\subsection{Manner entailments in verbs}

To identify manner in a verb's meaning we first need a definition of manner. To stay as close to Rappaport Hovav and Levin as possible, we adopt their definition of manner as non-scalar (i.e. nonmeasurable) change (Rappaport Hovav and Levin, p. 32):

"A non-scalar change is any change that cannot be characterized in terms of an ordered set of values of a single attribute... The vast majority of non-scalar changes deviate from scalar changes in another, more significant respect: they involve complex changes - that is, a combination of multiple changes-and this complexity means that there is no single, privileged scale of change."

Thus a manner is a complex sequence of separate changes that collectively define an action, but do not necessarily add up to a single cumulative change along any one dimension. Furthermore, something not made explicit by Rappaport Hovav and Levin, these changes are presumably also only temporary, in that there is no entailment that any subset of them holds at and/or persists beyond the end of the event. Otherwise, it would be difficult to defend the position that they do not constitute a result. Perhaps a paragon example of non-scalar change is the movement of arms and legs during running: the various movements of the limbs do not add up to any one particular change along any one specific scale. Thus manner and result can be seen as forming a dichotomy of types of changes-nonscalar change vs. scalar change (or non-scalar change vs. scalar result in the case of the verbs discussed here).

However, while this definition of result admits a relatively coherent unifying factor-change along a scale-it is not clear what single positive diagnostic unifies all manners on this definition, which presumably include not just types of physical motion, but also ways of speaking, making noise, emitting light, and even ways of sitting still. Neither Rappaport Hovav and Levin nor Rappaport Hovav (2008) address this issue, and it points to the need for further, explicit work on what 
exactly manner is. ${ }^{9}$ Nonetheless, for consistency, we stick to their definition as is, which is sharp enough to have some empirical consequences.

The challenge is in finding diagnostics for manner given the heterogeneity of non-scalar change. Rappaport Hovav (2008: 23) proposes that manner verbs can be picked out by acceptability with object deletion: since result verbs disallow object deletion, manner verbs must allow it (see \$4.2.2). However, this is an erroneous conclusion. First, object deletion is supposedly licensed by not entailing scalar change, which is not the same as entailing non-scalar change. In other words it is not positively associated with manner. Second, taking object drop also as a manner diagnostic means that it can only pick out manner in the absence of result; a verb that entails both should not permit object deletion by virtue of entailing result, despite also entailing manner. In other words, this test as originally formulated presupposes a binary contrast between manner and result verbs, but we are interested in a three-way contrast between result verbs, manner verbs, and manner+result verbs. Thus if we accept obligatory objects as a positive result diagnostic, as we do, we need separate diagnostics for manner.

Given the heterogeneity of manners, we are skeptical of what positive criteria there might be. For this reason we instead take on a more modest goal. To identify the existence of manner+result verbs, it is sufficient to isolate just one type of manner, and if a verb entails this as well as a result, then we can conclude that manner+result verbs exist. We therefore focus on intuitively the most canonical type of manner, namely moving various parts of the human body in exhibiting a certain action (e.g. in forming some physical pattern or manipulating an instrument), which for convenience we call being an "actor." This implies change (i.e. movement), but it is not scalar since it may involve sequences of unrelated movements and no lasting effect. We discuss three diagnostics for manner qua actorhood below.

\subsubsection{Manner diagnostic \# 1: Selectional restrictions}

First, returning to a point first made in $\$ 2.5 .1$ (see also $\$ 1.6 .2 .1$ ), if a transitive verb has actorhood in its meaning, then it should impose selectional restrictions on its subject. Pure result verbs can also have restrictions on their subjects, but they impose fewer than transitive manner verbs, permitting inanimates and natural

\footnotetext{
${ }^{9}$ Another challenge is that if change is non-scalar because it represents a confluence of various other changes, some individual changes in that collection may themselves be scalar (e.g. the motion of one particular part of a body that follows a particular path). Yet somehow this is not sufficient to define a verb entailing this non-scalar change as a scalar change verb. Rather, taking the definition of nonscalar change seriously, it must be that somehow the entire package of changes is deemed somehow unanalyzable. How to formalize this we leave aside here.
} 
forces as well as animates. Manner verbs describing actions, by contrast, generally do not permit natural forces or inanimates (with the exception, in the latter case, of inanimates that are interpretable as animate, as with certain machines, or as instruments under the control of some implicit agent, as with a reviewer's I like how this mop scrubs the floor; see also $\$ 1.1, \mathrm{fn} .2$ ). This contrast between result and manner verbs follows if result but not manner verbs require no specific action of their subjects. ${ }^{10}$ These manner verbs, by contrast, do require a specific action, which can be performed only by certain kinds of individuals, leading to contrasts in selectional restrictions between verbs lacking manner entailments and those having them, with the former allowing a wider range of subjects than the latter:

(16) a. John broke/shattered the vase with a hammer.

b. The hammer broke/shattered the vase.

c. The earthquake broke/shattered the vase.

(17) a. John scrubbed/wiped the floor with a stiff brush.

b. \#The stiff brush scrubbed/wiped the floor.

c. \#The earthquake scrubbed/wiped the floor. ${ }^{11}$

This test has also been proposed by Van Valin and Wilkins (1996: 310) for identifying agentivity in a verb's meaning, a notion presumably related to manner, and in the causative/inchoative alternation literature as determinant of which causatives have inchoative forms, namely those that impose no conditions on their subjects other than causation (as per $\$ 2.2 .1$; cf. The rope snapped/*cut; Guerssel et al. 1985; Hale and Keyser 1987; Haspelmath 1993; Van Voorst 1995; Reinhart 1996, 2002; Levin and Rappaport Hovav 1995: 99; Koontz-Garboden 2009a, inter alia, though see $\$ 2.5 .1, \mathrm{fn} .30$ on the inapplicability of causative/inchoative alternations as a manner verb diagnostic). ${ }^{12}$

\footnotetext{
${ }^{10}$ For simplicity, we restrict this test to transitive result verbs, where we assume that selectional restrictions relating to manner will be imposed on the subject indicating the type of action it performs, though in principle such restrictions could also be imposed on the object (see e.g. the event structures of Rappaport Hovav and Levin 1998: 113). As Fillmore (1970: 128-9) notes, patienthood may also involve selectional restrictions, but for transitive result verbs these will almost always be localized to object position and thus should have no effect on the choice of subject. However, this test cannot distinguish intransitive result and manner verbs, since all selectional restrictions of any sort will (of necessity) be imposed on their single arguments.

${ }^{11}$ A reviewer suggests that $(17 \mathrm{c})$ might be independently disallowed due to a more general constraint that lexical causatives entail direct causation as per $\$ 1.6 .2 .1$, where an earthquake cannot directly cause scrubbing or wiping without some intervening chain of events. We disagree-a plausible direct causation scenario would be if a towel was on the floor and the earthquake caused it to move around, thereby scrubbing/wiping the floor. In this case, it is still odd to say (17c). Rather, wipe/scrub involve a particular method of applying the instrument to the patient.

12 A reviewer does wonder if result verbs may place some constraints on their subjects nonetheless, giving for example ???John's right arm/hand broke the vase (the judgment is the reviewer's). We do not find this example unacceptable, and attested cases are not difficult to find (cf. Finally, on the fourth try, his left arm broke the glass; <https://www.sun-sentinel.com/news/fl-xpm-1995-07-01-9507010108-
} 


\subsubsection{Manner diagnostic \# 2: Denial of action}

A more direct way to diagnose actorhood would be to look for inferences that follow from verbs entailing it, as we first did in $\$ 2.5 .1$. We could look at verbspecific inferences, as we did in $\$ 4.2 .1$ with past participles of specific result verbs. For example, with jog or blink it is straightforward to isolate particular manners that cannot be denied in each case (e.g. \#John ran, but did not move his arms and legs or \#John blinked, but did not move his eyelids). Conversely, with break or destroy it is harder to isolate such an action (e.g. John broke/destroyed the vase, but didn't move his arm/drop it off a ledge/kick it with his foot/etc.). However, like the past participle test, this approach is potentially open to the criticism that we have not shown that the relevant non-cancelable inferences for each verb constitute the same notion of manner.

So we might look for some single, non-verb-specific inference that holds for all manner verbs entailing actorhood, in other words the manner equivalent of something is different about $x$. In particular, if someone is an actor, it should be impossible to assert that they performed the action specified by the verb and yet didn't move a muscle. As expected, prototypical actor-oriented manner verbs, such as manner of motion verbs as in (18), are contradictory in this frame, showing that they entail actor-oriented manner:

\section{(18) \#Jim ran/jogged/blinked, but didn't move a muscle.}

By contrast, if all result verbs entail is a result but not (any specific type of) action, then it should be possible to deny that action occurred. But, focusing again on manner of causation, how can one cause something without acting in some way? As per $\$ 1$ 1.6.2.1 it is not our goal to outline a general theory of causation, but an example might be negligence-failing to act in some (expected) way to prevent a change from occurring, thereby being responsible for it. In fact, exactly such a type of causation is subsumed under the force-dynamic model of causation of Talmy $(1976,1988,2000: 471-550)$, later expanded on by Wolff $(2003,2007)$, Wolff and Song (2003), and Wolff et al. (2010), who propose a typology of causation types that invoke not just a notion of causation by action (CAUSE), but also aiding and letting (ENABLE) for cases where a change is in progress and the "enabler" does not stop it and/or aids it in its progress. Negligence falls under an ENABLEtype causation that Talmy (2000: 420-1) calls "extended letting" (giving The plug's staying loose let the water drain from the tank), wherein some causer that could stop some patient from changing nonetheless remains disengaged from the ongoing

story.html> Accessed September 30, 2019). Nonetheless, as noted above, it may be that some verbs previously classified as result verbs do have manner components, at least for some speakers. 
change. ${ }^{13}$ Of course, not every failure to prevent something is causation-as Talmy (2000: 477) points out, I emptied the tank cannot be true if the speaker was merely present but doing something totally unrelated to the tank. However, if there is some specific possibility or expectation of preventing a change, that participant could conceivably be said to have caused the change by failing to stop it.

With this in mind, if result verbs entail causation but not actorhood per se, then they should in principle be compatible with didn't move a muscle in a negligence context, even if other prerequisites for actorhood (e.g. being animate or human) obtain. This is possible, as shown in the following context with destroy, where the owner of a car can be said to have destroyed the car through (deliberate or nondeliberate) negligence, a type of non-action (furthermore a type of causal state as per Beavers and Zubair 2013: 32-6): ${ }^{14}$

(19) Jim destroyed his car, but didn't move a muscle-rather, after he bought it he just let it sit on his neighbor's lawn on cinder blocks, untouched, until it disintegrated!

Thus, at least some result verbs do not in fact actually entail actorhood.

However, as a reviewer points out, some supposed result verbs seem to resist this frame, including in particular break. For example, (20) seems at first to be a contradiction:

(20) \#Kim broke my DVD player, but didn't move a muscle.

Yet, although animate causers are typically actors in breaking events, this is not always the case. This is shown by placing (20) in the negligence context in (21), which mitigates the apparent contradiction:

(21) Kim broke my DVD player, but didn't move a muscle-rather, when I let her borrow it a disc was spinning in it, and she just let it run until the rotor gave out!

13 This in turn corresponds to what Wolff and Song (2003: 44-5) call FAIL TO PREVENT and Wolff (2007: 88) calls ENABLE. (See also Kehler 2002: 21 on "denial of preventer.") Most particularly, in an experimental study Wolff et al. (2010) outline and motivate the linguistic relevance of various types of "causation by omission," including cases where something prevents itself from preventing something (pp. 205-10), although their particular scenarios involve an active attempt at preventing that is suddenly stopped short, rather than wholesale failing to act.

${ }^{14}$ This reading of destroy relies on the expectation that the causer would or should have acted to stop the change, but failed to. One could instead say that result verbs do entail an (unspecified) action, but unlike manner verbs, it need not obtain in the real world (e.g. it is embedded under a sublexical modality à la Koenig and Davis 2001, or is a "virtual force" à la Wolff, Barbey, and Hausknecht 2010: 206). In this case, there is still a distinction between manner and result verbs-the former require action in the real world, the latter do not. 
Thus at least some canonical result verbs allow readings where the causer is not an actor. The reason (20) seems infelicitous is that, absent an appropriate negligence context, the default reading for result verbs with human subjects is that an action was performed. But this is not part of the verb's meaning, and is instead derived pragmatically, in line with the pragmatic principle recognized by Holisky (1987: 118-9) for interpreting human causers as agents in the absence of contrary evidence (see also Van Valin and Wilkins 1996). Additionally, a reviewer notes that subject choice may have an effect, giving \#The army destroyed the city, but didn't move a muscle in contrast to (19), where necessary actorhood may be a contingent fact about armies destroying cities. However, given that negligence is possible with other uses of destroy, destroy does not itself require actorhood. Conversely, no context will change the outcome of this diagnostic with pure actororiented manner verbs; in these cases it must be the verb itself that determines the behavior in this diagnostic.

\subsubsection{Manner diagnostic \# 3: Complexity of action}

A third diagnostic relates to the complexity of the action described by the verb. Rappaport Hovav and Levin (p. 32) suggest that "the vast majority" of manners are complex, consisting of a series of separate changes. Dowty (1979: 170-1) further notes that complex manner verbs like waltz require nontrivial time intervals to evaluate (i.e. more than two moments; waltzing requires at least three steps). Thus complex manner verbs should be durative, as outlined in $\$ 1.6 .2 .2$, and this can serve as a diagnostic. However, as discussed in \$1.6.1.1 (see also \$3.7) durativity is also contingent on the nature of the scale underlying the change, where among canonical result verbs non-gradable (two point) scalar changes in general yield punctual predicates and gradable scalar changes yield durative predicates. Thus both manner and result can have an effect on the durativity of the overall verbal predicate. So what if a verb entails both manner and result?

Assuming both contributing factors are active at once, the effects of one would mask the effects of the other. A punctual predicate could either entail just a simple manner or just a two-point change, or both, but nothing about its punctuality indicates which. A durative predicate allows more possibilities: it may entail either a complex manner (where the change can be non-gradable, gradable, or nonexistent), or a gradable change (where the manner can be simple, complex, or non-existent). But durativity does not tell us which it is. However, if we know independently that the verb entails non-gradable scalar change, so that the scale has only two points, then if the predicate is durative, it must be because there is a complex manner. So the durativity test can be useful for identifying manner in a verb's meaning, provided we control for certain mitigating factors. Having now 
outlined three result tests and three manner tests, the question is whether any verb will pass both sets of tests, which we turn to now.

\subsection{Classes of manner+result verbs}

With the above tests in place, we now turn to the question at hand, namely the existence of verbs that entail both a manner and a result at the same time. The verbs we are interested in here are in fact a subset of the verbs discussed in the preceding chapters. In Chapter 2 we discussed verbs of change of state that seemed to have change as part of their root meanings. We will show here that a subset of these, namely verbs of manner of cooking (e.g. sauté, braise) (Levin 1993: 243) and verbs of manner of killing (e.g. guillotine, drown) (Levin 1993: 230-3, Krohn 2008) entail not just a change but also a manner at the same time, i.e. a particular action that caused the change. We will also discuss ditransitive ballistic motion verbs (e.g. throw, toss) (Gropen et al. 1989: 243-4), a class we discussed in Chapter 3 and in fact analyzed as entailing both a manner and a result, though we did not fully justify that claim. ${ }^{15}$

The key empirical argument is that, as we show, in all cases these verbs pattern as distinct from canonical result verbs and canonical manner verbs in that they pass both sets of tests, i.e. they pattern like result verbs on result tests and manner verbs on manner tests. This suggests that they have both components as a part of their meaning, thus falsifying Manner/Result Complementarity at least as a claim about the truth conditional content of particular surface verbs. This does not, however, address whether Manner/Result Complementarity might hold for these verbs in some structural sense of their event structures only having either a root adjoined to $v_{\text {act }}$ or $v_{\text {cause }}$ presumably describing a manner or a root complement to a $v_{\text {become }}$ head presumably describing a result state but not both simultaneously. ${ }^{16}$ We address this question in $\$ 4.5$.

\footnotetext{
${ }^{15}$ Rappaport Hovav (2015: 5-6) suggests that the claim of complementarity is restricted to monomorphemic verbs, so that lack of complementarity can, in principle, arise in a morphologically complex verb as a consequence of templatic meaning, compounding, etc. In such a case, the claim is that the two classes of inferences are coming from separate places. First and foremost, in at least some versions of event structures all verbs are non-monomorphemic, as mentioned in $\$ 4.1$ for syntactified approaches that require both roots and templatic heads. Thus Rappaport Hovav's claim must perhaps be revised to be about some type of surface monomorphemicness, or perhaps just a root plus only certain types of heads and not others (e.g. a root and one type of categorizing head). However defined, it could be that some of the verbs we examine are not monomorphemic in the appropriate sense. as with guillotine, which has zero-related nouns, and might well be denominal. What exactly the direction of derivation is is unclear in English, but we believe it is in fact irrelevant to the evaluation of the complementarity claim, given that the restitutive modification facts discussed further below show unambiguously that the manner and result inferences lie in the root, and do not come from any templatic structure.

${ }^{16}$ We say that the roots "presumably" entail a manner or result in the appropriate position since technically it is possible that a root in one or the other position does not have the canonical semantics we would expect, something we return to in $\$ 5.4$.
} 
Before proceeding with the truth conditional argument, though, we briefly motivate the need for such an extensive discussion of this question. It may seem obvious that a verb like braise or guillotine gives rise to non-cancelable inferences of both a result and a means of achieving it. However, as Rappaport Hovav and Levin's proposal makes clear this is not necessarily so. Perhaps only manner or result is entailed, and the other is inferred through some process of conventionalization (the stance of e.g. Levin and Rappaport Hovav 2008). Or perhaps braise and guillotine are polysemous between manner and result uses, giving rise to the impression that it entails both (as both a reviewer and Beth Levin, p.c., suggest; see Levin and Rappaport Hovav 2013 for discussion of this type of analysis for climb and cut and Beavers and Koontz-Garboden 2017a for a counterargument regarding climb). Alternatively, braise and guillotine could entail both meaning components, but they are different from those found in canonical manner and result verbs. If event structures constrain non-cancelable verbal meanings and are constructed in ways that predict complementarity between two specific meaning components, it is an important question whether the meanings associated with supposed manner+result verbs really are the same as those in canonical manner verbs and those in canonical result verbs, and thus we devote some space to this question. We consider the various alternative analyses again in \$4.4.4.

\subsubsection{Manner of killing verbs}

Among the verb classes in Levin's (1993) encyclopedic classification are "verbs of killing" (Levin 1993: 230-3), which she divides into two subclasses. First are murder verbs, which "all describe killing... None of the verbs in this class lexicalizes a means component; that is, none provides any information about how the killing came about" (p.231) (a reviewer doubts immolate belongs here; we concur, but leave it because it is in Levin's list):

(22) Levin's murder verbs: assassinate, butcher, dispatch, eliminate, execute, immolate, kill, liquidate, massacre, murder, slaughter, slay

Second, poison verbs "relate to actions which can be ways of killing. Thus they each lexicalize a means component, and it is this means which differentiates among them" (p.232):

(23) Levin's poison verbs: asphyxiate, crucify, drown, electrocute, garrote, hang, knife, poison, shoot, smother, stab, strangle, suffocate

Levin herself observes that her initial observations and classification may oversimplify things, particularly for poison verbs, remarking that "In principle, as means 
verbs, these verbs need not entail that the action they denote results in death; however, some of them do appear to have this entailment" (Levin 1993: 232). We demonstrate that at least some poison verbs do indeed entail both manner and result, namely death or a state that can lead to death. We call these manner of killing verbs, which include those in (24): ${ }^{17}$

(24) Manner of killing verbs: crucify, drown, hang, guillotine, electrocute

To demonstrate this, we show that these verbs pass all of the result tests and all of the manner tests.

Starting with result tests, (25) show that these verbs generate a contradiction in the result entailing frame, patterning with canonical result verbs:

(25) a. \#Jane just drowned Joe, but nothing is different about him.

b. \#Jane just hanged Joe, but nothing is different about him.

c. \#Jane just crucified Joe, but nothing is different about him.

Second, like result verbs, manner of killing verbs disallow object deletion:

a. *All last night, Shane crucified.

b. *All last night, the executioner electrocuted.

c. *All last night, Shane drowned.

(on intended reading)

Third, manner of killing verbs pattern like result verbs in terms of the result XP diagnostic, in that the result XPs they occur with are fairly restricted. While they can take result XPs specifying (redundantly) death, as in (27), they cannot take result XPs that specify other result states, as in (28), despite the pragmatic plausibility of these sorts of manner+result combinations:

(27) a. Faulty ground wires in a building electrocuted him to death in 2004. $<$ http://buzz.yahoo.com/article/1:y_news:31f4c8213ef1e2e4e5ae60d75a 00b97f $>$ (Accessed 2010)

b. An electricity bolt came from the roof of the computer's self-defence unit that electrocuted him dead. (Thurlow, Trevor Lionel. 2015. No Future Without War, Xlibris, ISBN 978-1-5035-0833-0; no page numbers provided.)

${ }^{17}$ Jespersen (1927: 335) first notes that "Drown is generally used in the transitive sense 'to kill by placing under water', but the participle drowning is often, and the other forms sometimes, found in the intransitive sense 'to die under water', 'to get drowned'." Dowty (1979: 203-4) similarly notes in passing that drown and electrocute have the meaning of kill with a specific manner filled in. 
(28) a. \#Shane electrocuted the prisoner to a crisp.

b. \#Shane drowned Sandy blue.

c. \#Shane hanged the prisoner thin.

d. \#The Romans crucified Jesus to the tomb.

Likewise, manner of killing verbs resist non-subcategorized object result XPs: ${ }^{18}$

(29) a. \#Shane electrocuted the handle right off the wall.

b. \#Shane drowned Sandy's lungs totally full of water.

c. \#Shane hanged the noose off the gallows.

d. \#The Romans crucified the cross in half.

Thus manner of killing verbs seem to have a result component.

At this point we reiterate of course a point made in $\$ 4.1$ that while our intuitions are that the result is death, this may not hold for all speakers, though it is uncontroversial that some result obtains even if not death, e.g. crucify at least entails a change of location qua being put into a particular spatial configuration, while guillotine entails some injury if not death (see fn. 18). As noted in $\$ 4.1$ one place where we have found more radically different speaker judgments is with electrocute, where for many speakers death is an entailment but for some the meaning is essentially that of shock and there is no result at all. For these speakers the verb is simply lexicalized as a manner verb. Indeed, a reviewer who judges electrocute to mean shock (i.e. to be a manner verb) finds electrocute acceptable with nondeath result XPs, giving the (naturally occurring) Spider-Man has to fight Rhino in a generator room, defeating him by making him smash into six generators that electrocute him into submission <http://en.wikipedia.org/wiki/Rhino_(comics)>. For such speakers this is the expected behavior for a standard manner verb. Another reviewer finds electrocute to a crisp acceptable, though this may again be a shock reading. But this verb being a manner verb for some speakers does not undercut the point that for some speakers it entails a result.

Turning to manner diagnostics, manner of killing verbs, like canonical manner verbs, disallow inanimate and natural force subjects:

(30) a. John hanged/crucified Jesus with sailing rope.

b. \#Sailing rope hanged/crucified Jesus.

c. \#The wind hanged/crucified Jesus (by opening the trap door/raising his cross).

\footnotetext{
${ }^{18}$ As a reviewer notes, it is possible to say The executioner guillotined the man's arm off, demonstrating the same sort of part/subpart non-subcategorized object property seen with break off in fn. 8 is possible with these verbs as well. This in fact further supports the claim that manner of killing verbs are like result verbs.
} 
(31) a. The revolutionaries guillotined the queen with a rusty blade.

b. \#The rusty blade guillotined the queen. ${ }^{19}$

c. \#The heavy wind guillotined the queen (by loosening the blade).

Thus manner of killing verbs place restrictions on their subjects, just like canonical manner verbs.

Second, manner of killing verbs pattern with manner verbs in that it is contradictory to assert that the subject killed the patient with one of these verbs and to deny any action was performed, even in the sorts of contexts, like those above, where causation is entailed but actorhood is not. This is illustrated by (32), where, to our ears, one cannot be accused of electrocuting, hanging, drowning, or crucifying someone simply by negligently failing to prevent it, in contrast with the canonical result verbs as discussed in $\$ 4.3 .2$ :

(32) a. \#The governor electrocuted/crucified the prisoner, but didn't move a muscle-rather, after taking office she failed to issue a pardon!

b. \#The governor drowned/hanged the prisoner, but didn't move a musclerather, during the execution she just sat there, tacitly refusing to order a halt!

This is not to say that the governor in (32) could not be held responsible for the prisoner's death. But it is anomalous to express this with a manner of killing verb. Thus these verbs entail that their causers have actor-oriented manner, and are therefore manner-entailing.

The third test has to do with the effects of the manner on durativity. Here, as we noted above, it is important to first be sure there is not gradable change in the meaning of the verb, since this would make the predicate durative regardless of the presence or absence of a manner component. Using gradability of the deverbal adjective as a diagnostic, (33) shows that the corresponding adjectives tend to resist comparative morphology and thus manner of killing verbs likely entail change along a two-point scale, in other words they describe non-gradable scalar change:

\#more hung, \#more drowned, \#more electrocuted, \#more crucified.

This is not surprising: these verbs likely entail death, and dying is generally viewed as non-gradable change (cp. \#more dead/killed). However, by virtue of the nongradable change, they should be punctual. If they are not, then it must be because

${ }^{19}$ A reviewer finds this acceptable, though this may be a case of an instrument subject reading with an implicit agent; see $\$ 4.3 .1$ and also $\$ 1.1$, fn. 2 . 
of a manner component. In fact, many manner of killing verbs are durative, as in (34), where in an hour allows both an after and a during reading:

(34) I can drown/hang/crucify Jim in five minutes ...

a. because I lack the courage.

(after five minutes)

b. because that is how long it takes to kill someone by holding them under water/cutting off their air/nailing them down to a cross, hoisting them up, and waiting.

(during five minutes)

Thus again (at least some) manner of killing verbs have a manner component. A few manner of killing verbs are punctual, as in the following, which could involve a beheading via one quick slice:

I can guillotine Jim in five minutes (with one slice).

(after five minutes)

This does not show that guillotine lacks manner, only that it is not a complex manner. But for durative manner of killing verbs that entail non-gradable change, this test does suggest that they entail manner. In sum, manner of killing verbs appear to violate Manner/Result Complementarity as a truth conditional claim about possible verb meaning, since they have both a result and a manner component at the same time.

Finally, a reviewer suggests that if we look at murder verbs we might find a set of patterns that calls the results of the manner diagnostics for the poison verbs into question. In particular, at least some murder verbs-including murder and assassinate-place selectional restrictions on their subjects owing to an intentionality condition (e.g. \#The hurricane murdered/assassinated the senator), but perhaps not any specific action per se (and thus didn't move a muscle may not apply, as in a gangster ordering a hit). This might suggest that the subject selection tests does not always pick out the same property of verbs, calling it into question as a manner test. However, our point here was not to have a single set of diagnostics that work for all types of things one might call manner, nor was it to have a test that uniquely and always picks out one subtype of manner. Rather, the goal was to test for a specific type of manner, namely human action, which will generate subject selection effects, among other things, so as to identify at least some subclass of verbs that do entail both manner and result at the same time. Although it is true that intentionality can yield similar restrictions, there is no intentionality for poison verbs since they accept both deliberately and accidentally as modifiers (e.g. The executioner deliberately/accidentally guillotined the prisoner) whereas murder and assassinate do not (e.g. in The hitman ?deliberately/\#accidentally murdered/ assassinated the senator the modifier deliberately sounds slightly redundant and accidentally is out), leaving actorhood as the only obvious explanation. (That 
said, see Jimenez 2018 on intentionality in murder verbs and its relationship to Manner/Result Complementarity.)

\subsubsection{Manner of cooking verbs}

There are also verbs in Levin's (1993: 243) cooking class in (36) that entail both manner and result, as also noted by Goldberg (2010: 49-50). Many of these verbs describe manners of bringing about the result that a particular foodstuff go from raw to cooked (in some fashion or to some degree):

(36) barbecue, blanch, braise, broil, deep-fry, fry, grill, hardboil, microwave, poach, roast, sauté, stew, toast...

We focus on poach, sauté, and braise. First we consider result diagnostics. As (37) show, denying that the object changed yields a contradiction:

(37) a. \#Shane just poached the egg, but nothing is different about it.

b. \#Shane just sautéed the onions, but nothing is different about them.

c. \#Shane just braised the chicken, but nothing is different about it.

Secondly, these verbs disallow object drop, as shown in (38):

(38) a. *All last night, Shane poached.

b. ??All last night, Shane sautéed.

c. *All last night, Shane braised.

Furthermore, these verbs disallow result XPs that are not a further specification of the result entailed by the verb, here a state of cookedness, as in (39):

(39) a. \#Shane poached the egg in half.

b. \#Shane sauteed the onions straight.

c. \#Shane braised the duck to the back of the oven.

(e.g. if the temperature was so hot it caused the pan to move)

A reviewer finds some such examples possible, giving Ming stewed the beef tendon stiff/soft/to pieces/black and You can poach these vegetables green, even though they are pale yellow when raw (our judgments are that the latter is unacceptable and the former unacceptable with black). However, as noted in $\$ 4.2 .3$, some result verbs may allow result XPs that are more loosely connected to the verbal result (here cookedness, though this may encompass textures and colors). What matters 
most is that the possible result XPs are somewhat constrained. Finally, result XPs predicated of non-subcategorized objects are unacceptable:

(40) a. * Shane poached the water bacteria-free. (e.g. while poaching an egg)

b. * Shane sautéed the seasoning off the pan. ${ }^{20}$ (e.g. while sautéing onions)

c. * Shane braised the pan dry.

(e.g. while braising duck)

Thus these verbs clearly entail a result, which seems correct given their intuitive meaning.

Turning to manner, the durativity diagnostic is unfortunately hard to apply. As discussed above, this diagnostic relies on the verb entailing a non-gradable scalar change, and indeed Levin (2008) suggests that this is true for at least sautée and poach, given the following sentences with her judgments:

(41) a. \#This egg is more poached than that one.

b. \#These vegetables are more sautéed than those.

(Levin 2008: 15)

However, we find these data relatively acceptable, and naturally attested examples can be found:

(42) a. When everything is cooked to your liking (some like they're [sic] mushrooms more sautéed than others." [sic] Add pesto, and cook just until pesto is warmed. <http://diymeraki.com/pesto-mushroom-arugula-salad/> (Accessed June 15 2019)

b. My eggs were more poached than the ones they served at the Café Hon under hollandaise. No, this was just a reminder that the tap on the juices of youth hadn't... (Devens, Toby. 2013. Happy Any Day Now, New York: NAL Accent, Penguin Group).

Furthermore, although more may be unacceptable to some people or less common with these adjectives, lightly or slightly, which also convey multiple degrees, are not difficult to find examples for, and such modifiers are generally not acceptable with non-gradable adjectives (cp. \#(s)lightly dead/pregnant):

(43) a. Eating lightly sauteed cabbage and raw granola cereal at $1 \mathrm{am}$. Am I pregnant or what? LOL <http://www.twitter.com/KristensRaw/status/ 7361004218> (Accessed May 8 2018)

${ }^{20} \mathrm{~A}$ reviewer finds this sentence acceptable. We disagree and note in (weak) support of our judgments the fact that there are no hits in Google for either "sautéed the seasoning off" or "sautéed the teflon off" (by contrast with "burned the seasoning off" and "burned the teflon off?" for which there are indeed examples). 
b. Chard can also be lightly braised in stock or warm water. <http://www. wisegeek.com/what-are-some-different-ways-to-cook-chard.htm $>$ (Accessed May 8 2018)

Thus these verbs entail gradable change (also the conclusion of Goldberg 2010: 50). They are therefore expected to be durative (cp. He sautéed/poached the ham in ten minutes allows a durational reading), obscuring any effect of manner. ${ }^{21}$ Nonetheless, our other manner tests suggest that these verbs do entail a manner, since the infelicity of (44) shows that it cannot at once be asserted that someone is cooking using one of these verbs while denying that they are moving:

(44) a. \#Shane poached the egg, but didn't move a muscle.

b. \#Shane sautéed the onions, but didn't move a muscle.

c. \#Shane braised the chicken, but didn't move a muscle.

These examples are not saved by a negligence context like the ones in (45):

(45) a. [ A salmon fillet is in a pan waiting to be poached, and Shane is in charge of making sure nothing happens to it until dinner time. A faulty wire triggers the stovetop burner and the water in the pan starts to simmer anyway, cooking the salmon. ]

\#Shane poached the salmon, but didn't move a muscle-rather, he just sat there while the heat simmered the water.

b. [ Some chopped onions are sitting in a pan in oil waiting to be sautéed, and John is in charge of making sure nothing happens to them until dinner time. The stove top suddenly heats up owing to a faulty wire, cooking the onions. ]

\#Shane sautéed the onions, but didn't move a muscle-rather, he just sat there while the faulty stovetop fried them. ${ }^{22}$

c. [ A chicken is sitting in a pan of braising liquid inside an oven, and Shane is in charge of making sure nothing happens to it until dinner time. Owing to a faulty wire the oven heats up and the chicken ends up braising. ]

\#Shane braised the chicken, but didn't move a muscle-rather, he just sat there while faulty oven cooked it.

\footnotetext{
${ }^{21}$ Levin (2008) uses the observation that sauté entails non-gradable change as evidence against its entailing a result. However, as discussed in $\$ 1.6 .1 .1$ (see also $\$ 3.7, \$ 4.3 .3$, and $\$ 4.4 .1$ ), scalar changes, and hence results, can be non-gradable - this is the case for any achievement verb. As such, the conclusion that sauté does not entail a result on the basis of the inability of an adjective derived from it to take a gradable modifier is unwarranted. Rather, the more refined result diagnostics that we laid out above show that it does entail a result.

${ }^{22}$ Arguably (45) is unacceptable because becoming fried is not what constitutes sautéing, which typically and perhaps necessarily involves moving the food around for even cooking and to prevent burning, though this just further goes to suggest that there is a manner in this verb.
} 
To say one has poached, sautéed, or braised something requires direct involvement of the causer. Likewise, these verbs impose selectional restrictions barring inanimate subjects (save perhaps instruments controlled by an unexpressed agent; see $\$ 1.1$, fn. 2) and natural forces (though judgments vary):

(46) a. Shane poached/braised/sautéed the duck in a new Le Creuset pan.

b. ??Shane's Le Creuset pan poached/braised/sautéed the duck perfectly.

c. ??The heatwave/even heat poached/braised/sautéed the duck to perfection.

Thus these verbs are manner entailing as well as result entailing, and as such are yet another example of verbs that violate Manner/Result Complementarity as a truth conditional claim about possible verb meaning.

\subsubsection{Ditransitive ballistic motion verbs}

The final verb class we consider here are ditransitive verbs of caused possession. In Chapter 3 we had analyzed such verbs as having roots that contribute a manner component and act as adjuncts to $v_{\diamond \text { cause }}$, although we had also suggested that their roots also entail some kind of result, giving rise to so-called root telicity and root durativity effects. However, we did not fully justify that these verbs entail a manner as well as a result on the basis of any particular diagnostics. We address that here, taking the class of ballistic motion verbs from the classification of Gropen et al. (1989: 243-4) as our main case study, applying manner diagnostics while also shoring up our prior analysis that they entail results with the result diagnostics developed here:

\section{(47) Throw verbs: fling, flip, kick, lob, slap, shoot, throw, toss}

We focus in particular on throw, toss, and flip.

The fact that these verbs entail a change of location is relatively uncontroversial. We demonstrated this in $\$ 3.6 .2$ using verb (class)-specific entailment patterns, and Rappaport Hovav and Levin (2008: 135) themselves observe the same, noting that "...throw-type verbs entail change of location... [s] omething cannot be thrown... without changing its location." Unfortunately, because the restricted result XP and object drop result diagnostics of $\$ \$ 4.2 .2-4.2 .3$ both work only for transitive verbs, we cannot apply them directly to throw-type ditransitives. However, a reviewer does suggest that we could check the transitive variants of these verbs for these diagnostics. While it may be that the transitive variants are somehow derived from very different event structures or possibly different roots, it is worth noting that they fail to permit object drop:

(48) \#All last night, Kim threw/tossed/flipped.

(on the intended readings) 
They also do not permit result XPs not directly related to motion:

(49) \#Kim threw/tossed/flipped the ball to pieces.

Finally, they do not permit non-subcategorized object resultatives:

*Kim threw/tossed/flipped his arm sore.

This is all consistent with the transitive variants of ditransitive verbs of ballistic motion entailing result, as we claim to be the case for their corresponding ditransitive variants.

Returning to the fully ditransitive uses, the contradiction diagnostic of $\$ 4.2 .1$ at least is directly applicable, and shows once again that result is indeed entailed (the relevant entailment covering all of these verbs-and others of change-oflocation-is $x$ is somewhere else, as mentioned in \$4.2.1).

(51) a. \#Mary just threw John the ball, but it is not somewhere else.

b. \#Mary just tossed John the ball, but it is not somewhere else.

c. \#Mary just flipped John the empty can, but it is not somewhere else.

This supports the claim that these verbs entail a result, viz. a change of location. As discussed in \$3.6.2 the relevant change of location is that the agent releases the theme, and as such it ceases to be with the agent.

If these verbs all entail change of location how do they differ from one another? Rappaport Hovav and Levin themselves propose that what distinguishes them is in fact manner:

...throw-type verbs entail change of location but not change of possession... What distinguishes among such verbs is how the force is imparted; they have a manner root (e.g. lob, throw) or, perhaps, an instrument root (e.g. kick, shoot).

(Rappaport Hovav and Levin 2008: 135)

Unfortunately, the complexity diagnostic will not work since, as Beavers (2011a) shows and as discussed in $\$ 3.7$, nearly all of these verbs are punctual. The primary exception to this as discussed in the previous chapter was the verb ricochet, though the durativity seemed to come from the extended path and thus it will be impossible to use its durativity to tell whether it also entails a manner. However, these verbs do seem contradictory with didn't move a muscle, even in a context where the subject could plausibly be said to be a negligent causer as in (52): 
(52) [ A tennis coach sets up a ball machine that sends balls to the students, and puts Isaac in charge of the machine, telling him to stop it at 4:00pm. ]

??Isaac threw/tossed/flipped the kids the balls after 4:00pm, but didn't move a muscle-rather, he failed to stop the ball machine at the specified time.

Rather, to say one threw, flipped, or tossed someone a ball entails some action on the causer's part. Furthermore, throw-type verbs show subject selectional restrictions, dispreferring inanimates (save perhaps instrumental subjects reading where there is an unexpressed agent; see $\$ 1.1$, fn. 2 ) and natural forces: ${ }^{23}$

(53) a. John threw/tossed/flipped Sandy the ball with his new glove.

b. \#The new glove threw/tossed/flipped Sandy the ball.

c. \#The wind threw/tossed/flipped Sandy the ball.

Thus throw-type verbs entail both a result and an (actor-oriented) manner, consistent with Rappaport Hovav and Levin's (2008) claims, and thus are really manner+result verbs, exactly as proposed in Chapter 3 .

\subsubsection{Summary of manner+result verbs}

In this section we demonstrated that there are at least three classes of verbsmanner of killing verbs, cooking verbs, and ditransitive ballistic motion verbsthat entail both a manner and a result as part of their meaning. The existence of these verbs thus means that at least as a truth conditional claim about verb meaning, Manner/Result Complementarity, does not hold. In addition to canonical result verbs (which entail no manner) and canonical manner verbs (which entail no result) we have mixed classes that entail both. (The results in Beavers and Koontz-Garboden 2017a further add numerous manner of motion verbs to the list of manner+result verbs as well.)

However, as noted in $\$ 4.4 .1$, some alternative analyses present themselves, and we briefly discuss and dismiss these alternatives here. First, it could be that each verb discussed above only entails a manner or result, and whichever is not entailed arises through some conventional inference based on the specific entailed meaning as per Levin and Rappaport Hovav (2008). If this were so, the additional inference

${ }^{23}$ Some machines, such as pitching machines, are possible, consistent with the widespread observation that machines can behave like actors (see e.g. Cruse 1973: 16). As noted in $\$ 4.3 .1$ more broadly, judgments may vary depending on how one conceptualizes of the relevant subjects. 
should be defeasible; yet in the above tests it was not possible to just cancel away either meaning component.

Conversely, as both a reviewer and Beth Levin (p.c.) have pointed out, it could be that each verb is polysemous between a pure manner and a pure result reading (as Levin and Rappaport Hovav 2013 suggest for climb and cut). On this analysis, the reason they pass all tests for both manner and result is because the result use passes the result tests and the manner use passes the manner tests. Thus (54a) is infelicitous because this variant of guillotine is the result one, while (54b) is infelicitous because this variant of guillotine is the manner one, with neither variant entailing both at the same time:

(54) a. \#The peasants guillotined the queen, but nothing is different about her.

b. \#The heavy wind guillotined the queen (by releasing the blade).

However, if guillotine and the other verbs discussed above were polysemous in this way, it would actually predict the opposite pattern to (54). For example, in a neutral context as we have tended to use above, (54a) should admit a felicitous reading with guillotine interpreted as a manner verb, while (54b) should admit a felicitous reading with guillotine interpreted as a result verb. In other words, if these verbs were polysemous, they should fail all of the tests, rather than pass them. That this is not so suggests they are not polysemous, but instead entail both meanings at once. (See also Beavers and Koontz-Garboden 2017a, who show that Levin and Rappaport Hovav's argument regarding the polysemy of climb also does not hold up).

Alternatively, Husband (2011) suggests that while some of the verbs we claim to have manner+result entailments do seem to have inferences of manner and result in them simultaneously, the two inferences are not quite identical in their status. In particular, he claims that the result entailment is a presuppositional rather than part of the asserted content, whereas the manner entailment is part of the asserted content. He argues for this based on the empirical claim that the manner can be directly questioned in a polar interrogative but the result state cannot be:

\section{A: Was King Louis XVI guillotined?}

a. B: Yes, he was killed by a guillotine.

b. B: \#No, he didn't die.

(Husband 2011: 119, (16a), (17a))

Husband thus suggests (p. 120, (23)) that Manner/Result Complementarity holds at least as a fact about asserted content. Our own judgments are that (55b) is not an unacceptable response in the intended sense to the question, and similarly for other examples Husband gives, though of course there can be speaker variation on issues such as this. More importantly, while it is an interesting question exactly how the relevant meaning components are introduced (and for now we continue 
to assume for expository purposes that they are not presuppositional in nature) the larger point is that a single lexical item derives both inferences at once and this must be coming from its event structure given the logic of how event structural approaches work, something with which Husband (2011: 121) concurs. ${ }^{24}$ Thus even if Husband is correct, it is still ultimately a fact about the lexical meanings of these verbs that inferences of manner and result are introduced simultaneously by the same item.

Finally, it could also be that these verbs do indeed entail both a manner and a result, but they are different somehow from the notions found in pure manner and result verbs respectively, and thus are not subject to complementarity. However, the diagnostics we adopted were rooted in canonical manner and result verbs, and thus it seems clear that the relevant components that give rise to these behaviors are the same. If one wishes to argue that despite this there is some difference in the manner or result entailments so that these do not violate Manner/Result Complementarity, then it is incumbent upon them to provide an explanation of the difference. Absent this, we take the shared behavior of the verbs we have discussed in this section with both canonical manner verbs and canonical result verbs as indicative of shared semantics. Thus these verbs counterexemplify Manner/Result Complementarity as a truth conditional fact of verb meaning (see also Goldberg 2010: 46-50; Rissman 2016; Beavers and Koontz-Garboden 2017a for additional discussion of violations of Manner/Result Complementarity, plus further result verb diagnostics). We next explore how this fact fits into a theory of event structure, ultimately arriving at the conclusion that both meaning components are in the root of each verb. From this it furthermore follows that the semantic relation linking the manner and result together-causation-is also in the meaning of the root, constituting another violation of Bifurcation.

\subsection{Manner, result, and the architecture of event structure}

In the preceding sections we discussed diagnostics for manner and result in a verb's meaning rooted primarily in their truth conditional content, and argued that there are verbs that have both meaning components, counterexemplifying Manner/ Result Complementarity as a truth conditional fact about verb meanings. We now consider the theoretical consequences of this, arguing that complementarity may nonetheless hold as a condition on possible event structures, albeit in ways that allow truth conditional manner+result verbs.

\footnotetext{
${ }^{24}$ That said, Husband's sketch of an analysis is to assume that manner adjuncts and result state complements are integrated into the overall truth conditions defined by the event structure in slightly different ways that derive the assertion vs. presuppositional contrast. However, this requires assuming that the relevant meaning components are introduced by two separate roots, which we show below cannot be the case.
} 


\subsubsection{Complementarity in event structures}

Rappaport Hovav and Levin's explanation for Manner/Result Complementarity relies on properties of event structures. We begin by reconsidering Rappaport Hovav and Levin's Lexicalization Constraint, stated in (56), from which Manner/Result Complementarity is meant to follow as a theorem:

(56) A root can only be associated with one primitive predicate in an event schema, as either an argument or a modifier.

(Rappaport Hovav and Levin’s (p. 25) Lexicalization constraint)

Taking the kind of syntactified event structures we have adopted in previous chapters, this would mean that a root either serves as an adjunct to $v_{\text {act }}$ or $v_{\text {cause }}$ or else serves as the complement of a $v_{\text {become }}$ head, repeating (2a-c):

a.

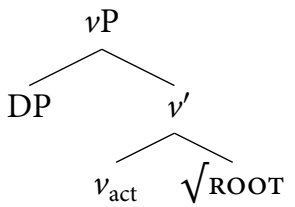

b.
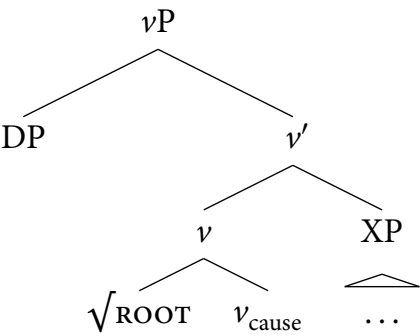

c.

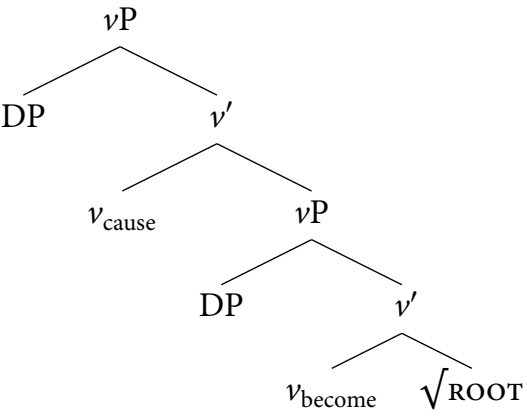


A root cannot somehow do both simultaneously, repeating $(2 \mathrm{~d})$ :

(58)

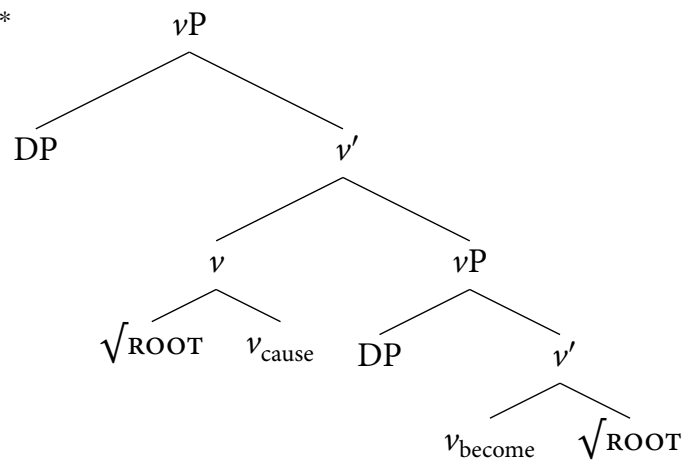

There is a further (implicit) assumption: a surface verb consisting of just one overt morphological root cannot nonetheless have two distinct roots in its event structure, repeating $(2 \mathrm{e})$ :

(59)
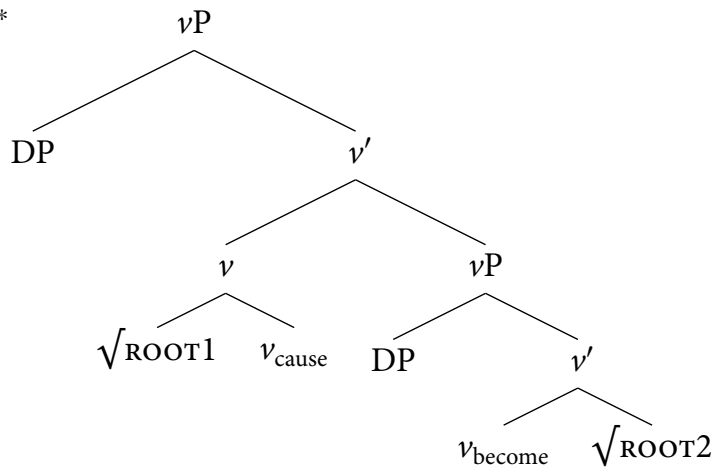

(in a single surface verb)

This event structure is not ruled out a priori, as (per Rappaport Hovav and Levin 1998: 119) it is found with complex resultative constructions as in sweep clean (and perhaps also other complex predicates that Snyder 2001 and Beck and Snyder 2001 assimilate to the same interpretive principle as resultatives). ${ }^{25}$ But Rappaport Hovav and Levin seem to assume that such an event structure cannot

${ }^{25}$ Technically $\sqrt{\text { воот2 }}$ in this case would probably be first Merged with Asp and presumably pos as per $\$ 1.6 .1 .3$, since it surfaces as an adjective. However, the exact functional structure surrounding it does not matter for any claims we make below so we set this issue aside. 
be associated with a single surface verb that has only one overt idiosyncratic morphological root. Otherwise it should be possible to have in essence a "lexical resultative" - a single surface verb that would pattern exactly like a resultative in every way other than having two overt idiosyncratic roots-and this would, by definition, be manner+result entailing.

Thus, Manner/Result Complementarity as a truth conditional claim follows on Rappaport Hovav and Levin's theory from two theoretical assumptions about event structures:

i. There is only ever one root in the event structure of a surface verb showing a single overt idiosyncratic morphological root.

ii. A root's meaning can either contribute a manner or a result state, but not both.

We have shown, however, that complementarity does not hold truth conditionally. Thus if (i) and (ii) do indeed derive it, at least one of them must be wrong. We argue that (i) is correct and (ii) incorrect. To demonstrate this, it is important to realize that (i) and (ii) are not assumptions of the same order. Crucially, (ii) is a claim about how much and what types of truth conditional content can be entailed by a single root (and by extension, a single surface verb consisting of just one morphological root plus functional morphemes). As such, it can be falsified by looking at truth conditional diagnostics, as we have done above (provided we know that we are dealing with a single root in any given predicate, i.e. that (i) holds). Assumption (i), however, is not a truth conditional claim about verb meaning, but rather a claim about how event structures are (de)composed, namely that all of the idiosyncratic meaning in a surface verb showing one overt morphological root is packaged together into one single root element in the event structures. As such the diagnostics needed to validate it may differ from those needed for (ii).

The simplest diagnostic here is one introduced in $\$ 1.3 .2$ (see also $\$ 1.6 .1 .3$ ) and used in both Chapters 2 and 3 to pinpoint aspects of the linguistically significant hierarchical structure of event templates, namely sublexical modification as with modifiers like again. Recall in particular that if again scopes over just the root in a structure like $(57 \mathrm{c})$ for a canonical caused change-of-state verb with a PC root, then the reading is restitutive (the patient held the state denoted by the root before and now holds it again) and if it attaches higher, the reading is repetitive (the patient came to be in that state before and now it comes to be in it again). The analysis of low scoping again over the simple state root $\sqrt{\text { OpEN from }(43) \text { in } \$ 2.4 .1}$ is repeated in (60):

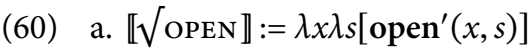

b. $\llbracket$ again $\rrbracket=\lambda P \lambda z \lambda e^{\prime \prime \prime}\left[P\left(z, e^{\prime \prime \prime}\right) \wedge \partial \exists e^{\prime \prime}\left[e^{\prime \prime} \ll e^{\prime \prime \prime} \wedge P\left(z, e^{\prime \prime}\right)\right]\right]$

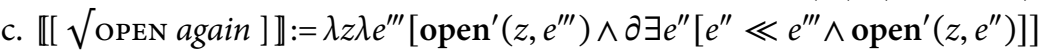


Likewise, recall the semantic opacity of roots assumed by all theories of event structure, whereby roots are scopal units with regard to again no matter how complex the meaning of the root itself (see $\$ 1.4 .3$ ). Thus assuming the template for manner+result verbs is caused change of state, to evaluate whether it is assumption (i) or assumption (ii) above that must be eliminated, we look at these verbs' behavior with scopal modifiers. If manner and result are packaged together in a single root (i.e. assumption (ii) is invalid), scopal modifiers should never scope over one to the exclusion of the other (e.g. restitutive readings should be impossible). If they are entailed by separate roots (i.e. assumption (i) is invalid), then in principle this should be possible.

\subsubsection{The decomposition of transitive manner+result predicates}

We consider first the behavior of manner+result predicates that clearly have two overt morphological roots such as resultative constructions. As Beck and Snyder (2001: 56-7) and Beck and Johnson (2004: 108-10) discuss in detail, in such cases, again can semantically scope over just the result to the exclusion of the manner, as illustrated in (61), something that would follow if resultatives somehow were derived from the event structure in (59).

(61) a. Mary has made a sheet of metal that is flat, but it later accidentally became bent. Fortunately, John hammered the metal flat again.

b. Mary bought a new front door for her house, and installed it in an open position. Later, the wind blew it closed, so John kicked it open again.

c. Mary, a natural red head since birth, decided to dye her hair bright green. However, after seeing herself in the mirror she was mortified, so she went to her hairdresser and he dyed it red again.

This is as predicted if the manner and result are determined by separate roots, with

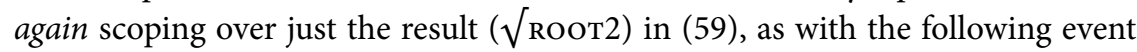
structure for the last clause in (61a):

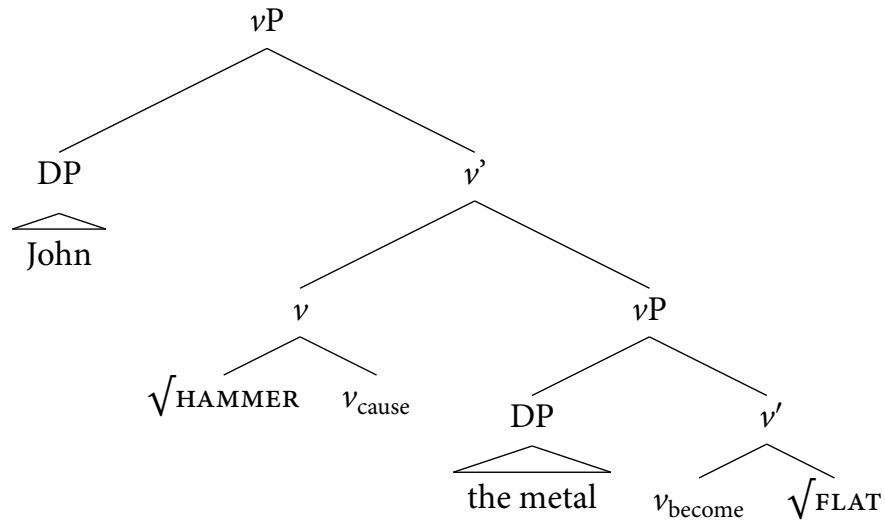


The question is whether this analysis extends to the surface manner+result verbs we identified in $\$ 4.4$, where for example we might treat drown as a lexical resultative with a structure like the one in (63), albeit violating assumption (i), though consistent with (ii):

(63) John drowned Bill.

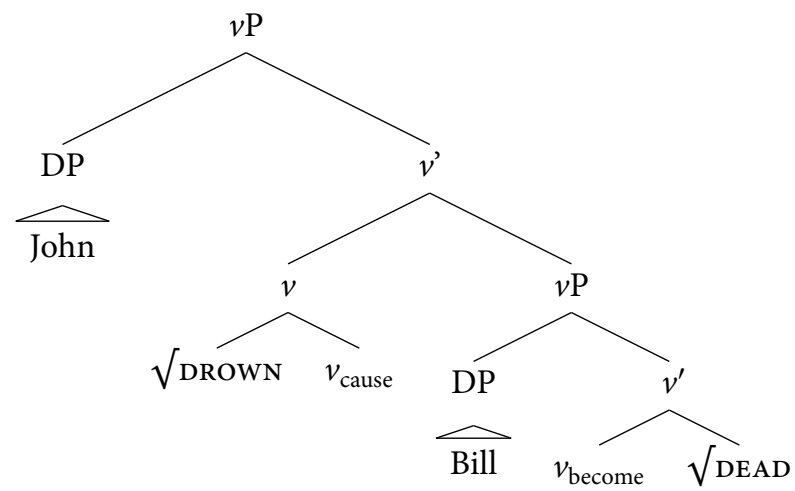

If so, again should be able to scope solely over the result, as with resultatives.

However, again necessarily scopes over both meaning components. To demonstrate this for a verb entailing death, we must appeal again to the somewhat unusual context in $\$ 4.1$ of zombies, which can be killed more than once. Consider an example where a zombie has died and subsequently been reanimated, and John drowns him. Crucially, (64) can only mean that the zombie was drowned some previous time that he died. It cannot mean that he had been killed some other way and is now dying again, this time by drowning:

(64) John drowned the zombie again.

Thus again scopes over both the manner and the result. It cannot scope over just the result as with true resultatives as in (61). Thus putting (64) in a context where this does not obtain yields a contradictory discourse:

(65) [ The only previous time the zombie died was when someone killed it with a chainsaw. It has come to life again, and John kills it by drowning. ]

\#John drowned the zombie again.

Other manner of killing verbs are the same, where in each case in (66) the interpretation is that there was a prior event of actions of the appropriate sort leading to death (for speakers for whom this is the relevant result state): 
(66) a. [ Someone previously hanged the zombie outlaw. It has come to life again, and the sheriff kills it again by hanging. ]

The sheriff hanged the zombie outlaw again.

b. [ Someone previously crucified the zombie leader. It has come to life again, and the Romans kill it again by crucifixion. ]

The Romans crucified the zombie leader again.

c. [ Someone previously electrocuted the zombie prisoner. It has come to life again, and the executioner kills it again by electrocution. ]

The executioner electrocuted the zombie prisoner again.

d. [ Someone previously guillotined the monster. It has come to life again, and the executioner kills it again by using a guillotine. ]

The executioner guillotined the monster again.

Embedding these in contexts where death did not obtain by the stated manner leads to unacceptability:

(67) a. [ The only previous time the zombie died was when someone killed it with a chainsaw. It has come to life again, and the sheriff kills it again by hanging. ]

\#The sheriff hanged the zombie outlaw again.

b. [ The only previous time the zombie died was when someone killed it with a chainsaw. It has come to life again, and the Romans kill it again by crucifixion. ]

\#The Romans crucified the zombie leader again.

c. [ The only previous time the zombie died was when someone killed it with a chainsaw. It has come to life again, and the executioner kills it again by electrocution. ]

\#The executioner electrocuted the zombie prisoner again.

d. [ The only previous time the monster died was when someone killed it with a chainsaw. It has come to life again, and the executioner kills it again by using a guillotine. ]

\#The executioner guillotined the monster again.

If manner of killing verbs are lexical resultatives, this is all entirely unexpected, since it should be possible to simply get death (or whatever state it is) under the scope of again and nothing else.

Cooking verbs are identical, though as in $\$ 2.4 .1$ it is difficult to establish plausible contexts in which the relevant result state can obtain twice for the same food item. However, the degree to which poach, sauté, and braise admit degree achievement readings-wherein the relevant result state must only be obtained to 
some degree but not necessarily a maximal degree-then it might be possible to get a supposedly restitutive reading where some degree of the result obtained before and now some (new) degree of that result obtains again, e.g. as with cool (on an atelic reading as per $\$ 1.6 .1 .2)$ :

(68) [ John had previously cooled the soup down to 100 degrees by putting ice in it, after which he used a burner to maintain that temperature. He then put ice in it again to cool it down some more. ]

John cooled the soup again.

Note that the manner of putting ice in the soup in (68) need not be repeated again. John could have cooled the soup the first time by blowing it with cold air and the example sentence in (68) would be just as acceptable.

With manner of cooking verbs we see a different pattern. In a context where the food item in question had previously been at least partly put into the relevant state of cookedness, the reading for all of (69) is that the result obtained more by virtue of the same cooking process that had occurred previously, not just that the food came to be more in the same cookedness state again regardless of the process:

(69) a. [ Someone previously submerged the pears in a small amount of simmering liquid, which made them soft, and then Jane does this again. ] Jane poached the pears again.

b. [ Someone previously placed the meat in a pan of hot oil over fire to let it brown some, and then Jane does this again. ] Jane sautéed the meat again.

c. [ Someone previously seared the tofu and then placed it in a covered pan in a hot oven in flavored liquid, which gave it a softer texture. ] Jane braised the tofu again.

In contexts where the processes differ, these sentences are infelicitous:

(70) a. [ Jane had previously made the pears adopt a softer, cooked texture by putting them in a steamer over boiling water. They were not perfectly soft, so she then placed them in a small amount of simmering liquid, which made them even softer. ] \# Jane poached the pears again.

b. [ Jane had previously made the meat have crisp, fatty, brown edges by applying a blowtorch. The meat was not fully browned or cooked through, so she then placed it in a pan of hot oil over fire let it brown some more. ]

\#Jane sautéed the meat again. 
c. [ Jane had previously seared the tofu and then placed it in a steamer over a boiling, flavored liquid, giving it a softer texture. The tofu had an unfinished, uneven texture and was not cooked on the inside, so she seared it again and placed it in a covered pan in a hot oven in flavored liquid, which evened out the texture and cooked it more thoroughly. ] \#Jane braised the tofu again.

In all cases, it is not possible to separate out the result state-whatever that happens to be for a given verb-from the process that leads to the result state. This behavior is unlike proper resultatives, where the result state is separable from the stated process, suggesting that these verb are not lexical resultatives.

However, it might be possible to maintain the lexical resultative analysis, but posit that these verbs idiosyncratically disallow restitutive attachment of again, leaving only repetitive attachment and thus only a repetitive reading. As we discussed for result root verbs in $\$ 2.5 .3$, this analysis is problematic. First, it is stipulative-why would manner of killing and cooking verbs but not canonical resultatives require this if they have the same event structures, especially given that postsentential again otherwise generally allows restitutive attachment? Second, just as with result root verbs in $\$ 2.4 .1$, the facts here repeat themselves with scopal $r e$ - modification which, as we noted earlier, is always low scoping. Thus if manner of killing and cooking verbs are lexical resultatives, $r e$ - should either show a purely restitutive reading or be ruled out altogether since these verbs seem to force high attachment of scopal modifiers. However, attaching re-to guillotine in the zombietype context produces exactly the same reading as again in (64), and attaching reto braise gives rise to the same reading as braise again in (69c) (we thank Alec Marantz, p.c., for pointing this and its significance out to us):

(71) a. John reguillotined the zombie.

b. John rebraised the tofu.

This rules out contexts where the manner and result did not both obtain:

(72) a. [ The only previous time the monster died was when someone killed it with a chainsaw. It has come to life again, and the executioner kills it again by using a guillotine. ]

\#John reguillotined the zombie.

b. [ Jane had previously seared the tofu and then placed it in a steamer over a boiling, flavored liquid, giving it a softer texture. The tofu had an unfinished, uneven texture and was not cooked on the inside, so she seared it again and placed it in a covered pan in a hot oven in flavored liquid, which evened out the texture and cooked it more thoroughly. ] \#John rebraised the tofu. 
This is surprising if $r e$ - only has restitutive attachment. One could say that $r e-$ does allow repetitive attachment, and that manner+result verbs categorically require this attachment for the same reason they require it of again. However, this still requires some additional mechanism to rule out a restitutive reading, which is otherwise attested with resultatives. We turn next to an alternative analysis that instead maintains assumption (i) but rejects (ii), and show that it captures all of the above data directly without any additional mechanisms.

\subsubsection{Roots of transitive manner+result verbs as complements of $v$}

An alternative would be that the verbs we have identified as transitive manner+result verbs allow restitutive attachment (thus accommodating the standard assumptions about $r e-$ ), but restitutive attachment gives rise to a repetitive reading. How could this be? We had in fact discussed exactly this sort of situation in $\$ 2.4 .1$ with regard to result root verbs, e.g. verbs like crack whose roots entail change of state as well as the final state the patient ends up in. There we suggested that a root

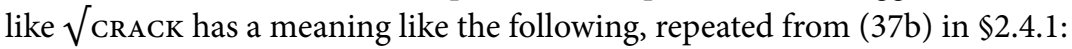

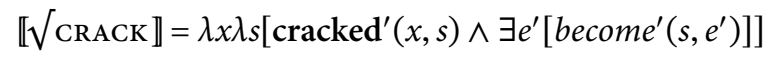

When such a root is placed under the scope of again at a restitutive attachment site the reading will be not just that the patient came to be in the relevant state but that the state had obtained before by virtue of some change of state.

It seems that manner+result roots are of exactly the same class, but with an additional requirement above and beyond that there be a change of state, namely that there is also a specific process that gives rise to that change of state. One might object that this requires a root that is complement to $v_{\text {become }}$ to impose constraints on the verb's subject, the specifier of $v_{\text {cause }}$, despite Kratzer's (1996) claim that heads below the one that introduces external arguments cannot impose conditions on it. However, nothing a priori rules out a root not just describing a result state but also that (a) the result has a cause and (b) the cause is of a certain type (see Wechsler 2005b for a general critique of Kratzer's proposal along these lines). This is in fact just the mirror image of what we proposed for ditransitive roots in $\$ 3.5$, where a manner attaching root was able to impose conditions on arguments of the lower $\mathrm{P}$ head by placing constraints on whatever happened to be the theme and recipient of the result state complement of the $v_{\diamond \text { cause }}$ the root modifies (for more see $\$ 4.5 .4)$.

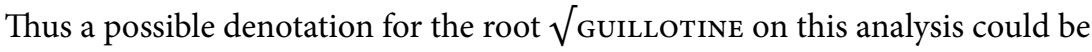
(74), where it predicates a result dead' of its single individual argument, but also says that it must have a cause, and further that any such cause is of a certain type, namely a guillotining-type action: 


$$
\begin{aligned}
& \llbracket \sqrt{\text { Guillotine } \rrbracket}=\lambda x \lambda s\left[\operatorname { d e a d } ^ { \prime } ( x , s ) \wedge \exists e ^ { \prime } \exists v \left[\operatorname{cause}^{\prime}\left(v, e^{\prime}\right) \wedge\right.\right. \\
& \left.\left.\operatorname{become}^{\prime}\left(s, e^{\prime}\right) \wedge \forall v^{\prime}\left[\operatorname{cause}^{\prime}\left(v^{\prime}, e^{\prime}\right) \rightarrow \operatorname{guillotining}^{\prime}\left(v^{\prime}\right)\right]\right]\right]
\end{aligned}
$$

Although this root does not predicate directly of the actual causing event introduced by $v_{\text {cause }}$, it does impose a constraint that any event that caused $s$ must have been a guillotining-type action, thus ensuring that whatever specific event caused $s$ must have been a guillotining-type action, in essence indirectly constraining the actual manner of causation. Note that it is not sufficient for the root to say of event $v$ that it be a guillotining-type action, since there is no assurance that the actual causing event introduced by the predicate denoting causation will be equated with $v$.

The analysis in (74) in turn derives that these verbs will pass manner diagnostics, since this condition ensures (a) that the subject must meet certain conditions as per $\$ 4.3 .1$, taking the assumption from $\$ 1.6 .2 .1$ that constraining an event to be a certain type of action guarantees that its sole effector participant is the one performing that action, (b) more generally that there is a non-cancelable inference that some action occurred as per $\$ 4.3 .2$, and (c) that if the action is sufficiently complex durativity will result as per $\$ 4.3 .3$. It also ensures that the verb is still nonetheless a result verb since there is a non-cancelable scalar result state as per $\$ 1.6 .1$ and thus should pattern as in $\$ 4.2$, assuming appropriate semantics-tosyntax conditions on result verbs to ensure proper object deletion and resultative licensing properties.

Crucially, if (74) were the meaning of the guillotine root, applying again as defined in (60b) generates the meaning that there is a state of death that is necessarily caused and the causing event is a guillotining-type action, by virtue of the fact that anything that caused this state is a guillotining-type action, and furthermore there is a presupposition that there had been a prior state satisfying all the same conditions, so that it was also a state of death, it had a cause, and that cause was a guillotining-type action:

$$
\begin{aligned}
& \llbracket\left[\sqrt{\text { GuIllotine again }] \rrbracket}=\lambda z \lambda e^{\prime \prime \prime}\left[\operatorname { d e a d } ^ { \prime } ( z , e ^ { \prime \prime \prime } ) \wedge \exists e ^ { \prime } \exists v \left[\operatorname{cause}^{\prime}\left(v, e^{\prime}\right) \wedge\right.\right.\right. \\
& \left.\operatorname{become}^{\prime}\left(s, e^{\prime}\right) \wedge \forall v^{\prime}\left[\operatorname{cause}^{\prime}\left(v^{\prime}, e^{\prime}\right) \rightarrow \operatorname{guillotining}^{\prime}\left(v^{\prime}\right)\right]\right] \wedge \partial \exists e^{\prime \prime}\left[e^{\prime \prime} \ll e^{\prime \prime \prime} \wedge\right. \\
& \operatorname{dead}^{\prime}\left(z, e^{\prime \prime}\right) \wedge \exists e^{\prime} \exists v\left[\operatorname{cause}^{\prime}\left(v, e^{\prime}\right) \wedge \operatorname{become}^{\prime}\left(e^{\prime \prime}, e^{\prime}\right) \wedge\right. \\
& \left.\left.\left.\forall v^{\prime}\left[\operatorname{cause}^{\prime}\left(v^{\prime}, e^{\prime}\right) \rightarrow \text { guillotining }{ }^{\prime}\left(v^{\prime}\right)\right]\right]\right]\right]
\end{aligned}
$$

Thus while (74) does not directly predicate of the causing event, its meaning restricts the possible interpretations of that event.

An analysis of this sort is not unheard of. Marantz (2009) makes an analogous argument for an apparently repetitive re-reading with read:

(i) The man reread the book. 
The default reading is that the same man read the book before, as though rescopes over the subject. However, Marantz suggests that this arises because the read root entails a separate, unexpressed reader participant that is (almost always) interpreted coreferentially with the subject. This gives the appearance that the man is in the scope of $r e-$, when it technically is not. This is similar to our analysis for manner of killing and cooking roots, where the relevant entity is instead the causing event.

Our analysis thus allows us to maintain that again and re-show restitutive attachment with manner of killing verbs, as expected if they have caused change-of-state event structures. Thus unlike the analysis in $\$ 4.5 .2$ that maintained assumption (ii) but eliminated (i), no additional mechanisms are needed to rule any readings out. Restitutive readings do not arise because restitutive attachment generates a repetitive reading due to the idiosyncratic meaning of the root, exactly as we had proposed for result roots in \$2.4.1. Our analysis also predicts that again cannot scope over the manner in a manner of killing verb to the exclusion of the result. This again follows from the fact that the manner is packaged with the result in the root, which acts as a scopal unit. In sum, the roots of manner of killing and cooking verbs are a subtype of the broader class of result roots from Chapter 2, where they entail not just that the state must have come about but also that there be a process that caused it, all from the position of a root that describes a result state as is commonly the root of transitive change-of-state verbs.

That said, a reviewer asks whether the same results could be obtained by assuming that the guillotining in (74) is predicated over become events rather than causing events (i.e. that for any event $e^{\prime}$ that stands in a become $e^{\prime}$ relation with $s$ the event $e^{\prime}$ must be a guillotining). As per $\$ 1.6 .2 .1$ we take the general assumption that since the manners we are discussing here pertain to actions the effector takes in bringing about the result then they apply to the causing event since the subject is a participant in that event (but that if the manner entailments were about the patient instead, then they might be more appropriately applied to the become event; see fn. 10). However, this does not preclude the possibility that it is in fact the become event that is constrained in (74), provided some conditions ensure that it is still the subject upon whom appropriate mannerrelated selectional restrictions apply. Otherwise, the other manner diagnostics in $\$ 4.3$ do not depend on how exactly the manner is part of the larger truth conditional content of the verb vis-a-vis its event structure. Either way, the same results obtain, though again the subject selectional restrictions do suggest that the causing event is the appropriate place for these conditions to be imposed (see also $\$ 4.6$ for additional discussion of this point). We next ask whether the same scope facts obtain for the other class we discussed in $\$ 4.4$, namely ditransitive ballistic motion verbs. 


\subsubsection{Roots of ditransitive manner+result verbs as adjuncts of $v$}

Recall from Chapter 3 that for ditransitive verbs, including ballistic motion ditransitives, the root sits in a manner position not in a result position:

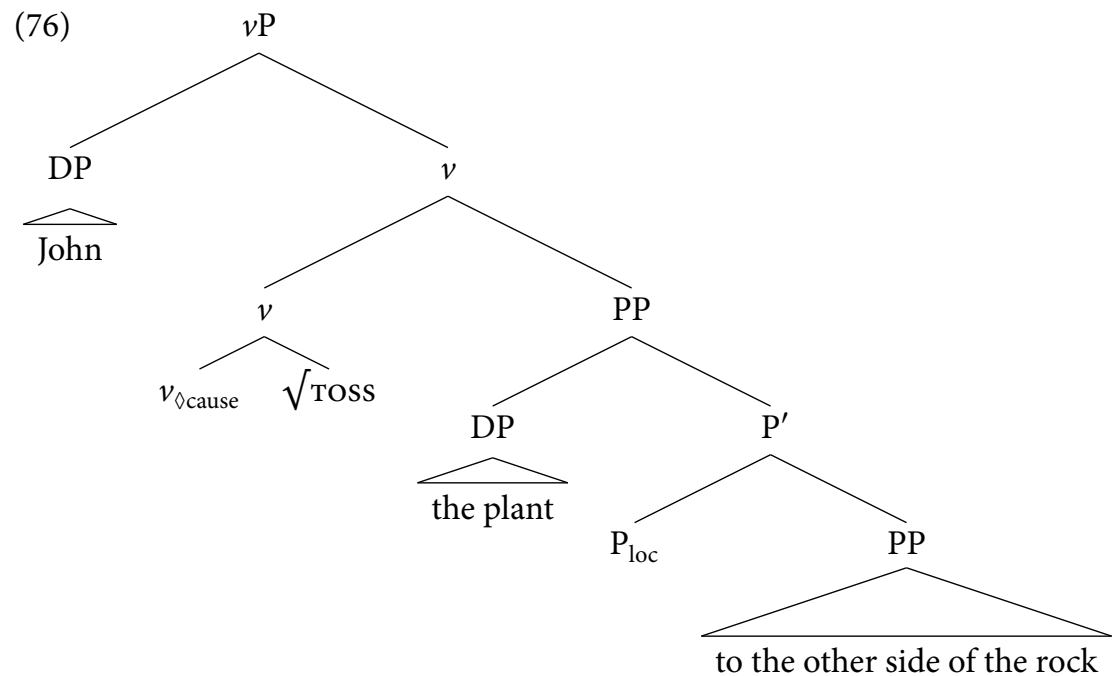

Part of the evidence for this comes from restitutive modification facts, as discussed in \$3.9. In particular, Beck and Johnson (2004: 108-10) showed that both variants of the dative alternation allow restitutive again as with toss and carry in the to frame in (77) from $\$ 3.9:^{26}$

(77) [ A plant grew on the opposite side of a rock from where John is standing. He picks it, then tosses it back to its original location. ] John tossed the plant [ to the other side of the rock again ].

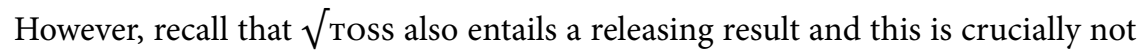
under the scope of again, which suggests that whatever generates the releasing is higher in the structure than the result-denoting $\mathrm{P}$ head, exactly as predicted on the analysis assumed here. But the question before us now is where manner is

${ }^{26}$ In $\$ 3.9$ we also discussed restitutive possession in indirect object frames, albeit using give. However, to construct the appropriate context for restitutive readings-which would require a state that had held before but was never caused to come about-it is best to use inalienable possession as the initial state. However, our focus here is on ballistic motion verbs and it is difficult for us to think of a context of inalienable possession being restored through an act of ballistic motion. Thus we focus here just on to frames and co-location. 
introduced, e.g. where whatever it is that defines toss as distinct from hurl comes from.

Crucially, just as the releasing is not under the scope in (77), neither is the tossing manner that goes with it, i.e. there is no prior tossing action in (77) and indeed no prior action at all. This predicts furthermore that had there been a prior action with a distinct manner the example sentence in (77) should be fine, and it is:

(78) [ Mary forcibly hurls a plant to the other side of the rock. John picks it up and then gently tosses it back there. ]

John tossed the plant [ to the other side of the rock again ].

Furthermore, for the other two syntactic positions of again-presentential and preverbal—a repetitive reading necessarily obtains (see $\$ 2.4 .1,(49)$ ), and in these cases both the tossing-type action and the releasing must have occurred before. Any context in which it is not the case that both of these conditions obtain does not yield a felicitous sentence. Thus in (79a) there was no prior event at all (i.e. the context would only support a restitutive reading) and presentential and preverbal again are out, while in (79b) there was prior action by John but it did not involve release or tossing and in (79c) there was prior action by John but while it involved releasing it did not involve tossing, and in both cases the presentential and preverbal again are out. ${ }^{27}$ The only context supporting again in those positions is $(79 \mathrm{~d})$ where there was a prior tossing and releasing.

(79) a. [ A plant grew on the opposite side of a rock from where John is standing. He picks it, then tosses it back to its original location. ] \#(Again) John (again) tossed the plant to the other side of the rock.

b. [ John is holding a plant and places it on the other side of a rock, not taking his hand off it. He then lifts it back up in the air, and then tosses it gently so that it lands on the other side of the rock. ]

\#(Again) John (again) tossed the plant to the other side of the rock.

c. [ John is holding a plant, and then forcibly hurls it to the other side of a rock. He then picks it up and gently tosses it to the other side of the rock. ]

\#(Again) John (again) tossed the plant to the other side of the rock.

d. [ John is holding a plant, and then gently tosses it to the other side of a rock. He then picks it up and for a second time gently tosses it to the other side of the rock. ]

(Again) John (again) tossed the plant to the other side of the rock.

\footnotetext{
${ }^{27}$ We find it impossible to construct a context of a tossing manner with no releasing (see $\$ 5.2 .1$ ), so we do not test for this condition.
} 
Thus on a restitutive reading the releasing plus tossing are outside of the scope of again but on all repetitive readings they are both underneath it. On no reading is the tossing manner separable from the releasing result. These are exactly the facts that would arise if the root contains both meaning components, so that scoping over one means scoping other the other, but it was in manner adjunction position and thus outside the scope of restitutive modification (see also $\$ 3.10$ for more on restitutive modification with ditransitives in general). ${ }^{28}$

\subsubsection{A typology of the roots of manner+result verbs}

In sum, the lack of restitutive readings for manner+result verbs is naturally accommodated if we assume that these verbs do indeed only contribute one root at a time, maintaining assumption (i) in $\$ 4.5 .1$, but this root entails both a manner and a result simultaneously, dispensing with assumption (ii). This yields at least three different types of root with regards to result verb and manner verb entailmentsroots that describe states (e.g. for redden, destroy), roots that describe manners (e.g. for run, jog), and roots that do both simultaneously (e.g. manner of killing verbs, cooking verbs, ditransitive ballistic motion verbs). This new type of root in turn guarantees a new type of verb class, namely one that entails manner and result at the same time, regardless of what kind of template it occurs in. We return to the larger question of root typologies in more detail in $\$ 5.4$. The alternative to our analysis-allowing multiple roots in a lexical resultative structure-requires additional mechanisms to rule out restitutive readings otherwise attested with resultative event structures. Thus, while each approach involves dropping one of Rappaport Hovav and Levin's assumptions, the approach wherein roots can entail both a manner and a result state at the same time is preferable, as it requires no additional assumptions.

Indeed, given that manner and result are two independently attested types of meanings truth-conditionally, the null hypothesis should be that there are roots entailing both (consistent with Grimshaw's 2005: 85 claim that verbs are not constrained in terms of the complexity of the truth conditions they entail). Furthermore, this approach brings manner of killing verbs, cooking verbs, and ditransitive ballistic motion verbs in line with the attested result roots in Chapter 2, making predictions about which types of adjectival forms should exist for such roots. In particular, the prediction is that the verbs we have discussed in this chapter should lack simple adjectival forms, and this is universally the case. Thus while

\footnotetext{
${ }^{28}$ That said, it would technically be possible to maintain an analysis wherein somehow the manner and the releasing were introduced by separate functional projections but that somehow again, owing to its various possible attachment sites, can never target just one of those meanings without also scoping over the other. However, there would be no empirical support for this analysis and thus little reason to assume it.
} 
there are deverbal adjectives of all of the manner+result verbs (e.g. guillotined, drowned, braised, poached, tossed, thrown) there are no simple adjectives of any sort. We turn next to an additional fact about these roots that suggests that not only do they violate the tenets of Manner/Result Complementarity, they also violate Bifurcation.

\subsection{The roots of manner+result verbs and Bifurcation}

So far in this chapter we have mostly been concerned with Manner/Result Complementarity, i.e. that a single surface verb built from one overt idiosyncratic morphological root should not entail both manner and result at the same time. We have shown that this is false, though we have also shown that verbs that violate it have just one root in their event structures. This one root is what is responsible for introducing the entailments that give rise to a verb being a manner+result verb. But a deeper question is why both an action and a result state would be described by a single root. Functionally, as we noted above, given single rootedness in event structures a root entailing both would be the only way to ever have a single surface verb entailing both while still maintaining that there is just one root per verb. However, there is also a quite simple formal explanation for this: what these roots ultimately entail is causation, and the manner and the result simply represent entailments the root has regarding the causing and caused events respectively that are an inherent part of the meaning of causation and thus come for free with it.

In particular, not only are the manner and result both universally found under the scope of again in transitive manner+result verbs, so is the causal relation connecting them, something that is in fact predicted by the formal analyses we sketched above, since the roots in all cases contained entailments about causation in them. Consider again the verb drown with postsentential again. It is not sufficient that manners or actions of the sort associated with someone drowning as well as that person's death are what occurred before. It must also be the the case that those previous actions lead to the previous death of the patient. Thus (80) is not possible in a context where the relevant action and result obtained previously but there was no causal link between them (setting aside exactly how one defines a causal link, e.g. through some dependency or production theory; see $\$ 1.6 .2 .1$ ):

(80) [ A zombie is held under water and water enters his lungs, but he survives and is later killed by a bullet to the head. After being reanimated, John holds him under water until his lungs fill with water and he dies again. ]

\#John drowned the zombie again.

The same goes for other manner of killing verbs above (at least for speakers for whom these verbs entail death and not just a state that could lead to death): 
(81) a. [ A zombie has a noose put around his neck and is dropped from a tree. However, he is removed from the noose before he dies and is instead later killed by a bullet to the head. After being reanimated, the sheriff puts a noose around his neck and throws him off a tree, this time snapping his neck and killing him. ]

\#The sheriff hanged the zombie outlaw again.

b. [ A zombie has been nailed to a cross that is subsequently hoisted up. However, he is taken down and released before he dies and is instead later killed by a bullet to the head. After being reanimated, the Romans nail him to a cross and hoist it up, leaving him there until he dies. ]

\#The Romans crucified the zombie leader again.

c. [ A zombie has been administrated an extreme electrical shock. However, the shock is stopped before he dies, though he is later killed by a bullet to the head. After being reanimated, the executioner applies another extreme electrical shock, this time killing him. ]

\#The executioner electrocuted the zombie prisoner again.

d. [ A monster has been placed in a guillotine and the blade was dropped but it misses and he escapes alive. He is later killed by a bullet to the head. After being reanimated, Dr. Frankenstein puts him back in the guillotine and drops the blade, this time severing his head and thus killing him. ]

\#Dr. Frankenstein guillotined the monster again.

Cooking verbs are the same: applying the method described by the verb and independently achieving the result described by the verb but with no causal link between the two does not license the possibility of again modification:

(82) a. [ Jane placed the pears in simmering liquid, but quickly took them out before anything changed about them. She then made the pears adopt a softer, cooked texture by putting them in a steamer over boiling water. They were not perfectly soft, so she then placed them in a small amount of simmering liquid, which made them even softer. ]

\#Jane poached the pears again.

b. [ Jane placed the meat in a pan of hot oil over fire but quickly removed them before anything changed about them. She then made the meat have crisp, fatty, brown edges by applying a blowtorch. The meat was not fully browned or cooked through, so she then placed it in a pan of hot oil over fire let it brown some more. ]

\#Jane sautéed the meat again.

c. [ Jane put the tofu in a hot pan and then stuck it in the oven in flavored liquid, but in both cases quickly removed them before anything changed 
about them. She then took the tofu, seared it, and placed it in a steamer over a boiling, flavored liquid, giving it a softer texture. The tofu had an unfinished, uneven texture and was not cooked on the inside, so she seared it and placed it in covered pan in a hot oven in flavored liquid, which evened out the texture and cooked it more thoroughly. ] \# Jane braised the tofu again.

In the case of ballistic motion verbs, of course, the claim is that the root is outside the scope of restitutive modification, and thus neither the manner nor the result is under the scope of again, as shown in (77), repeated here:

(83) [ A plant grew on the opposite side of a rock from where John is standing. He picks it, then tosses it back to its original location. ] John tossed the plant [ to the other side of the rock again ].

Crucially, there is also no entailment of any sort of prior causation (an unsurprising fact given that there was also no prior event of the appropriate sort to count as causing and caused events anyway, i.e. tossing-type actions and releasing). This again suggests that the entailment of causation that links the action and the coming about of the result state is in the meaning of the root.

A reviewer does raise an objection here: consistent with $\$ 4.5 .3$ what if the relevant manner entailments were placed on the become event and not the cause event in the root? In this case what rules out all of these examples is that a become event with the appropriate manner and result did not obtain before, and there is no need to appeal to causation in the root meaning at all. However, as noted in $\$ 4.5 .3$, while this is technically possible it would involve somehow ensuring that conditions placed on the become event result in selectional restrictions on the subject as per $₫ 4.3 .1$. This could be achieved in one of two ways: the relevant manner conditions could truth conditionally ensure that for any event that causes the given change its effector must act in a certain way, or the subject could somehow be a participant in the become event as well as the causing event and then the conditions on the become event would apply to that participant. In the former case, though, the truth conditions for the manner are still defined in terms of causation since a causing event must be identified, and thus causation is again in the meaning of the root. In the latter case it is not clear what role the subject would have in the become event other than being an effector qua causer, which would again mean that causation will be part of ultimately identifying the participant who performs the manner that comes from the root. Thus in either case, fundamentally the manner must involve a root-introduced notion of causation.

In sum, these results mean that in addition to change, possession, co-location, and the other templatic entailments we have argued in the previous chapters to be 
possible lexical entailments of roots causation is also a possible root entailment. Given that we have largely exhausted the class of generally accepted templatic meanings, this suggests that there is little support for even a more limited form of Bifurcation.

\subsection{Roots vs. templates in manner+result verbs}

Before concluding, we return once again to the issue raised in $\$ 1.4 .3, \S 2.6$, and $\$ 3.10$ of a justification for a root vs. template distinction for the verbs discussed here in light of the fact that the meanings of the roots that defy Manner/Result Complementarity wholly subsume the meanings of the templates they occur in. As in previous chapters, we suggest that templates still have a role in introducing certain types of semantically conditioned morphosyntactic structure that roots do not even when the root entails the same meaning as the template that determines such structure.

First, recall from $\$ 3.10$ that among ditransitives, including the ballistic motion verbs we have discussed here, it is the template and not the root that determines the asymmetric c-command relationships between the first and second complements within such constructions. Even roots whose meanings wholly subsume the meaning of a given template do not guarantee the c-command relationships between the two complements that that template does. We can see the same, in fact, among the roots of transitive change-of-state verbs we have discussed in this chapter. In particular, the causative template in $(57 \mathrm{c})$ predicts the fact discussed in $\$ 1.3 .1$ (modulo objections in fn. 4 in that section) that causers are syntactically more prominent than patients with caused change-of-state verbs (i.e. causers asymmetrically c-command patients). But as we discussed in $\$ 4.6$, some roots entail caused changes of state as well. Yet simply introducing this meaning does not ensure that there must be a causer DP c-commanding a patient DP, or even any causer overtly expressed at all. For example, stative uses of manner+result roots only take a patient argument as in The tofu is braised ( ${ }^{\star}$ by John). Thus while the root introduces the same meaning as $v_{\text {cause }}$, it is only when an actual $v_{\text {cause }}$ is present that we see the prominence of causers over patients. The conclusion is therefore clear that templates do more than just introduce templatic meaning. While roots and templates can introduce the same kinds of meanings, only templates provide argument structure that figures into asymmetric c-command.

A similar argument can be made for the role of templates in providing semantic structure from the facts above regarding again modification for transitives in $\$ \$ 4.5 .2-4.5 .3$ and $\$ 4.6$. In those cases it was crucial that the lowest scope position for again was over the entirety of what was in the root meaning, including the manner, the result, and the causal link between them. This was unlike (say) true 
resultatives in $\$ 4.5 .2$, where the result could be separated from the manner. This suggests that even though the roots here introduce the meaning of an entire template (the one that generates causative resultatives) only the template provides the appropriate semantic structure to license the scopal attachment points to separate out the various meanings licensed by both. Similarly, for the ditransitives in $\$ 4.5 .4$ no attachment point of again clearly separates out either the root-supplied manner or the root-supplied result seperate from the other. Only when such meanings are introduced by distinct heads within a template as with resultatives can they be probed for separately by sublexical modifiers. Thus the roots we discussed here do not define decomposed semantic constituents accessible to sublexical scopal modification, just as they do not define structural asymmetries between arguments.

\subsection{Concluding remarks}

In the preceding chapters we addressed the question of the Bifurcation Thesis of Roots, the thesis that roots cannot have the same lexical entailments as templatic heads, and suggested that it does not hold for a range of canonical and less canonical templatic entailments. In this chapter we turned to a different type of limit on root meaning, namely how many types of idiosyncratic meaning a root can have. We specifically considered Manner/Result Complementarity, by which (among other conditions on possible verb meaning) a given root cannot have both action and result state entailments in it. Based on diagnostics for result and manner-scalar change/result and non-scalar change respectivelyin the meaning of verbs, we argued that this is not true, and that roots exist with both components, though ultimately those roots are a subset of those that have causation as part of their meaning. The existence of such roots derives new predictions about possible verb types, which we return to in $₫ 5.4$. These data in turn derive a further counterexample to Bifurcation, namely the quite uncontroversially templatic entailment causation, the semantic glue that relates the manner and result aspects of manner+result roots.

One dangling issue though is the single-rootedness condition we argued for above, i.e. the claim that event structures for surface verbs with one overt idiosyncratic morphological root can only have one root element in them (or conversely the roots in event structures cannot have null instantiation). This was key to the argumentation that the roots of manner+result verbs have both action and state entailments in them. But does this assumption itself follow from anything? As far as we can tell it is a stipulation, since there is no a priori reason why a surface verb with one overt root could not be associated with an event structure that idiosyncratically specifies both a manner and a result root. However, whatever 
determines it, the facts with manner+result verbs follow, since it entails that all of the idiosyncratic meaning of a verb is packaged into a single root. Thus while Manner/Result Complementarity is not a truth conditional fact about word meaning per se, it is a structural one following from the number of roots an event structure can have for any one surface verb exhibiting just one overt morphological idiosyncratic root. We leave a deeper explanation for this fact to future work. 



\section{5 \\ Conclusion}

\subsection{Summary on conditions on root meaning}

In this book we have aimed to shed light on the nature of verb meaning and constraints on it. Taking as our point of departure the widely accepted view that the meanings of verbs follow from a structured representation-an event structurewe have examined the interplay between those elements of a verb's event structure responsible for its broad meaning-the template-and those responsible for its idiosyncratic nature-the root-with a goal of understanding the extent to which event structures constrain possible verb meanings. Our focus has been on the semantics of roots, the part of the event structure that has hitherto been less studied, but an understanding of which is crucial to realizing the predictions event structures make on the question of possible verb meanings, as originally discussed by Dowty (1979: 125-9).

We considered two recent proposals about possible root meanings. The Bifurcation Thesis of Roots has it that there is a significant division of labor in terms of what kinds of meanings can be introduced into a given event structure by the template vs. the root. Semantic notions such as cause and change that are in some cases introduced templatically can only be introduced templatically, and are excluded from the meanings of roots. Instead roots introduce only idiosyncratic, non-templatic information, mostly centered around particular states or actions on a verb-by-verb basis, filling in idiosyncratic details of particular templates associated with broader classes of verbs such as activities or change-of-state verbs. The second hypothesis is Manner/Result Complementarity, the claim that among non-templatic, idiosyncratic content there is a prohibition against describing too many distinct types of meaning. While it is widely accepted that roots may describe actions or states that are the results of (templatically-defined) changes of state, no root can describe both at the same time. Adding to this the condition that there can be only one root per event structure for a verbal predicate with just one morphological root, a division of verbs into manner and result verbs follows.

We have argued against both claims. Through a study of change-of-state verbs and ditransitive verbs of caused possession we have shown that many notions canonically assumed to be introduced by templatic heads-causation, change, possession, co-location, possibly among others-can be found in the meanings of particular roots. The evidence for this has come from showing that once we factor out the meaning of a template for a given verb, we still see evidence for these 
templatic notions being introduced somehow that is orthogonal to or in parallel with whatever the meaning of the template is. This meaning must therefore be coming from the root. Thus some change-of-state verbs have roots that introduce cause and change, something that can be further supported through the use of sublexical scopal modifiers that pick out root meanings to the exclusion of templatic meaning. Similarly, ditransitive verbs have templates that are quite weak in the broad eventive meaning they introduce. The choice of the root seems to figure into whether notions such as possession or co-location are entailed by the surface verb, suggesting that these meanings are also coming from the root. This is reinforced again by sublexical modification facts that isolate the templatic meanings from the root meaning, plus a variety of other aspectual and argument realizational properties of ditransitives.

The data all together suggest that Bifurcation does not hold. Roots can introduce templatic meaning just as templates can, and do so in ways that derive a surface verb meaning identical to those that would have the relevant templates. As for Manner/Result Complementarity, similar data have shown that the roots of at least some transitive change-of-state and ditransitive verbs describe both a particular action and a particular (result) state, explaining why some surface verbs entail both meanings despite having event structures with a single root in them.

In this chapter we synthesize the results from the preceding chapters, and return to the question of what constraints, if any, event structures place on possible verb meanings and what grammatical correlates follow from this, especially as are derived from constraints on possible root meanings. We begin in $\$ 5.2$ by considering what factors would give rise to roots that defy Bifurcation and Manner/Result Complementarity, focusing on how the ways some states and actions are conceived of derive some violations of these principles, while in other cases conventional or common associations of certain kinds of concepts result in lexicalization of roots describing both. We consider further possible constraints on root meaning in $\$ 5.3$, tentatively coming to the conclusion that there may well not be any particular constraints on what a root can mean. Despite this somewhat negative conclusion about constraints on root meanings, in $\$ 5.4$ we pull together the root classes we proposed throughout this study and show that they form a typology of possible root meanings that in turn derives a predictive theory of possible verbs in terms of their combined grammatical and semantic properties.

\subsection{The origins of complex root meanings}

Meanings that violate Bifurcation and Manner/Result Complementarity were largely stipulated in the representations given in preceding chapters. For example,

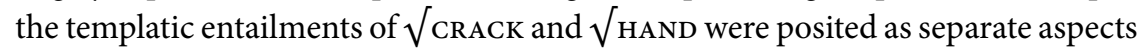


of their meanings, conjoined with the state and manner respectively that they also

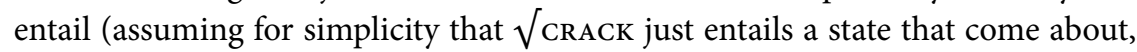
ignoring possible causation in its meaning):

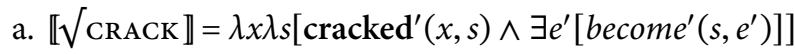
b. $\llbracket \sqrt{ }$ HAND $\rrbracket=\lambda P \lambda x \lambda v\left[\operatorname{handing}^{\prime}(v) \wedge \exists e\left[\operatorname{cause}^{\prime}(v, e) \wedge \exists s\left[\right.\right.\right.$ become $^{\prime}(e, s) \wedge$ $\operatorname{have}^{\prime}\left(\operatorname{th}^{\prime}(P), x\right.$, init $\left.^{\prime}(e)\right) \wedge a t^{\prime}\left(\operatorname{th}^{\prime}(P), x\right.$, init $\left.(e)\right) \wedge \exists z\left[a t^{\prime}\left(t^{\prime}(P), z, s\right) \wedge\right.$ $z \neq x] \wedge \neg \operatorname{have}^{\prime}\left(\operatorname{th}^{\prime}(P), x, s\right) \wedge \operatorname{have}^{\prime}\left(\operatorname{th}^{\prime}(P), \operatorname{rg}^{\prime}(P), s\right) \wedge$ $\left.\left.\left.a t^{\prime}\left(t^{\prime}(P), r g^{\prime}(P), s\right)\right]\right]\right] \quad(=(37)$ in $\$ 2.4 .1$ and $(49)$ in $\$ 3.6 .1)$

However, although we have shown that technically neither Bifurcation nor Manner/Result Complementarity hold as absolute constraints on root meanings, there are still numerous root classes that seem to obey both of them, including changeof-state verbs based on Property Concept (PC) roots in the case of Bifurcation and pure action and pure result verbs in the case of Manner/Result Complementarity. The degree to which significant classes of verbs do obey both principles, it is easy to see a functional motivation for this.

In particular, languages provide explicit means for introducing templatic meanings in conjunction with roots, namely templatic heads, and also ways of combining manner and result into a single predicate, such as resultative constructions. The existence of these compositional operations provides for the possibility of a streamlined lexicon: roots lexicalize just states and actions, and more complex meanings are built from independently motivated syntactic operations. Were there no such processes there would need to be a separate root for every possible more complex meaning. For example, if there are $n$ simple states in a language and four major event types they can be found in (e.g. simple stative, inchoative, causative, and result state) then a complete paradigm for a given state based solely on root lexicalizations would require $4 * n$ lexicalized roots, as opposed to just $n$ roots and four constructions. Similarly, if there are $n$ states and $m$ manners, we might expect some fairly large number between 1 and $n * m$ lexicalized manner+result roots to capture the variety of possible combinations of actions and manners that might correspond to possible events, as opposed to just $n$ state roots and $m$ manner roots and a few compositional processes for combining them.

So one can imagine a functional justification for principles like Bifurcation and Manner/Result Complementarity in producing a reduced set of possible root meanings. So why do complex root meanings exist? Crucially, while there might be functional value in a more streamlined lexicon of the sort Bifurcation and Manner/Result Complementarity would predict, there are other factors that push the lexicon in the opposite direction. We tentatively offer two such explanations for the existence of roots with more complex meanings, one that relies on the 
way people may conceive of certain types of states and actions, and another that relies on the role conventionalization may play in applying functional pressure to lexicalize more complex meanings.

\subsubsection{Networks of lexical entailments}

The first factor that we suggest favors roots that violate Bifurcation and Manner/Result Complementarity has to do with the ways humans conceive of certain states and actions in the world. In particular, it is easy to believe that certain types of states-such as states of flatness, length, and width-arise naturally in the world as inherent properties of something, with no prior change. But there may also be certain states of the world, such as a state of being cracked, where the very nature of the state itself - a fissure that requires change across at least space and possibly also time-simply cannot be conceived of without admitting change as a part of what defines the state. Any root that lexically entails a flatness state thus will not necessarily also have change as a part of its meaning. But any root that lexically entails a cracked state will of necessity have change as part of its meaning since that is part of the definition of the state independent of the linguistic representation.

Similarly, certain types of actions such as jogging do not necessarily require any particular change, while a handing action might be conceived of as having change as an inherent part of the action itself. Thus a root lexically entailing a jogging would not have change in its meaning. But a root lexically entailing a handing would introduce change on its own because that is part of how the action is defined. To put it another way, as mentioned already in $\$ 2.4 .1$, an alternative representation for (1) might be as in (2), where the contributions of the roots in the relevant event structures are just idiosyncratic meanings, but those meanings are associated with meaning postulates spelling out templatic aspects of their meaning:

a. $\llbracket \sqrt{ }$ CRACK $\rrbracket=\lambda x \lambda s\left[\operatorname{cracked}^{\prime}(x, s)\right]$ where for all $x, s,\left[\operatorname{cracked}^{\prime}(x, s) \rightarrow \exists e^{\prime}\left[\operatorname{become}^{\prime}\left(s, e^{\prime}\right)\right]\right]$

b. $\llbracket \sqrt{\text { HAND }} \rrbracket=\lambda P \lambda x \lambda v\left[\right.$ handing $\left.^{\prime}(x, v, P)\right]$ where for all $P, x, v,\left[\right.$ handing $^{\prime}(x, v, P) \rightarrow \exists e\left[\operatorname{cause}^{\prime}(v, e) \wedge\right.$ $\exists s\left[\operatorname{become}^{\prime}(e, s) \wedge \operatorname{have}^{\prime}\left(\operatorname{th}^{\prime}(P), x, \operatorname{init}^{\prime}(e)\right) \wedge a t^{\prime}\left(\operatorname{th}^{\prime}(P), x\right.\right.$, init $\left.^{\prime}(e)\right) \wedge$ $\exists z\left[a t^{\prime}\left(t^{\prime}(P), z, s\right) \wedge z \neq x\right] \wedge \neg \operatorname{have}^{\prime}\left(t^{\prime}(P), x, s\right) \wedge \operatorname{have}^{\prime}\left(t^{\prime}(P), \operatorname{rg}^{\prime}(P), s\right) \wedge$ $\left.\left.\left.a t^{\prime}\left(h^{\prime}(P), r g^{\prime}(P), s\right)\right]\right]\right]$

In this way, the definitions of the idiosyncratic lexical semantic primitives give rise to violations of Bifurcation and Manner/Result Complementarity because our 
understanding of certain states and actions themselves is that they involve the complex truth conditions embodied in the meaning postulates.

But the sorts of logical representations we have used so far are of course abbreviated and sometimes incomplete characterizations of whatever particular linguistically significant truth conditions are relevant for the task at hand. They do not necessarily fully represent the entire set of truth conditions that ultimately comprise the meanings of the particular lexical items. Furthermore, the meaning postulates in (2) seem stipulative. To better draw out the intuition we suggest here and why this would arise, we might consider an alternative (though semantically equivalent) way of representing the full truth conditions of a lexical item in particular as sets of lexical entailments following Dowty (1989), Dowty (1991), Primus (1999), Ackerman and Moore (2001), and Beavers (2010). In particular, building on Dowty (1989: 76-7) (see also \$1.2), lexical entailments are not all of the same degree of specificity.

As Dowty argues, the sets of lexical entailments that define thematic roles can range from highly idiosyncratic sets of quite specific lexical entailments (defining his verb-specific individual thematic roles) to very general sets of lexical entailments that are subsets of and thus shared by the individual thematic roles that are assigned by large sets of verb classes (defining his thematic role types). For example, every caused change-of-state verb defines a different individual thematic role for its object (a breakee for break, a flattenee for flatten, etc.). But the intersection of all of these filters out the idiosyncratic meanings particular to given verbs and leaves behind some highly general set of lexical entailments that we might call those associated with being a patient (presumably a subset of those that Dowty 1991: 572, (28) or Beavers 2010: 852, (86) defines as proto-patient lexical entailments). From this it in turn follows that the lexical entailments may themselves fall into two types, namely those that are general enough to be shared across (a sufficiently large number of) individual thematic roles for different verbs and those that are not.

Overlaying now the distinction between roots and templates assumed in event structural approaches, definitionally the lexical entailments associated with templatic heads must be shared across a broad set of verbs and thus be of a quite general, and also eventive, nature. The entailments associated with roots must instead include at least some highly idiosyncratic entailments not shared with templates (or presumably with too many other roots, since these lexical entailments will distinguish different roots that may occur within the same templates from one another). We can thus distinguish lexical entailments that are of the sort that are characteristically associated with templatic heads and those that are characteristically associated with roots as follows:

(3) a. Template lexical entailments - general eventive lexical entailments introduced by templatic heads (e.g. about change) 
b. Root lexical entailments - idiosyncratic lexical entailments introduced by roots and not templatic heads (e.g. names of specific, real world states and actions)

Thinking of template and root meanings this way opens up an avenue for understanding how the subpieces of verbal meaning may fit together. The lexical entailments in a given expression's meaning are not always independent of one another, but may be semantically related to one another, in particular by entailment. Within the meaning of a single templatic head or root a more specific lexical entailment can entail a more general one, so that the set of lexical entailments that comprises its meaning is not just a list of unconnected entailments but is instead a complex network of related entailments.

That entailment relations between lexical entailments are linguistically significant was already proposed by Beavers (2010), who argues that entailments of increasing specificity figure heavily into constraints on the meanings of certain classes of argument alternations. For example, among ditransitive verbs of caused possession $\mathrm{P}_{\text {have }}$ and $\mathrm{P}_{\text {loc }}$ entail have' and $R^{\prime}$ (having or co-location) respectively (see \$3.5). But have' entails $R^{\prime}$. Assuming that sets of lexical entailments are closed under entailment-that if one lexical entailment in the meaning of some morpheme entails another then both are lexical entailments of that morphemethen $R^{\prime}$ is also a lexical entailment of indirect object templates. We notate this as in (4), where 【 \ maps a morpheme to a set of lexical entailments and an arrow linking two lexical entailments within that set indicates that the one entails the other (applied to the same arguments, not notated here; the arrows are meant purely as visual aids to exemplify the idea of networks of entailments, and similarly for (5) and (8)).

(4) a. $\llbracket \mathrm{P}_{\text {loc }} \rrbracket:=\left\{\ldots, R^{\prime}, \ldots\right\}$

b. $\llbracket \mathrm{P}_{\text {have }} \rrbracket:=\left\{\ldots\right.$, have $\left.^{\prime}, \rightarrow R^{\prime}, \ldots\right\}$

The result then is that $\llbracket \mathrm{P}_{\text {loc }} \rrbracket \subset \llbracket \mathrm{P}_{\text {have }} \rrbracket$. This directly predicts the fact that to templates occur in a strictly wider set of contexts than indirect object templates, or rather that for any ditransitive root with any given set of arguments, the indirect object variant always entails the to variant but not vice versa, as per $\$ 3.3 .3$ (see Pesetsky 1995: 141, Rappaport Hovav and Levin 2008: 150-60, and Beavers 2010: 854-5, but contra Harley 2003: 41-2).

Beavers's observation is that similar entailment relations obtain between various other types of direct/oblique argument alternations (such as locative, conative, and preposition drop alternations), and that in general the relative specificity of thematic roles that arises from entailment relations between lexical entailments is key to explaining the entailment relations between alternants. This observation 
already motivates that sets of lexical entailments are structured by entailment. ${ }^{1}$ We suggest here that what is already known about entailment relations between lexical entailments derive that some roots should show violations of Bifurcation and Manner/Result Complementarity.

Specifically, the high degree of specificity of root entailments may be such that they necessarily entail the more general templatic entailments, which thus must also be part of the root's lexical entailments. For example, $\sqrt{\text { Toss describes a }}$ certain type of relatively low-energy, somewhat casual (overhand) ballistics, while

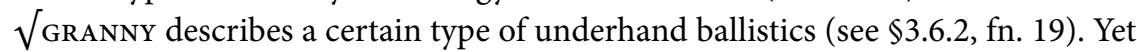
the very definition of ballistics (as we conceive of it) necessarily includes the more general releasing semantics that defined the larger class of release roots. Releasing in turn is rooted in the notion of change, and is what ensured telicity for these roots. Ballistics also requires possible co-location at some new places (whatever the overtly expressed goal is), the entailment that linked these roots to the to template but not the indirect object template and predicting root sensitivity. Thus the sets of lexical entailments that define these roots' meanings are not unstructured, but form a network, exemplified in (5), deriving entailments ensuring root sensitivity and root telicity from the more idiosyncratic meanings, without stipulation:

a. $\llbracket \sqrt{ }$ TOSs $\rrbracket:=$

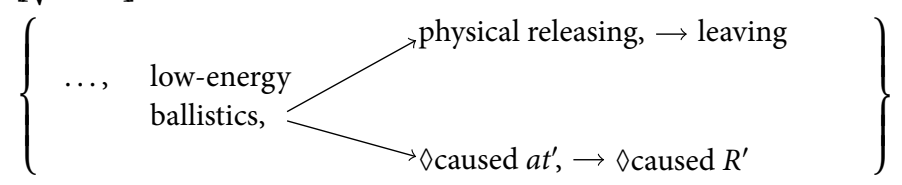

b. $\llbracket \sqrt{\text { GRANNY } \rrbracket:=}$

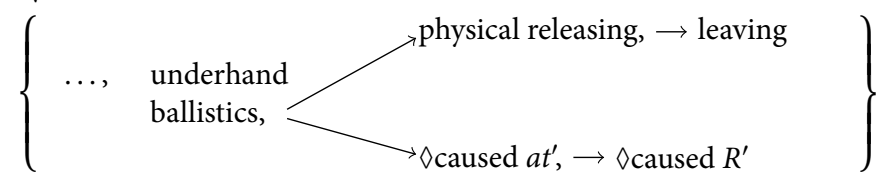

To put it another way, one cannot spell out the full set of lexical entailments that define certain kinds of idiosyncratic meanings without ultimately including by virtue of those entailments more basic templatic notions such as change or causation. ${ }^{2}$ Thus violations of Bifurcation follow by necessity.

\footnotetext{
${ }^{1}$ See also Beavers (2006: 36-44) on how entailment relations between lexical entailments can provide a link between the more idiosyncratic and broader entailments contained within a given individual thematic role in the sense of Dowty (1989).

${ }^{2}$ This idea is reminiscent of Langacker (2005: 138, 143-55), working within Cognitive Grammar, who suggests that lexical items may in many cases subsume and thus presumably entail the meanings of the constructions in which they occur in ways that explain why certain items occur in certain constructions at all and why some verb+construction combinations are more novel than others depending on whether the verb entails the constructional meaning or the combination is instead an extension (see also Iwata 2005a,b, 2008). It is not clear how the root vs. template distinction would play out in Langacker's framework, though given that, like Iwata, Langacker assumes rich lexical meanings
} 
The chain of inferences that gets from the idiosyncratic lexical entailments to the templatic lexical entailments also groups roots together into subclasses, as we have seen particularly clearly in $\$ 3.6$. For example, the specific subtypes of ballistics discussed in $\$ 3.6 .2$ all entail releasing, grouping these roots together into a group to the exclusion of (say) the roots of true possession in $\$ 3.6 .1$, which all define different ways that someone can be caused to come to actually possess something. This idea is reminiscent of the bi-partite analysis of root meanings of Arad (2005: 98, (90)) in her analysis of Hebrew morphological root/template distinction. She suggests that roots are associated with two types of meanings, the lexical class and their pure idiosyncrasy:

(6) Minimal semantic content of the root:

$\{$ lexical class + idiosyncrasy $\}$

The lexical class for Arad includes medium-grained lexical entailments like adding/subtracting or motion from a source (e.g. Pinker 1989's narrow range classes or those of Levin 1993) that limit what templates a root can occur in. The idiosyncrasy determines what unique contribution the root makes distinct from others in the same lexical class.

We suggest that lexical class may in some cases be defined by medium grain lexical entailments that are entailed by the more idiosyncratic root entailments and need not be stated separately. Templatic entailments may in turn be entailed by the lexical class entailments. For ditransitives we ultimately observed four major types of roots in a classification that exemplifies this (simplifying Beavers 2011a: 44, Fig. 1, ignoring durativity):

\begin{tabular}{|c|c|c|}
\hline \multicolumn{3}{|c|}{ Specificity of Lexical Entailments per Root } \\
\hline Root & Root Class & Templatic \\
\hline Entailments & Entailments & Entailments \\
\hline Types of giving & True possession & have', $R^{\prime}$ \\
\hline Types of obliging to give & Future having & have', $R^{\prime}$ \\
\hline Types of accomp. motion & $\overline{\text { Arrival }}-\overline{-}$ & $R^{\prime}--$ \\
\hline Types of releasing & Release & $R^{\prime}$ \\
\hline
\end{tabular}

The roots that are specific enough to ensure that actual or prospective having are part of their meaning (the ones above the dashed line) are the ones that show no contrast in the dative alternation, while those that do not (the ones below the dashed line) do show a contrast. This derives root sensitivity through a series of entailment relations between the lexical entailments of the root and those of the templates they occur in. 
Turning now to change-of-state verbs, as noted above some types of states may be conceived of as only arising through a change. These are the meanings that would be described by roots that would thus necessarily have change as part of their meaning. This predicts the existence of at least a subset of the class of result roots from Chapter 2 . Thus a root like $\sqrt{\mathrm{CRACK}}$ inherently describes a state where some kind of otherwise continuous integrity fails to obtain at some point on the spatial extent of an entity (i.e. minimally a spatial extent change, if not always a temporal change):

$$
\begin{aligned}
& \llbracket \sqrt{ } \text { CRACK } \rrbracket= \\
& \left\{\ldots, \text { has fissure in material integrity, } \rightarrow \text { integrity changes, } \rightarrow \text { become }^{\prime} \ldots\right\}
\end{aligned}
$$

Other roots of damage (e.g. $\sqrt{\text { SHATTER, }} \sqrt{\text { DESTROY }}$ ) share the change in integrity entailment but differ in the idiosyncratic nature of the change in integrity, defining a lexical class. Thus it is not unexpected that some stative roots will have change entailments as a part (and extending the argument also causation entailments if a given state could only have been caused).

Of course, it does not follow that roots must include templatic entailments in their set of lexical entailments. For example, a stative root like $\sqrt{\text { FLAT does not }}$ entail templatic entailments, as discussed in Chapter 2, evidenced by the lack of change inferences with the simple adjective flat. Rather, any cause and change found in its verbal uses come from the template. Similarly, a manner root like $\sqrt{\text { JOG }}$ need not entail any type of state change, either. Any state change that ever does arise with such a root (e.g. John jogged to the store) must come from the template or some other lexical item. That said, there are conditions on which templatic meanings may be "bolted on" - in the terms of a reviewer-to a given root. Some unergative motion verbs causativize, as in walk the dog, but others do not, as in \#swim the dog. We do not make any claims about what governs such processes. Rather, our claim here is that in some cases, owing to a root's idiosyncratic content it could of necessity have templatic meaning in it owing to the idiosyncratic action or state it describes (see also e.g. Levin and Rapoport 1988; Goldberg 1995: Ch.2).

Furthermore, just as some templatic entailments may entail others (as with the have' relation entailing the $R^{\prime}$ relation above), root entailments may also entail other root entailments, something that would explain at least some cases of Manner/Result Complementarity violations. As noted above, it is the manner of ballistic motion that gives rise to the inference of releasing that is the specific, idiosyncratic result state of that class of roots. Thus there is an inherent connection between the manner and the result in these cases. Conversely, some types of states may by definition only arise from certain sorts of very specific processes. The manner of cooking verbs we discussed in Chapter 4 may be like this. As the types of contexts we established to show that such verbs lack restitutive readings $\$ 4.5 .2$ make clear, it is quite difficult if not impossible to separate out the specific result states of verbs like braise and fry that would be distinct from the particular processes that give rise to them. The degree to which having brown, fatty edges is 
part of the outcome of frying it is not just any case of having brown, fatty edges. It is the specific version of that state that arises from a particular type of application of heat. Similar observations have been made by Beavers and Koontz-Garboden (2017a: 869-70) about changes that conventionally arise as outcomes of particular manners entailed by certain motion verbs.

Thus entailment relations between lexical entailments will give rise inescapably to violations of both Bifurcation and Manner/Result Complementarity. To return to the meanings we posited in (1), the overtly represented templatic conditions may be superfluous since the lexical entailments of the idiosyncratic information already has it as part of the definitions. Alternatively, the meaning postulates in (2) simply spell out in formal representational terms the entailment relations between lexical entailments, i.e. the basic truth conditions of cracked' and hand'. Conversely, templatic lexical entailments do not entail root entailments, since specificity runs in only one direction, though some templates may be more specific than others (as with the relationship of the two P heads of ditransitive templates). Thus entailment relations from roots to templates and other roots should just run in one direction. In sum, overlap in root and template meaning is not just attested but expected if roots entail such specific information.

\subsubsection{Lexicalized conventional associations}

While entailment relations may explain some of the data, they cannot explain all of it. For example, some change-of-state verbs describe outcomes that in principle should be divorcible from a change, yet seem not to be. An example of this is melt (as pointed out to us by Malka Rappaport Hovav, p.c.). The result state of melt is presumably to be in a liquid state, or at least a state that has some relatively high degree of viscosity even if not technically a liquid, though we assume the state is being a liquid here for simplicity. It is furthermore easy to imagine that this state can hold of something without a change occurring, if the given substance is always a liquid.

Nonetheless, as Rappaport Hovav (2010: 7) shows, melt does not allow a restitutive reading with again (as with (47) in $\$ 2.4 .1$, repeated here), nor does it have a simple adjectival form, patterning thus as a result root:

(9) [ An ice cream factory manufactures ice cream from a package of ingredients by adding water and then freezing the result. After adding the contents of the package to water and freezing it, Kim lets it melt into a liquid state. ]

\#Kim melted the ice cream again.

(necessarily two defrostings)

Conversely, liquefy shares more or less the same resulting state, but does allow restitutive modification: 
(10) As far as I know, petroleum is a liquid. So according to the plaque (and my mind), they took a liquid, gasified it and then liquefied it again. :? [sic] <http: //www.thescienceforum.com/chemistry/8136-liquefied-petroleum-gas. html > (Accessed February 22 2018)

This shows that the state shared by these two verbs is not one that requires a change. But if this is the case, why does melt pattern as a result root?

Here we suggest there is another avenue by which Bifurcation and Manner/ Result Complementarity may be violated. The correlation of that state with a change is for whatever reason common or conventional enough that it has been lexicalized into a single root as a packaged event category type. In particular, through daily interactions with cooking and climate it is a common association between something becoming hot or having heat applied to it and the thing thus becoming liquid that a stative root has come to have a meaning describing this one specific larger event type, even if there is no necessary connection with that change and that state. Even the paradigm case of ice melting when being left out (pointed out to us by a reviewer) is one that involves a specific set of atmospheric conditions, though no particular process is involved and the atmospheric conditions are the "normal" ones of ambient room temperature. This again motivates a root that entails both a liquid state and some change into that state. However, this is not the only way that something can be a liquid, and hence liquefy lexicalizes the same state but via a PC root lacking the entailment of change. So this provides a potential second avenue by which violations of Bifurcation may arise.

We may see similar effects with Manner/Result Complementarity violating verbs. For example, for some speakers (ourselves included) crucify requires not just the change that the patient be attached to a cross and hoisted in the air, but the further change of death. Yet death need not require the particular manner entailed by crucify, and the particular manner entailed by crucify need not necessarily yield death. However, it could be that the common conventional association of that manner with that result in relevant contexts of usage is such that English has lexicalized a root for some speakers for exactly that scenario. Thus in addition to cases where a given basic event type is part and parcel of a given action or state, and cases where a given action or state is defined solely in term of a given state or action respectively, common conventional associations of different types of actions and states may lead to lexicalization of roots with more complex meanings.

\subsubsection{Summary}

In sum, the deeper explanation for why roots entail complex meanings has to do with the relationship between templatic and root lexical entailments and also different root entailments. We have suggested that template and root meanings 
differ not ontologically (contra Arad 2005; Embick 2009) but in specificity. Template meanings consist of general eventive lexical entailments, while root meanings include idiosyncratic lexical entailments. The latter may entail the former, and thus they will also be included in root meanings. Thus sometimes templates augment the root, but sometimes the root entails the template meaning, subsuming it completely or augmenting it (see e.g. the various proposed connections of verb and construction meaning summarized in Goldberg 1995: 65). Similarly, root entailments may entail other root entailments, giving rise to violations of Manner/Result Complementarity. Certain violations of Bifurcation and Manner/Result Complementarity thus follow as a necessity from lexicalizing certain meanings. Conversely, they may instead arise from lexicalization of common or conventional cooccurrences that get lexicalized into a single root.

Putting these together, the apparent functional motivations for a lexicon wholly constrained by Bifurcation and Manner/Result Complementarity are balanced against competing factors that result in violations of these principles. The result will be something like Bifurcation and Manner/Result Complementarity as defaults, with particularized exceptions arising for principled reasons. The next question is what the conclusions we have reached here say about possible root meanings and thus possible verb meanings, in the sense of what truth conditional content a verb can actually have.

\subsection{Are there any constraints on root meaning?}

Bifurcation and Manner/Result Complementarity are two of the best supported claims in the literature for constraints on root meaning, though we have claimed that they do not hold. Of course, these are just two possible constraints on root meaning we might consider. Other conditions are possible, and would require additional work to fully evaluate. For example, Dowty (1979: 125-9), in his discussion of the importance of carefully studying what we are here calling root meaning, sketches a few tentative proposals, though the discussion above already calls these into question.

One constraint Dowty proposes is that (translating his proposals into the terminology used here) no root will describe values along different scales simultaneously. However, we have seen already that this cannot be the case. In our study of ditransitives we noted that some roots entail changes in location and in possession simultaneously, including for example hand. Beavers (2011a: 27-30) argues that these two changes necessarily represent two concurrent changes at once that are not reducible to one another, although the ultimate analysis conflates the two changes onto a single scale. A second constraint Dowty suggests is that no root will encode discontinuous scalar values. The motivation for this might be that scales are independently defined objects and that if a root describes a scale or 
state on a scale it must pick out the entire scale. However, some scales that might in some contexts involve continuous values may in other contexts be perceived of as having discrete, non-continuous values (Beavers 2008: 251-2). Thus The image on the screen grew could describe continuous increase in size or a single jump from (say) small to large in one instance. Alternatively, perhaps the scale found in the root of grow is underspecified for gradability and Dowty's claim only specifically pertains to roots describing discontinuous changes along scales where the scale is definitively not underspecified. Whether this is true or not would require more exploration, but at least at the moment it is not obvious prima facie if a constraint such as this does limit possible root meanings.

Finally, Dowty proposes that no root will entail different states at different times, i.e. there will be nothing like the famous grue of Goodman (1954: 74-82) meaning "green until time $t$ and blue thereafter." Taken in the most literal sense intended by Goodman of $t$ being some quite specific time (e.g. September 10,1953), we concur that this is unlikely to be a possible root meaning. However, as Dowty suggests this is likely not about roots per se but more generally about verb meanings overall (see below). Alternatively, if the constraint is against a root naming different states holding at different non-specific times, from our case studies above we do see root meanings that are grue-like in this sense. In particular, change-of-state verbs can have stative roots that describe changes, and thus two states at different, albeit non-

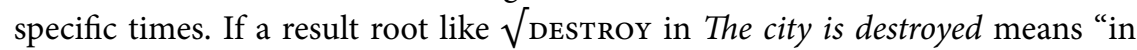
a state of holding the maximal degree of destruction that arose from a process changing it from some degree of destruction below the maximum," this is exactly the sort of meaning that should be banned.

Of course, there may be ways in which such a constraint does pan out as

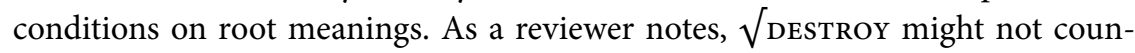
terexemplify a ban on roots naming two scalar states at different times if the prior state of not being destroyed is presupposed rather than asserted (assuming Dowty's proscription was against two states being asserted). Alternatively, one might interpret Dowty's claim as being about changes between distinct but fully specified states along a scale. In destroy the degree of destruction of the final state is specified but the degree of destruction of the initial state could be any degree on the gradable scale of destruction. In other words, it is some existentially quantified degree defined simply as "not maximally destroyed," which is not a specific degree. However, for non-gradable scales as with break or die there are only two degrees to which the property can hold: the maximal or the minimal degree. But since each is fully defined and not holding one degree means definitely holding the other, existential quantification over the initial degree will still result in a meaning where the initial state was specific. To put it another way, on a non-gradable scale "holds a degree that is not the maximal degree" and "holds the minimal degree" are truth conditionally equivalent. Beavers (2013: 694-5) makes an analogous point in a proposal for why achievements do not undergo the conative alternation (e.g. 
Mary broke (*at) the dishes). Beavers proposes that the conative for verbs that entail quantized change reflects existential quantification over the final state on the scale, but for achievements this would be a vacuous operation since the final state would still be fully determined as the only state that is not the initial state, and thus the alternation is blocked.

Thus absent more spelled out conditions like the ones Dowty suggests, it does seem that there may well not be any particular conditions on what roots can or cannot mean. Thus it is unlikely that event structural approaches really have much unique to say about possible and impossible verbs truth conditionally, at least as arising from conditions on roots. This is not to say there can be no constraints on word meaning at all. For example, in $\$ 4.2 .3$ we mentioned that change-of-state predicates may not describe changes along two wholly distinct scales (Goldberg's 1995: 81-88 Unique Path Constraint), even if two changes along the same scale or related scales are allowed. Thus verbs like hand perhaps arise from a natural semantic affinity between change in possession and change of location (see Beavers and Koontz-Garboden 2017a: 868-70 for more on what kinds of changes might go together). Furthermore, naturalness constraints may make some verb meanings impossible. We are unlikely to find roots and thus words that describe extremely implausible eventualities, and indeed a meaning like grue assuming a specific time point $t$ strikes us as unlikely on these grounds. But these sorts of constraints are not really conditions on root meanings in particular. Rather, they seem to be more general conditions on what might count as a plausible or natural event. Thus we might indeed expect to find them applying just as well to the larger event structures of single verbs (as with Dowty's prohibition on reference to specific times in event structural formulae in general) and even complex, derived verbal predicates such as resultatives. Indeed, resultatives were in fact a key data point in the development of the Unique Path Constraint. Thus templates as well as roots are probably subject to conditions such as these. If so, nothing specifically about roots per se seems to make predictions about what a verb can mean. But if roots themselves (and maybe even event templates) do not rule in or out possible verb meanings, what do they do?

\subsection{Possible and impossible verbs}

Although we are skeptical that event structures place special constraints on impossible verb meanings, at least as determined by roots, this is not to say that our ultimate conclusions here are wholly negative. Rather, we believe there are significant positive findings that emerge from having shown that Bifurcation and Manner/Result Complementarity do not hold. In particular, we can view roots not in terms of what lexical entailments they hold in some general sense, but specifically in terms of templatic and root lexical entailments as defined in (3). 
In this section we summarize our major findings in this regard and show that it produces a typology of root classes that, when overlaid onto the preexisting theory of templates we have assumed here, produces a predictive theory of verb classes regarding lexical entailment patterns and grammatical behavior, albeit one that must take root class into account as well as template class.

\subsubsection{A typology of roots}

We first consider what ingredients we have suggested so far that form the basis for classifying root meanings-which templatic and root lexical entailments it has-plus the position in which the root occurs. For simplicity we notate the relevant entailments in terms of the following features (which are just meant to be descriptive, informal mnemonics for the classes of meanings discussed throughout the book; they have no actual theoretical status):

(11) a. \pm state - the root does/does not describe a state

b. \pm manner - the root does/does not describe an action

c. \pm result - the root does/does not entail a change

d. \pm cause - the root does/does not entail causation

We assume for semantic reasons that there are certain collocational restrictions on lexical entailments. In particular, we assume that being + result entails being +state, since become $e^{\prime}$ entails something that has come about. We also assume being + cause entails being + result, since cause entails that there is a caused event. These are not root-specific stipulations. They in fact mirror standard conditions on possible event templates (see e.g. Rappaport Hovav and Levin 1998: 108, though whether these follow from conditions on a theory of event templates in particular that also apply to roots or from more basic constraints on possible or natural events applicable to both we leave open). Finally, we ignore any roots that are not positively defined for at least one of these features. We do not suggest that such roots do not exist, e.g. they would include individual-naming roots as per Rappaport Hovav and Levin (1998), Harley (2005), and Levinson (2007). But roots such as these are outside the scope of this study. From these features the roots we have discussed in this book fill out what we believe is a fairly exhaustive typology, with certain apparent gaps in the typology predicted by functional and sometimes formal considerations.

First, something positively specified only as +state or + manner would constitute the roots of canonical change-of-state or action verbs respectively-PC roots of change-of-state verbs like $\sqrt{\text { FLAT }}$ or the roots of pure action verbs such as manner-

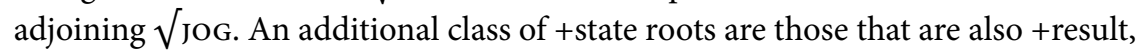
but nothing else. These would be basic result roots that describe states that must 
have come about (as per $\$ \$ 2.2-2.4$ ), but not that the event was caused in any way. Of course, there is also a class of +state, +result roots that are furthermore + cause, but not +manner. These would be roots that entail caused changes into the described state, but not necessarily any particular manner that leads to them. In Chapter 2 we did not make a particular distinction between roots entailing changes that are caused vs. those that are not (and in fact treated all result roots in that chapter as entailing change but not causation). But if we accept that the roots should be subdivided according to this, it might be what underlies the distinction between change-of-state verbs that have been argued in prior literature to generally be lexicalized crosslinguistically as basic causatives vs. those that are not. In particular, this could distinguish Levin and Rappaport Hovav's (1995: 90-101) externally caused change-of-state verbs like break, which they argue are lexically causative in many languages, and internally caused change-of-state verbs like blossom, which are lexically inchoative in many languages (see e.g. KoontzGarboden 2009a: 108-10 for an explicit argument that the inchoative variants of verbs based on roots with these meanings in Spanish are distinguished exactly in the presence vs. absence of causation).

Finally, though, there could be caused change-of-state roots that are positively specified as +manner as well, i.e. are positively specified for all of the relevant lexical entailment types. These of course would be the Manner/Result Complementarity-defying roots such as $\sqrt{\text { DROWN }}$ or $\sqrt{\text { HAND, which differ from }}$ one another in whether they sit in complement position or adjoined position within the event structure, but in either case deriving verbs that have the semantics of resultatives (but not the full syntax; see $\$ 4.5$ ).

The full typology generated by these features is given in (12), with attested root types filled in by examples:

\begin{tabular}{|c||c|c|}
\hline \multicolumn{1}{|c||}{ root entailments } & \multicolumn{2}{c|}{ root position } \\
\cline { 2 - 3 } & adjoined & complement \\
\hline \hline -manner, -cause, -result, +state & & $\sqrt{\text { FLAT }}$ \\
\hline -manner, -cause, +result, +state & & $\sqrt{\text { BLOSSOM }}$ \\
\hline -manner, +cause, +result, +state & & $\sqrt{\text { CRACK }}$ \\
\hline +manner, -cause, -result, -state & $\sqrt{\text { JOG }}$ & \\
\hline +manner, -cause, -result, +state & & \\
\hline +manner, -cause, +result, +state & & \\
\hline +manner, +cause, +result, +state & $\sqrt{\text { HAND }}$ & $\sqrt{\text { DROWN }}$ \\
\hline
\end{tabular}

While it is clear how the roots we have discussed fit into the typology, there are gaps in (12) that require explanation. Are these predicted but to date unattested, or are there principled reasons why these did not emerge in our study? We suggest 
that these gaps all correspond to root types that are indeed ruled out or at least are heavily dispreferred for principled reasons.

First, two broad classes-roots sitting in adjoined position but that are -manner (regardless of what else is specified) and roots sitting in complement position but that are -state (which in this case would be pure manner roots) - are as far as we know largely unattested. These constitute four of the unattested roots in (12), the empty cells in the first four rows. Technically speaking, such roots are not impossible in any compositional sense. We outlined in $\$ 3.5$ an analysis allowing an adjoined root to contribute a state (in addition to a manner) and in $\$ 4.5 .3$ an analysis allowing a complement root to contribute manner (in addition to a state). Thus there is no prohibition from a result state coming from an adjoined root or a manner coming from a complement to a $v_{\text {become }}$. One possible case where we have seen something like this is $\sqrt{\text { GIVE }}$ in $\$ 3.6 .1$, where the supposed giving manner may have few if any conditions on it at all, though this is the only root we have discussed like this.

Significantly, if such roots are ultimately attested as a major class among intransitives, they would essentially derive syntactically unergative verbs with pure change-of-state meanings and syntactically unaccusative verbs with pure action meanings, assuming that in general the syntax of intransitive verbs with adjoined roots is unergative and with complement roots is unaccusative. This would, however, constitute a mismatch of the canonical associations of verbs with those meanings and the syntactic position of the roots. Typical unaccusatives entail just change of state and typical unergatives entail just action. We conjecture that adjoining roots for pure change-of-state meanings and complement roots for activity meanings are likely to be dispreferred on simple naturalness grounds. The resulting verb meanings would be truth conditionally identical to equivalent verbs derived from non-"mismatched" roots, but would require more complex denotations that involve non-local constraints. Thus on markedness grounds we might expect such roots to be relatively rare. Indeed, $\sqrt{\text { GIVE }}$ is the only root we examined that might have such a meaning, though further investigation is required.

Of course there are also roots that describe both a state and an action simultaneously, namely Manner/Result Complementarity-defying roots. In these cases we have the opposite problem: it is not obvious which position would be more natural for them. Hence we would expect such roots to split along the lines of which position they canonically occur in, and this is precisely what we observe in the difference between $\sqrt{\text { HAND }}$ and $\sqrt{\text { DROWN }}$ respectively. Indeed, given the constraints on single rootedness in event structures, roots such as these would be the only way for a single verb to have both manner and result entailments in it simultaneously, thus motivating the existence of these type of roots on functional grounds. A question for future crosslinguistic research is whether the link between 
syntactic position and event type is random or not. Based on what we have said here, we have no reason to believe that Manner/Result Complementaritydefying roots like $\sqrt{\text { HAND }}$ should always be adjoined high or $\sqrt{\text { DROWN should }}$ always be a low complement. If it turns out that roots with meanings like English $\sqrt{\text { HAND }}$ are always adjoined and roots with meanings like $\sqrt{\text { DROWN }}$ are always complements crosslinguistically, then there will be more to say. Future research should investigate these empirical and theoretical questions.

But what about the class of roots that describe a state and also an action but are not also positively specified for either a non-caused or caused change, i.e. the two entirely blank rows in the bottom half of (12)? The first of these-+manner and +state but -cause and -result-we suggest is ruled out on naturalness grounds: there would be nothing that relates the described manner and the described state together. Why the two would be described by a single root, and what kind of function would such a root fulfill in a language? The most natural connection would be causation, as discussed in $\$ 1.6 .2 .1$ and $\$ 4.6$, but then we would have +cause and +result. It is of course possible that in some language there could be some root that describes both an action and a state but with no connection between them, or there is some other connecting semantics. But it is not immediately clear what functional role such a root would have, or even what sort of event template it would be appropriate for. Thus such roots could be considered to not exist simply for not serving any obvious linguistic function. Tellingly, we are not aware of any event template with such a function, either, suggesting again that this is a broader condition.

As for the second class-+manner, +change, and +state but -cause-this might constitute cases where a patient undergoes a change in a certain manner (following on the idea that manners can sometimes apply to patients as per $\$ 4.3 .1, \mathrm{fn}$. 10 and that changes of state event may have manner as per a reviewer's suggestion in \$4.5.3). One possibility would be unergative motion verbs such as intransitive slide as in Mary slid down the hill, which Beavers and Koontz-Garboden (2017a: 863) suggest have both necessary displacement (cp. jog in She jogged in place) and also a manner. However, the question is whether there is really no causation in the meaning of such verbs, or whether there is causation but it is self-caused action (as suggested by Kemmer 1993: 69-70 and Van Valin and LaPolla 1997: 111). Another possibility might be sound emission verbs in a motion context, as in The fly buzzed across the room. However, these may also have causation in their meaning, although the causal relationship is reversed from the typical resultative causal structure where here the motion may cause the buzzing (as per Goldberg 1995: 62; Verspoor 1997: 114-7, Levin 1999: 210; Goldberg and Jackendoff 2004: 541). Ultimately, the question of whether a change and a manner can exist together in a single meaning without causation is an open one.

Interestingly, the possible combinations of lexical entailments of the clearly attested roots give rise to an alternative classification of roots that mirrors every class of attested event template that could underlie any such verb. Describing a 
state or action in and of itself is not the semantics of any $v$ head, entailing a result incorporates the semantics of $v_{\text {become }}$, entailing causation incorporates the semantics of $v_{\text {cause }}$ (which requires a $v_{\text {become }}$ ), and further describing a manner for $v_{\text {cause }}$ essentially creates the semantics of $v_{\text {act }}$ (albeit through attachment to $v_{\text {cause }}$ ). The ditransitive roots we examined above all fall into this final class, but specify that the described state is one of possession or co-location (or both), thus filling out templatic stative information as part of their classification as +state roots by also entailing the meanings of $\mathrm{P}_{\text {loc }}$ or $\mathrm{P}_{\text {have }}$ plus $\mathrm{P}_{\text {loc }}$ (since the meaning of $\mathrm{P}_{\text {have }}$ entails the meaning of $\mathrm{P}_{\text {loc }}$ ). Roots with each such meaning are illustrated in (13):

\begin{tabular}{|c||c|c|}
\hline entailments & root & $\begin{array}{c}\text { templatic head } \\
\text { entailed }\end{array}$ \\
\hline \hline +manner, -cause, -result, -state & $\sqrt{\text { JOG }}$ & none \\
\hline -manner, -cause, -result, +state & $\sqrt{\text { FLAT }}$ & none \\
\hline -manner, -cause, +result, +state & $\sqrt{\text { BLOSSOM }}$ & $v_{\text {become }}$ \\
\hline manner, +cause, +result, +state & $\sqrt{\text { CRACK }}$ & $v_{\text {cause }}, v_{\text {become }}$ \\
\hline +manner, +cause, +result, +state & $\sqrt{\text { DROWN }}$ & $v_{\text {act }}, v_{\text {cause }}, v_{\text {become }}$ \\
\hline +manner, +cause, +result, +state & $\sqrt{\text { TOSS }}$ & $v_{\text {act }}, v_{\text {cause }}, v_{\text {become }}, \mathrm{P}_{\text {loc }}$ \\
\hline +manner, +cause, +result, +state & $\sqrt{\text { HAND }}$ & $v_{\text {act }}, v_{\text {cause }}, v_{\text {become }}, \mathrm{P}_{\text {loc }}, \mathrm{P}_{\text {have }}$ \\
\hline
\end{tabular}

There is no verbal event templatic meaning not found in the meaning of some root:

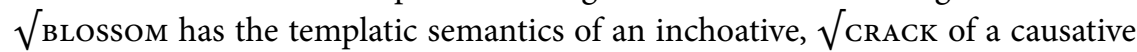
(assuming it falls in with externally-caused change of state as above), $\sqrt{\text { DROwN of }}$ a resultative (a fully specified causative change-of-state event template Rappaport Hovav and Levin 1998: 118-23 posit as only arising through augmentation of more

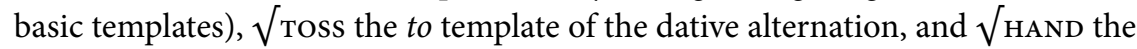
indirect object template. Furthermore, just as certain templates are impossible, roots that describe impossible templatic meanings are ruled out. Only roots corresponding to attested templates are found, though this is not a condition on roots per se but either a more general, inherited condition on event templates and/or even more general conditions on natural types of events.

In sum, the typology in (12) and its extension in (13) is as expected given reasonable assumptions about decompositions in verb meaning and semantic markedness, meaning the typology should reflect the most likely attested classes, with the gaps either rare or unattested altogether. We turn next to what this classification of roots says about verb classes.

\subsubsection{A theory of possible verb classes}

We are now finally in a position to consider what predictive theory of verb classes these results lead to. First and foremost, we reiterate a point made throughout this 
study (see $\$ 1.4 .3, \S 2.6, \$ 3.10$, and $\$ 4.7$ ) that while roots and templates may entail the same meanings, templates still have an ontologically different status in terms of defining linguistically significant structured meaning representations in ways roots do not. This justifies their existence as a part of a theory of verb meaning even if the existence of certain types of truth conditions in the meaning of a given surface verb do not.

For example, their general productivity makes predictions about morphological paradigms for roots, including regular, derivational morphology. Templates also provide the structure that guarantees an analysis of sublexical scopal modifiers and appropriate asymmetric prominence relationships between arguments. Roots that introduce exactly the same meanings as the templates that generate those grammatical facts do not themselves determine the same grammatical facts. As discussed in $\$ 3.10$ templatically introduced possession within ditransitives as with $\mathrm{P}_{\text {have }}$ ensures asymmetric binding of indirect objects into direct objects, but root introduced possession as with bequeath does not. Similarly, as discussed in $\$ 4.7$ templatically introduced causation as with $v_{\text {cause }}$ can license an effector DP but root introduced causation as with braise cannot (as when it is used in a stative template). Finally, two meanings bundled together in a single root cannot be separated by sublexical modification as with again, even when the same meanings coming from templates sometimes can be separated. This in turn was part and parcel with the notion of single-rootedness discussed in $\$ 4.5$. When roots entail multiple types of idiosyncratic meaning they do so as a single unit, ruling out a class of verbs that would correspond to true lexical resultatives.

Thus templates still make verb class predictions, mainly having to do with argument prominence, behavior of sublexical modification, and some aspects of regular derivational morphology. But once we overlay the root classes in $\$ 5.4 .1$ onto this theory of templates the possible verb classes become more complex, yet still form a constrained theory. First, the correlates of root meaning to root position discussed in $\$ 5.4 .1$ give rise to expectations about syntax. While, in principle, unergative change-of-state verbs and unaccusative activity verbs are possible, the expectation is that these should be fairly rare. Second, this root typology predicts that some effectively identical truth conditional meanings (manner+result roots) can be instantiated in two totally distinct grammatical ways, as complement roots or adjoined roots. While this does obliterate any correlation that might have been predicted between truth conditional content and syntactic properties, the two types of manner+result verbs are nonetheless predicted to otherwise pattern systematically like pure action or pure result verbs syntactically, as per $\$ 4.5$. There is no prediction of any sort of mixed grammatical properties for such verbs despite their mixed semantics. Third, given that conditions on possible event templates or event types in general seem to govern possible root meanings, we still predict that meanings that do not accord with such conditions-such as manners plus states with no change or causation-will not arise as possible verb meanings. This should 
result in a truth conditional constraint on possible verb meanings, though again it is not specific to roots per se but something more general.

Still further, we demonstrated in the preceding chapters that roots can have other direct grammatical effects on regular morphology, modification, and argument realization. In $\$ 2.4 .1$ we suggested that overt marking on English adjectival forms of the roots of change-of-state verbs can and perhaps should be conditioned directly on root semantics (modulo lexical idiosyncrasy). Similarly, we argued in $\$ 3.8$ that direct semantic incompatibility of root and template lead to a dispreference for certain roots of ditransitive verbs occurring in the indirect object template. Finally, as discussed in $\$ 3.6$ the semantic effect of argument alternations can sometimes be determined by the root, including telicity and the modifiers that probe for it, sometimes deemed a factor usually conditioned on templates (see \$3.4). These predictions were derived without postulating canonical realization rules or other conditions that constrain what templates a root may occur in that give rise to indirect effects of roots on the grammatical properties of surface verbs, as often adopted in prior theories (see \$1.3.3). These root-conditioned effects are also more in line with what templates might be expected to predict (morphology, modification, and argument realization) despite coming from the root. This means that on the root typology we have proposed the verb's total set of grammatical and semantic behaviors have to be taken in totality to deduce which root and which template are conspiring to produce those behaviors.

In sum, there are systematic classes of roots and of templates, and each comes with properties that will result in particular behaviors of the verbs formed when these roots occur with these templates. Thus the root typology we have outlined here derives predictions about possible verb classes that crosscut but also interact with predictions already made by templates.

\subsection{Concluding remarks and outlook}

In conclusion, the semantic root typology we proposed in $\$ 5.4$ is not a typology that rules out particular root meanings as a particular set of conditions on roots per se, other than whatever constraints are inherited from how event templates are constructed or other broader constraints on possible meanings. But when we overlay this typology of roots with the event structures they occur in, the combination of meanings and grammatical behaviors seen for particular roots and particular templates come together in a way that still derives predictions about verb classes. Combinations of argument realization facts, entailment patterns, regular morphological properties, and interpretations of sublexical modification, among other things, reflect specific combinations of certain types of roots with certain types of templates. 


\section{CONCLUSION}

The upshot is that while there is a lack of specific conditions on what a root can mean and thus no root-specfic constraints on what a verb can mean, providing a tentative answer to the central question Dowty (1979: 125-9) posed, there are still predictions about systematic correlates of particular verb meanings to particular syntactic properties, where roots as well as templates serve as a source of systematicity. The predictions are not what event structural approaches that assume Bifurcation and Manner/Result Complementarity would lead us to expect. Entailing change does not require $v_{\text {become }}$ syntax, nor does entailing possession yield possessor prominence over possessees, nor does entailing both a manner and a result require resultative syntax, among other things. But the proposals we have outlined do suggest a continued predictive value of event structures as part of an understanding of verbal meaning. 


\section{References}

Ackema, Peter and Maaike Schoorlemmer. 1994. The middle construction and the syntaxsemantics interface. Lingua 93: 59-90.

Ackema, Peter and Maaike Schoorlemmer. 2004. Middles. In M. Everaert and H. van Riemsdijk, eds., The Blackwell Companion To Syntax, pages 131-203. Oxford: WileyBlackwell.

Ackerman, Farrell and John Moore. 1999a. Syntagmatic and paradigmatic dimensions of causee encodings. Linguistics and Philosophy 22:1-44.

Ackerman, Farrell and John Moore. 1999b. 'Telic entity' as a proto-property of lexical predicates. In M. Butt and T. H. King, eds., Proceedings of the LFG 1999 Conference, pages 1-15. Stanford, CA: CSLI Publications.

Ackerman, Farrell and John Moore. 2001. Proto-Properties and Grammatical Encoding: A Correspondence Theory of Argument Selection. Stanford, CA: CSLI Publications.

Alexiadou, Artemis and Elena Anagnostopoulou. 2004. Voice morphology in the causativeinchoative alternation: Evidence for a non-unified structural analysis of unaccusatives. In A. Alexiadou, E. Anagnostopoulou, and M. Everaert, eds., The Unaccusativity Puzzle, pages 114-36. Oxford: Oxford University Press.

Alexiadou, Artemis and Elena Anagnostopoulou. 2008. Structuring participles. In C. B. Chang and H. J. Haynie, eds., Proceedings of the 26th West Coast Conference on Formal Linguistics, pages 33-41. Somerville, MA: Cascadilla Proceedings Project.

Alexiadou, Artemis, Elena Anagnostopoulou, and Florian Schäfer. 2006. The properties of anti-causatives crosslinguistically. In M. Frascarelli, ed., Phases of Interpretation, pages 187-211. Berlin: Mouton de Gruyter.

Alexiadou, Artemis, Elena Anagnostopoulou, and Florian Schäfer. 2015. External Arguments in Transitivity Alternations: A Layering Approach. Oxford: Oxford University Press.

Arad, Maya. 2005. Roots and Patterns: Hebrew Morpho-Syntax. Dordrecht: Springer.

Arka, I Wayan. 2003. Balinese Morphosyntax: A Lexical-Functional Approach. Canberra: Pacific Linguistics, School of Pacific and Asian Studies, the Australian National University.

Arunachalam, Sudha and Anubha Kothari. 2011. An experimental study of Hindi and English perfective interpretation. Journal of South Asian Languages 4: 27-42.

Bach, Emmon. 1986. The algebra of events. Linguistics and Philosophy 9: 5-16. References to reprint in Paul Portner and Barbara H. Partee (eds.) (2002) Formal semantics: The essential readings. Oxford: Blackwell.

Baker, Mark C. 1988. Incorporation: A Theory of Grammatical Function Changing. Chicago: University of Chicago Press.

Baker, Mark C. 1997. Thematic roles and syntactic structure. In L. Haegeman, ed., Elements of Grammar, pages 73-137. Dordrecht: Kluwer.

Baker, Mark C. 2003. Lexical Categories: Verbs, Nouns, and Adjectives. Cambridge: Cambridge University Press.

Bale, Alan C. 2007. Quantifiers and verb phrases: An exploration of propositional complexity. Natural Language and Linguistic Theory 3: 447-83. 
Bar-el, Leora, Henry Davis, and Lisa Matthewson. 2005. On non-culminating accomplishments. In Proceedings of NELS 35, pages 79-92.

Barker, Chris. 1995. Possessive Descriptions. Stanford, CA: CSLI Publications.

Barss, Andrew and Howard Lasnik. 1986. A note on anaphora and double objects. Linguistic Inquiry 17: 347-54.

Beaver, David. 1992. The kinematics of presupposition. In P. Dekker and M. Stockhof, eds., Proceedings of the Eighth Amsterdam Colloquium, pages 17-36. Amsterdam: University of Amsterdam Institute for Language, Logic, and Information.

Beavers, John. 2006. Argument/Oblique Alternations and the Structure of Lexical Meaning. Ph.D. thesis, Stanford University.

Beavers, John. 2008. Scalar complexity and the structure of events. In J. Dölling, T. HeydeZybatow, and M. Schäfer, eds., Event Structures in Linguistic Form and Interpretation, pages 245-65. Berlin: Mouton de Gruyter.

Beavers, John. 2010. The structure of lexical meaning: Why semantics really matters. Language 86: 821-64.

Beavers, John. 2011a. An aspectual analysis of ditransitive verbs of caused possession in English. Journal of Semantics 28:1-54.

Beavers, John. 2011b. On affectedness. Natural Language and Linguistic Theory 29:335-70.

Beavers, John. 2012. Lexical aspect and multiple incremental themes. In V. Demonte and L. McNalley, eds., Telicity, Change, and State: A Cross-Categorial View of Event Structure, pages 23-59. Oxford: Oxford University Press.

Beavers, John. 2013. Aspectual classes and scales of change. Linguistics 54: 681-706.

Beavers, John, Michael Everdell, Kyle Jerro, Henri Kauhanen, Andrew Koontz-Garboden, Elise LeBovidge, and Stephen Nichols. 2017. Two types of states: A cross-linguistic study of change-of-state verb roots. In Proceedings of the 91st Annual Meeting of the Linguistic Society of America, pages 38:1-15. Washington, DC: The Linguistics Society of America.

Beavers, John, Michael Everdell, Kyle Jerro, Henri Kauhanen, Andrew Koontz-Garboden, Elise LeBovidge, and Stephen Nichols. 2018. States and changes-of-state: A crosslinguistic study of the roots of verbal meaning. Unpublished ms., The University of Texas at Austin, University of Essex, University of Kontstanz, University of Manchester, University of Washington.

Beavers, John and Andrew Koontz-Garboden. 2012. Manner and result in the roots of verbal meaning. Linguistic Inquiry 43:331-69.

Beavers, John and Andrew Koontz-Garboden. 2013a. Complications in diagnosing lexical meaning: A rejoinder to Horvath and Siloni (2013). Lingua 134:210-8.

Beavers, John and Andrew Koontz-Garboden. 2013b. In defense of the reflexivization analysis of anticausativization. Lingua 131:199-216.

Beavers, John and Andrew Koontz-Garboden. 2017a. Result verbs, scalar change, and the typology of motion verbs. Language 94: 842-76.

Beavers, John and Andrew Koontz-Garboden. 2017b. The semantic contribution of idiosyncratic roots to ditransitive verbs. In Proceedings of the 34th West Coast Conference on Formal Linguistics, pages 70-80. Somerville, MA: Cascadilla Proceedings Project.

Beavers, John and Juwon Lee. In press. Non-culmination in Korean caused change-of-state predicates. Linguistics.

Beavers, John and Chiyo Nishida. 2010. The Spanish dative alternation revisited. In Romance Linguistics 2009: Selected Papers from the 39th Linguistic Symposium of Romance Languages, pages 217-30. Amsterdam: John Benjamins.

Beavers, John, Elias Ponvert, and Stephen Wechsler. 2009. Possession of a controlled substantive: have and other verbs of possession. In T. Friedman and S. Ito, eds., Proceedings of SALT XVIII, pages 108-25. Ithaca: Cornell University. http://hdl.handle.net/1813/13029 
Beavers, John and Cala Zubair. 2013. Anticausatives in Sinhala: Involitivity and causer suppression. Natural Language and Linguistic Theory 31:1-46.

Beck, Sigrid. 2006. Focus on again. Linguistics and Philosophy 29: 277-314.

Beck, Sigrid and Remus Gergel. 2015. The diachronic semantics of again. Natural Language Semantics 23:157-203.

Beck, Sigrid and Kyle Johnson. 2004. Double objects again. Linguistic Inquiry 35: 97-124.

Beck, Sigrid and William Snyder. 2001. The resultative parameter and restitutive again. In C. Fery and W. Sternefeld, eds., Audiatur Vox Sapientiae: A Festschrift for Arnim on Stechow, pages 48-69. Berlin: Akademie Verlag.

Bittner, Maria. 1998. Concealed causatives. Natural Language Semantics 7:1-78.

Bobaljik, Jonathan. 2008. Where's phi? agreement as a post-syntactic operation. In D. Harbour, D. Adger, and S. Bejár, eds., Phi-Theory: Phi Features Across Interfaces and Modules, pages 295-328. Oxford: Oxford University Press.

Bobaljik, Jonathan. 2012. Universals in Comparative Morphology: Suppletion, Superlatives, and the Structure of Words. Cambridge, MA: MIT Press.

Borer, Hagit. 2005. Structuring Sense, Vol. I and II. Oxford: Oxford University Press.

Borer, Hagit. 2013. Structuring Sense, Vol. III. Oxford: Oxford University Press.

Bresnan, Joan and Tatiana Nikitina. 2009. The gradience of the dative alternation. In L. Uyechi and L. H. Wee, eds., Reality Exploration and Discovery: Pattern Interaction in Language and Life, pages 161-84. Stanford, CA: CSLI Publications.

Bruening, Benjamin. 2010a. Ditransitive asymmetries and a theory of idiom formation. Linguistic Inquiry 41: 519-62.

Bruening, Benjamin. 2010b. Double object constructions disguised as prepositional datives. Linguistic Inquiry 41: 287-305.

Chierchia, Gennaro. 2004a. A semantics for unaccusatives and its syntactic consequences. In A. Alexiadou, E. Anagnostopoulou, and M. Everaert, eds., The Unaccusativity Puzzle, pages 22-59. Oxford: Oxford University Press.

Chierchia, Gennaro. 2004b. A semantics for unaccusatives and its syntactic consequences. In A. Alexiadou, E. Anagnostopoulou, and M. Everaert, eds., The Unaccusativity Puzzle, pages 22-59. Oxford: Oxford University Press. Previously circulated as unpublished 1989 Cornell University manuscript.

Comrie, Bernard, Matthew S. Dryer, David Gil, and Martin Haspelmath. 2013. Introduction. In M. S. Dryer and M. Haspelmath, eds., The World Atlas of Language Structures Online, pages 1-9. Leipzig: Max Planck Institute for Evolutionary Anthropology.

Coon, Jessica. 2019. Building verbs in Chuj: Consequences for the nature of roots. Journal of Linguistics 55: 35-81.

Copley, Bridget and Heidi Harley. 2014. Eliminating causative entailments with the forcetheoretic framework: The case of Tono O'odham frustrative cem. In B. Copley and F. Martin, eds., Causation in Grammatical Structures, pages 120-51. Oxford: Oxford University Press.

Copley, Bridget and Heidi Harley. 2015. A force-theoretic framework for event structure. Linguisics and Philosophy 38:103-58.

Copley, Bridget and Phillip Wolff. 2014. Theories of causation should inform linguistic theory and vice versa. In B. Copley and F. Martin, eds., Causation in Grammatical Structures, pages 11-57. Oxford: Oxford University Press.

Cresswell, Max. 1977. The semantics of degree. In B. Partee, ed., Montague Grammar, pages 261-92. New York: Academic Press.

Croft, William. 1990. Possible verbs and the structure of events. In S. L. Tsohatzidis, ed., Meanings and Prototypes, pages 48-73. London: Routledge. 
Croft, William. 1991. Syntactic Categories and Grammatical Relations: The Cognitive Organization of Information. Chicago, IL: University of Chicago Press.

Croft, William. 2012. Verb: Aspect and Causal Structure. Oxford: Oxford University Press.

Cruse, D. A. 1973. Some thoughts on agentivity. Journal of Linguistics 9:11-23.

Davis, Anthony. 2001. Linking by Types in the Hierarchical Lexicon. Stanford, CA: CSLI Publications.

Davis, Anthony and Jean-Pierre Koenig. 2000. Linking as constraints on word classes in a hierarchical lexicon. Language 76: 56-91.

de Swart, Henriëtte. 1998. Aspect shift and coercion. Natural Language and Linguistic Theory 16: 347-85.

Demirdache, Hamida and Fabienne Martin. 2015. Agent control over non culminating events. In E. B. López, J. L. C. Honrubia, and S. R. Rosique, eds., Verbal Classes and Aspect, pages 185-217. Amsterdam: John Benjamins.

Deo, Ashwini, Itamar Francez, and Andrew Koontz-Garboden. 2011. The morphosemantics of $-e d$. Paper presented at Dimensions of grammar in honor of Paul Kiparsky. Konstanz, August 3, 2011.

Deo, Ashwini, Itamar Francez, and Andrew Koontz-Garboden. 2013. From change to value difference in degree achievements. In Proceedings of Semantics and Linguistic Theory 23, pages 97-115. Ithaca, NY: CLC Publications.

Dixon, R. M. W. 1982. Where have all the adjectives gone?: And other essays in semantics and syntax. The Hague: Mouton.

Dixon, R. M. W. 1994. Ergativity. Cambridge: Cambridge University Press.

Doron, Edit. 2003. Agency and voice: The semantics of Semitic templates. Natural Language Semantics 11:1-67.

Doron, Edit. 2009. The interaction of adjectival passive and voice. Handout of talk given at Stanford University, February 17, 2009.

Dowe, Phil. 2000. Physical Causation. Cambridge: Cambridge University Press.

Dowty, David. 1979. Word Meaning and Montague Grammar. Dordrecht: D. Reidel Publishing.

Dowty, David. 1989. On the semantic content of the notion 'thematic role'. In G. Chierchia, B. H. Partee, and R. Turner, eds., Properties, Types, and Meaning, vol. II: Semantic Issues, pages 69-130. Dordrecht: Kluwer.

Dowty, David. 1991. Thematic proto-roles and argument selection. Language 67: 547-619.

Dubinsky, Stanley and Silvester Ron Simango. 1996. Passive and stative in Chichewa: Evidence for modular distinctions in grammar. Language 72(4): 749-81.

Dunbar, Ewan and Alexis Wellwood. 2016. Addressing the "two interface" problem: Comparatives and superlatives. Glossa 1(1):1-29.

Eells, Ellery. 1991. Probabilistic Causality. Cambridge: Cambridge University Press.

Embick, David. 2004. On the structure of resultative participles in English. Linguistic Inquiry 35(3): 355-92.

Embick, David. 2009. Roots, states, and stative passives. Talk given at the 2009 Roots Workshop, Stuttgart.

Fábregas, Antonio. 2007. The Exhaustive Lexicalisation Principle. Nordlyd: TromsøWorking Papers in Linguistics 34(2): 165-99, University of Tromsø, Tromsø, Norway. http://www.ub.uit.no/munin/nordlyd/

Fair, David. 1979. Causation and the flow of energy. Erkenntnis 14: 219-50.

Filip, Hana. 1999. Aspect, Eventuality Types, and Nominal Reference. New York: Garland.

Filip, Hana. 2004. The telicity parameter revisted. In Proceedings of SALT XIV. Ithaca: Cornell University. 
Filip, Hana. 2008. Events and maximalization: The case of telicity and perfectivity. In S. Rothstein, ed., Theoretical and Crosslinguistic Approaches to the Semantics of Aspect, pages 217-56. Amsterdam: John Benjamins.

Fillmore, Charles. 1970. The grammar of hitting and breaking. In R. Jacobs and P. Rosenbaum, eds., Readings in English Transformational Grammar, pages 120-33. Waltham, MA: Ginn.

Fillmore, Charles J. 1968. The case for case. In E. Bach and R. T. Harms, eds., Universals in Linguistic Theory, pages 1-90. New York: Holt.

Fodor, Jerry. 1970. Three reasons for not deriving 'kill' from 'cause to die'. Linguistic Inquiry 1: 429-38.

Folli, Raffaella and Heidi Harley. 2004. Consuming results: Flavours of little- $v$. In P. Kempchimsky and R. Slabakova, eds., Aspectual Inquiries, pages 95-120. Dordrecht: Kluwer.

Folli, Raffaella and Gillian Ramchand. 2002. Event structure composition: The case of goal of motion and resultative constructions in Italian and Scottish Gaelic. In H. J. Verkuyl, ed., Proceedings of Perspectives on Aspect Conference. Utrecht: OTS.

Francez, Itamar and Andrew Koontz-Garboden. 2017. Semantics and Morphosyntactic Variation: Qualities and the Grammar of Property Concepts. Oxford: Oxford University Press.

Garey, Howard B. 1957. Verbal aspects in French. Language 33: 91-110.

Gawron, Mark. 2009. The lexical semantics of extent verbs. San Diego State University, January 31, 2009.

Givón, Talmy. 1984. Syntax: A Functional-Typological Introduction. Amsterdam: John Benjamins.

Goldberg, Adele E. 1995. Constructions: A Construction Grammar Approach to Argument Structure. Chicago, IL: University of Chicago Press.

Goldberg, Adele E. 2010. Verbs, constructions, and semantic frames. In E. Doron, M. Rappaport Hovav, and I. Sichel, eds., Syntax, Lexical Semantics, and Event Structure, pages 39-58. Oxford: Oxford University Press.

Goldberg, Adele E. and Ray Jackendoff. 2004. The English resultative as a family of constructions. Language 80: 532-69.

Goodman, Nelson. 1954. Fact, Fiction, and Forecast. Cambridge, MA: Harvard University Press.

Green, Georgia. 1974. Semantic and Syntactic Regularity. Bloomington: Indiana University Press.

Grimshaw, Jane. 2005. Words and Structure. Stanford, CA: CSLI Publications.

Gropen, Jess, Steven Pinker, Michelle Hollander, Richard Goldberg, and Ronald Wilson. 1989. The learnability and acquisition of the dative alternation in English. Language 65: 203-57.

Gruber, Jeffery. 1965. Studies in Lexical Relations. Ph.D. thesis, MIT. Reprinted as part of Lexical Structures in Syntax and Semantics, North-Holland, Amsterdam, 1976.

Guerssel, Mohamed, Kenneth Hale, Mary Laughren, Levin Beth, and Josie White Eagle. 1985. A cross-linguistic study of transitivity alternations. In Proceedings of the 21st Annual Meeting of the Chicago Linguistic Society, vol. 2: Parasession on Causatives and Agentivity, pages 48-63. Chicago, IL: Chicago Linguistic Society.

Hale, Kenneth L. 1986. Notes on world view and semantic categories: Some Warlpiri examples. In P. Muyskens and H. van Riemsdijk, eds., Features and Projections, pages 233-54. Dordrecht: Foris. 
Hale, Kenneth L. and Samuel Jay Keyser. 1987. A view from the middle. Lexicon Project Working Papers 10, Center for Cognitive Science, MIT, Cambridge, MA.

Hale, Kenneth L. and Samuel Jay Keyser. 1993. On argument structure and the lexical expression of syntactic relations. In K. L. Hale and S. J. Keyser, eds., The View from Building 20: Essays in Linguistics in Honor of Sylvain Bromberger. Cambridge, MA: MIT Press.

Hale, Kenneth L. and Samuel Jay Keyser. 1997. The limits of argument structure. In A. Mendikoetxea and M. Uribe-Etxebarria, eds., Theoretical Issues at the MorphologySyntax Interface, pages 203-30. Bilbao: Universidad de País Vasco, Euskal Herriko Univertsitatea.

Hale, Kenneth L. and Samuel Jay Keyser. 1998. The basic elements of argument structure. In Papers from the UPenn/MIT Roundtable on Argument Structure and Aspect, vol. 32, pages 73-118. MITWPL.

Hale, Kenneth L. and Samuel Jay Keyser. 2002. Prolegomenon to a Theory of Argument Structure. Cambridge, MA: MIT Press.

Halle, Morris and Alec Marantz. 1993. Distributed morphology and the pieces of inflection. In K. Hale and S. J. Keyser, eds., The view from Building 20, pages 111-76. Cambridge, MA: MIT Press.

Harley, Heidi. 2003. Possession and the double object construction. In P. Pica and J. Rooryck, eds., Linguistic Variation Yearbook 2, pages 31-70. Amsterdam: John Benjamins.

Harley, Heidi. 2004. Wanting, having, and getting: A note on Fodor and Lepore 1998. Linguistic Inquiry 35: 255-67.

Harley, Heidi. 2005. How do verbs get their names? Denominal verbs, manner incorporation, and the ontology of verb roots in English. In N. Erteschik-Shir and T. Rapoport, eds., The Syntax of Aspect, pages 42-64. Oxford: Oxford University Press.

Harley, Heidi. 2012. Lexical decomposition in modern syntactic theory. In W. Hinzen, E. Machery, and M. Werning, eds., The Oxford Handbook of Compositionality, pages 328-50. Oxford: Oxford University Press.

Harley, Heidi and Hyun Kyoung Jung. 2015. In support of the $\mathrm{P}_{H A V E}$ analysis of the double object construction. Linguistics Inquiry 46: 703-30.

Harley, Heidi and Shigeru Miyagawa. 2017. Syntax of ditransitives. In Oxford Research Encyclopedia of Linguistics, pages 1-25. Oxford: Oxford University Press.

Haspelmath, Martin. 1993. More on the typology of inchoative/causative verb alternations. In B. Comrie and M. Polinsky, eds., Causatives and Transitivity, pages 87-120. Amsterdam: John Benjamins.

Hay, Jennifer, Christopher Kennedy, and Beth Levin. 1999. Scalar structure underlies telicity in degree achievements. In The Proceedings of SALT IX, pages 127-44.

Hellan, Lars. 1981. Towards an Integrated Analysis of Comparatives. Tübingen: Narr.

Henderson, Robert. 2019. The roots of measurement. Glossa: A Journal of General Linguistics 4(1): 1-32.

Higginbotham, James. 1985. On semantics. Linguistic Inquiry 16: 547-93.

Holisky, Dee Ann. 1987. The case of the intransitive subject in Tsova-Tush (Batsbi). Lingua 71:103-32.

Hopper, Paul J. and Sandra A. Thompson. 1980. Transitivity in grammar and discourse. Language 56: 251-99.

Horvath, Julia and Tal Siloni. 2011. Anticaustives: Against reflexivization. Lingua 121: 2176-86. 
Horvath, Julia and Tal Siloni. 2013. Anticausatives have no cause(r): A rejoinder to Beavers and Koontz-Garboden (in this issue). Lingua 131: 217-30.

Hume, David. 2007 [1784]. An Enquiry Concerning Human Understanding. Cambridge: Cambridge University Press.

Husband, E. Matthew. 2011. Rescuing manner/result complementarity from certain death. In Proceedings from the Annual Meeting of the Chicago Linguistic Society, vol. 47.1, pages 111-24(14). Chicago: Chicago Linguistic Society.

Ikegami, Yoshihiko. 1985. 'Activity'-'accomplishment'-'achievement'- a language that can't say 'I burned it but it didn't burn' and one that can. In A. Makkai and A. K. Melby, eds., Linguistics and Philosophy: Festshrift for Rulon S. Wells, pages 265-304. Amsterdam: John Benjamins.

Inagaki, Shunji. 2001. Motion verbs with locational/directional PPs in English and Japanese. McGill Working Papers in Linguistics 15: 37-79.

Iwata, Seizi. 2005a. Locative alternation and two levels of verb meaning. Cognitive Linguistics 16:355-407.

Iwata, Seizi. 2005b. The role of verb meaning in locative alternations. In M. Fried and H. C. Boas, eds., Grammatical Constructions: Back to the Roots, pages 101-18. Amsterdam: John Benjamins.

Iwata, Seizi. 2008. A door that swings noiselessly open may creak shut: Internal motion and concurrent changes of state. Linguistics 46:1049-1108.

Jackendoff, Ray. 1972. Semantic Interpretation in Generative Grammar. Cambridge, MA: MIT Press.

Jackendoff, Ray. 1990a. On Larson's treatment of the double object construction. Linguistic Inquiry 21: 427-56.

Jackendoff, Ray. 1990b. Semantic Structures. Cambridge, MA: MIT Press.

Jackendoff, Ray. 1996. The proper treatment of measuring out, telicity, and perhaps even quantification in English. Natural Language and Linguistic Theory 14: 305-54.

Jäger, Gerhard and Reinhard Blutner. 2003. Competition and interpretation. In E. Lang, C. Maienborn, and C. Fabricius-Hansen, eds., Modifying Adjuncts, pages 393-416. Berlin: Mouton de Gruyter.

Jerro, Kyle. 2016. The Syntax and Semantics of Applicative Morphology in Bantu. Ph.D. thesis, The University of Texas at Austin.

Jerro, Kyle. 2017a. The causative-instrumental syncretism. Journal of Linguistics 53: 751-88.

Jerro, Kyle. 2017b. Change of state in Kinyarwanda: A study in root meaning. In Proceedings of the Linguistic Society of America, vol. 2, pages 25:1-15. The Linguistics Society of America.

Jespersen, Otto. 1927. A Modern English Grammar on Historical Principles: Part III Syntax, vol. 2. London: George Allen \& Unwin.

Jimenez, Josep Ausensi. 2018. The semantics of kill, murder, and slay. Talk given at Endpoints, Scales, and Results in the Decomposition of Verbal Predicates, January 30th, https://drive.google.com/file/d/0B07XkvUoD1VZaXNHQ2pjRnczUmw5eHNRO V9wVW5vR3EwdndN/view.

Kardos, Éva. 2012. Toward a Scalar Semantic Analysis of Telicity in Hungarian. Ph.D. thesis, University of Debrecen.

Kardos, Éva. 2016. Telicity marking in Hungarian. Glossa: A Journal of General Linguistics $1: 41,1-37$.

Kearns, Kate. 2000. Semantics. New York, NY: St. Martin's Press. 
Keenan, Edward. 1976. Toward a universal definition of 'subject'. In C. Li, ed., Subject and Topic, pages 303-33. New York: Academic Press.

Kehler, Andrew. 2002. Coherence, Reference, and the Theory of Grammar. Stanford, CA: CSLI Publications.

Kelly, Justin. 2013. The Syntax-Semantics Interface in Distributed Morphology. Ph.D. thesis, Georgetown University, Washington, DC.

Kemmer, Suzanne. 1993. The Middle Voice. Amsterdam: John Benjamins.

Kennedy, Christopher. 1997. Projecting the Adjective: The Syntax and Semantics of Gradability and Comparison. Ph.D. thesis, UC-Santa Cruz, Santa Cruz, CA.

Kennedy, Christopher. 2001. Polar opposition and the ontology of 'degrees'. Linguistics and Philosophy 24(1): 33-70.

Kennedy, Christopher. 2007. Vagueness and grammar: The semantics of relative and absolute gradable adjectives. Linguistics and Philosophy 30:1-45.

Kennedy, Christopher and Beth Levin. 2008. Measure of change: The adjectival core of degree achievements. In L. McNally and C. Kennedy, eds., Adjectives and Adverbs: Syntax, Semantics, and Discourse, pages 156-82. Oxford: Oxford University Press.

Kiparsky, Paul. 1997. Remarks on denominal verbs. In A. Alsina, J. Bresnan, and P. Sells, eds., Argument Structure, pages 473-99. Stanford, CA: CSLI Publications.

Kistler, Max. 2014. Analyzing causation in light of intuitions, causal statements, and science. In B. Copley and F. Martin, eds., Causation in Grammatical Structures, pages 76-99. Oxford: Oxford University Press.

Koenig, Jean-Pierre and Liangcheng Chief. 2008. Scalarity and state-changes in Mandarin (and other languages). In O. Bonami and P. C. Hofherr, eds., Empirical Issues in Syntax and Semantics 7, pages 241-62.

Koenig, Jean-Pierre, Liancheng Chief, Nuttanart Muansuwan, and Poornima Shakti. 2016. A shopping guide to cross-linguistic variation in event realization. Unpublished $\mathrm{ms}$, The University at Buffalo, State University of New York.

Koenig, Jean-Pierre and Anthony R. Davis. 2001. Sublexical modality and the structure of lexical semantic representations. Linguistics and Philosophy 24: 71-124.

Koenig, Jean-Pierre and Anthony R. Davis. 2006. The KeY to lexical semantic representations. Journal of Linguistics 42: 71-108.

Koenig, Jean-Pierre and Nuttannart Muansuwan. 2000. How to end without ever finishing: Thai semi-perfectivity. Journal of Semantics 17:147-82.

Koontz-Garboden, Andrew. 2005. On the typology of state/change of state alternations. Yearbook of Morphology 2005: 83-117.

Koontz-Garboden, Andrew. 2006. The states in changes of state. Proceedings of the 32nd Annual Meeting of the Berkeley Linguistics Society, pages 515-27.

Koontz-Garboden, Andrew. 2007. Aspectual coercion and the typology of change of state predicates. Journal of Linguistics 43(1):115-52.

Koontz-Garboden, Andrew. 2009a. Anticausativization. Natural Language and Linguistic Theory 27: 77-138.

Koontz-Garboden, Andrew. 2009b. Ulwa verb class morphology. International Journal of American Linguistics 75(4): 453-512.

Koontz-Garboden, Andrew. 2010. The lexical semantics of derived statives. Linguistics and Philosophy 33: 285-324.

Koontz-Garboden, Andrew. 2016. Thoughts on diagnosing morphomicity: A case study from Ulwa. In A. Luís and R. Bermúdez-Otero, eds., The Morphome Debate: Diagnosing and Analysing Morphomic Patterns, pages 89-111. Oxford: Oxford University Press. 
Koontz-Garboden, Andrew and John Beavers. 2017. Change of state verbs and the semantics of roots. In Proceedings of the 34th West Coast Conference on Formal Linguistics. Somerville, MA: Cascadilla Proceedings Project.

Koontz-Garboden, Andrew and Beth Levin. 2005. The morphological typology of change of state event encoding. In G. Booij, E. Guevara, A. Ralli, S. Sgroi, and S. Scalise, eds., Online Proceedings of the Fourth Mediterranean Morphology Meeting (MMM4) Catania, 21-23 September 2003, pages 185-94. Università degli Studi di Bologna. http://www.lilec.it/ mmm/wp/wp-content/uploads/2012/09/185-194-KoontzGarboden-Levin-MMM4.pdf.

Kratzer, Angelika. 1996. Severing the external argument from its verb. In J. Rooryck and L. Zaring, eds., Phrase Structure and the Lexicon, pages 109-37. Dordrecht: Kluwer.

Kratzer, Angelika. 2000. Building statives. In Proceedings of the 26th Meeting of the Berkeley Linguistics Society, pages 385-99. Berkeley, CA: Berkeley Linguistics Society.

Kratzer, Angelika. 2005. Building resultatives. In C. Maienborn and Wöllstein-Leisten, eds., Event Arguments: Functions and Applications, pages 177-212. Tübingen: Niemeyer.

Krifka, Manfred. 1989. Nominal reference, temporal constitution, and quantification in event semantics. In R. Bartsch, J. van Benthem, and P. van Emde Boas, eds., Semantics and Contextual Expressions, pages 75-115. Dordrecht: Foris.

Krifka, Manfred. 1992. Thematic relations as links between nominal reference and temporal constitution. In I. Sag and A. Szabolcsi, eds., Lexical Matters, pages 29-54. Stanford, CA: CSLI Publications.

Krifka, Manfred. 1998. The origins of telicity. In S. Rothstein, ed., Events and Grammar, pages 197-235. Dordrecht: Kluwer.

Krifka, Manfred. 2004. Semantic and pragmatic conditions for the dative alternation. Korean Journal of English Language and Linguistics 4:1-32.

Krohn, Laurie. 2008. The manner/result complementarity and verbs of the manner of death. Unpublished undergraduate Grammatical Semantics class essay, The University of Manchester.

Lagnado, David, Michael Waldmann, York Hagmayer, and Steven Sloman. 2007. Beyond covariation: Cues to causal structure. In A. Gopnik and L. Schultz, eds., Causal Learning: Psychology, Philosophy, and Computation, pages 154-72. Oxford: Oxford University Press.

Lakoff, George. 1965. On the Nature of Syntactic Irregularity. Ph.D. thesis, Indiana University, Bloomington, IN. Published 1970 as Irregularity in Syntax. New York: Holt, Rinehart, and Winston.

Langacker, Ronald W. 2005. Construction Grammars: Cognitive, radical, and less so. In F. J. R. de Mendoza Ibáñez and M. S. Peña Cervel, eds., Cognitive Linguistics: Internal Dynamics and Interdisciplinary Interaction, pages 101-59. Berlin: Mouton de Gruyter.

Larson, Richard K. 1988. On the double object construction. Linguistic Inquiry 19: 335-92.

Larson, Richard K. 1990. Double objects revisited: Reply to Jackendoff. Linguistic Inquiry 21: 589-632.

Levin, Beth. 1993. English Verb Classes and Alternations: A Preliminary Investigation. Chicago, IL: University of Chicago Press.

Levin, Beth. 1999. Objecthood: An event structure perspective. In Proceedings of CLS 35, pages 223-47.

Levin, Beth. 2008. A constraint on verb meanings: Manner/result complementarity. Handout of talk presented at Cognitive Science Department Colloqium Series, Brown University, Providence, RI, March 17, 2008.

Levin, Beth and Tova Rapoport. 1988. Lexical subordination. In CLS 24, pages 275-89. 
Levin, Beth and Malka Rappaport Hovav. 1991. Wiping the slate clean: A lexical semantic exploration. Cognition 41:123-51.

Levin, Beth and Malka Rappaport Hovav. 1995. Unaccusativity: At the Syntax-Lexical Semantics Interface. Cambridge, MA: MIT Press.

Levin, Beth and Malka Rappaport Hovav. 2005. Argument Realization. Cambridge: Cambridge University Press.

Levin, Beth and Malka Rappaport Hovav. 2008. Lexicalized manner and result are in complementary distribution. Handout of talk given at the 24th meeting of the Israeli Association for Theoretical Linguistics, October 26-7, 2008.

Levin, Beth and Malka Rappaport Hovav. 2013. Lexicalized meaning and manner/result complementarity. In B. Arsenijević, B. Gehrke, and R. Marín, eds., Subatomic Semantics of Event Predicates, pages 49-70. Dordrecht: Springer.

Levinson, Lisa. 2007. The Roots of Verbs. Ph.D. thesis, NYU, New York.

Lewis, David. 1973. Causation. The Journal of Philosophy 70: 556-67.

Lewis, David. 2000. Causation as influence. The Journal of Philosophy 97: 409-21.

Link, Godehard. 2002. The logical analysis of plurals and mass terms: A lattice-theoretical approach. In P. Portner and B. Partee, eds., Formal Semantics: The Essential Readings, pages 127-46. Oxford: Blackwell. Originally published 1983 in R. Bäuerle, Chr. Schwarze, and A. von Stechow, eds., Meaning, Use, and Interpretation of Language, pages 302-23. Berlin: de Gruyter.

Lundquist, Björn, Martin Corley, Mai Tungseth, Antonella Sorace, and Gillian Ramchand. 2016. Anticausatives are semantically reflexive in Norwegian, but not in English. Glossa $1(1): 47.1-30$.

Marantz, Alec. 1996. Cat as a phrasal idiom: Consequences of late insertion in Distributed Morphology. Ms., MIT.

Marantz, Alec. 1997. No escape from syntax: Don't try morphological analysis in the privacy of your own lexicon. In Proceedings of the 21st Annual Penn Linguistics Colloquium, vol. 4.2 of University of Pennsylvania Working Papers in Linguistics, pages 201-25.

Marantz, Alec. 2007. Restitutive re- and the first phase syntax/semantics of the VP. Talk given at the University of Maryland.

Marantz, Alec. 2009. Roots, re-, and affected agents: can roots pull the agent under little v. Talk given at Roots, Universitat Stuttgart, June 2009.

Martin, Fabienne and Florian Schäfer. 2012. The modality of offer and other defeasible causative verbs. In Proceedings of the 30th West Coast Conference on Formal Linguistics, pages $248-58$.

Martin, Fabienne and Florian Schäfer. 2017. Sublexical modality in defeasible causative verbs. In A. Arregui, M. L. Rivero, and A. Salanova, eds., Modality Across Syntactic Categories, pages 87-108. Oxford: Oxford University Press.

Mateu, Jaume and Víctor Acedo-Matellán. 2012. The manner/result complementarity revisited: A syntactic approach. In M. C. Cuervo and Y. Roberge, eds., The End of Argument Structure, vol. 28 of Syntax and Semantics, pages 209-28. London: Emerald Group Publishing.

Matthewson, Lisa, Heidi Quinn, and Lynsey Talagi. 2015. Inchoativity meets the perfect time span: The Niuean perfect. Lingua 168: 1-36.

McCawley, James. 1968. Lexical insertion in a transformational grammar without deep structure. In Proceedings of the Fourth Regional Meeting of the Chicago Linguistic Society, pages 71-80. Chicago, IL: Chicago Linguistic Society. 
McCawley, James D. 1971. Prelexical syntax. In Report of the 22nd Annual Roundtable Meeting on Linguistics and Language Studies, pages 19-33. Washington, DC: Georgetown University Press.

McCawley, James D. 1974. On identifying the remains of deceased clauses. Language Research 9: 73-85.

Megerdoomian, Karine. 2002. Beyond Words and Phrases: A Unified Theory of Predicate Composition. Ph.D. thesis, University of Southern California, Los Angeles, CA.

Müller, Ana and Luciana Sanchez-Mendes. 2008. Pluractionality in Karitiana. In A. Grønn, ed., Proceedings of Sinn und Bedeutung 12, pages 442-54. ILOS: University of Oslo.

Myler, Neil. 2014. Building and Interpreting Possessive Sentences. Ph.D. thesis, NYU, New York, NY.

Nedjalkov, Vladimir P. and Sergej Je. Jaxontov. 1988. The typology of resultative constructions. In V. P. Nedjalkov, ed., Typology of Resultative Constructions, pages 3-62. Amsterdam: John Benjamins.

Nichols, Johanna, David A. Peterson, and Jonathan Barnes. 2004. Transitivizing and detransitivizing languages. Linguistic Typology 8:149-211.

Oehrle, Richard T. 1976. The Grammatical Status of the English Dative Alternation. Ph.D. thesis, MIT.

Parsons, Terence. 1990. Events in the Semantics of English: A Study in Subatomic Semantics. Cambridge, MA: MIT Press.

Partee, Barbara H. 1999. Weak NP's in HAVE sentences. In J. Gerbrandy, M. Marx, M. de Rijke, and Y. Venema, eds., JFAK, a Liber Amicorum for Johan van Benthen on the occasion of his 50th Birthday, pages 39-57. Amsterdam: University of Amsterdam.

Pedersen, Walter A. 2014. A scalar analysis of again-ambiguities. Journal of Semantics 32: 373-424.

Perlmutter, David M. and Paul Postal. 1984. The 1-Advancement Exclusiveness Law. In D. M. Perlmutter and C. Rosen, eds., Studies in Relational Grammar 2. Chicago, IL: University of Chicago Press.

Pesetsky, David. 1995. Zero Syntax: Experiencer and Cascades. Cambridge, MA: MIT Press.

Peterson, David A. 2007. Applicative Constructions. Oxford: Oxford University Press.

Pinkal, Manfred. 1989. On the logical structure of comparatives. In R. Studer, ed., Lecture Notes in Artificial Intelligence, pages 146-67. Berlin: SpringerVerlag.

Pinker, Steven. 1989. Learnability and Cognition: The Acquisition of Argument Structure. Cambridge, MA: MIT Press.

Primus, Beatrice. 1999. Case and Thematic Roles: Ergative, Accusative and Active. Tübingen: Niemeyer.

Pross, Tillmann. 2019. What about lexical semantics if syntax is the only generative component of grammar. Natural Language and Linguistic Theory 37: 215-61.

Pylkkänen, Liina. 2008. Introducing Arguments. Cambridge, MA: MIT Press.

Ramchand, Gillian Catriona. 2008. Verb Meaning and the Lexicon: A First-phase Syntax. Cambridge: Cambridge University Press.

Rappaport, Malka and Beth Levin. 1988. What to do with $\theta$-roles. In W. Wilkins, ed., Thematic Relations, pages 7-36. San Diego, CA: Academic Press.

Rappaport Hovav, Malka. 2008. Lexicalized meaning and the internal structure of events. In S. Rothstein, ed., Theoretical and Crosslinguistic Approaches to the Semantics of Aspect, pages 13-42. Amsterdam: John Benjamins.

Rappaport Hovav, Malka. 2010. Building scalar changes. The Hebrew University of Jerusalem. 
Rappaport Hovav, Malka. 2015. Towards an understanding of the notions of 'manner' and 'result' and their role in the construction of verb meaning. Talk given at Roots IV, New York University, June 28-9.

Rappaport Hovav, Malka and Beth Levin. 1998. Building verb meanings. In M. Butt and W. Geuder, eds., The Projection of Arguments: Lexical and Compositional Factors, pages 97-133. Stanford, CA: CSLI Publications.

Rappaport Hovav, Malka and Beth Levin. 2001. An event structure account of English resultatives. Language 77: 766-97.

Rappaport Hovav, Malka and Beth Levin. 2008. The English dative alternation: A case for verb sensitivity. Journal of Linguistics 44:129-67.

Rappaport Hovav, Malka and Beth Levin. 2010. Reflections on manner/result complementarity. In E. Doron, M. Rappaport Hovav, and I. Sichel, eds., Syntax, Lexical Semantics, and Event Structure, pages 21-38. Oxford: Oxford University Press.

Reichenbach, Hans. 1956. The Direction of Time. Los Angeles: University of California Press.

Reinhart, Tanya. 1996. Syntactic effects of lexical operations: Reflexives and unaccusatives. In OTS Working Papers. Utrecht: Universiteit Utrecht. Downloaded from author's website: http://www.let.uu.nl/ tanya.reinhart/personal/

Reinhart, Tanya. 2002. The theta system - an overview. Theoretical Linguistics 28: 229-90.

Richards, Norvin. 2001. An idiomatic argument for lexical decomposition. Linguistic Inquiry 32(1): 183-92.

Rissman, Lilia. 2016. Cinderella broke and broke: Object deletion and manner-result complementarity. In Proceedings of the 51st Chicago Linguistics Society, pages 425-39. Chicago Linguistics Society.

Ross, John Robert. 1972. Act. In D. Davidson and G. Harman, eds., Semantics of Natural Language, pages 70-126. Dordrecht: Reidel.

Ross, John Robert. 1976. To have have and to not have have. In M. A. Jazayery, E. C. Polomé, and W. Winter, eds., Linguistic and Literary Studies in Honor of Archibald A. Hill, pages 263-70. The Hague: Mouton.

Rothstein, Susan. 2004. Structuring Events. Oxford: Blackwell.

Salmon, Wesley. 1984. Scientific Explanation and the Causal Structure of the World. Princeton: Princeton University Press.

Schäfer, Florian. 2009. The causative alternation. Language and Linguistics Compass 3(2): Richards, Norvin. 2001. An idiomatic argument for lexical decomposition. Linguistic Inquiry pages 183-192. 641-81.

Schein, Barry. 1993. Plurals and Events. Cambridge, MA: MIT Press.

Schwarzschild, Roger and Karina Wilkinson. 2002. Quantifiers in comparatives: A semantics of degree based on intervals. Natural Language Semantics 10:1-41.

Shibatani, Masayoshi. 1976. The grammar of causative constructions: A conspectus. In M. Shibatani, ed., The Grammar of Causative Constructions, pages 1-39. New York: Academic Press.

Shibatani, Masayoshi and Prashant Pardeshi. 2001. The causative continuum. In M. Shibatani, ed., The Grammar of Causation and Interpersonal Manipulation, pages 85-126. Amsterdam: John Benjamins.

Simpson, Jane. 1983. Resultatives. In L. Levin, M. Rappaport, and A. Zaenen, eds., Papers in Lexical-Functional Grammar, pages 143-57. Bloomington: Indiana University Linguistics Club.

Singh, Mona. 1998. On the semantics of the perfective aspect. Natural Language Semantics 6:171-99. 
Smith, Marcus. 2006. Canalization of causative derivations. Paper presented at 2006 meeting of LSA, Albuquerque, New Mexico.

Snyder, Kieran Margaret. 2003. The Relationship between Form and Function in Ditransitive Constructions. Ph.D. thesis, University of Pennsylvania, Philadelphia.

Snyder, William. 2001. On the nature of syntactic variation: Evidence from complex predicates and complex word-formation. Language 77: 324-42.

Spathas, Giorgos. 2017. Results in the decomposition of verbal predicates: Evidence from additive operators. Talk given at The Building Blocks and Mortar of Meaning II, University of Tübingen, November 3-4.

Stalnaker, Robert. 1968. A theory of conditionals. In Studies in Logical Theory, pages 98-112. Oxford: Blackwell.

Stringer, David. 2001. The syntax of paths and boundaries. In Proceedings of CLS 37, pages 139-53. Chicago, IL.

Stringer, David. 2005. Children's structuring of motion events: Syntactic universals and lexical variation. In 6th Tokyo Conference on Psycholinguistics, pages 319-43. Tokyo: Hituzi Syobo.

Stringer, David. 2006. Typological tendencies and universal grammar in the acquisition of adpositions. In P. Saint-Dizier, ed., Syntax and Semantics of Prepositions, pages 57-68. Dordrecht: Springer.

Suppes, Patrick. 1970. A Probabilistic Theory of Causality. Amsterdam: North-Holland.

Talmy, Leonard. 1976. Semantic causative types. In M. Shibatani, ed., The Grammar of Causative Constructions, pages 43-116. New York: Academic Press.

Talmy, Leonard. 1988. Force dynamics in language and cognition. Cognitive Science 12: 49-100.

Talmy, Leonard. 2000. Toward a Cognitive Semantics. Cambridge, MA: MIT Press.

Tatevosov, Sergie. 2008. Subevental structure and non-culmination. In O. Bonami and P. C. Hofherr, eds., Empirical Issues in Syntax and Semantics 7, pages 393-422.

Tatevosov, Sergei. 2012. Telicity, measures, and endpoints. In Proceedings of Sinn und Bedeutung 18, pages 585-98. MITWPL.

Tenny, Carol. 1987. Grammaticalizing Aspect and Affectedness. Ph.D. thesis, MIT.

Tenny, Carol. 1992. The aspectual interface hypothesis. In I. A. Sag and A. Szabolcsi, eds., Lexical Matters, pages 490-508. Stanford, CA: CSLI Publications.

Tenny, Carol. 1994. Aspectual Roles and the Syntax-Semantic Interface. Dordrecht: Kluwer.

Tham, Shiao Wei. 2006. The definiteness effect in English Have sentences. In P. Denis, E. McCready, A. Palmer, and B. Reese, eds., TLS 8, pages 137-49. Somerville, MA: Cascadilla Proceedings Project.

Thompson, Sandra. 1988. A discourse approach to the cross-linguistic category 'adjective'. In J. A. Hawkins, ed., Explaining Language Universals, pages 167-85. Oxford: Basil Blackwell.

Travis, Lisa. 2000. Event structure in syntax. In C. Tenny and J. Pustejovsky, eds., Events as Grammatical Objects: The Converging Perspectives of Lexical Semantics and Syntax, pages 145-85. Stanford, CA: CSLI Publications.

Valle, Daniel, John Beavers, and Andrew Koontz-Garboden. 2017. State and change of state in Kakataibo: The role of root semantics. Paper presented at the annual meeting of the Society for the Study of the Indigenous Languages of the Americas, Austin, Texas, January 5-8.

van Riemsdijk, Henk. 2007. Case in spatial adpositional phrases: The dative-accusative alternation in German. In G. Alboiu, A. A. Avram, L. Avram, and D. Isac, eds., Pitar Mos: A Building With A View. Papers in Honour of Alexandra Cornilescu, pages 265-83. Bucharest: Bucharest University Press. 
van Riemsdijk, Henk and Riny Huijbregts. 2007. Locations and locality. In S. Karimi, V. Samiian, and W. K. Wilkins, eds., Phrasal and Clausal Architecture: Syntactic Derivation and Interpretation: In honor of Joseph E. Emonds, pages 339-64. Amsterdam: John Benjamins.

Van Valin, Robert D., Jr. and Randy J. LaPolla. 1997. Syntax: Structure, Meaning, and Function. Cambridge: Cambridge University Press.

Van Valin, Robert D., Jr. and David P. Wilkins. 1996. The case for "effector": Case roles, agents, and agency revisited. In M. Shibatani and S. A. Thompson, eds., Grammatical Constructions: Their Form and Meaning, pages 289-322. Oxford: Oxford University Press.

Van Valin, Robert D. and Randy J. LaPolla. 1997. Syntax: Structure, Meaning, and Function. Cambridge: Cambridge University Press.

Van Valin, Robert D. and David P. Wilkins. 1996. The case for "effector": Case roles, agents and agency revisited. In M. Shibatani and S. A. Thompson, eds., Grammatical Constructions: Their Form and Meaning, pages 289-322. Oxford: Oxford University Press.

Van Voorst, Jan. 1995. The semantic structure of causative constructions. Studies in Language 19: 489-523.

Vendler, Zeno. 1957. Verbs and times. Philosophical Review 56:143-60.

Verkuyl, Henk J. 1972. On the Compositional Nature of the Aspects, vol. 15 of Foundation of Language Supplementary Series. Dordrecht: Reidel.

Verkuyl, Henk J. 1993. A Theory of Aspectuality. Cambridge: Cambridge University Press.

Verspoor, Cornelia Maria. 1997. Contextually-Dependent Lexical Semantics. Ph.D. thesis, University of Edinburgh, Edinburgh.

Vikner, Carl and Per Anker Jensen. 2002. A semantic analysis of the English genitive. interaction of lexical and formal semantics. Studia Linguistica 56:191-226.

von Stechow, Arnim. 1984. Comparing semantic theories of comparison. Journal of Semantics 3:1-77.

von Stechow, Arnim. 1995. Lexical decomposition in syntax. In U. Egli, P. E. Pause, C. Schwarze, A. von Stechow, and G. Weinold, eds., The Lexicon in the Organization of Language, pages 81-118. Amsterdam and Philedelphia: John Benjamins.

von Stechow, Arnim. 1996. The different readings of wieder 'again': A structural account. Journal of Semantics 13: 87-138.

von Stechow, Arnim. 2003. How are results represented and modified? Remarks on Jäger \& Blutner's anti-decomposition. In E. Lang, C. Fabricius-Hansen, and C. Maienborn, eds., Modifying Adjuncts, pages 417-54. Berlin: Mouton.

Washio, Ryuichi. 1997. Resultatives, compositionality, and language variation. Journal of East Asian Linguistics 6:1-49.

Wasow, Thomas. 2002. Postverbal Behavior. Stanford, CA: CSLI Publications.

Wechsler, Stephen. 1989. Accomplishments and the verbal prefix re-. In Northeastern Linguistics Society (NELS) 19, pages 419-34. Amherst: Graduate Linguistic Student Association.

Wechsler, Stephen. 1995. The Semantic Basis of Argument Structure. Stanford, CA: CSLI Publications.

Wechsler, Stephen. 1997. Resultative predicates and control. In Texas Linguistics Forum 38: The Syntax and Semantics of Predication, pages 307-21. The University of Texas at Austin, Austin, TX.

Wechsler, Stephen. 2005a. Resultatives under the 'event-argument homomorphism' model of telicity. In N. Erteschik-Shir and T. Rapoport, eds., The Syntax of Aspect, pages 255-73. Oxford: Oxford University Press. 
Wechsler, Stephen. 2005b. What is right and wrong about little- $v$. In M. Vulchanova and T. A. Åfarli, eds., Grammar and Beyond-Essays in Honour of Lars Hellan, pages 179-95. Oslo: Novus Press.

Wierzbicka, Anna. 1998. The semantics of English causative constructions in a universaltypological perspective. In M. Tomasello, ed., The New Psychology of Language: Cognitive and Functional Approaches to Language Structure, vol. 1, pages 113-53. Mahwah, NJ: Lawrence Erlbaum Associates.

Williams, Alexander. 2015. Arguments in Syntax and Semantics. Cambridge: Cambridge University Press.

Wolff, Phillip. 2003. Direct causation in the linguistic encoding and individuation of causal events. Cognition 88:1-48.

Wolff, Phillip. 2007. Representing causation. Journal of Experimental Psychology 136: 82-111.

Wolff, Phillip. 2014. Causal pluralism and force dynamics. In B. Copley and F. Martin, eds., Causation in Grammatical Structures, pages 100-119. Oxford: Oxford University Press.

Wolff, Phillip, Aron K. Barbey, and Matthew Hausknecht. 2010. For want of a nail: How absences cause events. Journal of Experimental Psychology: General 139:191-221.

Wolff, Phillip, Bianca Klettke, Tatyana Ventura, and Grace Song. 2005. Expressing causation in English and other languages. In Categorization Inside and Outside the Laboratory, pages 29-48. Washington, DC: American Psychological Association.

Wolff, Phillip and Grace Song. 2003. Models of causation and the semantics of causal verbs. Cognitive Psychology 47:1-57.

Wood, Jim and Alec Marantz. 2015. The interpretation of external arguments. http:// ling.auf.net/lingbuzz/002487

Wunderlich, Dieter. 1997. Cause and the structure of verbs. Linguistic Inquiry 28: 27-68.

Zucchi, Sandro and Michael White. 2001. Twigs, sequences and the temporal constitution of predicates. Linguistics and Philosophy 24: 223-70.

Zwarts, Joost. 2006. Event shape: Paths in the semantics of verbs. Paper presented at the Workshop on the geometric structure of events, Konstanz October 7-8, 2004.

Zwarts, Joost. 2008. Aspects of a typology of direction. In S. Rothstein, ed., Theoretical and Crosslinguistic Approaches to the Semantics of Aspect, pages 79-106. Amsterdam: John Benjamins.

Zwicky, Arnold M. and Jerrold M. Sadock. 1975. Ambiguity tests and how to fail them. In J. P. Kimball, ed., Syntax and Semantics, vol. 4, pages 1-36. New York: Academic Press. 



\section{Index}

A-movement 14

Ackerman, F. and J. Moore 217

ACT 10, 11n4

action

complexity $177-8$

denial $175-7$

actorhood 173-7

adjectival predicates $41-2$

adjectival structures $60-7$

adjectives

comparative 42,43

derived from roots $76-80$

deverbal 94

maximal endpoint 42-3, 47

minimal endpoint $41-2,43 \mathrm{n} 24$

open scale 41-2

positive standard $41-2,44,45-8$

adverbial modifiers $83-9,93,103$

again 16-18, 19, 20, 24, 30, 44, 83-9, 93, 103, 152-3, 154, 195-200, 202, 203-4, 206-8, 222

Agent Control Hypothesis 51

agent role $6,7,11,13$

agentivity 174

Alexiadou, A. et al. 22, 102

alienable possession 113

allomorphy $151,152-5$

allosemy 98-102, 150-1, 152-5

applicatives 155

Arad, M. 26, 32, 220

argument agreement 2-3, 7

Argument-per-Subevent Condition 168

arrival roots $138-40$

aspect and causation 53-5

aspectual motivations for change of state 35-41

attributive modification $64 \mathrm{n} 8$

Baker, M. C. 60 n4, $94 n 28$

Bale, A. C. 94

ballistic motion verbs $117-18,126 n 11,136$, 145-6, 151-2, 154, 178, 187-9, 203-5, 208, 219-20

Bar-el, L. et al. 53

Barss, A. and H. Lasnik 109, 156

basic states $60,74,78,79,92 \mathrm{n} 26,93$
Beaver, D. $16 \mathrm{n} 9$

Beavers, J. 38-40, 45, 53, 107n1, 111, 114, 121, $122,124,127,133 \mathrm{n} 17,136,142,149$, $166-7,171,188,217,218,219$ n1, 224-6

Beavers, J. and A. Koontz-Garboden 42n23, 92, $107 \mathrm{n} 1,138 \mathrm{n} 20,165-6,171,222,230$

Beavers, J. and J. Lee 52

Beavers, J. and C. Zubair 14n8, 92

Beavers, J. et al. 76, 78-80, 90n23, 92, 113

Beck, S. 16n9

Beck, S. and R. Gergel 88

Beck, S. and K. Johnson 103, 151, 195, 203

Beck, S. and W. Snyder 195

BECOME 10-11, 92

become' 26, 35-6, 46-8, 81-4, 99, 122, 125, 128-39, 144, 152, 200-2, 215-16, 221,227

Bifurcation Thesis of Roots 26-7, 33-4, 57, 59-105, 110, 206-9, 213-34

morphological predictions $74-6,80$

Bittner, M. 51

Bobaljik, J. 2, 46n26

Borer, H. 25, 32

break 3, 170

Bresnan, J. and T. Nikitina 148-9

Bruening, B. 154

causation 126, 128, 175-6, 208

and aspect $53-5$

theories of $48-55$

causatives $75,78,92-3,100,174$

CAUSE 10-11, 13, 21, 92, 175

cause' $14-15,17-18,24,26,48-53,126$, 128-34, 137-9, 152, 201, 215-16, 227

caused possession $34,187-9$

ditransitive verbs $107-14,120$

central coincidence 114

change-of-state verbs $3-4,7,8-9,10-12,14-21$, $28,213-14,221,222,228$

aspectual motivations $35-41$

Bifurcation Thesis 57, 59-105, 110

causation 50, 51

deadjectival and non-deadjectival 58-9, 78

durativity $142-8$

entailments of change in $16,19,24,51,58$, $62-80,81,122-4,165-72$ 
change-of-state verbs (cont.)

roots vs. templates 104

scales and comparison 41-8

Chichewa $67 \mathrm{n} 10$

Chierchia, G. 92

circular motion $138 \mathrm{n} 20$

Cognitive Grammar 219n2

co-location $111,114,117,120,151-3$

comparative adjectives 42,43

complementarity 27-8, 29-30, 178n15, 191-5 see also Manner/Result Complementarity

complex root meanings 214-16, 223

complexity of action $177-8$

compositional analysis, of ditransitive verbs $124-30$

constraints on root meanings 224-6

Construction Grammar 12n5, 26-7n12

control possession 113

cooking verbs $178,184-7,197-9,207-8,221-2$

Coon, J. 14

Copley, B. and P. Wolff 48-50

crack class of roots $33,216,221-2$

Cresswell, M. 44, 45

crucify 223

culmination and non-culmination readings $51-3$

dative alternation $107-9,148-50$

deadjectival verbs $43 \mathrm{n} 24$

and non-deadjectival change-of-state verbs $58-9,78$

Demirdache, H. and F. Martin 51

denial of action 175-7

denial of result $166-7$

Deo, A. et al. $16 n 9,67 n 10,72,88$

dependency theories of causation 48-50

derived statives 67

destroy 225

deverbal adjectives 94

deverbal structures $64-5,67-71$

dim 169-70

Distributed Morphology (DM) 12, 81, 102

ditransitive verbs

of caused possession 187-9

compositional analysis 124-30

roots and templates $107-11,155-7$

telicity $122-4$

Dixon, R. M. W. 58

Doron, E. 94

Dowty, D. 3n1, 7-8, 20, 23, 24, 27n12, 31, 32, $33,36,91,93-4,113,126 \mathrm{n} 11,146,177$, 180n17, 213, 217, 220n2, 224-6, 234

drown 230 dry 10

Dubinsky, S. and S. R. Simango 67n10

durativity $40-1,48,54,142-8,177$

Eastern Armenian 76, 77

effector $14 \mathrm{n} 8$

electronic release and arrival roots $139-40$

embeddedness of arguments in event structure 11-12

Embick, D. 26, 33, 60-2, 64n8, 74-5, 77, 96, 100

entailments of change $16,19,24,51,58,62-80$, $81,122-4,165-72$

ergative/absolutive languages 2

event complexity 54,142

event structures $6,8-25,125,213$

lexicalist $10-11,19-21,90-5,110 \mathrm{n} 3$

manner/result complementarity in 192-5

non-lexicalist $81-90,94-5$

event templates $9,12,13,21,22,26$

eventive verbs 160

existential closure of event variable 15

extended letting 175

Figure/Path Relation (FPR) 39, 40-1, 53

Filip, H. 38n16

Fillmore, C. 3-4

flatten 8-9, 10, 11-12, 14-18, 20-1, 30, 33

focus possession 113

Fodor, J. 21

Francez, I. and A. Koontz-Garboden $43 \mathrm{n} 25$

French 52

future having roots $140-1$

Gawron, M. 14n8, 81n17

German, stative participles $82 \mathrm{n} 18$

giving verbs $115-17,120-1$

Goldberg, A. E. 26, 184, 220n2

Goodman, N. 225

gradability of change $144-5,186 \mathrm{n} 21$

Greek 76, 88-9

Green, G. 113

Grimshaw, J. 27, 159

Gropen, J. et al. 187

grue 225-6

guillotine 201-2

Hakha Lai 155

Hale, K. L. 114

Hale, K. L. and S. J. Keyser 60n4, 94n28, 114

handing 25n11, 216, 222, 226, 229-30

Harley, H. 31, 108, 110, 114, 125, 156

Harley, H. and H. K. Jung 108, 110n4

Haspelmath, M. 91 
Hay, J. et al. 38

head movement 13,14

Hebrew 32, 76, 94n29, 220

Hellan, L. 45

Henderson, R. 14

hit 3,11

Holisky, D. A. 177

homomorphic relations 37-41

Hopper, P. J. and S. A. Thompson 23, 32

human agents $67 \mathrm{n} 9$

Hume, D. 49

Husband, E. M. 190-1

inalienable possession 113

inchoative verbs $12,15,63,65,75,78,92-3$, 100,174

indirect object templates $115-17,120$

instrument role 6-7

intentionality in non-culmination 52

Iwata, S. $27 \mathrm{n} 12$

Jackendoff 21, 110n3, 119, 123

Jäger, G. and R. Blutner 93n27

Jerro, K. 155

Jespersen, O. 180n 17

jog $9,10,12$

Kakataibo 78

Karachay-Balkar 51

Kardos, É. 38n16, 45

Karitiana 143

Kelly, J. 23, 32

Kennedy, C. 45

Kennedy, C. and B. Levin 42, 43

killing verbs $1-3,5,6,21,50,178,179-84$, 196-7, 206-7, 223

Kinyarwanda 78, 155

Kiparsky, P. 25n11

Koenig, J.-P. and A. R. Davis 176n14

Koenig, J.-P. and N. Muansuwan 53

Koontz-Garboden, A. 14n8, 23, 67n10, 71, 78, $8 \ln 17,92$

Korean 51-2

Kratzer, A. 20, 64n7, 82n18, 94, 166n3, 200

Krifka, M. 37, 38, 45, 46, 48, 148

Lakoff, G. 8

Langacker, R. W. 219n2

Larson, R. K. 109

Levin, B. 58, 66, 70, 179-80, 184, 185, 186n21, 190

Levin, B. and M. Rappaport Hovav 8, 11, 189, 228

Levinson, L. 14, 31
Lewis, D. 49

lexical entailments $8,10,27,29,216-24$, 230-1

lexical semantic root $21,22-3$

lexical semantics, definition 1

lexicalist event structures $10-11,19-21,90-5$, $110 \mathrm{n} 3$

lexicalization $91-2$

Lexicalization Constraint 192-4

lexicalized conventional associations 222-4

linking rules $6-8,11$

liquefy 222-3

location 6,127

Logical Form (LF) 14

manner entailments $172-8$

manner verbs $96-8,160,167,168,170-8$

Manner/Result Complementarity 27-8, 29-30, 53, 159-206, 209-11, 213-34

manner+result verbs $178-91,195-210$

Marantz, A. 22, 25n11, 201-2

Martin, F. and F. Schäfer 52, 53

maximal endpoint adjectives 42-3, 47

maximalization 38n16

McCawley, J. 1, 113

Megerdoomian, K. 77

melt 222-3

mereological complexity 54, 142n 21

minimal endpoint adjectives $41-2,43$ n24

modality 53

modifiers 68-9

adverbial 83-9, 93, 103

sublexical 16, 24, 89, 93, 95

temporal 18-19, 113

morphological predictions of Bifurcation 74-6, 80

motion scales 127

motion verbs $146,148,165-6$

Movement Relation 38, 39

Müller, A. and L. Sanchez-Mendes 143

murder verbs $179,183-4$

Myler, N. 98

Nedjalkov, V. P. and S. J. Jaxontov 67 nominative/accusative languages 2 non-lexicalist event structures $81-90,94-5$ non-scalar change $172-3$

O’odham 78

object deletion 168-9, 173

objects, differentiation from subjects $2-3$

Oehrle, R. T. 121

offer (French and German) 52

open scale adjectives $41-2$ 
Optimality Theory (OT) 148-9

outprefixation 168-9

participles as adnominal modifiers $64 \mathrm{n} 8$

path scales 127

patient/theme role $6-7,11,13$

PC roots see Property Concept roots

Pedersen, W. A. 88

performativity 141

Pesetsky, D. 121

Peterson, D. A. 155

Phonetic Form (PF) 14

phrasal idioms 25

Pima 78

Pinkal, M. 45

Pinker, S. 21, 110n3, 111, 115

poison verbs 179-80

polysemy 25

positive standard $41-2,44,45-8$

possession 115-17, 119-21

caused 34, 107-14, 120, 187-9

dative alternation $149-50$

scales 127

true possession roots $130-5$

possible and impossible verbs 226-31

possible verb classes $231-3$

predicate argument structure 7

presuppositions $16 \mathrm{n} 9$

Primus, B. 217

production theories of causation 48, 49-50

PROG operator 126

Property Concept (PC) roots 58, 59, 61, 65, 73-4, 77, 78-80, 81, 101, 215

prospective possession $120,121-2$

Pross, T. 90n 23

Pylkkänen, L. 32, 133n18, 154

Quantifier Raising 14-15

Ramchand, G. C. $22-3,32,102,111$

Rappaport Hovav, M. 84, 96, 111, 168, 169-70, $173,178 \mathrm{n} 15,222$

Rappaport Hovav, M. and B. Levin 10, 22, 26-7, $28,96,102 \mathrm{n} 31,110,114,119-20,121$, 159-62, 165, 167, 168, 170n8, 172, 177, $179,188-9,192-4,205$

re-verbs 103,202

reduplication 143

release roots $135-8,139-40$

repetitive readings $16,86-8,94-5,204$

restitutive attachment 199-200, 202

restitutive readings $16,19,86-8,94-5,101$, $153-4,202,205,222$

restricted resultatives $169-72$ result entailments $165-72$

result roots $77-8,79-80,81,83,101,102-3$

manner entailing 96-8

result states $60-1,68,74,78$

result verbs $160,170-2,173-4,176$

resultatives, restricted $169-72$

results, denial $166-7$

ricochet 146

root classes $130-42$

root-determined argument realization 157

root durativity $142-8,157$

root lexical entailments 218, 220, 221

root meaning 23-5

constraints $224-6$

outline of the study 33-5

previous studies $31-3$

root selection $102-3$

root sensitivity $114-17,121,137,157,219$

root sensitivity hypothesis 110

root telicity 124,157

roots

Bifurcation Thesis 26-7, 33-4

compositional analysis $124-30$

in ditransitive verbs $107-11$

flat class and crack class 33

Manner/Result Complementarity 27-8, 29-30

semantic classes $31-2$

vs. templates $28-31$

in change-of-state verbs 104

in ditransitive verbs 155-7

in manner+result verbs $209-10$

typology 30, 227-31

Ross, J. R. 113

run 9,10

scalar change $38 \mathrm{n} 17,41-8,165-6,168-70,177$

scales (of possession or motion) 127

Schwarzschild, R. and K. Wilkinson 45

scrub 168-9

selectional restrictions 173-4

semantic drift 72

semantic predictions of Bifurcation 62-7

Snyder, K. M. 121

Spathas, G. 88-9

Spell-Out 90, 93

'spooky action at a distance' 50

stative participles $82 \mathrm{n} 18$

stativity, temporal $69-71,73,81 \mathrm{n} 17$

Strictly Incremental Relation 37, 39

subjects, differentiation from objects 2-3

sublexical modifiers 16, 24, 89, 93, 95

superlatives 42,43

surface contact verbs $3-4,7,167$ 
Talmy, L. 53n27, 175-6

Tatevosov, S. 51, 53

telicity $36-40,43,137-8$

of ditransitive verbs $122-4$

template lexical entailments $217-18,220$

templates

compositional analysis $13-22,35-55,124-30$

in ditransitive verbs $107-11$

possible verb classes $231-3$

vs. roots $28-31$

in change-of-state verbs 104

in ditransitive verbs $155-7$

in manner+result verbs 209-10

templatic heads 24

temporal modifiers $18-19,113$

temporal stativity $69-71,73,81 \mathrm{n} 17$

Tenny, C. 111

Tham, S. W. 113,149

thematic role hierarchies 7,8

thematic roles 6-8

theme/patient role $6-7,11,13$

throw-type verbs 187-9

to constructions 117-21, 136-7, 151, 157,203

Tongan 78

transfer of possession by motion 134

transformational syntax 14

transitivity 3 true possession roots $130-5$

truth conditions, scalar change $45-8$

Ulwa 23, 76, 78

Uniform Theta-Role Assignment Hypothesis 2

Unique Path Constraint 226

Universal Alignment Hypothesis 2

universal principles, of thematic roles 6-7

Van Valin, R. D. Jr. and R. J. LaPolla 110n3

Van Valin, R. D. Jr. and D. P. Wilkins 14n8, 174

verb meaning $1-5$

event structural approach $6,8-25$

thematic roles 6-8

verb sensitivity hypothesis 110

verbs

arguments 2

classes 3-5, 231-3

deadjectival $43 \mathrm{n} 24$

von Stechow, A. 94, 103

Wolff, P. 175, 176n13

Wolff, P. and G. Song 175, 176n13

Wolff, P. et al. 175, 176n13-14

Wood, J. and A. Marantz 98

word order 2-3, 7

Zucchi, S. and M. White 38n16 


\section{OXFORD STUDIES IN THEORETICAL LINGUISTICS}

\section{PUB L ISHE D}

1 The Syntax of Silence

Sluicing, Islands, and the Theory of Ellipsis by Jason Merchant

2 Questions and Answers in Embedded Contexts

by Utpal Lahiri

3 Phonetics, Phonology, and Cognition edited by Jacques Durand and Bernard Laks

4 At the Syntax-Pragmatics Interface

Concept Formation and Verbal

Underspecification in Dynamic Syntax

by Lutz Marten

5 The Unaccusativity Puzzle

Explorations of the Syntax-Lexicon Interface edited by Artemis Alexiadou, Elena

Anagnostopoulou, and Martin Everaert

6 Beyond Morphology

Interface Conditions on Word Formation

by Peter Ackema and Ad Neeleman

7 The Logic of Conventional Implicatures

by Christopher Potts

8 Paradigms of Phonological Theory edited by Laura Downing, T. Alan Hall, and

Renate Raffelsiefen

9 The Verbal Complex in Romance

by Paola Monachesi

10 The Syntax of Aspect

Deriving Thematic and Aspectual Interpretation Edited by Nomi Erteschik-Shir and Tova

Rapoport

11 Aspects of the Theory of Clitics

by Stephen Anderson

12 Canonical Forms in Prosodic Morphology

by Laura J. Downing

13 Aspect and Reference Time

by Olga Borik

14 Direct Compositionality

edited by Chris Barker and Pauline Jacobson

15 A Natural History of Infixation

by Alan C. L. Yu

16 Phi-Theory

Phi-Features Across Interfaces and Modules edited by Daniel Harbour, David Adger, and Susana Béjar

\section{French Dislocation}

Interpretation, Syntax, Acquisition

by Cécile De Cat

18 Inflectional Identity

edited by Asaf Bachrach and Andrew Nevins

19 Lexical Plurals

by Paolo Acquaviva
20 Adjectives and Adverbs

Syntax, Semantics, and Discourse

Edited by Louise McNally and Christopher

Kennedy

21 InterPhases

Phase-Theoretic Investigations of Linguistic

Interfaces

edited by Kleanthes Grohmann

22 Negation in Gapping

by Sophie Repp

23 A Derivational Syntax for Information

Structure

by Luis López

24 Quantification, Definiteness, and

Nominalization

edited by Anastasia Giannakidou and Monika

Rathert

25 The Syntax of Sentential Stress

by Arsalan Kahnemuyipour

26 Tense, Aspect, and Indexicality

by James Higginbotham

27 Lexical Semantics, Syntax, and Event

Structure

edited by Malka Rappaport Hovav, Edit Doron, and Ivy Sichel

28 About the Speaker

Towards a Syntax of Indexicality

by Alessandra Giorgi

29 The Sound Patterns of Syntax

edited by Nomi Erteschik-Shir and Lisa

Rochman

30 The Complementizer Phase

edited by Phoevos Panagiotidis

31 Interfaces in Linguistics

New Research Perspectives

edited by Raffaella Folli and Christiane Ulbrich

32 Negative Indefinites

by Doris Penka

33 Events, Phrases, and Questions

by Robert Truswell

34 Dissolving Binding Theory

by Johan Rooryck and Guido Vanden Wyngaerd

35 The Logic of Pronominal Resumption

by Ash Asudeh

36 Modals and Conditionals

by Angelika Kratzer

37 The Theta System

Argument Structure at the Interface

edited by Martin Everaert, Marijana Marelj, and Tal Siloni

38 Sluicing

Cross-Linguistic Perspectives

edited by Jason Merchant and Andrew Simpson 
39 Telicity, Change, and State

A Cross-Categorial View of Event Structure edited by Violeta Demonte and Louise McNally

40 Ways of Structure Building

edited by Myriam Uribe-Etxebarria and Vidal Valmala

41 The Morphology and Phonology of Exponence

edited by Jochen Trommer

42 Count and Mass Across Languages edited by Diane Massam

43 Genericity

edited by Alda Mari, Claire Beyssade, and Fabio Del Prete

44 Strategies of Quantification

edited by Kook-Hee Gil, Steve Harlow, and George Tsoulas

45 Nonverbal Predication

Copular Sentences at the Syntax-Semantics

Interface

by Isabelle Roy

46 Diagnosing Syntax

edited by Lisa Lai-Shen Cheng and Norbert

Corver

47 Pseudogapping and Ellipsis

by Kirsten Gengel

48 Syntax and its Limits

edited by Raffaella Folli, Christina Sevdali, and Robert Truswell

49 Phrase Structure and Argument Structure A Case Study of the Syntax-Semantics Interface by Terje Lohndal

50 Edges in Syntax

Scrambling and Cyclic Linearization by Heejeong Ko

51 The Syntax of Roots and the Roots of Syntax edited by Artemis Alexiadou, Hagit Borer, and Florian Schäfer

52 Causation in Grammatical Structures edited by Bridget Copley and Fabienne Martin

53 Continuations and Natural Language by Chris Barker and Chung-chieh Shan

54 The Semantics of Evaluativity by Jessica Rett

55 External Arguments in Transitivity

Alternations

A Layering Approach

by Artemis Alexiadou, Elena Anagnostopoulou, and Florian Schäfer

56 Control and Restructuring

by Thomas Grano

57 The Interaction of Focus, Givenness, and Prosody

A Study of Italian Clause Structure

by Vieri Samek-Lodovici
58 The Morphosyntax of Gender

by Ruth Kramer

59 The Morphosyntax of Imperatives

by Daniela Isac

60 Sentence and Discourse

edited by Jacqueline Guéron

61 Optimality-Theoretic Syntax, Semantics, and Pragmatics

From Uni- to Bidirectional Optimization edited by Géraldine Legendre, Michael T.

Putnam, Henriëtte de Swart, and Erin

Zaroukian

62 The Morphosyntax of Transitions

A Case Study in Latin and Other Languages

by Víctor Acedo-Matellán

63 Modality Across Syntactic Categories

edited by Ana Arregui, María Luisa Rivero, and Andrés Salanova

64 The Verbal Domain

edited by Roberta D'Alessandro, Irene Franco, and Ángel J. Gallego

65 Concealed Questions

by Ilaria Frana

66 Parts of a Whole

Distributivity as a Bridge between Aspect and

Measurement

by Lucas Champollion

67 Semantics and Morphosyntactic Variation Qualities and the Grammar of Property

Concepts

by Itamar Francez and Andrew

Koontz-Garboden

68 The Structure of Words at the Interfaces

edited by Heather Newell, Máire Noonan, Glyne Piggott, and Lisa deMenaTravis

69 Pragmatic Aspects of Scalar Modifiers

The Semantics-Pragmatics Interface

by Osamu Sawada

70 Encoding Events

Functional Structure and Variation

by Xuhui $\mathrm{Hu}$

71 Gender and Noun Classification

edited by Éric Mathieu, Myriam Dali, and Gita

Zareikar

72 The Grammar of Expressivity

by Daniel Gutzmann

73 The Grammar of Copulas Across Language edited by María J. Arche, Antonio Fábregas, and Rafael Marín

74 The Roots of Verbal Meaning

by John Beavers and Andrew Koontz-Garboden

Published in association with the series

The Oxford Handbook of Linguistic Interfaces edited by Gillian Ramchand and Charles Reiss 


\section{In preparation}

Phi Syntax

A Theory of Agreement

by Susana Béjar

Stratal Optimality Theory

by Ricardo Bermúdez Otero

Contrast and Representation in Syntax

edited by Bronwyn M. Bjorkman and

Daniel C. Hall

Phonology in Phonetics

by Abigail Cohn

Quantity Superlatives and Proportional

Quantification

by Carmen Dobrovie-Sorin and Ion Giurgea
Generality and Exception

by Ivan Garcia-Alvarez

The Derivational Timing of Ellipsis edited by Güliz Günes and Anikó Lipták

Computing Optimality

by Jason Riggle

Gradience in Split Intransitivity

by Antonella Sorace

Syntactic Reconstruction in Minimalism

by Dominique Sportiche

The Syntax of Perspectival Anaphora by Sandhya Sundaresan

Negation and Negative Dependencies by Hedde Zeijlstra 











